\title{
QUANTITATIVE ANALYSIS OF ANGIOGENESIS IN MICROFLUIDIC DEVICES
}

\section{WANG MENGMENG}

School of Electrical \& Electronic Engineering

A thesis submitted to the Nanyang Technological University in fulfillment of the requirements for the degree of

Doctor of Philosophy 


\section{Statement of Originality}

I hereby certify that the work embodied in this thesis is the result of original research and has not been submitted for a higher degree to any other University or Institution.

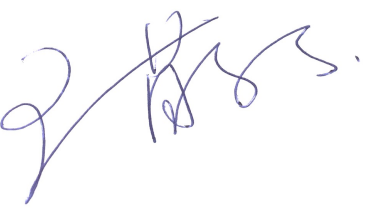

Date

WANG MENGMENG 


\section{Acknowledgements}

First of all, I would like to express my special and great appreciation to my supervisor, Prof. Justin Dauwels, for his invaluable guidance and continuous support throughout my Ph.D study. His encouragement and praise have brought me through the difficulties and harsh times in my Ph.D journey. This thesis would never have been possible without his brilliant ideas and extraordinary drive for research. He is not only my $\mathrm{PhD}$ supervisor, but also my life mentor.

I wish to express my deep gratitude to my co-supervisor, Prof. H. Harry Asada from Massachusetts Institute of Technology, for involving me in this interdisciplinary research project. His perpetual energy and enthusiasm in research extremely motivated me in my studies. He is so patient and always willing to help whenever I have questions.

My sincere thanks to Dr. Lee-Ling Sharon Ong from Singapore-MIT Alliance for Research and Technology (SMART) Centre, for her kindly help and instruction in my research. I also want to thank my wonderful fellow lab mates, who have made my Ph.D journey more meaningful and enjoyable.

Last but not least, I would like to express my sincere thanks to my family, my husband Dr. Du Xinxin, my father Mr. Wang Bingjun, and my mother Ms. Shi Yuling, for their constant love, encouragement and support. Without them as my backup, I would fail half way. 


\section{Abstract}

Angiogenesis is the growth of new blood vessels from existing vessels. It is a critical process in fetal development, wound healing, and growth and development. However, it also plays an important role in cancer growth and spread, which is one of the leading causes of deaths worldwide. Better understanding of angiogenic mechanism is required to develop effective antiangiogenic therapies for cancer treatment.

During angiogenesis, endothelial cells (ECs) migrate in a coordinated manner by specializing into two distinct phenotypes: tip cells and stalk cells. Tip cells extend dynamic filopodia to sense and respond to angiogenic stimuli, and stalk cells trail behind tip cells to form solid vessels. Both cell phenotypes can dynamically switch positions and functions during the sprouting process. The inter-transition of the cell phenotypes plays an important role for ECs sprouting out from monolayer, extending and creating new branches, as well as reconnecting in a later stage.

Automated image analysis tools to analyze experimental microscopy images are useful for a wide range of biomedical investigations, since they can provide quantitative understanding of the biological processes for biologists and generate numerical data to build computational models. In this thesis, we focus on ECs migration and angiogenic vascular formation for a better understanding of angiogenesis. Therefore, our objectives include culturing angiogenic vessels with branching in $3 \mathrm{D}$ in vitro environments, developing automated image analysis systems to track multiple ECs migration and to track the angiogenic vessel formation. In the following, we will 
discuss each objective in detail.

Firstly, we conduct angiogenic experiments in the 3D microfluidic devices (MFDs), which mimic the in vivo system. By providing tumor angiogenic growth factors (TAFs) such as Vascular Endothelial Growth Factor (VEGF) and Sphingosine-1phosphate (S1P) in the 3D MFDs, angiogenic vessels with branching are formed. The sprouting processes are observed with a phase contrast microscope daily and with a confocal microscope at the end time. To investigate the influence of S1P on angiogenic vessel morphologies, we consider three different S1P conditions. The experimental observations suggest that the positive S1P gradient increases the average length of the angiogenic vessels.

Secondly, we develop an automated multi-cell tracking system (AMCTS) to track the migrating ECs within the angiogenic vessels from the time-lapse phase contrast images. The proposed system consists of preprocessing to eliminate the non-gel region and obtain binary angiogenic vessel shapes, cell detection to find and label the EC candidates, and multiple hypothesis Kalman filtering to associate and track the detected EC candidates over the image sequences. Biological knowledge is incorporated when estimating the track probability during cell association. Numerical results indicate that the proposed system is able to track the cell migration trajectories accurately. Cell lineage plots, showing the history of the cell proliferation and cell migration into the gel, with timestamps of when it is in-focus and out-of-focus, are also generated automatically from this system.

Thirdly, we propose an automated vessel formation tracking system (AVFTS) to track the vessel formation and extract quantitative vessel information from the timelapse phase contrast images. The proposed system consists of preprocessing, skeletonization, and branch tracking. After obtaining the binary vessel shapes through preprocessing, we apply a distance transform (DT) and an augmented fast marching method (AFMM) to extract the vessel skeletons, which are a group of segments connected by nodes. Next, we train a support vector machine (SVM) classifier to 
distinguish and remove the specious segments which are not part of the vessels. Lastly, we identify the route for each branch by joining the linked skeleton segments and associate the branches over image sequences by the Hungarian method. This system is applicable to distinguish tip and stalk cells based on their relative cell positions within a branch. Moreover, it also helps biologists to investigate the influence of different angiogenic factors by automatically extracting quantitative vessel information including vessel length, vessel width, and the number of branches from experimental microscopy images. Specifically, we quantify the experimental results under different S1P conditions and find that the positive S1P gradient increases ECs migration and vessel elongation, and leads to higher possibilities for branching to occur.

In conclusion, we culture angiogenic vessels over long period of time in 3D MFDs and develop two automated image analysis systems to quantitatively analyze these angiogenic vessels. These systems would benefit biologists by providing quantitative and accurate comparison of the influence of different growth factors on angiogenic vessel morphologies. They also provide numerical data to build computational models for angiogenesis prediction. 


\title{
List of Abbreviations
}

\author{
AFMM augmented fast marching method \\ AMCTS automated multi-cell tracking system \\ AVFTS automated vessel formation tracking system \\ DT distance transform \\ EC endothelial cell \\ ECM extracellular matrix \\ EGM endothelial cell growth media \\ FMM fast marching method \\ HMVECs human microvascular endothelial cells \\ HT hough transform \\ HUVECs human umbilical vein endothelial cells \\ IMM interacting multiple model \\ MAT medial axis transform \\ MFD microfluidic device \\ MHT multiple hypothesis tracking \\ MMP matrix metalloproteinase \\ PCA principal component analysis \\ PDMS polydimethylsiloxane \\ PLSR partial least square regression \\ S1P sphingosine-1-phosphate \\ SURF speeded up robust feature \\ SVM support vector machine
}


TAF tumor angiogenic growth factor

TPM transition probability matrix

VEGF vascular endothelial growth factor 


\section{Contents}

Abstract $\quad$ vi

List of Abbreviations $\quad$ ix

List of Contents $\quad$ xi

List of Figures xix

List of Tables $\quad$ xxi

1 Introduction $\quad 1$

1.1 Biological Background of Angiogenesis . . . . . . . . . . . . . . 1

1.2 Motivations and Objectives f . . . . . . . . . . . . . 7

1.3 Thesis Organization and Overview of Contributions . . . . . . . . . 10

2 Experimental Materials and Methods 
2.1 Introduction of MFDs . . . . . . . . . . . . . . . . . 13

2.2 MFDs Fabrication . . . . . . . . . . . . . . . . . 15

2.3 Experimental Conditions . . . . . . . . . . . . . . . . 17

2.4 Angiogenic Experimental Observations . . . . . . . . . . . . . . . 21

3 Automatic Tracking of Endothelial Cells in Angiogenic Vessels 25

3.1 Introduction and Literature Review . . . . . . . . . . . . . . . 26

3.2 Automated Multi-Cell Tracking System . . . . . . . . . . . . . . . . 28

3.2.1 Preprocessing . . . . . . . . . . . . . . . . . . . . 29

3.2 .2 Cell Detection . . . . . . . . . . . . . . . . . 38

3.2.3 Multiple Hypothesis Kalman Filtering . . . . . . . . . . . . . 42

3.3 Results and Discussion . . . . . . . . . . . . . . . . . . . . 49

3.3.1 Preprocessing Results . . . . . . . . . . . . . 50

3.3.2 Cell Detection Results . . . . . . . . . . . . 50

3.3.3 Tracking Results . . . . . . . . . . . . . . . . . 57

3.4 Conclusion . . . . . . . . . . . . . . . . . . . . . 62

4 Automatic Tracking of Angiogenic Vessel Formation 65

4.1 Introduction and literature review . . . . . . . . . . . . 66

4.2 Automated Vessel Formation Tracking System . . . . . . . . . . . . . 68

4.2 .1 Skeletonization . . . . . . . . . . . . . . . . 69 
4.2 .2 Branch Tracking . . . . . . . . . . . . . . . . 81

4.3 Results and Discussion . . . . . . . . . . . . . . . . . . . 93

4.3.1 Tracking of Vessel Formation . . . . . . . . . . . . . . . 93

4.3 .2 Application of the AVFTS . . . . . . . . . . . . . 93

4.4 Conclusion . . . . . . . . . . . . . . . . . . . . 104

5 Conclusions and Future Work

5.1 Summary of Contributions . . . . . . . . . . . . . . . 107

5.2 Recommendations for Future Work . . . . . . . . . . . . . . . 110

5.2.1 Multi-cell Tracking by Interacting Multiple Model Motion Filter110

5.2.2 Computation Models for Branching and Anastomosis in Angiogenesis . . . . . . . . . . . . . . . . . . 112 


\section{List of Figures}

1.1 Role of angiogenesis in tumor growth [1]: (a) the avascular primary tumor with small size; (b) tumor cells produce angiogenic growth factors to induce angiogenesis; and (c) large vascularized tumor. . . . 2

1.2 Angiogenic sprouting process: (a) activation of ECs by TAFs; (b) tip cell selection via VEGF/Notch signaling pathways; (c) vessel elongation; (d) branching; (e) vessels undergoing anastomosis; and (f) vessels after anastomosis. . . . . . . . . . . . . . . . . . 6

2.1 Examples of conventional in vivo and in vitro models for angiogenesis studies. . . . . . . . . . . . . . . . . . . . . 14

2.2 "High throughput" MFD [2]: (a) external appearance; (b) internal appearance with 37 sprouting regions; and (c) individual sprouting region. . . . . . . . . . . . . . . . 15

2.3 Comparison of the migration distances of EC nuclei in $x y$-plane and $z$-direction in the MFDs. . . . . . . . . . . . . . . 16

2.4 Examples of angiogenic vessels (without S1P) in phase contrast images for 5 consecutive days (from day 4 to day 8 after cell seeding). . 19

2.5 Breakage of tip cell under high S1P concentration. . . . . . . . . . 20 
2.6 Example of angiogenic vessels in phase contrast image under 4x magnification.

2.7 Example of angiogenic vessels in a sprouting region by stitching the overlapping phase contrast images under 20x magnification: corresponds to the green box in Fig. 2.6(a). . . . . . . . . . . . . . . 23

2.8 Example of angiogenic vessels in the same sprouting region as in Fig. 2.7 in the end-point confocal image: cell nuclei are in blue and actin is in green. . . . . . . . . . . . . . 23

2.9 Section view of angiogenic vessels in MFDs in the confocal image. . . 24

3.1 Examples of cells: (a) on a 2D surface and (b) within 3D vessels (red arrows point to cells). . . . . . . . . . . . . . . . . 27

3.2 Diagram of the proposed automated tracking system: preprocessing to remove the non-gel region and segment the binary vessel shapes for all the aligned experimental images; cell detection to label all EC candidates; and multiple hypothesis Kalman filtering to associate the detected EC candidates. . . . . . . . . . . . . . . . . . . . 28

3.3 Examples of overlapping phase contrast images for angiogenic vessels in a same sprouting region over 4 consecutive days (day 9 to day 12). 30

3.4 Stitching results for the examples in Fig. 3.3 showing the entire angiogenic vessels in a same sprouting region over 4 consecutive days

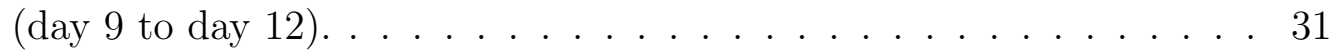

3.5 Features for image registration: line 1 to line 4 are represented by the Hough Transform, and the red points are the correspondence points. . 32

3.6 Hough Transform algorithm. . . . . . . . . . . . . . . . . . . 33 
3.7 Image registration results for the examples in Fig. 3.3 showing the angiogenic vessels in a same sprouting region over 4 consecutive days (day 9 to day 12). . . . . . . . . . . . . . . . . 34

3.8 Example of the cell-gel interface in an experimental image. . . . . . . 35

3.9 Images after masking out the non-gel region for the examples in Fig. 3.3: angiogenic vessels in a same sprouting region over 4 consecutive days (day 9 to day 12 ) . . . . . . . . . . . . . 36

3.10 Segmented binary shapes for the examples in Fig. 3.3: angiogenic vessels in the same sprouting region over 4 consecutive days (day 9

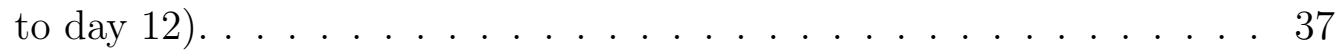

3.11 Template matching flowchart: The binary classifier is trained offline by means of discriminative features. After training, the classifier is applied to a sliding window that runs through the entire image, and distinguishes cells from background in each window. . . . . . . . . . 39

3.12 Examples of cell and non-cell templates from phase contrast images for offline training. . . . . . . . . . . . . . . . . . . . . 39

3.13 Diagram of partial least square regression. . . . . . . . . . . . . . . 40

3.14 Percent of variance explained v.s. the number of PLS components. . . 42

3.15 Backward Kalman filter with validation gating for cell association: blue and red ellipses are the validation gate for track 1 and 2 respectively; track 1 is associated with observation 1 and track 2 is associated with observation 2 in this plot. . . . . . . . . . . . 45 
3.16 Flowchart of multiple hypothesis Kalman filtering. Cell state, system covariance, and probabilities of all the tracks are recursively updated over time, corresponding to multiple hypotheses. The cell associations are obtained by selecting the most probable hypotheses. . . . . . . . . 47

3.17 Multiple hypothesis Kalman filtering for one cell in two successive time steps. . . . . . . . . . . . . . . . . 48

3.18 Illustration of preprocessing results: (a) and (e) are the end-point phase contrast images; (b) and (f) are their corresponding confocal images; (c) and (g) show the co-registered confocal and phase contrast images (the nuclei color is changed from blue to green just for clearer visualization); (d) and (h) are the binary vessel shapes from vessel segmentation. . . . . . . . . . . . . . . 52

3.19 Examples of cell detection results for different approaches: (a) and (d) from the proposed cell detection approach using PLSR; (b) and (e) from an SVM classifier based on original high-dimensional template data; (c) and (f) from an SVM classifier based on the dimensionreduced features after PCA. Red stars $(*)$ indicate the detected nuclei locations. Examples of FN and FP are labeled in (d). . . . . . . . . 55

3.20 Cell tracking example I. (a) and (b): Backward Kalman filter with MHT; (c) and (d): backward Kalman filter with validation gating. Each image shows the outcomes of the tracking algorithms for two consecutive frames, where the phase contrast image is shown in the background. . . . . . . . . . . . . . . . . 58 
3.21 Cell tracking example II. (a) and (b): Backward Kalman filter with MHT; (c) and (d): backward Kalman filter with validation gating. Each image shows the outcomes of the tracking algorithms for two consecutive frames, where the phase contrast image is shown in the background.. . . . . . . . . . . . . . . . . . 59

3.22 Cell lineage plots. The blue lines represent the cells that migrate within the 3D gel; the red stars represent the incoming cells from the cell-gel interface, whereas the green stars indicate the cells migrating out-of-focus. . . . . . . . . . . . . . . .

4.1 Diagram of the automated vessel formation system: preprocessing to generate binary vessel shape, skeletonization to obtain the connected skeleton segments with branch nodes and end notes, and branch tracking to identify the routes for the main branches and associate the

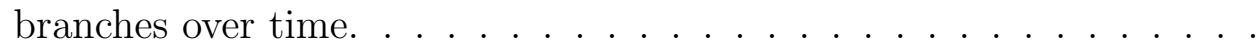

4.2 Example of (a) skeleton segments and nodes and (b) one segment: branch nodes (yellow), end nodes (green), segments (blue lines), and vessel radii (red circles).

4.3 Demonstration of AFMM algorithm to find the skeleton of a simple rectangular shape.

4.4 An example of skeletonization: (a) binary vessel shape; (b) boundary of the binary vessel; (c) skeletons from thinning; (d) skeletons from DT and AFMM; (e) skeleton segments with nodes; (f) skeleton segments with radii in the original image; (g) an example of a filopodia wrongly labeled as skeleton; and (h) skeleton segments with radii after SVM classification. . . . . . . . . . . . . . . . . . 74

4.5 Diagram of support vector machine. . . . . . . . . . . . 75 
4.6 Examples of training templates for the binary SVM classifier: (a) and (b) are vessels from confocal image and phase contrast image respectively; (c) shows skeleton segments and nodes from DT and AFMM; (d) are positive templates; and (e) are negative templates. . 79

4.7 Base nodes determination for the two different scenarios: (a) and (b) are the skeletons with radii in the experimental images for the two scenarios respectively; (c) and (d) are their corresponding generalized schematic diagrams; (e) and (f) indicate the based nodes in red. . . . 82

4.8 Diagram to illustrate the nodes and segments for identifying the route for each branch. . . . . . . . . . . . . . . . . . . . . 84

4.9 Identified branch routes for the example in Fig. 4.7(a): each branch of the vessels is plotted in (b) to (d), where the blue lines are the continuous skeletons and the red circles are the branch radii at selected points in the skeletons. . . . . . . . . . . . . . . . 86

4.10 Identified branch routes for the example in Fig. 4.7(b): each branch of the vessels is plotted in (b) to (d), where the blue lines are the continuous skeletons and the red circles are the branch radii at selected points in the skeletons. . . . . . . . . . . . . . 87

4.11 Skeletons of the angiogenic vessels over two consecutive days: the vessels in the red rectangular box and in the yellow box are originally from two different tip cells that migrated from the monolayer. . . . . 88

4.12 Skeletons and nodes for angiogenic vessels of two consecutive days. . . 92

4.13 Example 1 for branch tracking of angiogenic vessels within a sprouting region from day 5 to day 9 .

4.14 Example 2 for branch tracking of angiogenic vessels within a sprouting region from day 5 to day 9 . 
4.15 Diagram of tip cell identification: (a) tip cell candidates and (b) tip cell selection.

4.16 An example of tip cell identification result. (a) Angiogenic vessels with both cell and branch information: cell locations plotted as green stars, branch skeletons plotted as blue lines, and branch radii plotted as red circles; (b) Tip cells identified based on the distances between the cells and the end nodes only: tip cells plotted in yellow stars; and (c) Tip cells identified with our approach: tip cells plotted in yellow stars. . . . . . . . . . . . . . . . . . . 98

4.17 Angiogenic vessels observed by phase contrast microscopy (at 4x magnification) at day 9 under different S1P conditions: (a) No S1P, (b) Positive S1P gradient, and (c) Negative S1P gradient. . . . . . . . . . 100

4.18 Quantitative comparison of vessel morphologies under different S1P conditions: (a) branch length $(N=24 \sim 36)$; (b) branch radius $(N=24 \sim 36)$; and (c) the number of branches in a sprouting region $(N=12)$. The error bars give the mean and standard deviation. . . . 102

4.19 Change of average branch length over time for different S1P conditions: the error bars give the mean with SEM. . . . . . . . . . . . 104

5.1 Interacting multiple model switching process. . . . . . . . . . . . . 110

5.2 Multiple aspects of investigating a biological process. . . . . . . . . . 112 


\section{List of Tables}

2.1 Different S1P conditions in the angiogenic experiments. . . . . . . . . 21

3.1 Confusion matrix for a binary classifier [3]. . . . . . . . . . . . 53

$3.2 \mathrm{TP}, \mathrm{FP}$, and FN values for different cell detection approaches. . . . . 56

3.3 Classification performance of cell detection (in \%). . . . . . . . 56

3.4 Cell association accuracy for different approaches: I. Nearest-neighborhood association; II. Kalman filter with validation gating; III. the Hungarian method; and IV. Multiple hypothesis Kalman filtering. . . . . . . 60

4.1 Branches for the vessels in Fig. $4.12 \ldots$. . . . . . . . . . . . . . 91

4.2 Examples of the branch formation tracking results. . . . . . . . . . . 92

4.3 Symbols for different $\mathrm{P}$ values ranges. . . . . . . . . . . . . . . . . 101

5.1 Explanation of elements in TPM. . . . . . . . . . . . . . 111 


\section{Chapter 1}

\section{Introduction}

Summary - We present the overview of this thesis in this chapter. Specifically, we start with the biological background of the angiogenesis in Section 1.1 and elaborate on our motivations and objectives in Section 1.2. In Section 1.3, we provide the organization of this thesis and the summary of our contributions.

\subsection{Biological Background of Angiogenesis}

Angiogenesis is the growth of new blood vessels from pre-existing vessels [4]. It is a critical process in fetal development, wound healing, and growth and development [5]. It also plays an important role in cancer growth and spread [6], which is one of the leading causes of deaths worldwide $[7,8,9,10]$. According to the estimates from International Agency for Research on Cancer (IARC), 8.2 million humans died due to cancer in 2012 worldwide and the deaths are estimated to rise to 13.1 million in 2030 [11]. For the past few decades, scientists focus on revealing the angiogenesis process in cancer biology, for the development of clinical therapeutics [12].

Most cancers in the human body form lumps of tissues, namely solid tumors. Initially, solid tumor is avascular and relies on its nearby blood vessels to get oxygen 
and nutrients (see Fig. 1.1(a)). When the tumor reaches a size of $1 \mathrm{~mm}-3 \mathrm{~mm}$, the nutrients from the tumor surface cannot fulfil its growth demand and the oxygen cannot reach the cells at the center of the tumor due to the oxygen diffusion limit [13]. The tumor stops growing unless it becomes vascularized [6]. As a response to this oxygen deprivation (also called hypoxia), the tumor will produce the Tumor Angiogenic Growth Factors (TAFs) to induce nearby blood vessels to grow new capillaries to vascularize the tumor through angiogenesis [14, 15], as shown in Fig. 1.1(b). The new-formed vascular within the tumor will then provide the nutrients and oxygen for tumor survival and growth (see Fig. 1.1(c)). To develop effective antiangiogenenic therapies for cancer treatment, a better understanding of the underlying angiogenic mechanism is required [16, 17].

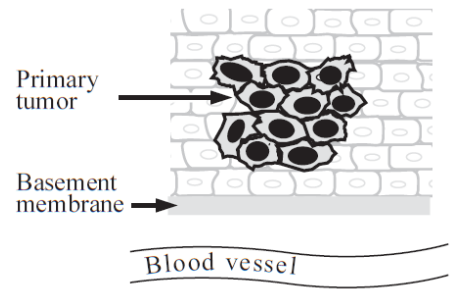

(a) Avascular tumor.

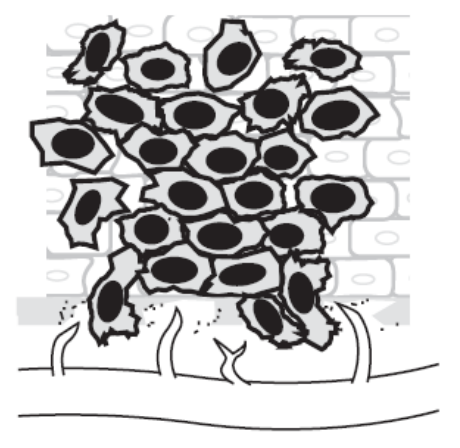

(b) Activation of angiogenesis.

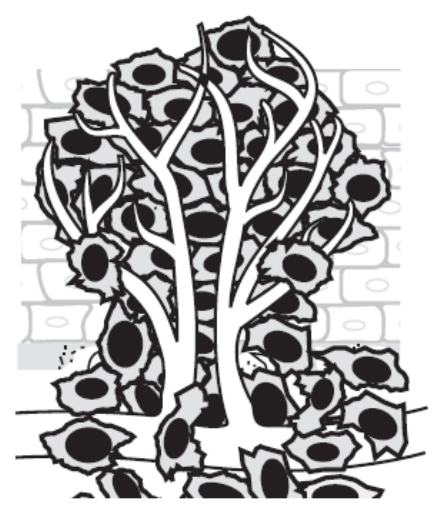

(c) Vascularized tumor.

Figure 1.1: Role of angiogenesis in tumor growth [1]: (a) the avascular primary tumor with small size; (b) tumor cells produce angiogenic growth factors to induce angiogenesis; and (c) large vascularized tumor.

During angiogenesis, endothelial cells (ECs) migrate in a coordinated manner, specializing into two distinct phenotypes: tip cells and stalk cells [18]. Tip cells extend dynamic filopodia to sense and respond to angiogenic stimuli, and stalk cells trail behind tip cells to form solid vessels. Both cell phenotypes can dynamically switch positions and functions during the sprouting process [19, 20]. The inter-transition of the cell phenotypes is critical for proper vascular formation in angiogenesis since it plays an important role for ECs sprouting out from monolayer, extending and creating new branches, as well as anastomosis in a later stage [21, 22, 23]. 
Growth factors play important roles in coordinating multiple cellular interactions during angiogenesis. Numerous factors have been shown to influence the angiogenesis process, such as vascular endothelial growth factors (VEGF), sphingosine-1phosphate (S1P), transforming growth factor beta (TGF- $\beta$ ), basic fibroblast growth factor (bFGF), placental growth factor (PGF), tumor necrosis factors (TNF), angiopoietin 1 (Ang-1) [24], and platelet factor 4 (PF4) [25].

VEGF is one of the major regulators for blood vessel formation and function in angiogenesis $[26,27]$. Existing studies have shown that VEGF gradient induces the growth of new capillaries from existing vessels [28]. Moreover, VEGF also promotes EC proliferation [29, 30], migration [31], and survival [32] in angiogenesis. VEGF receptor expressions are different for tip cells and stalk cells, indicating that VEGF pathway participates in the endothelial phenotypic heterogeneity and is critical for proper vascular network formation [18]. S1P is a biologically active lipid that has been shown to regulate diverse biological functions such as cell growth, migration, and survival [33, 34]. It is also involved in angiogenesis to stimulate cell invasion, lumen formation, and branching morphogenesis [35]. Moreover, S1P has been postulated to cross-communicate with the VEGF pathway in a feed-forward loop promoting the development of blood vessels [36, 37].

In solid tissues, cells are surrounded by extracellular matrix (ECM) which is a collection of extracellular molecules secreted by cells [38, 39]. The ECM not only provides structural support to the surrounding cells, but also regulates multi-cell behaviors (e.g. cell-cell adhesion and cell-cell communication) through biomechanical signals [40, 41]. During angiogenesis, tip cells secrete Matrix Metalloproteinases (MMPs) to degrade the ECM and create conduits for stalk cells migration and proliferation [6]. Studies have shown that both the biochemical properties and mechanical properties (e.g. stiffness) of ECM are critical influencing factors for ECs migration in angiogenesis [42, 43].

As illustrated in Fig. 1.2(a), sprouting angiogenesis starts with the activation of 
ECs when they are exposed to TAFs. ECs will compete for the tip cell positions to limit the number of outgrowing sprouts [19]. Recent studies have shown that the tip cell selection process highly relies on VEGF pathway and notch signaling pathway $[4,15,18,44,45,46,47,48]$. Notch signaling pathway is a short range communication system between two adjacent cells through 4 receptors (Notch 1-4) and 5 ligands (DLL1, DLL3, DLL4, Jagged 1, and Jagged 2) [49].

When one EC (e.g. the green cell in Fig. 1.2(b)) receives VEGF signal by VEGF receptors, a signaling cascade is triggered to increase its VEGF-induced DLL4 expression, which enables this EC to become a tip cell. These DLL4 ligands activate notch signaling in its adjacent cells (e.g. the orange cells in Fig. 1.2(b)) to downregulate their VEGF receptor signaling, and to dampen their VEGF-induced expression of DLL4. As a result, the tip cell receives low notch signaling from its adjacent cells and has high expression of VEGF receptors. This self-reinforcing feedback allows the tip cell (the green cell in Fig. 1.2(b)) to retain its tip position and inhibits its neighboring cells (the orange cells in Fig. 1.2(b)) from becoming tip cells.

Tip cells in the leading positions extend the long dynamic filopodia to sense and respond to the angiogenic stimuli (e.g. the VEGF gradient), and lead the way [44]. Stalk cells trail behind the tip cells along the conduits, form solid sprouts or lumen vessels, and maintain connectivity with parent vessels. Fig. 1.2(c) shows the formed vessels, where tip cell and stalk cells are as labeled. The green color in tip cell and orange color in selected stalk cells are to demonstrate the VEGF/Notching signaling effect.

The balance between tip and stalk cell phenotypes must be tightly controlled to ensure a correct development of the vasculature [50]. The transient modus of notch signaling pathway promotes the dynamic inter-transition of tip and stalk cells, leading to branching and anastomosis [21]. After the sprout extends for some distance, a stalk cell, which is typically not adjacent to a tip cell, occasionally converts to a tip cell. This is where a new branch forms, referred to branching. The newly 


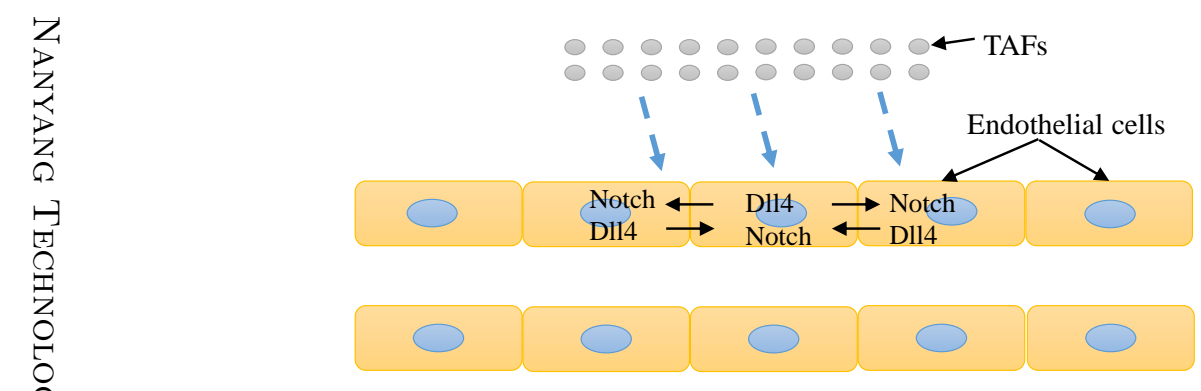

(a) Activation of ECs by TAFs.

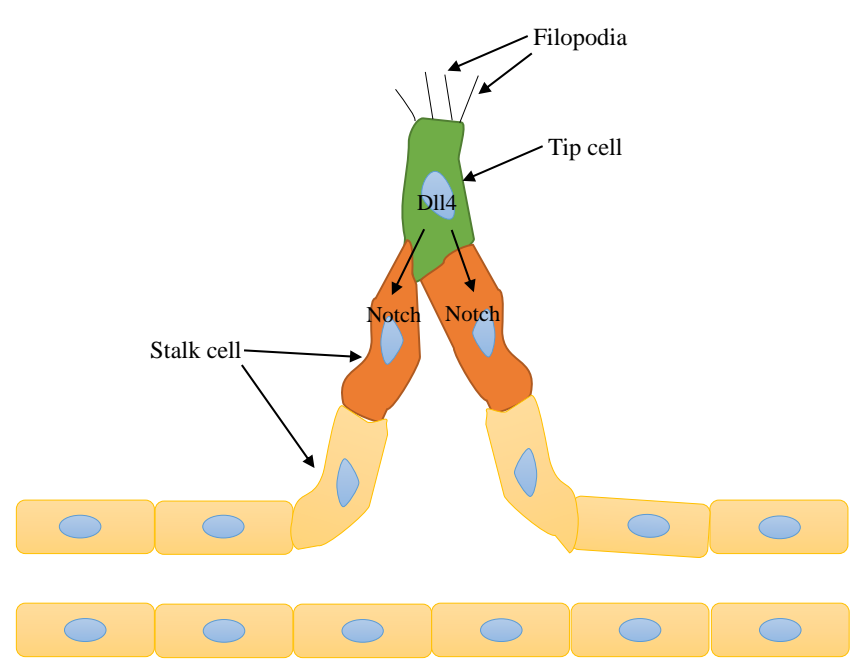

(c) Vessel formation.

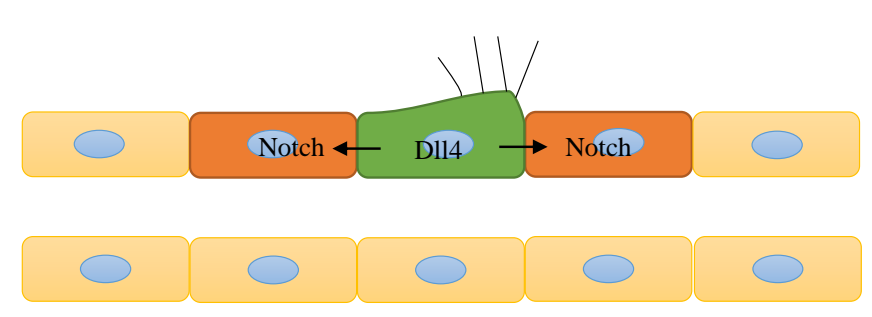

(b) Tip cell selection.

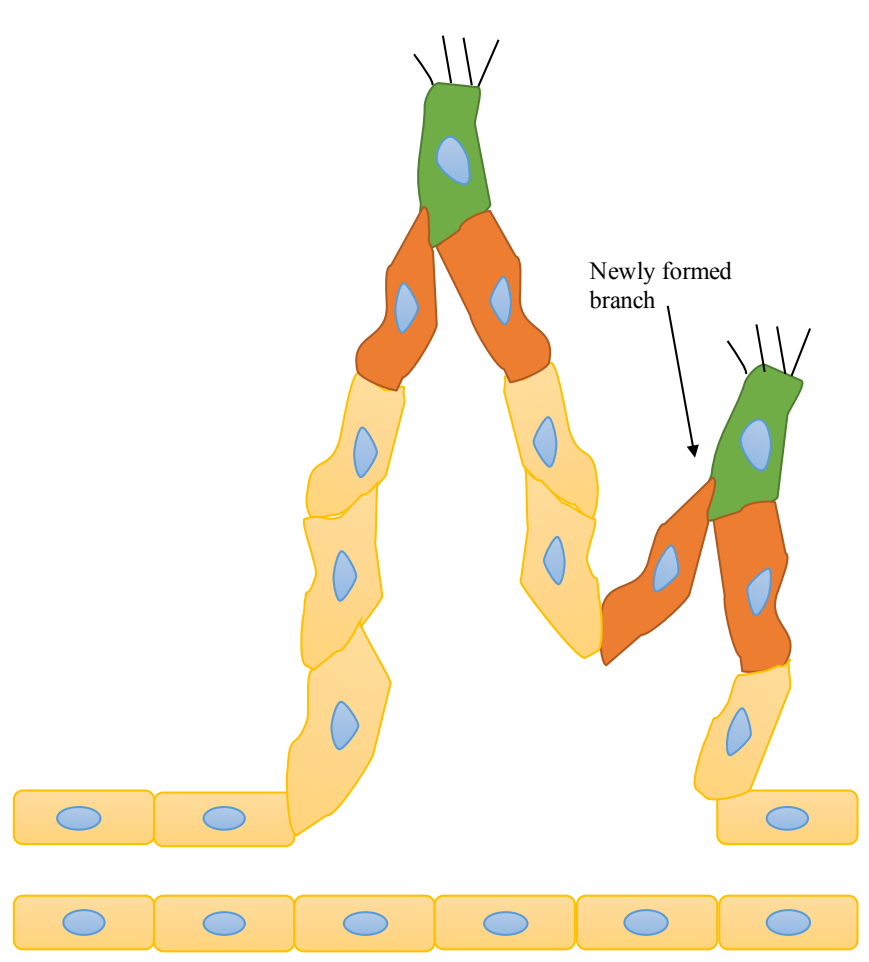

(d) Branching. 


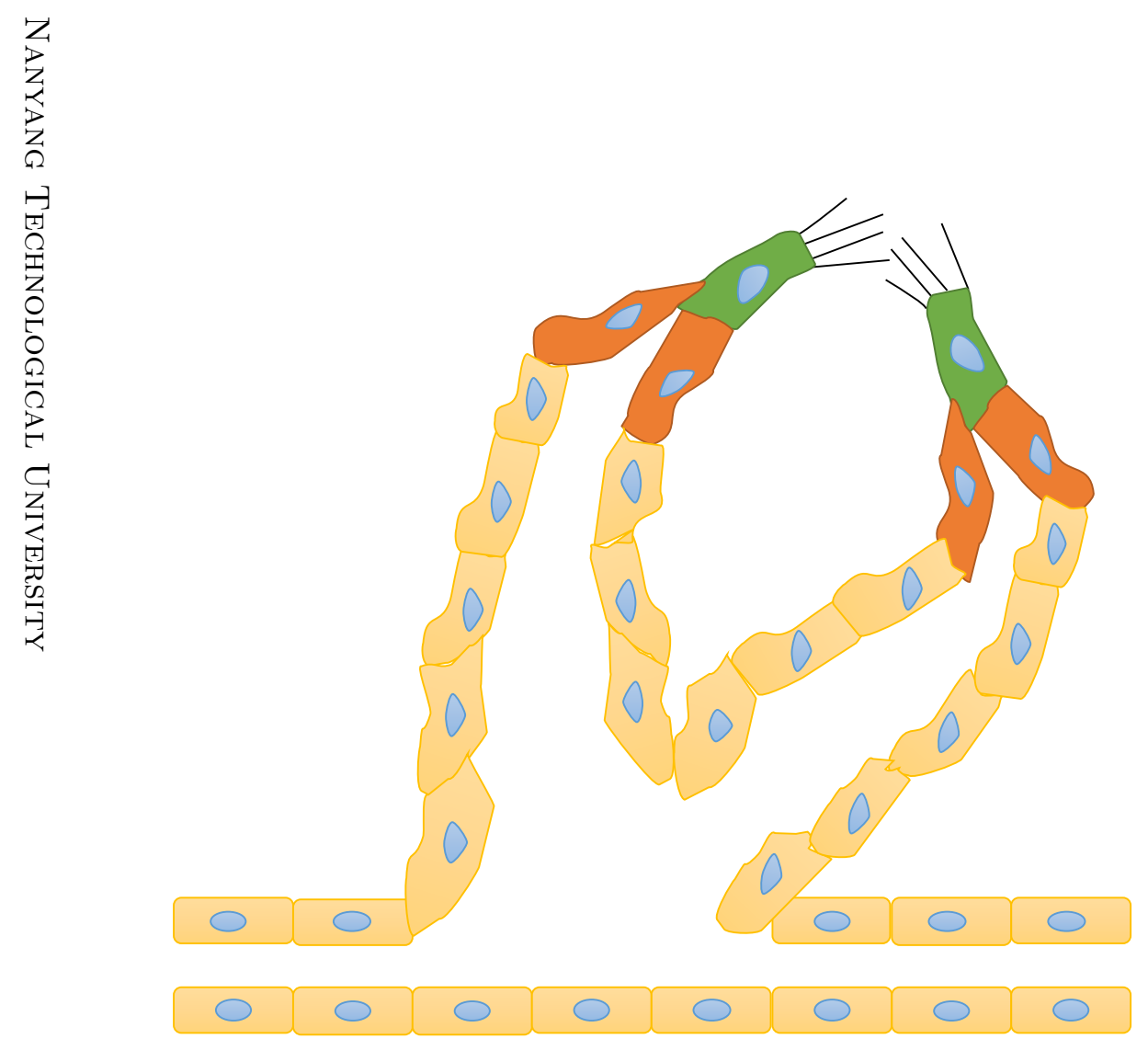

(e) Vessels undergoing reconnection.

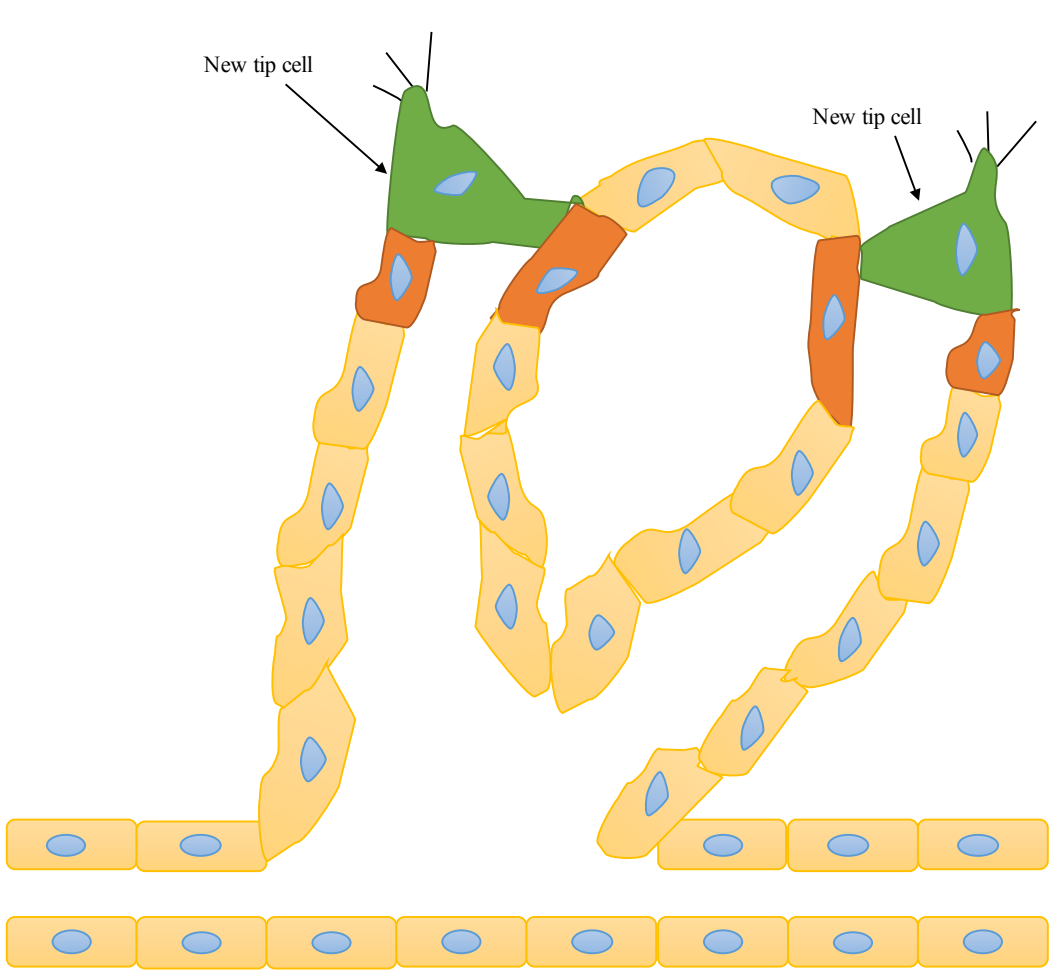

(f) Vessels after anastomosis.

Figure 1.2: Angiogenic sprouting process: (a) activation of ECs by TAFs; (b) tip cell selection via VEGF/Notch signaling pathways; (c) vessel elongation; (d) branching; (e) vessels undergoing anastomosis; and (f) vessels after anastomosis. 
formed branch is as labeled in Fig. 1.2(d). Anastomosis, also known as reconnection, is the formation of loops which occurs at a later stage after branching has taken place $[22,23]$. In anastomosis, one tip cell fuses with either a tip cell or a stalk cell in another established sprout to form a closed loop. In this process, tip cells might transit to stalk cells and vise versa [51]. Fig. 1.2(e) illustrates the first scenario, where two tip cells are communicating through filopodia for the future reconnection. When the reconnection is completed, a close loop is formed, as shown in Fig. 1.2(f). The two former tip cells would have converted to stalk cells and two new tip cells are leading the way.

The newly formed vascular network is said to be mature after many stages of sprouting, branching, and reconnection [6, 52].

\subsection{Motivations and Objectives}

Over many years, most of the angiogenic experiments have been performed in in vivo system. Although it provides the right environments, it is difficult to interpret the observations due to the complex nature. Alternatively, the traditional $2 \mathrm{D}$ in vitro cell culture is a simpler system, but significantly lacks many essential conditions compared to the actual in vivo system. For instance, the angiogenic networks cultured in $2 \mathrm{D}$ in vitro systems do not include vessels/lumens for blood to flow through. Moreover, the 2D cell signaling in these setups can not represent the real cell environments. The 3D microfluidic devices (MFDs), with channels allowing either fluid to flow or gel to be injected, mimic the in vivo environments $[2,53]$. The cells has been proved to migrate in a 3D volume (see Fig. 2.3) in the MFDs to form 3D angiogenic lumens (see Fig. 2.6 to 2.9). The development of 3D MFDs makes it possible to observe both individual cells and the structure from the in vitro experiments.

Fig. 2.2 illustrates the 3D MFDs used in our angiogenic experiments, which al- 
low for easy microscopy observation of long-term cell culture and vascular formation [2]. In order to investigate the long-term cell behaviors (e.g. cell migration and cell phenotypic changes) in angiogenesis, we first need to culture angiogenic vessels with branching and reconnection in MFDs. Human microvascular endothelial cells (HMVECs) are more angiogenic-like compared to human umbilical vein endothelial cells (HUVECs) as HMVECs are microvascular cells. However, existing angiogenic studies mainly use HUVECs since HMVECs are more fastidious in the culture environments which makes long-term culture more challenging [54]. To the best of our knowledge, S1P has been shown to increase length of the sprouts when culturing HUVECs in in vitro angiogenic experiments [55, 56], but there are few studies which cultured and observed angiogenic vessels with branching using HMVECs in 3D in vitro experiments. Thus, our first objective is to observe angiogenic vessels with branching after long-term culturing of HMVECs in 3D MFDs and investigate the influence of S1P on the HMVECs in angiogenesis.

Time-lapse imaging is a valuable tool for studying cell behaviors $[57,58]$. It yields data of finer resolution than traditional "still-shot" studies and allows direct examination of cell dynamics [59]. Although staining allows better visualization of cells under fluorescence/confocal microscopy, it inhibits cell proliferation and migration especially for a long-term cell culture [60]. Our goal is to investigate the long-term behaviors of ECs in angiogenic sprouting. Hence, we culture the unstained ECs and observe the angiogenic vessels with a phase contrast microscope [61].

EC migration is a key component to angiogenesis [62]. Accurate cell tracking would lead to a better understanding its mechanism, which may facilitate the investigation of novel therapeutic measures by controlling the ECs migration in angiogenesis. Manual cell tracking from the in-vitro microscopy images is time-consuming, especially for large numbers of cells. Hence, an automated cell tracking tool is required to produce faster and more reliable analysis of long-term cell migration behaviors from the experimental observations. Conventional automated image processing systems for cell tracking mainly focus on various microscopy images from $2 \mathrm{D}$ in vitro 
cell culture systems $[63,64,65]$ and florescence/confocal images from 3D in vitro cell culture systems [66]. Cells in 2D culture systems form a thin monolayer and have clear boundaries. For cells in 3D culture systems, they are stained thus also have clear boundaries in florescence/confocal images. However, the ECs in our images do not have clear boundaries due to the existence of tight cell junctions. Moreover, the interval for our phase contrast image sequences is one day, which is much longer than most of the existing cell tracking systems. Therefore, tracking these cells are a challenge which has not been addressed previously.

Angiogenic vessels are formed through the coordinated ECs migration, and the dynamic inter-transition of cell phenotypes. However, the quantitative understanding of the vessel formation, which is regarded as a simple form of collective cell behavior, is still missing. Moreover, the dynamic selection and continued competition of cell phenotypes, which would be useful for unraveling its biological function and developing computational models, is still not fully understood [15].

Therefore, the objectives of this thesis are to:

- observe angiogenic vessels with branching after long-term culturing of HMVECs in 3D MFDs, and investigate the influence of S1P on angiogenesis,

- propose an automated multi-cell tracking system to track the migration of ECs within the angiogenic vessels from the time-lapse phase contrast images, and generate cell lineage plots,

- develop an automated image analysis tool to track the formation of the angiogenic vascular networks, and identify cell phenotypes from the time-lapse phase contrast images.

The proposed automated image analysis systems are expected to help biologists to compare the influences of different growth factors on angiogenic vessels quantitatively and accurately, leading to a better understanding of angiogenic mechanisms. 
Moreover, these systems generate numerical data to build computational models to predict collective cell behaviors (e.g. multi-cell migration) and vascular formation in angiogenesis.

\subsection{Thesis Organization and Overview of Contri- butions}

The thesis is organized as follows. In Chapter 2, we elaborate on the procedures of the angiogenic experiments that we conduct in 3D MFDs. Specifically, we provide an overview of the structure and preparation of the MFDs, and the cell seeding process. A VEGF gradient is created to initialize the angiogenic sprouting by activation of the HMVECs. By adding S1P in the angiogenic experiments, we observe angiogenic vessels with branching after long-term culturing of HMVECs. The sprouting processes are recorded with a phase contrast microscope once a day and with a confocal microscope at the end time. Examples of different types of experimental images are provided in this chapter. These acquired time-lapse phase contrast images are used for the development of the automated image analysis systems to quantitatively analyze ECs migration and vascular formation. In order to study the influence of S1P on angiogenic vessel morphologies, we apply three different S1P conditions as listed in Table 2.1. The experimental observations suggest that the vessels formed under the positive S1P gradient are longer than the other two conditions. To our best knowledge, we are the first to investigate the influence of S1P on the angiogenic vessel morphologies formed by culturing HMVECs in MFDs over long time period.

In Chapter 3, we propose an automated multi-cell tracking system (AMCTS) that detects and tracks the migrating ECs within the angiogenic vessels from the timelapse phase contrast images. AMCTS consists of preprocessing to remove the non-gel region and extract the binary shape for the angiogenic vessels, cell detection to detect and label EC candidates, and multiple hypothesis Kalman filtering to associate and 
track the detected EC candidates over time. This is the first system to detect and track the unstained cells in in vitro 3D structures from phase contrast images. By applying partial least square regression (PLSR) to extract discriminative features from the training templates, we distinguish the ECs from background accurately. With the availability of the end-point confocal images with accurate cell locations, we initialize our tracks with the final time point after all proliferations have occurred, whereas other related cell tracking applications start with the image at the first time point. Furthermore, we incorporate biological knowledge to estimate the track probability during cell association. Numerical results indicate that AMCTS is able to track the cell migration trajectories accurately. Cell lineage plots, showing the history of the cell proliferation and cell migration, are also generated automatically from this system. By comparing with the identified biomarkers in the end-point confocal images, biologists can explore links between the chemical and physical characteristics of a cell. With this information about the cell history, mathematical models can be developed to predict the cell migration under different conditions, leading ultimately to a better understanding of the angiogenesis processes.

In Chapter 4, we develop an automated vessel formation tracking system (AVFTS) to track the angiogenic vascular formation, to extract the quantitative vessel information, and to identify the cell phenotypes from the time-lapse phase contrast images. AVFTS consists of preprocessing, skeletonization, and branch tracking. We firstly apply the preprocessing step, which is identical to the preprocessing step in AMCTS, to obtain the binary vessel shapes. Next, we extract the vessel skeletons by a distance transform (DT) and an augmented fast marching method (AFMM). The extracted vessel skeletons are segments connected by branch nodes and end nodes. We then distinguish and remove the specious skeleton segments which are not part of the vessels by a binary support vector machine (SVM) classifier. Lastly, we identify the route for each branch by joining the linked skeleton segments and associate the branches over image sequences by the Hungarian method. AVFTS is a novel system to track the angiogenic vessel formation in $3 \mathrm{D}$ in vitro cell culture 
systems. It extracts quantitative branch information including the length and width of each branch, and the number of branches for each sprouting region. By incorporating the branch information with the EC locations obtained from AMCTS, we distinguish tip and stalk cell phenotypes from the images, which would be useful for unraveling the biological functions of different cell phenotypes. Moreover, we apply AVFTS to quantify the angiogenic vessel morphologies under different S1P conditions, which significant saves biologists' time compared with manually measuring the morphological parameters. From the quantitative results, we conclude that the positive S1P gradient increases ECs migration and vessel elongation, and leads to higher possibilities for branching to occur.

In Chapter 5, we conclude the thesis by summarizing our contributions and suggesting possible directions for future works. 


\section{Chapter 2}

\section{Experimental Materials and Methods}

Summary - In this chapter, we present the procedure of the angiogenic experiments conducted in 3D microfluidic devices (MFDs). We begin this chapter with the introduction of the MFDs in Section 2.1. We then describe the MFDs fabrication process in Section 2.2. In Section 2.3, we culture HMVECs in MFDs under different S1P conditions over 10 to 14 days to grow angiogenic vessels with branching and to investigate the effect of $S 1 P$ on angiogenic vessels morphologies. In Section 2.4, we provide examples of the angiogenic vessels in different types of microscopy images. The phase contrast image sequences obtained under $20 x$ magnifications are used for the development of our automated image analysis systems.

\subsection{Introduction of MFDs}

Over the past years, most of the angiogenic experiments have been performed in in vivo systems, such as mouse models [67] and zebrafish models [68]. Although they provide the right environments, it is difficult to interpret the observations due 
to their complex nature and imaging difficulty. Alternatively, 2D cell culture in a static dish (Fig. 2.1(b)) is a classically preferred model since it is easily controlled, observed, measured, and manipulated. However, it significantly lacks many essential conditions from the in vivo system. For instance, ECs in 2D in vitro angiogenic experiments cannot form vessels.

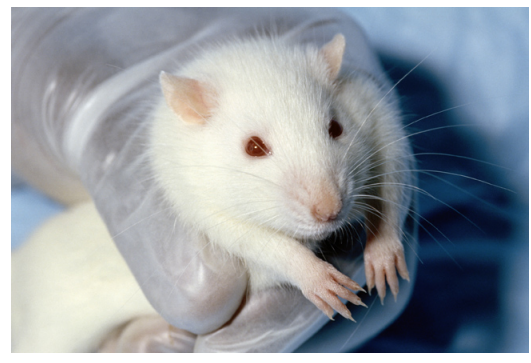

(a) Animal testing [69].

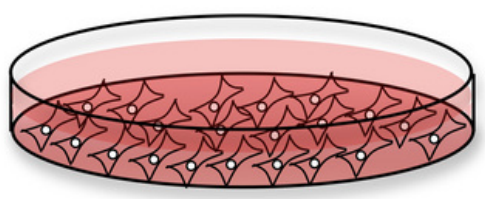

(b) 2D cell culture [70].

Figure 2.1: Examples of conventional in vivo and in vitro models for angiogenesis studies.

Our angiogenic experiments are conducted in the "high throughput" MFDs, as illustrated in Fig. 2.2(a) [2]. MFDs, with channels allowing either fluid to flow or gel scaffolds to be injected, mimic the in vivo environments and make it possible to observe both the individual cells and the structure from in vitro experiments. The advantage of the microfluidic platform is that it provides a well-controlled cell culture environment which can closely represent in vivo situations and can be observed easily in real time [71]. As controlled reactions can be reproduced with a small volume of samples and reagents, MFDs are used nowadays for long-term studies of many biological processes including angiogenesis.

Fig. 2.2(b) illustrates the internal structure of the MFD used in our experiments, where channel A, B, and C are media filling channel, cell seeding channel, and gelfilling channel respectively. Each MFD has 37 sprouting regions separated by posts. The posts are designed to cage the collagen gel (when injected) inside the gel channel. They are also used as features for registering the experimental image sequences. Fig. 2.2(c) illustrates an individual sprouting region. The sprouting processes can be observed with a phase contrast microscope and a confocal microscope. 


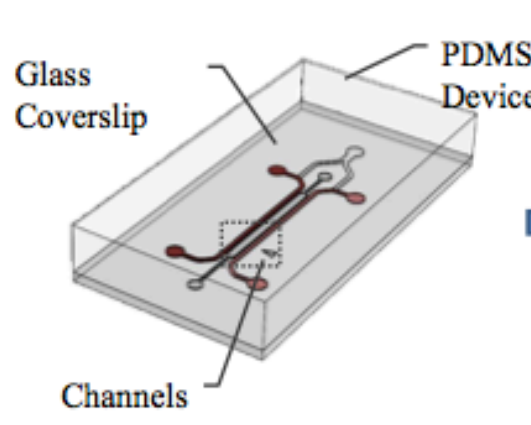

(a)

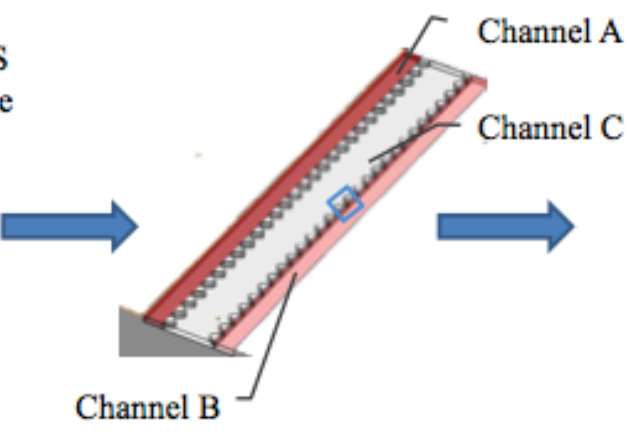

(b)

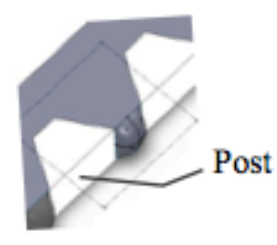

(c)

Figure 2.2: "High throughput" MFD [2]: (a) external appearance; (b) internal appearance with 37 sprouting regions; and (c) individual sprouting region.

In order to prove that the ECs migrate in 3D environments inside the MFDs, we compared the cell migration distances in both $x y$-plane and $z$-direction based on the confocal images from angiogenic sprouting experiments. The total experimental time for this migration study is 12 hours, and the imaging time interval is 30 mins. For each EC, Fig. 2.3(a) compares its migration distance, which is the position difference between the start and end time, in both $x y$-plane and $z$-direction. As ECs may move forward and backward, the maximum cell migration distances regardless of time are also compared, as shown in Fig. 2.3(b). The ECs migrate over large distances in all three directions, as can be seen from Fig. 2.3. Therefore, it is clear that the ECs migrate in a 3D volume in the MFDs.

\subsection{MFDs Fabrication}

MFDs are made of Polydimethylsiloxane (PDMS - Sylgard 184 at a ratio of 10:1 polymer to cross-linker, Dow Corning Corporation) in the negative patterned wafers designed by Farahat et al. [2]. The devices are detached from the wafers, autoclaved in deionized water, and dried overnight in an oven at 70 degrees. Next, the devices are plasma bonded to $22 \mathrm{~mm} \times 60 \mathrm{~mm}$ sterile glass slides to form closed microfluidic channels (channel A, B, and C in Fig. 2.2(b)). These channels are coated with 


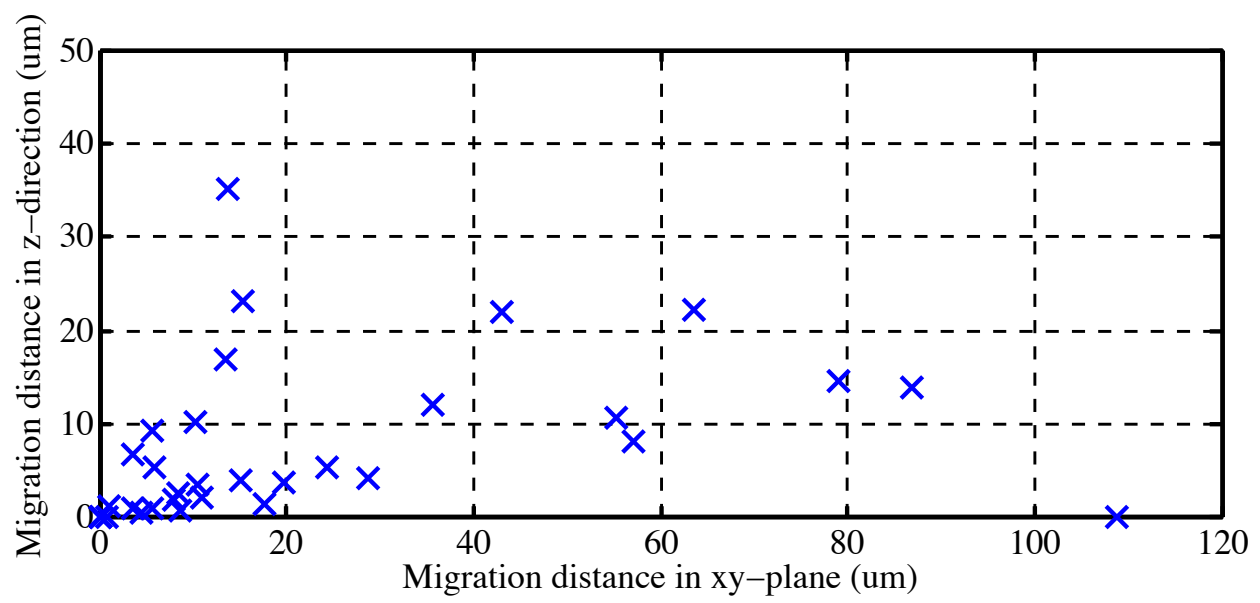

(a) Final cell migration distance.

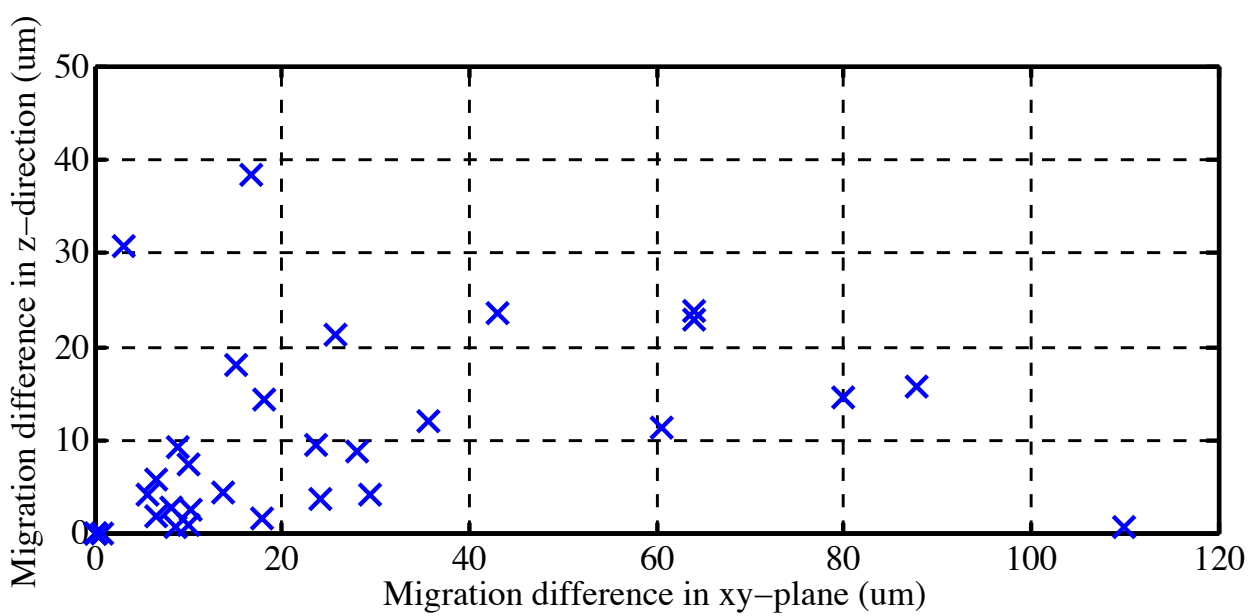

(b) Maximum cell migration distance.

Figure 2.3: Comparison of the migration distances of EC nuclei in $x y$-plane and $z$-direction in the MFDs.

poly-d-lysine (PDL - P7886, Sigma) for four hours, washed with sterile water, and dried overnight to render the hydrophilic channel surface hydrophobic. This allows the filled gel to be confined within the gel channel. Type I collagen (354236, BD Biosciences) is injected into the gel-filling channel (channel C in Fig. 2.2(b)) and polymerized for one hour in a humidity box in incubator. After filling the media filling channel (channel A in Fig. 2.2(b)) and cell seeding channel (channel B in Fig. 2.2(b)) of the MFDs with Endothelial Cell Growth Media (Lonza EGM-2MV Cat. No. CC-3202) [71], these devices are ready for cell seeding. 


\subsection{Experimental Conditions}

Most of the angiogenic studies use HUVECs because they are easy to culture. HMVECs are more fastidious in the culture environments which makes long-term culture more challenging [54]. However, as HMVECs are microvascular cells, they have more angiogenic-like behaviors than HUVECs [72]. Thus, we culture HMVECs in our angiogenic experiments.

HMVECs (Lonza Cat. No. CC-2543) were received at passage 3 and expanded to passage 5 in EGM via standard mammalian adherent cell culture protocols, and then cryogenically frozen until needed. When cells were needed prior to seeding in a device, a passage 5 vial was thawed and expanded to passage 7 . When the cells reached 80-90\% confluence, the cells were trypsinized (0.05\% Trypsin EDTA, Invitrogen), centrifuged, and then suspended in EGM to a density of 2.5 million cells $/ \mathrm{mL}$.

During cell seeding, channel B is filled with the cell suspension. These cells will attach to the collagen gel and form a monolayer. Channel A is filled with EGM only.

After HMVECs are confluent in channel B, condition media are filled in both channel A and channel B to initialize the angiogenic sprouting. As discussed in Section 1.1, ECs tend to migrate towards a higher gradient of VEGF. Thus we create a VEGF gradient across the gel by providing media with high VEGF concentration in channel A (40 ng/ml) and low VEGF concentration in channel B $(20 \mathrm{ng} / \mathrm{ml})$. About two days after seeding, ECs in the monolayer specialize into tip and stalk cells, migrate out, and form new angiogenic sprouts.

As explained, our goal is to investigate the long-term behaviors of ECs in angiogenic sprouting. Hence, we culture the unstained ECs and observe the angiogenic vessels with a phase contrast microscope daily over a period of 10 to 14 days. At the end time, we image the sprouts with a confocal microscope after staining cell nuclei with 


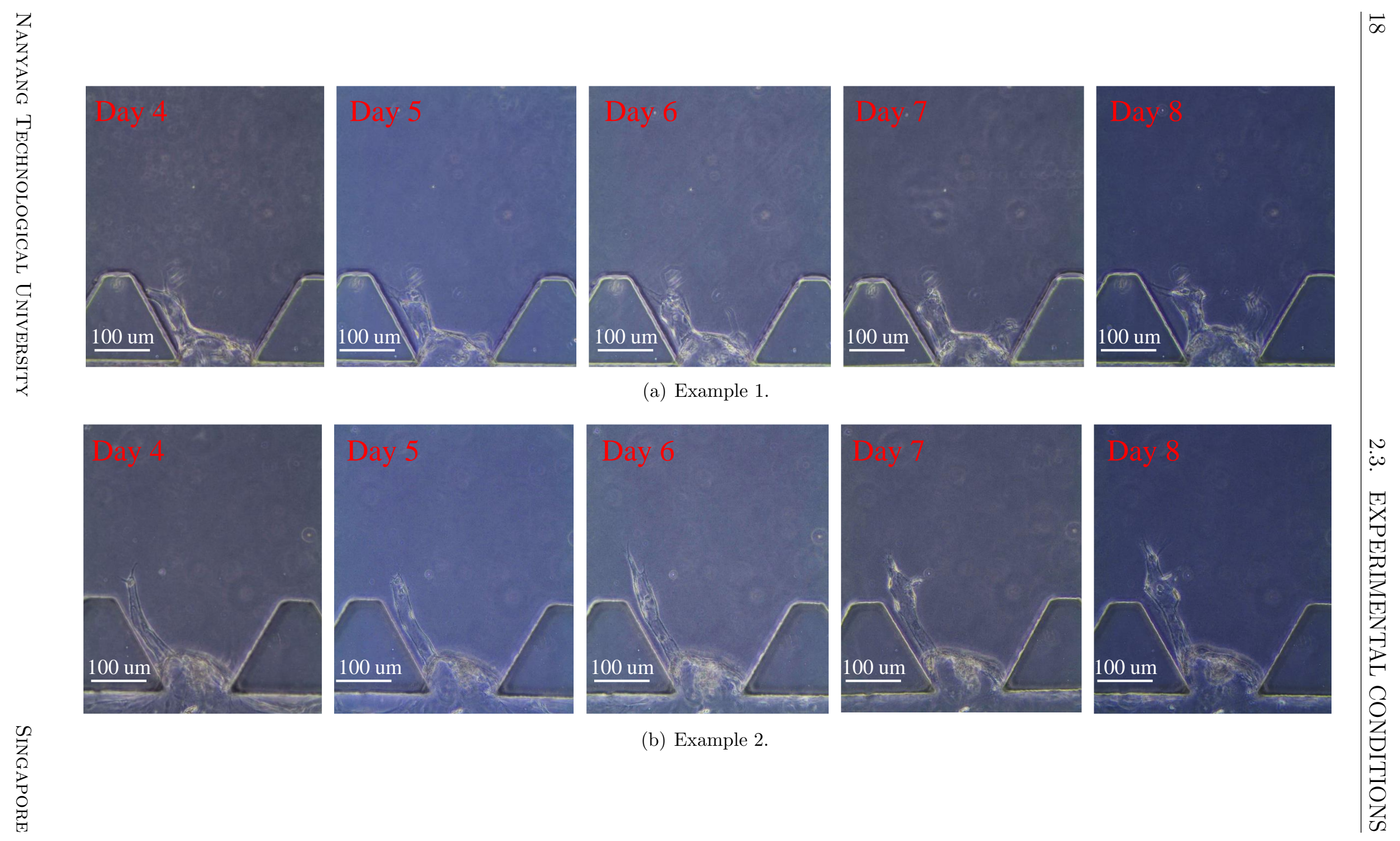



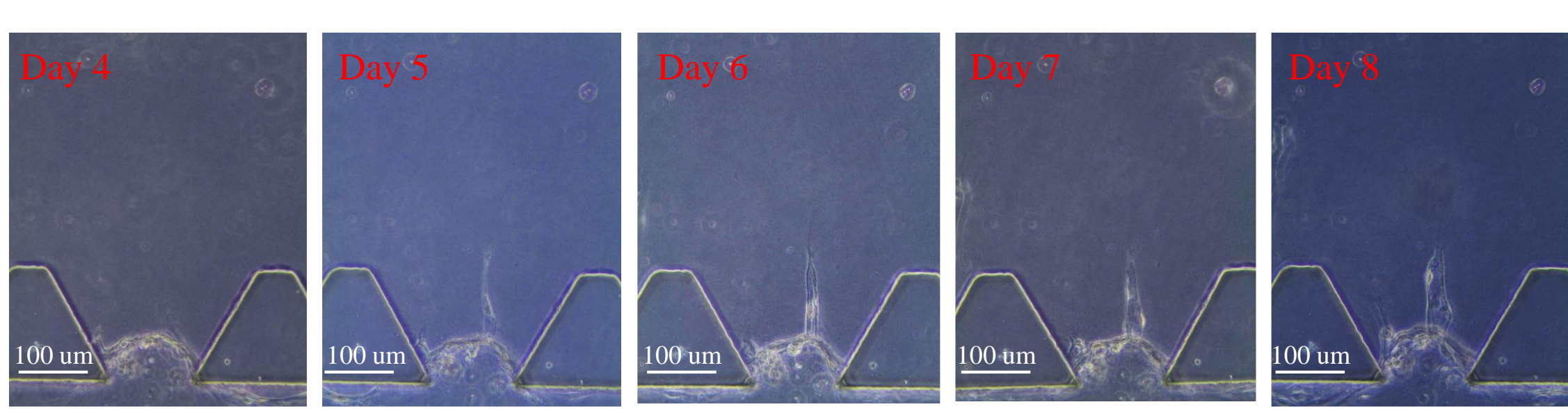

(c) Example 3.
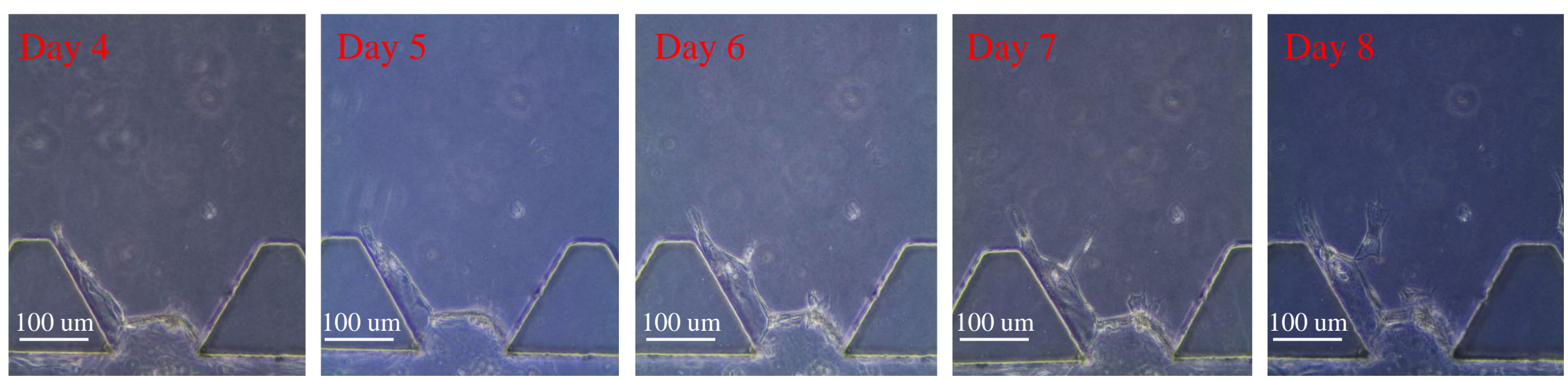

(d) Example 4.

Figure 2.4: Examples of angiogenic vessels (without S1P) in phase contrast images for 5 consecutive days (from day 4 to day 8 after cell seeding). 
Hoechst dye (Invitrogen) and actin with Phalloidin 488.

We provide four examples of the angiogenic vessels in Fig. 2.4. Each example shows the phase contrast images of the vessels in a same sprouting region for 5 consecutive days (from day 4 to day 8 after cell seeding). From these images, we can observe that the ECs migrate and the vessels extend slowly, especially from the 7th day after seeding. Branching does occur in these experiments, but reconnection hardly happens.

As explained in Section 1.1, branching and reconnection are key processes to form a mature vascular. We are interested in long-term collective cell behaviors such as ECs migration, phenotypic changes, and branch formation. Thus, we firstly need to culture long angiogenic vessels which allow branching to happen. S1P has shown to increase the average vessel length by increasing the migration of HUVECs in $[55,56]$. Therefore, we add S1P in our angiogenic experiments.

We start with a S1P concentration of $250 \mathrm{nM}$ in channel A, which is same as the concentration used in [56]. Fig. 2.5 provides an example of the angiogenic vessels for 3 consecutive days, from which we can see that adding S1P does increase the vessel

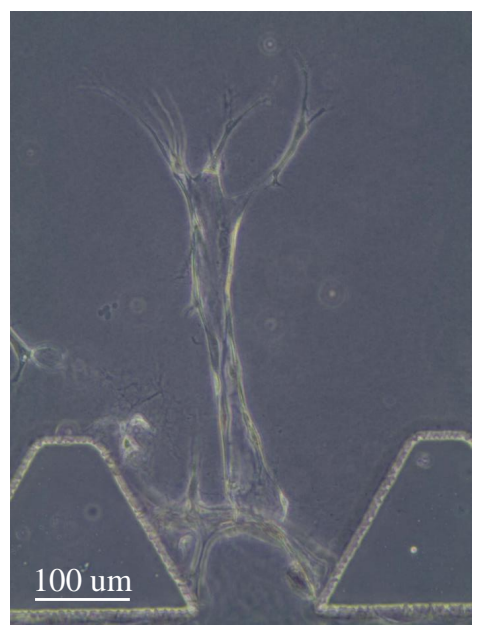

(a) Day 7.

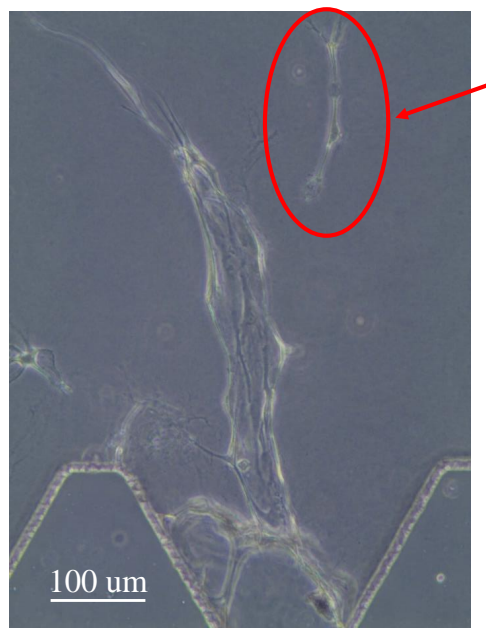

(b) Day 8.

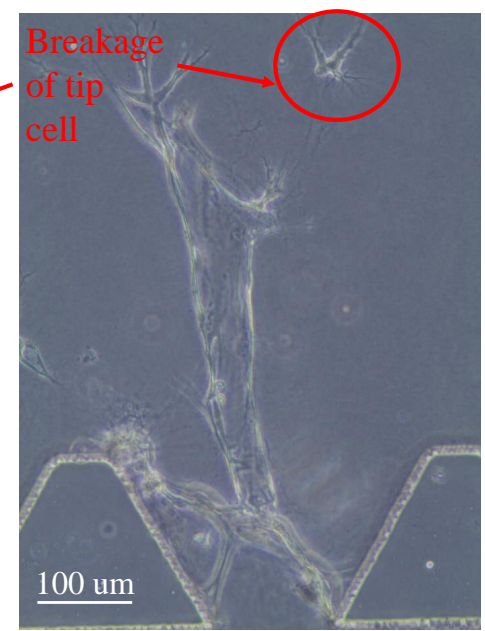

(c) Day 9.

Figure 2.5: Breakage of tip cell under high S1P concentration. 
length. But we also notice that some tip cells will migrate too fast while stalk cells cannot follow, leading to breakage of the tip cells from the sprouts, as labeled in Fig. 2.5. We hypothesize that S1P functions mainly on tip cells and HMVECs are more responsive to S1P than HUVECs. We reduce the S1P concentration to $31 \mathrm{nM}$ in order to form long angiogenic vessels with negligible breakage of tip cells.

By varying the S1P concentrations in the condition media filled in channel $\mathrm{A}$ and channel B, we create three different S1P experimental conditions, as shown in Table 2.1. We consider these three S1P conditions to further study the influence of S1P on angiogenic vessel morphologies. For all experiments, we apply a fixed VEGF condition (40 ng/ml in channel A and $20 \mathrm{ng} / \mathrm{ml}$ in channel B).

Table 2.1: Different S1P conditions in the angiogenic experiments.

\begin{tabular}{|c|c|c|}
\hline Experiment conditions & $\begin{array}{c}\text { S1P concentration in } \\
\text { channel A }(\mathbf{n M})\end{array}$ & $\begin{array}{c}\text { S1P concentration in } \\
\text { channel B (nM) }\end{array}$ \\
\hline No S1P (ctrl) & 0 & 0 \\
\hline Positive S1P gradient & 31 & 0 \\
\hline Negative S1P gradient & 0 & 31 \\
\hline
\end{tabular}

We have 12 devices for positive S1P gradient condition, 4 devices for negative S1P gradient condition, and 4 devices for no S1P condition respectively. Each device has 37 sprouting regions. We observe the angiogenic vessels in each sprouting region daily with a phase contrast microscope over 10 to 14 days.

\subsection{Angiogenic Experimental Observations}

From the angiogenic experiments, we acquired different types of microscopy images, including time-lapse phase contrast images under 4x magnification and 20x magnification, and the end-point confocal images. Examples of the different image types are shown in Fig. 2.6 to Fig. 2.8. 
Fig. 2.6(a) illustrates one phase contrast image of the MFD under 4x magnification. By stitching all the $4 \mathrm{x}$ phase contrast images of one MFD, we obtain an image of the angiogenic vessels within the entire device, as shown in Fig. 2.6(b). Fig. 2.6(a) corresponds to the red rectangular in Fig. 2.6(b). From Fig. 2.6, we can see that we are able to culture long vessels up to $1 \mathrm{~mm}$, and branching and reconnection frequently happens.

Each sprouting region of the MFD is observed under 20x magnification by a phase contrast microscope. Due to imaging constraints, several overlapping images are

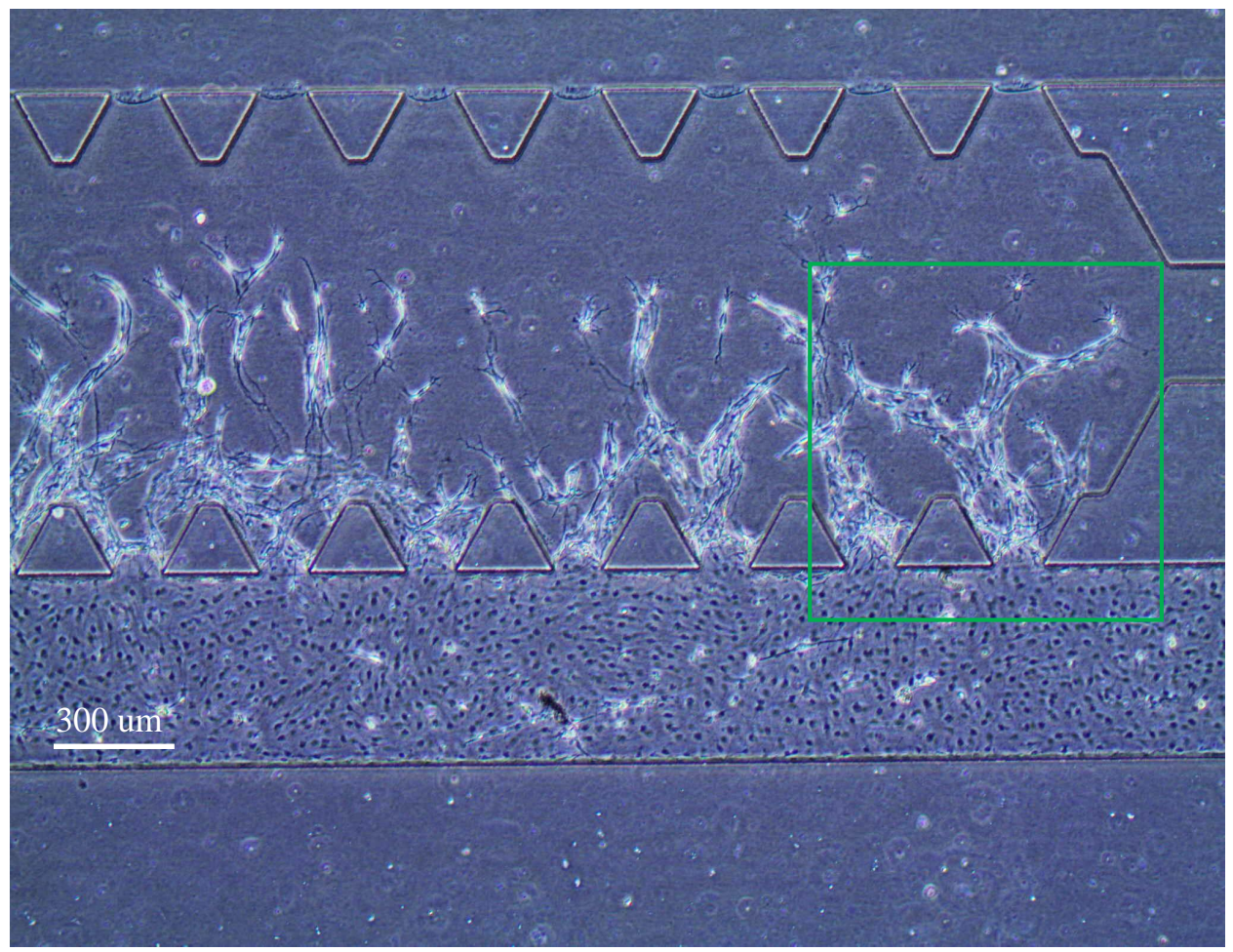

(a) Phase contrast image under 4x magnification: corresponds to the red box in (b).

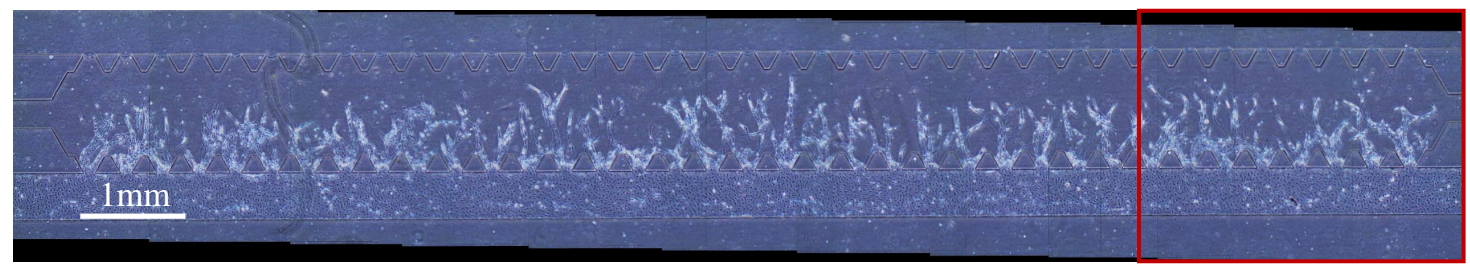

(b) View of angiogenic vessels within the entire MFD.

Figure 2.6: Example of angiogenic vessels in phase contrast image under 4x magnification. 


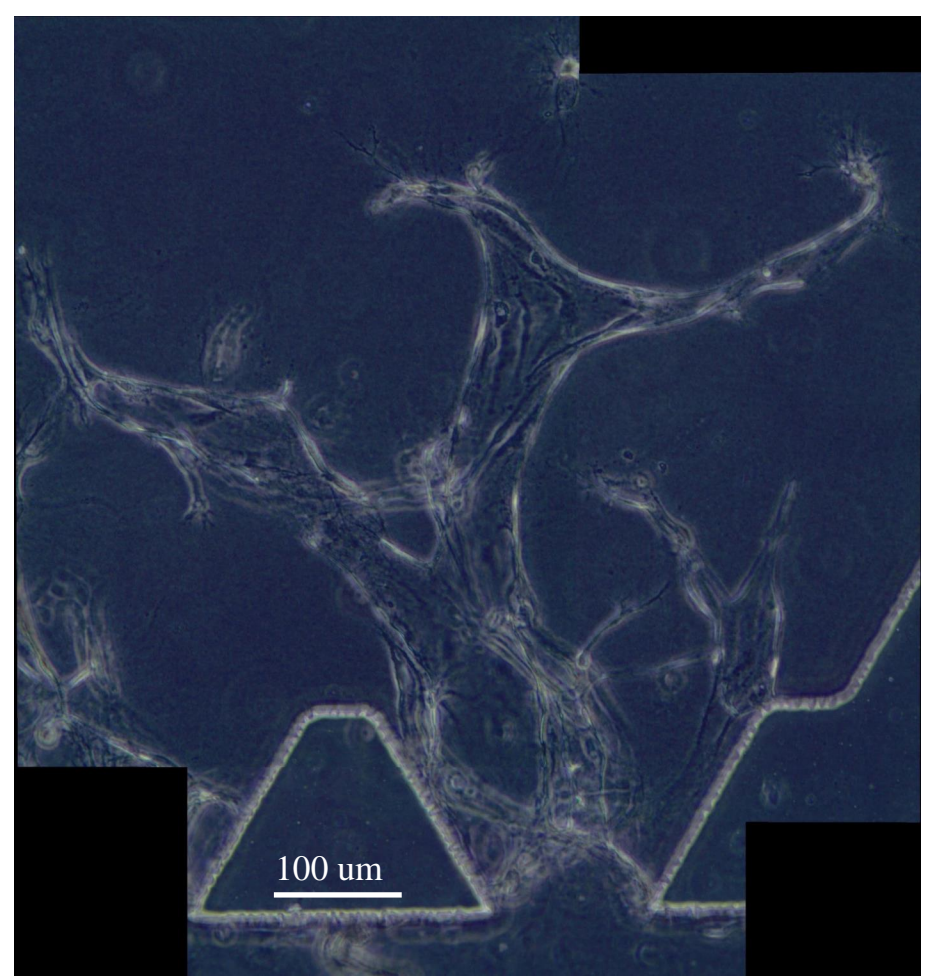

Figure 2.7: Example of angiogenic vessels in a sprouting region by stitching the overlapping phase contrast images under 20x magnification: corresponds to the green box in Fig. 2.6(a).

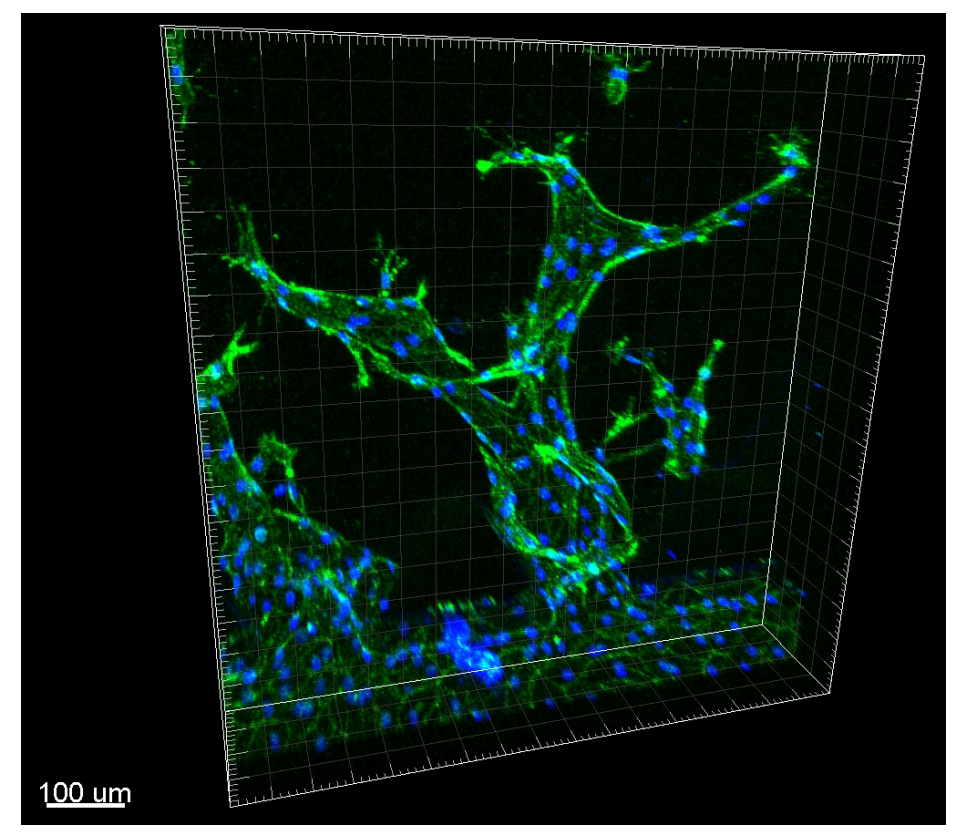

Figure 2.8: Example of angiogenic vessels in the same sprouting region as in Fig. 2.7 in the end-point confocal image: cell nuclei are in blue and actin is in green. 
required to capture a long vessel in one sprouting region. The full view of this long vessel can be obtained by stitching these overlapping images. For instance, Fig. 2.7 shows the angiogenic vessels in a sprouting region after stitching. It corresponds to the green box in Fig. 2.6(a). The stitching procedure will be discussed in Chapter 3 in detail. The time-lapse phase contrast images under 20x magnification are the images we are considering in this thesis.

At the end time, we image the angiogenic vessels with a confocal microscope after staining cell nuclei with Hoechst dye (Invitrogen) and actin with Phalloidin 488. Fig. 2.8 is a confocal image of the angiogenic vessels within the same sprouting region as in Fig. 2.7, where the cell nuclei are in blue and the actin is labeled in green.

Fig. 2.9 shows the section view of angiogenic vessels in MFDs in the confocal image. The "hole" in the $x z$-plane shows the lumen formed by the ECs, indicating that the ECs in MFDs can form 3D angiogenic lumens.

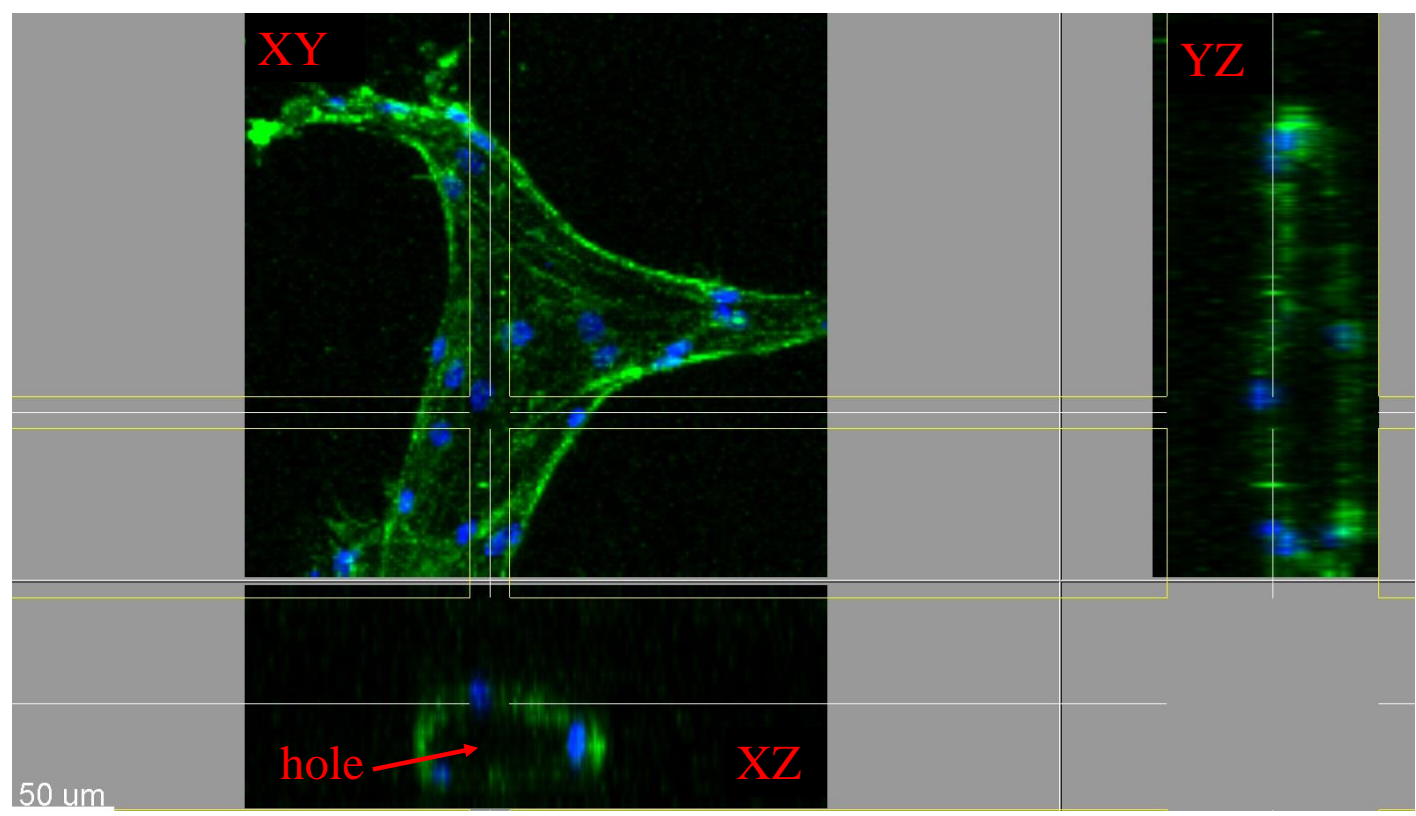

Figure 2.9: Section view of angiogenic vessels in MFDs in the confocal image. 


\section{Chapter 3}

\section{Automatic Tracking of Endothelial Cells in Angiogenic Vessels}

Summary - In this chapter, we develop an automated multi-cell tracking system (AMCTS) to track the migration of the ECs within angiogenic vessels. This system is a tracking by detection algorithm which firstly detects the EC candidates from the time-lapse phase contrast images and then links the detected EC candidates into tracks. AMCTS consists of preprocessing to remove the non-gel region and segment the binary vessel shapes, cell detection to identify the EC candidates, and multiple hypothesis Kalman filtering to associate and filter the identified EC candidates over image sequences. We incorporate the migration of ECs from monolayer and cell proliferation time to obtain more reliable cell association results. Since the endpoint confocal images provide accurate cell locations, we track the cells backward to generate cell migration trajectories and cell proliferation histories. We review the existing approaches for cell segmentation and tracking in Section 3.1. In Section 3.2, we elaborate on the proposed automated multi-cell tracking system. In Section 3.3, we present numerical results on our experimental phase contrast images. We offer concluding remarks in Section 3.4. 


\subsection{Introduction and Literature Review}

EC migration is a key component to angiogenesis [62]. Studies have shown that the directional EC migration is mainly influenced by chemotactic (gradient of soluble chemoattractants), haptotactic (gradient of immobilized ligands), and mechanotactic (mechanical forces) stimuli [73, 74]. The basic concepts of EC migration have been obtained mostly from observations of $2 \mathrm{D}$ in vitro cell culture systems. However, $3 \mathrm{D}$ in vitro environments (e.g., MFDs), where cells must integrate and coordinate with their surroundings to determine their migration, are more similar to the in vivo system. Quantitative analysis of the observations from 3D in vitro cell culture systems provides numerical information for a better interpretation of cell migration and angiogenesis [75, 76, 77].

The existing automated image analysis techniques for cell segmentation and tracking can be classified into two categories: tracking by model evolution and tracking by detection. The former category detects and tracks the cells simultaneously by representing the cells with mathematical models, whereas the latter category firstly detects cell candidates in all the frames independently and then links the detected candidates into tracks.

In tracking by model evolution algorithms, the mathematically represented cell contours are smoothly evolved from one time frame to the next one, using a velocity term defined by the content of the "target frame" [78, 79, 80, 81, 63]. In general, the tracking by model evolution algorithms require accurate cell contour/boundary detection and a high imaging frequency to allow sufficient cell overlaps between frames. These approaches are not applicable to our images due to the unclear cell boundaries (tight cell junctions) and low imaging frequency (one day).

In tracking by detection algorithms, cells are firstly detected in all the frames independently and then tracked by cell association. Cell detection approaches include thresholding of pixel intensities [82, 83], edge detection [84], image restora- 
tion [64, 85, 86], template matching [87], etc. Kanade et al. applied image restoration to segment the cells on a 2D flat surface from phase contrast images [64], as shown in Fig. 3.1(a). Our cell within the 3D angiogenic vessels are shown in Fig. 3.1(b), where the red arrows point to examples of cells. Due to the different culture environments, the cell morphologies are different. The thickness of a cell on a flat surface is around $3 \mu \mathrm{m}$ to $20 \mu \mathrm{m}$, whereas the thickness of the MFD is $120 \mu \mathrm{m}$. Due to the artifacts of phase contrast microscopy, imaging thicker specimens produces severe halo effects which reduce the visibility of cell boundaries. Thus, unlike the data in Kanade et al. [64], our cells do not have clear boundaries. In addition, some cells may be slightly out of focus. Therefore, the image restoration technique is not applicable to our images. A different approach for cell detection is template matching: a template is compared to patches of a gray level image by means of a match statistics evaluated for each patch [88]. Euclidean distance and mean square difference are commonly used as the template matching criteria for cell detection [87, 65, 89, 90, 91]. Since we can easily obtain cell templates from end-point phase contrast images by aligning the latter with their corresponding end-point confocal images, we apply template matching to detect cell candidates in the phase contrast images. To reduce the computational complexity, discriminative features of the cells are extracted from a set of templates using partial least square regression (PLSR), helping to distinguish

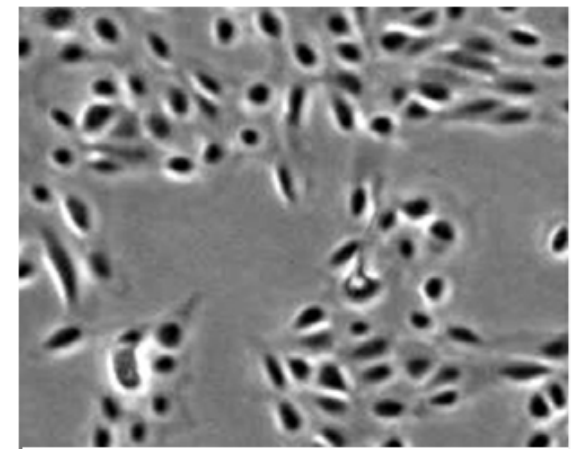

(a)

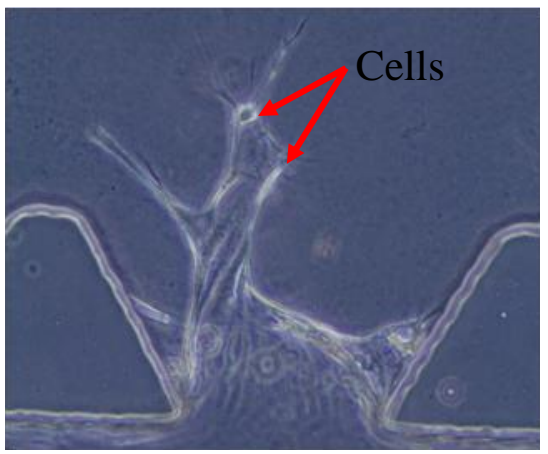

(b)

Figure 3.1: Examples of cells: (a) on a 2D surface and (b) within 3D vessels (red arrows point to cells). 
cells from background.

Subsequently, cell association algorithms are used to link the detected cell candidates into tracks. Cell association algorithms include "Nearest-neighborhood" association [92, 93], mean-shift process [94, 95, 96], Hungarian Method [97], Viterbi algorithm [98], Kalman filtering [99], particle filtering [100], Multiple Hypothesis Tracking (MHT) [64, 101, 102], Bayesian filtering [66], and active structured learning [103]. These techniques are either applied to images of cells migrating on a 2D surface or 3D in vivo florescence/confocal images, which are far different from our 3D in vitro application. Moreover, the interval for our image sequences is one day, which is much longer than most of the existing tracking systems. Therefore, tracking these cells is a challenge which has not been addressed previously.

\subsection{Automated Multi-Cell Tracking System}

We propose the AMCTS to track the ECs in angiogenic vessels, as illustrated in Fig. 3.2. We first preprocess the time-lapse phase contrast images to remove the non-gel region and segment the binary vessel shapes by stitching, image registration, and vessel segmentation. Next, we detect and label the EC candidates by template matching. Lastly, by combining backward Kalman filtering with MHT, we associate and track the detected EC candidates over the image sequences. From the tracking results, we obtain cell lineage and trajectory information for a better understanding of cell characteristics. In the following, we will discuss each component in detail.

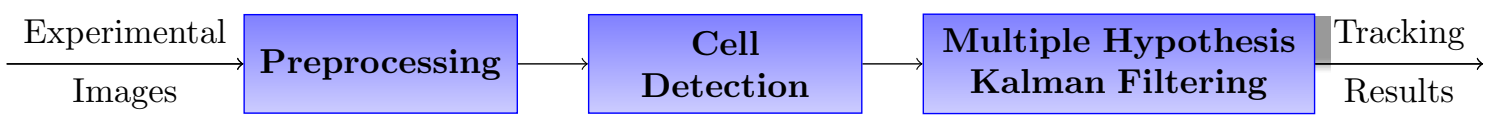

Figure 3.2: Diagram of the proposed automated tracking system: preprocessing to remove the non-gel region and segment the binary vessel shapes for all the aligned experimental images; cell detection to label all EC candidates; and multiple hypothesis Kalman filtering to associate the detected EC candidates. 


\subsubsection{Preprocessing}

There are three major steps in the preprocessing, leading to the binary vessel shapes: stitching, image registration, and vessel segmentation.

\subsubsection{Stitching}

Due to imaging constraints, several overlapping images are required to capture a long vessel in a sprouting region at 20x magnification. In Fig. 3.3(a) to 3.3(d), we provide examples of overlapping phase contrast images of angiogenic vessels in the same sprouting region over 4 consecutive days (day 9 to day 12).

Our first step is to construct a full view of the long angiogenic vessels by stitching the overlapping images. Speeded Up Robust Features (SURF) are employed as the features to find and match the corresponding points for stitching [104]. The stitched results of the overlapping images in Fig. 3.3(a) to 3.3(d) are shown in Fig. 3.4(a) to 3.4(d) respectively.

\subsubsection{Image Registration}

We acquired images manually at different times. Therefore, the angiogenic vessels are misaligned across the image sequences. As illustrated in Fig. 3.4(a) to 3.4(d), the locations of the posts in these images are different. To spatially align all the timelapse images in a sprouting region and transform them into one coordinate system, we apply an image registration algorithm with the posts as references [105]. This step is critical for tracking ECs migration and for investigating the vessel formation.

In order to register the images, we first extract salient features from these images. We choose the red points in Fig. 3.5 as the features. These points are the intersections of the straight post edges (line 1 to line 4). The Hough Transform (HT) 


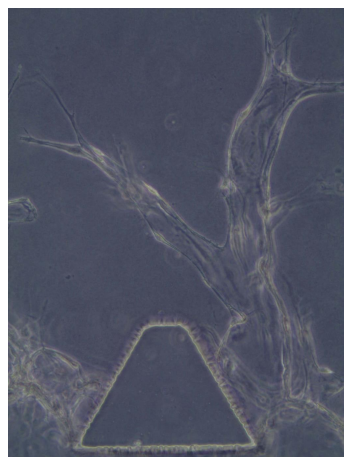

(a) Acquired images I.

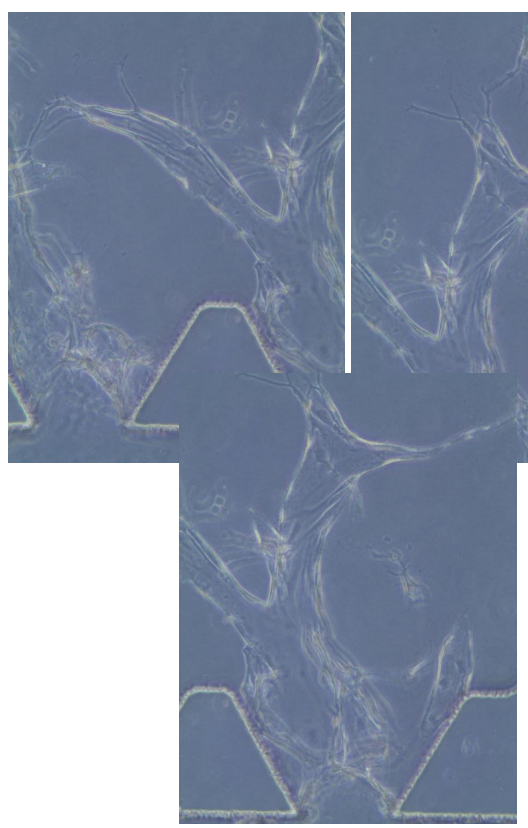

(c) Acquired images III.

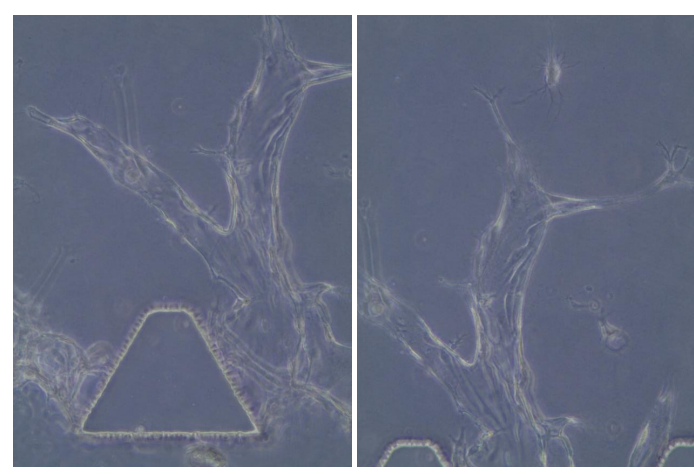

(b) Acquired images II.

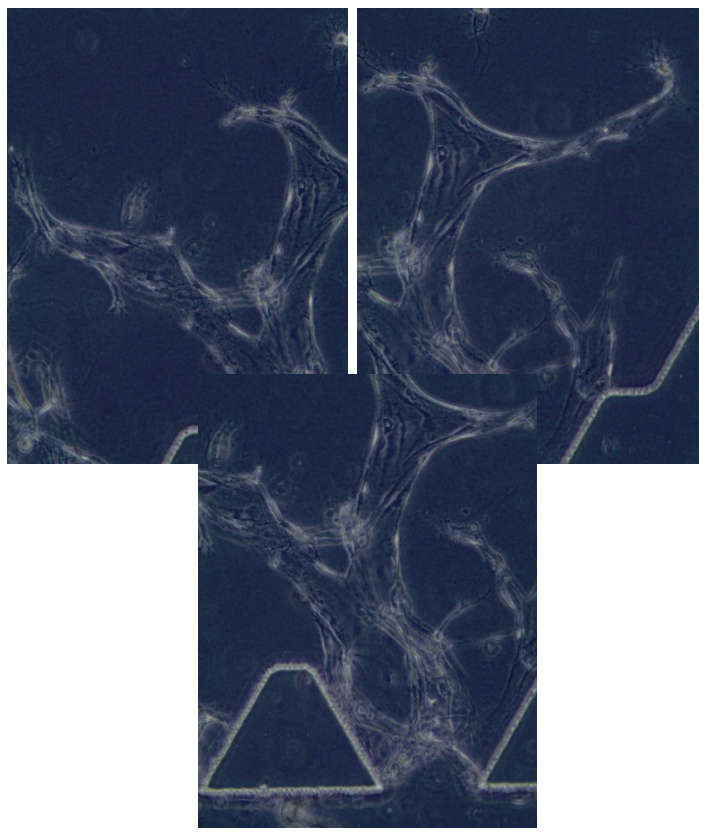

(d) Acquired images IV.

Figure 3.3: Examples of overlapping phase contrast images for angiogenic vessels in a same sprouting region over 4 consecutive days (day 9 to day 12). 


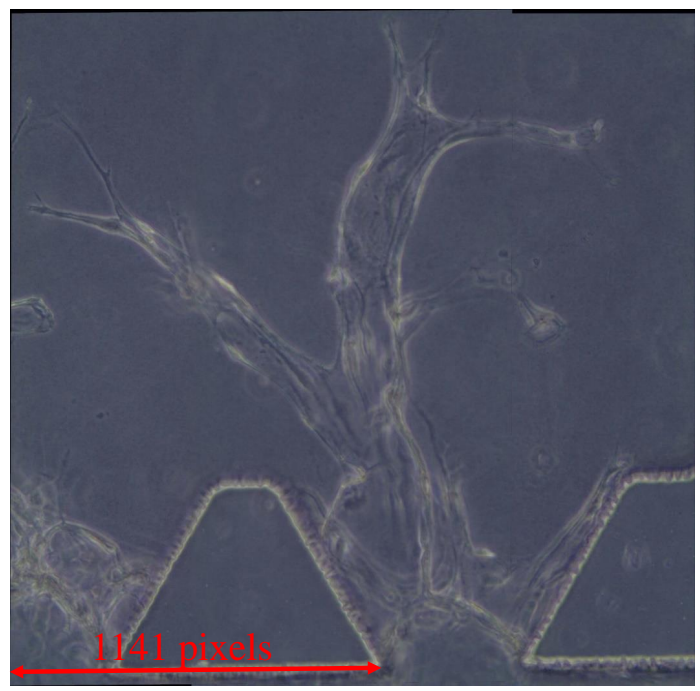

(a) Stitched image I.

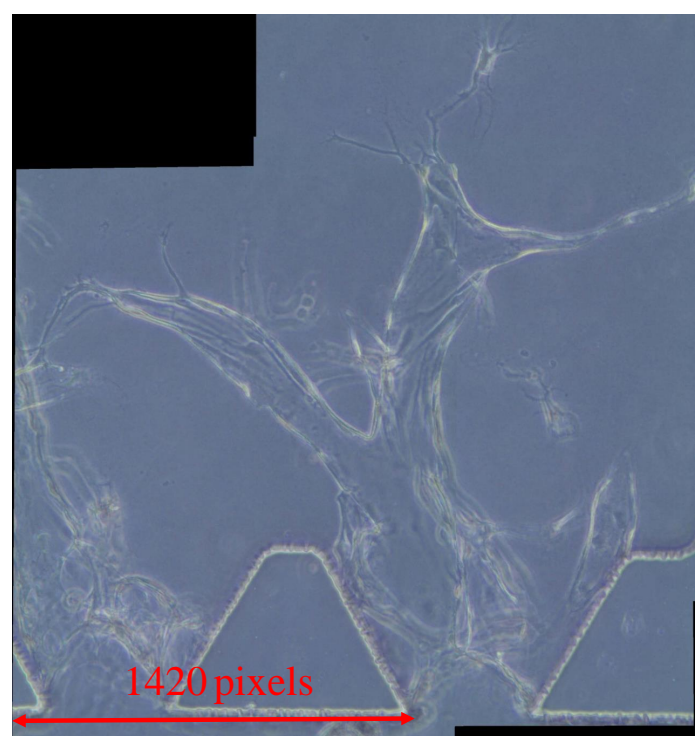

(c) Stitched image III.

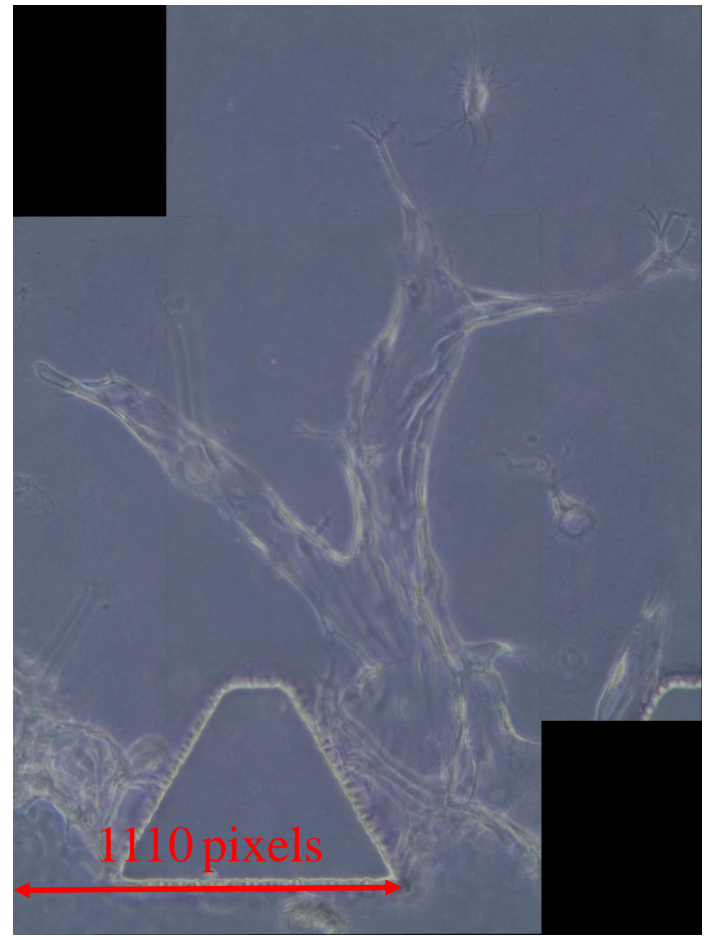

(b) Stitched image II.

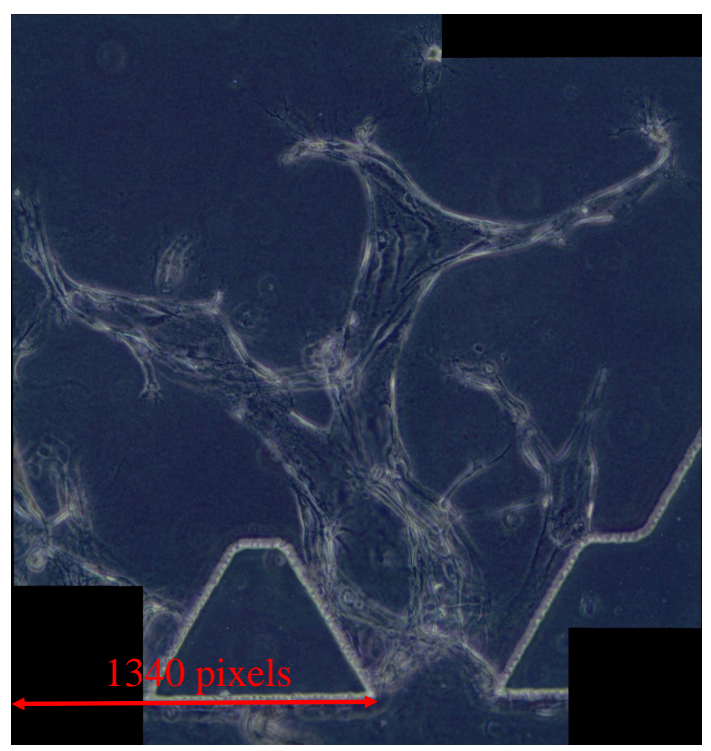

(d) Stitched image IV.

Figure 3.4: Stitching results for the examples in Fig. 3.3 showing the entire angiogenic vessels in a same sprouting region over 4 consecutive days (day 9 to day 12). 


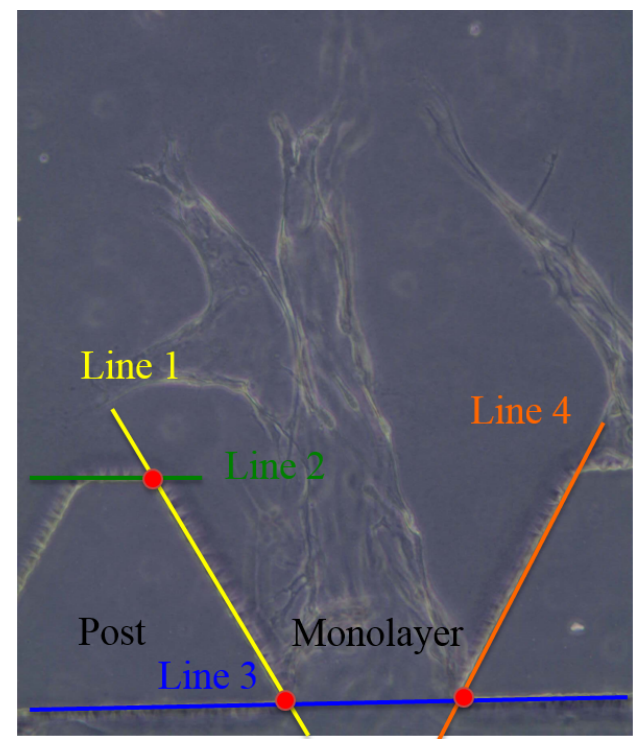

Figure 3.5: Features for image registration: line 1 to line 4 are represented by the Hough Transform, and the red points are the correspondence points.

is applied to automatically identify the straight lines to represent the edges of the posts [106]. In image space, a straight line can be described as:

$$
y=m x+b,
$$

where $m$ is the slope of the line, and $b$ is the $y$-intercept. Since $m$ is infinite for a vertical line, a line can be represented by:

$$
x \cos \theta+y \sin \theta=\rho,
$$

where $\rho$ is the length of the normal from the origin to this line, and $\theta$ is the orientation of the normal with respect to the $x$-axis (see Fig. 3.6(a)). By comparing equation (3.1) and (3.2), it is clear that all points $\left(x_{i}, y_{i}\right)$ on a line in the image fall into the same point $\left(\rho_{i}, \theta_{i}\right)$ in the parametric $\rho-\theta$ space [107].

The procedure to detect a line from an image using HT is as follows:

- Estimate the range of $\rho$ and $\theta$ for this line,

- Divide the parametric space into cells within the estimated range of $\rho$ and $\theta$, 
as shown in Fig. 3.6(b),

- Initialize each cell with zero: $A(\rho, \theta)=0$,

- For each detected point $\left(x_{i}, y_{i}\right)$ in the image, perform $A(\rho, \theta)=A(\rho, \theta)+1$ for all cells with $\rho$ and $\theta$ satisfying the equation (3.2).

The final value of $A(\rho, \theta)$ reflects the number of points on the line $\rho=x \cos \theta+y \sin \theta$ in the image. We choose the $\rho$ and $\theta$ which corresponds to the largest $A(\rho, \theta)$ value to represent the line we want to detect.

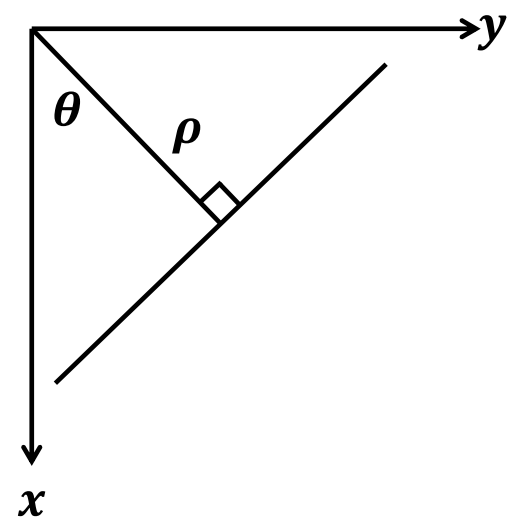

(a) Parametric description of a straight line.

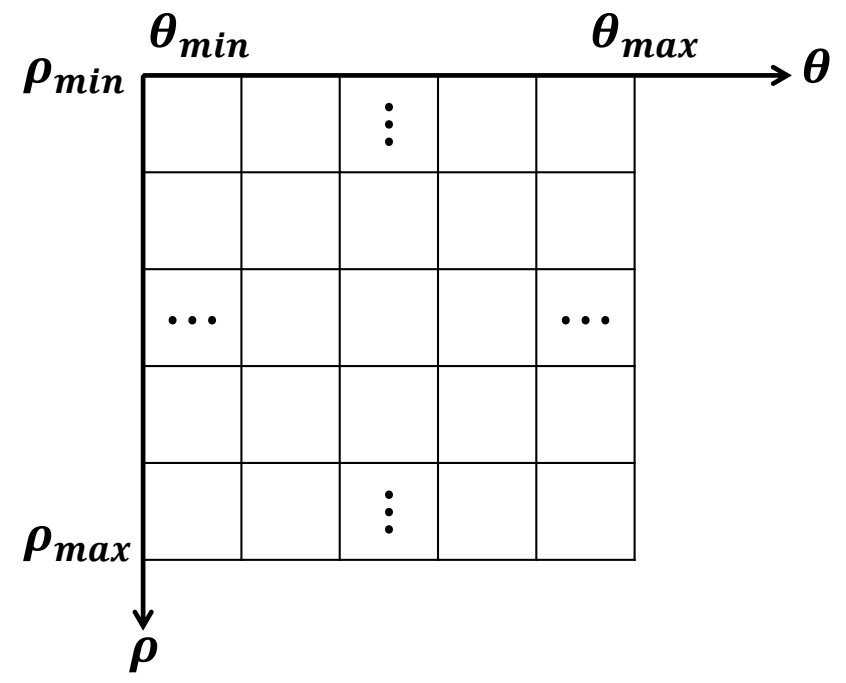

(b) Hough accumulator cells.

Figure 3.6: Hough Transform algorithm.

After detecting and representing line 1 to line 4 by the HT, the coordinates for the red points in Fig. 3.5 are estimated. These red points are used as the correspondence among the image sequences. Based on their coordinates, we derive the scale, linear translation, and rotation parameters to align all the image sequences. Fig 3.7(a) to 3.7(d) show the image registration results for the images in Fig. 3.3(a) to 3.3(d) respectively. 


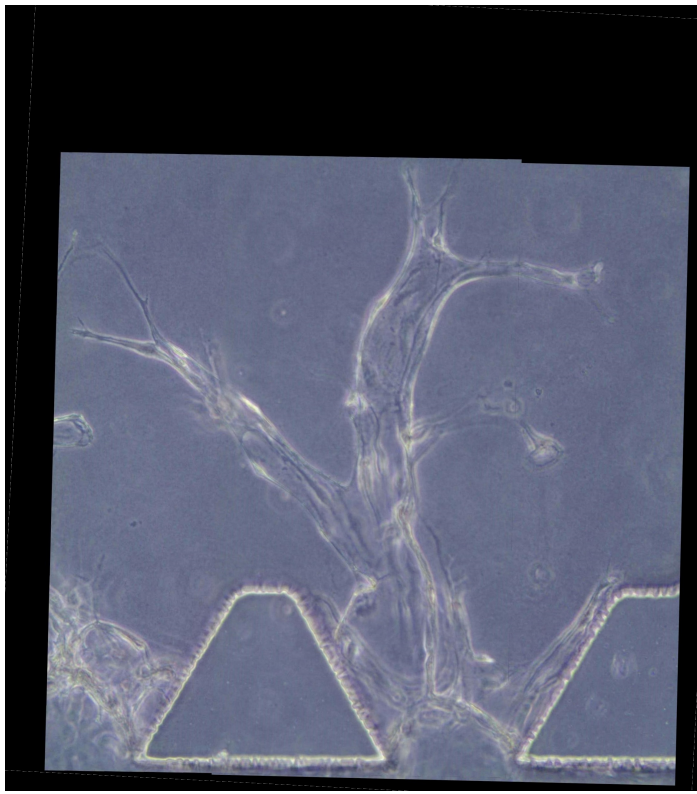

(a) Aligned image I.

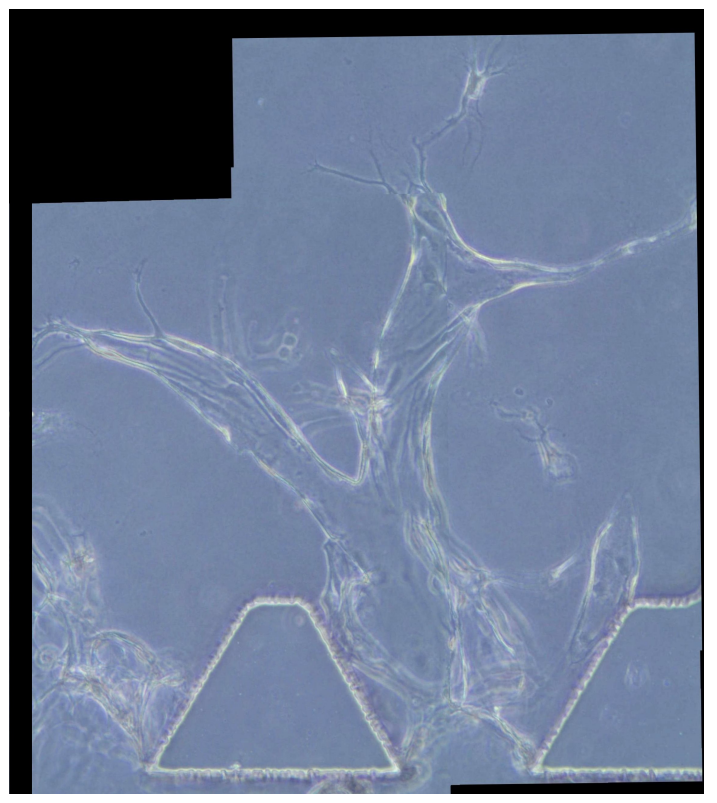

(c) Aligned image III

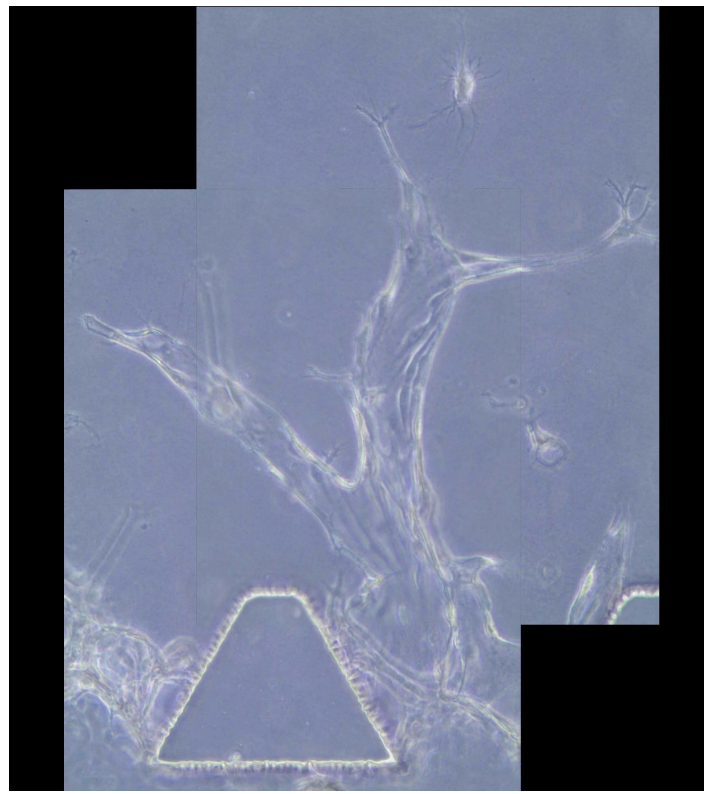

(b) Aligned image II.

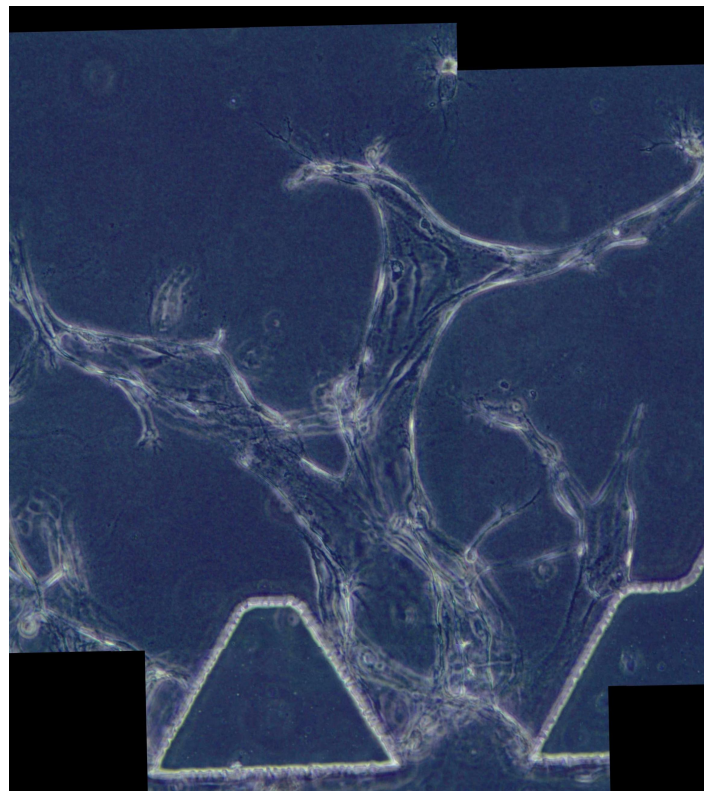

(d) Aligned image IV.

Figure 3.7: Image registration results for the examples in Fig. 3.3 showing the angiogenic vessels in a same sprouting region over 4 consecutive days (day 9 to day $12)$. 


\subsubsection{Vessel Segmentation}

Since the angiogenic vessels form in the collagen gel, we are mostly interested in the gel region. As illustrated in Fig. 3.8, the gel region is bounded by the post and the cell-gel interface. In order to remove the non-gel region, we first need to determine the boundaries of the gel region by detecting the posts and the cell-gel interface. The posts are detected and represented by the HT as discussed in image registration. As illustrated in Fig. 3.8, the cell-gel interface in the experimental images can be estimated by fitting a parabola. A parabola has the following expression:

$$
a_{1}\left(x-a_{p}+a_{3}\right)^{2}+a_{2}
$$

where $a_{p}$ is the intersection point of line 1 and line 4 obtained during image registration, and $a_{1}, a_{2}$, and $a_{3}$ are the coefficients of the parabola. Brute-force optimization is applied to determine the appropriate parabola equation to describe the monolayer [2]. Firstly, we provide a range of values for coefficients $a_{1}, a_{2}$, and $a_{3}$. By iterating over all possible combinations of coefficients, we find the image points that lie on each parabola and compute the line integral over the parabola. The coefficients of the parabola with maximum integral are selected as the optimal values for $a_{1}, a_{2}$, and $a_{3}$.

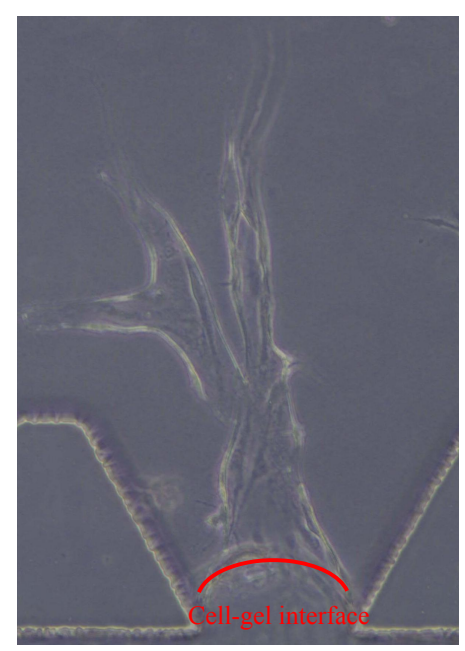

Figure 3.8: Example of the cell-gel interface in an experimental image. 


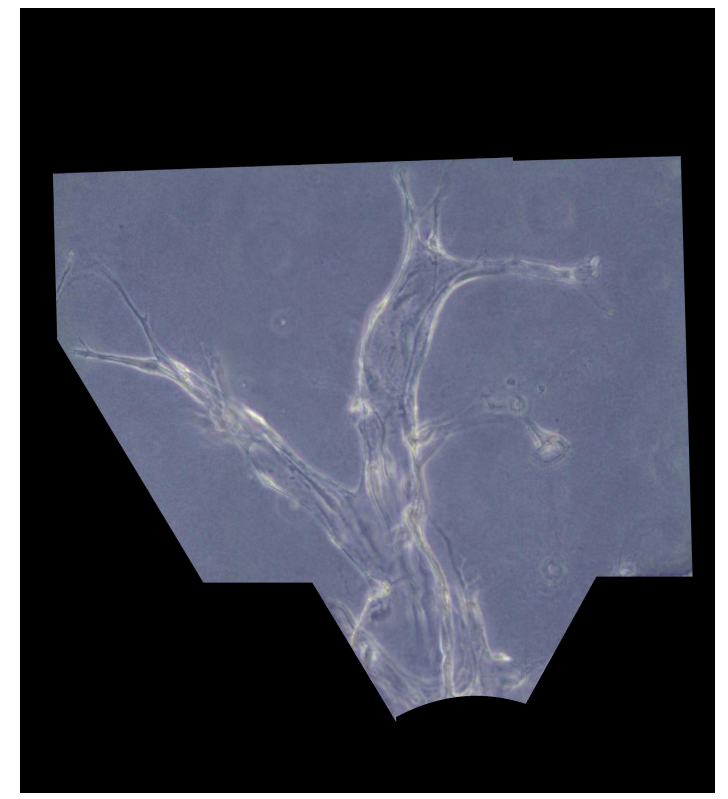

(a) Masked out non-gel region I.

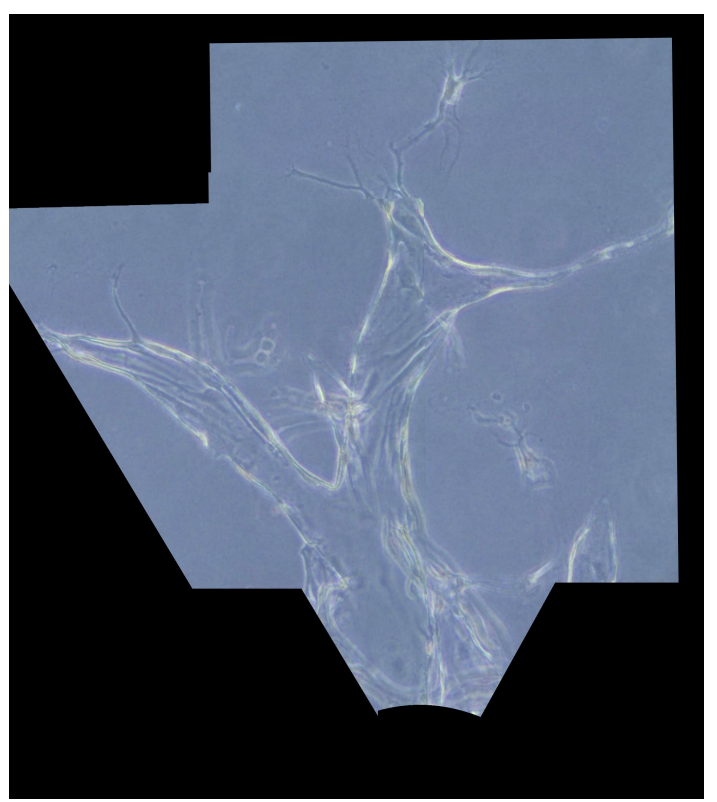

(c) Masked out non-gel region III.

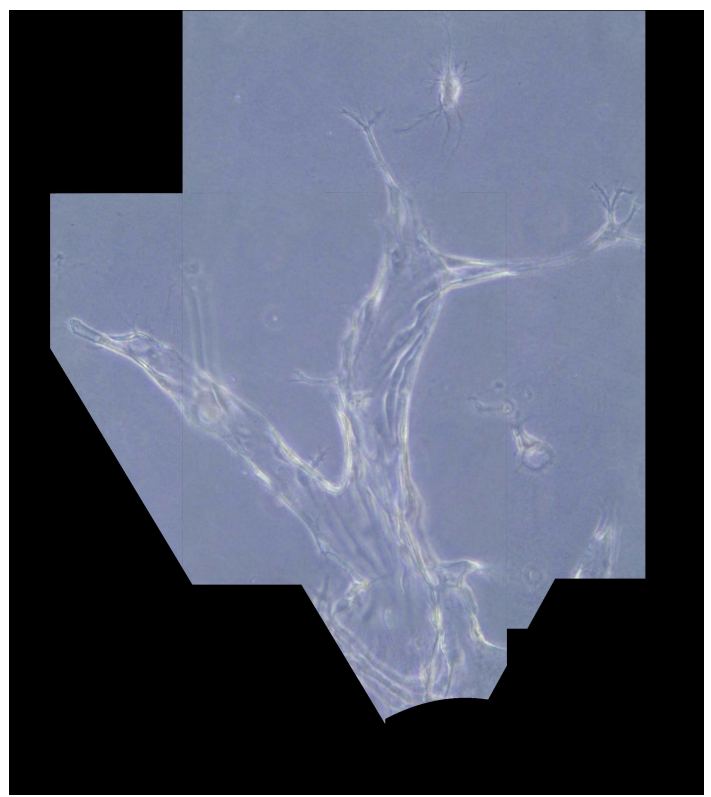

(b) Masked out non-gel region II.

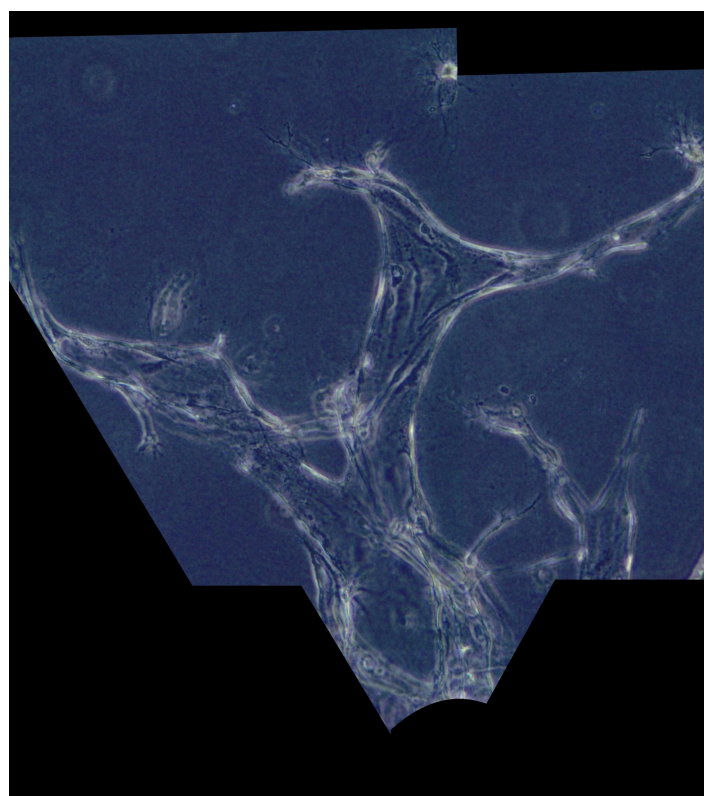

(d) Masked out non-gel region IV.

Figure 3.9: Images after masking out the non-gel region for the examples in Fig. 3.3: angiogenic vessels in a same sprouting region over 4 consecutive days (day 9 to day $12)$. 


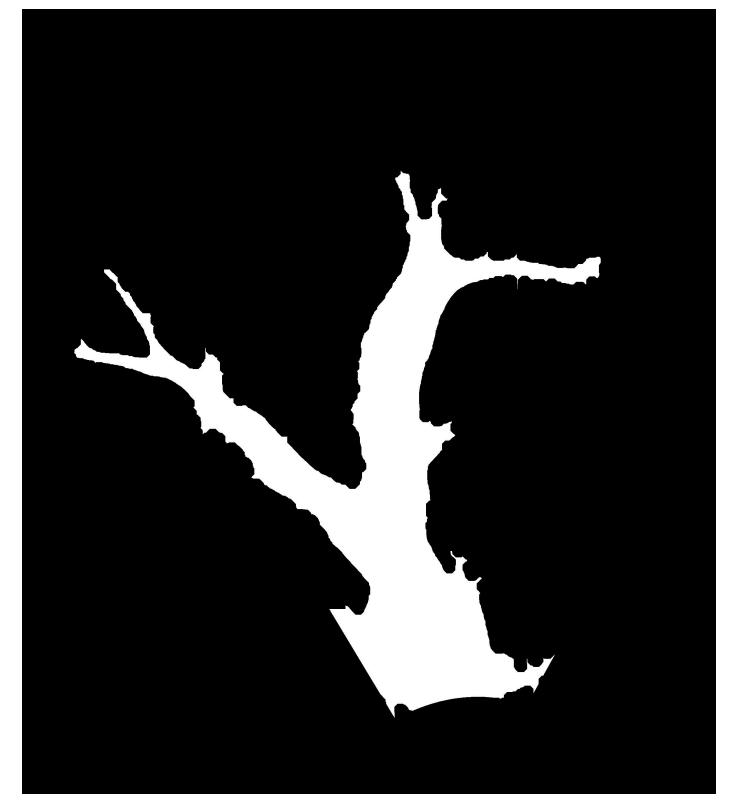

(a) Binary vessel shape I.

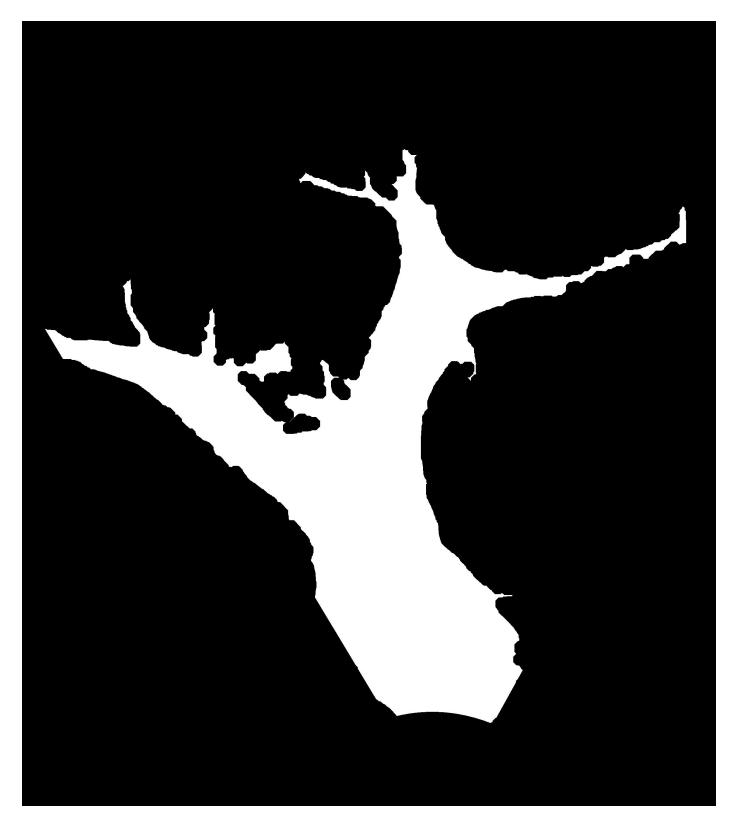

(c) Binary vessel shape III.

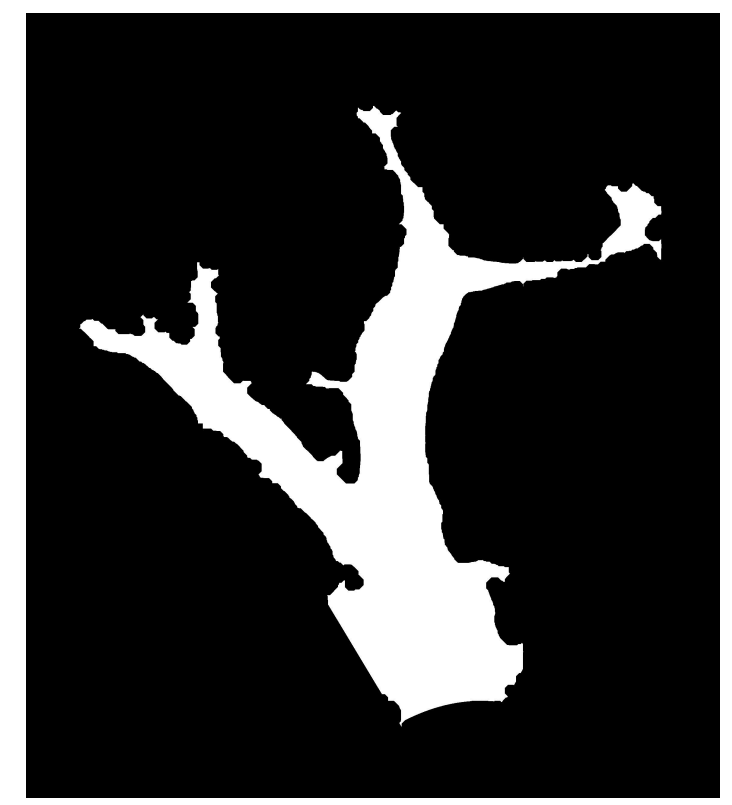

(b) Binary vessel shape II.

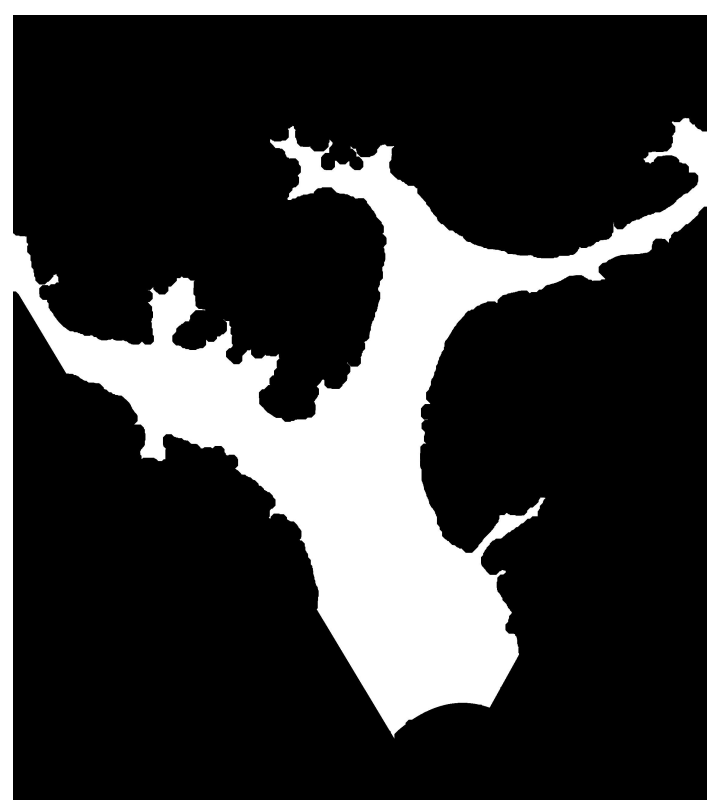

(d) Binary vessel shape IV.

Figure 3.10: Segmented binary shapes for the examples in Fig. 3.3: angiogenic vessels in the same sprouting region over 4 consecutive days (day 9 to day 12). 
We remove the non-gel region from the aligned phase contrast images (e.g. Fig. 3.7) by masking out the pixels in the identified post region and below the cell-gel interface, as shown in Fig. 3.9. These images are used in cell detection.

We then convert the processed experimental images in Fig. 3.9 into binary images with the global image threshold computed using Otsu's method [108]. We obtain the segmented vessel shape through a series of morphological image processing steps, including closing to fill the gaps in the boundaries, filling to fill the holes inside the vessel area, and area opening to remove the noise [88]. The segmented binary vessel shapes for the examples in Fig. 3.3(a) to 3.3(d) are shown in Fig. 3.10(a) to 3.10(d) respectively.

\subsubsection{Cell Detection}

We detect and label the EC candidates from the time-lapse phase contrast images through template matching. The flowchart is shown in Fig. 3.11. We first train a binary classifier offline based on the discriminative features extracted from the training templates (cell templates and non-cell templates). The resulting classifier then helps to distinguish the ECs from background in the test images through a sliding window manner.

We use the end-point confocal images, where we can easily recognize the cells, to select the cell templates. By aligning the phase contrast images with their corresponding confocal images, we can identify patches with cells and non-cells in the phase contrast images. These patches are used as our templates. Fig. 3.12 shows examples of cell and non-cell templates. The resulting training data consists of input images $\mathbf{X}$ and output labels $\mathbf{Y}$ :

$$
\begin{aligned}
& \mathbf{X}=\left[\mathbf{x}^{1}, \cdots, \mathbf{x}^{N}\right] \in \mathbb{R}^{m \times N}, \\
& \mathbf{Y}=\left[y^{1}, \cdots, y^{N}\right] \in\{-1,1\}^{N},
\end{aligned}
$$




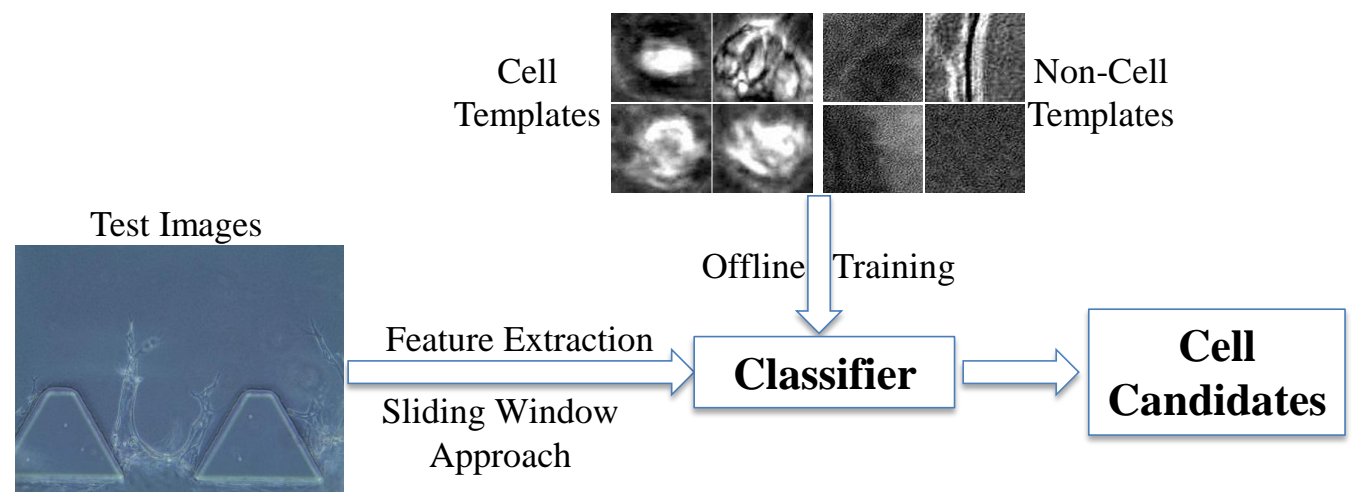

Figure 3.11: Template matching flowchart: The binary classifier is trained offline by means of discriminative features. After training, the classifier is applied to a sliding window that runs through the entire image, and distinguishes cells from background in each window.

where each $\mathbf{x}$ is an $m$-dimensional vector, each label $y$ is a binary value ( 1 for cell template and -1 for non-cell template), and superscripts $1, \cdots, N$ represent the template number. Specifically, we have 36 cell templates and 36 non-cell templates respectively $(N=72)$.

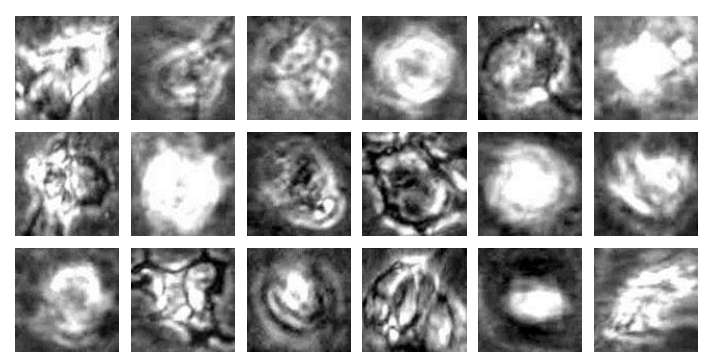

(a) Cell templates.
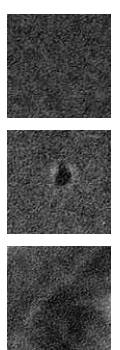
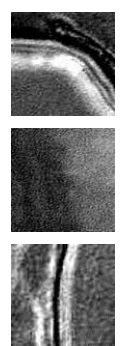
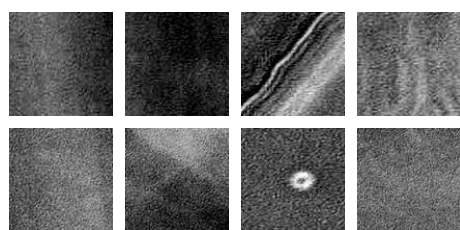

(a)
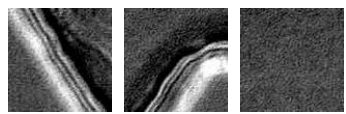

(b) Non-cell templates.

Figure 3.12: Examples of cell and non-cell templates from phase contrast images for offline training.

Principal Component Analysis (PCA) can be applied to obtain the discriminative features during offline training, followed by SVM for classification [109, 110]. However, our numerical results (see Section 3.3.2) indicate that the approaches mentioned above do not perform well for the problem at hand. Therefore, we decided to apply an alternative approach, i.e., PLSR, for both offline training and classification.

PLSR combines aspects of PCA and multi-linear regression. It is commonly used to 
extract a low-dimensional discriminative feature set to effectively predict the output data [111]. We apply PLSR for dimensionality reduction of the input training data and train a binary classifier to distinguish cell and non-cell templates, leveraging on the extracted discriminative features.

For our training set of input $\mathbf{X} \in \mathbb{R}^{m \times N}$ and output $\mathbf{Y} \in\{-1,1\}^{N}$, the PLSR algorithm for offline training consists of three steps.

The first step is to calculate the most correlated latent variable $\mathbf{z}_{1}$ by:

$$
\mathbf{z}_{1}=\mathbf{X}^{T} \mathbf{v}_{1} \in \mathbb{R}^{N \times 1}
$$

where $\mathbf{v}_{1}$ is the unit vector associated with the most correlated latent variable $\mathbf{z}_{1}$. $\mathbf{v}_{1}$ is calculated by maximizing the correlation $J$ between the latent variable and the output Y (see Fig. 3.13):

$$
\mathbf{v}_{1}=\arg \max _{\mathbf{v}} J(\mathbf{v})=\frac{\mathbf{X} \mathbf{Y}^{T}}{\left|\mathbf{X} \mathbf{Y}^{T}\right|}
$$

The second step is to predict the output $\mathbf{Y}$ from this most correlated latent variable

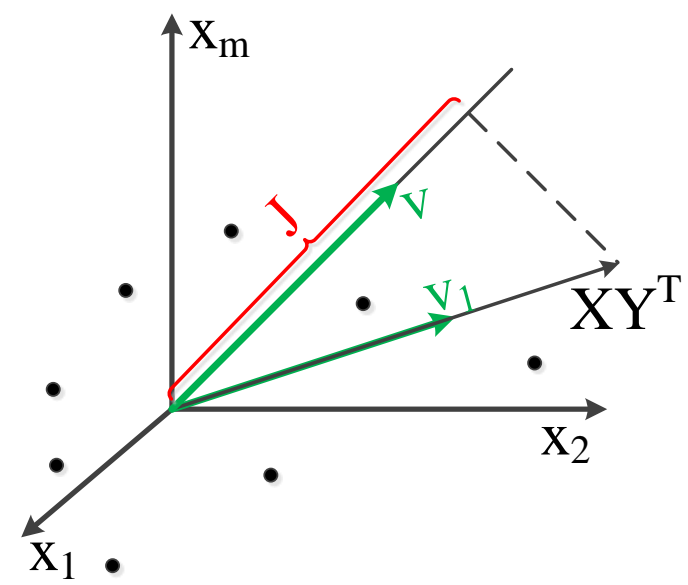

Figure 3.13: Diagram of partial least square regression. 
$\mathbf{z}_{1}$ by linear prediction. For one template $i$, the predicted output $\hat{y}^{i}$ is calculated by:

$$
\hat{y}^{i}=c_{1} \mathbf{z}_{1}^{i}
$$

where $c_{1}$ is the scalar coefficient correlated with $\mathbf{z}_{1}$. It is calculated by minimizing the mean square error:

$$
c_{1}=\arg \min _{c} \sum_{i=1}^{N}\left(y^{i}-c \mathbf{z}_{1}^{i}\right)=\frac{\mathbf{Y} \mathbf{z}_{1}}{\mathbf{z}_{\mathbf{1}}^{\mathbf{T}} \mathbf{z}_{\mathbf{1}}} .
$$

The prediction of output based on the single latent variable $\mathbf{z}_{1}$ may be limited in accuracy since $\mathbf{X}$ and $\mathbf{Y}$ may have correlations in other directions. Thus, the last step is to examine the correlation between the input and output residues. The input residue $\mathbf{X}^{\prime}$ and output residue $\mathbf{Y}^{\prime}$ are estimated by:

$$
\begin{aligned}
& \mathbf{X}^{\prime}=\mathbf{X}-\mathbf{X} \frac{\mathbf{z}_{1} \mathbf{z}_{1}^{\mathbf{T}}}{\mathbf{z}_{1}^{\mathbf{T}} \mathbf{z}_{\mathbf{1}}}, \\
& \mathbf{Y}^{\prime}=\mathbf{Y}-c_{1} \mathbf{z}_{1}^{T} .
\end{aligned}
$$

We expect that the accuracy would increase by introducing another latent variable $\mathbf{z}_{2}$, which is responsible of predicting the residue that $\mathbf{z}_{1}$ could not predict. The second latent variable $\mathbf{z}_{2}$ and its correlation coefficient $c_{2}$ can be determined by repeating the first two steps but replacing the input $\mathbf{X}$ and output $\mathbf{Y}$ with the new residues $\mathbf{X}^{\prime}$ and $\mathbf{Y}^{\prime}$ respectively.

We iterate the above steps until the correlation between the input and output residues diminishes to obtain a series of latent variables $\mathbf{z}_{1}, \cdots, \mathbf{z}_{m^{*}}$ and their corresponding coefficients $c_{1}, \cdots, c_{m^{*}}$. For one template $i$, the output $\hat{y}^{i}$ is predicted by:

$$
\hat{y}^{i}=c_{1} \mathbf{z}_{1}^{i}+\cdots+c_{m^{*}} \mathbf{z}_{m^{*}}^{i}
$$


where $m^{*}$ is the optimal number of PLS components.

In order to determine the optimal number of PLS components $m^{*}$, we monitor the percentage variance explained in the output variables. Our goal is to achieve $95 \%$ of the variance explained in the output variables with the smallest possible number of PLS components. From Fig. 3.14, which shows the relationship between the percent of variance explained and the number of PLS components, $m^{*}=4$ is selected, where $96 \%$ of the variance explained is achieved in the output variables.

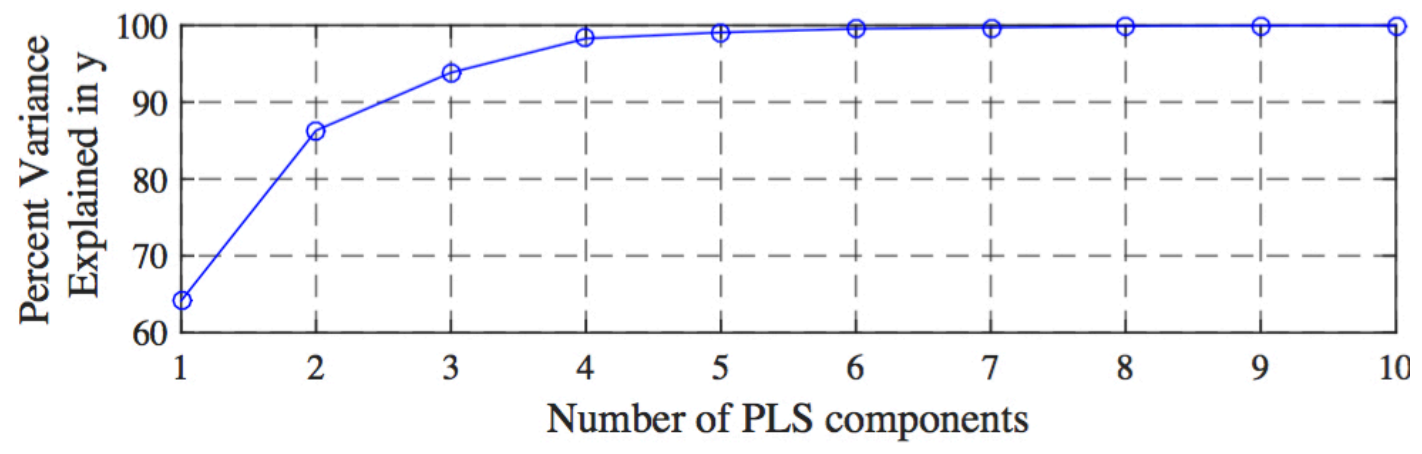

Figure 3.14: Percent of variance explained v.s. the number of PLS components.

The PLSR approach for cell detection is summarized in Algorithm 1. Following this method, we extract the EC candidates in each frame in an automated manner. As we know that ECs migrate within the angiogenic vessels, we eliminate the EC candidates that are located outside the vessel area.

\subsubsection{Multiple Hypothesis Kalman Filtering}

We combine backward Kalman filtering with MHT to associate and track the detected EC candidates within the angiogenic vessels over time. Instead of initializing the tracks with the detected EC candidates from the images at the first time point, we initialize our tracks with the confocal images at the end time point since they provide more accurate EC locations and all EC proliferations have occurred. Furthermore, we incorporate biological knowledge to facilitate cell association. For 


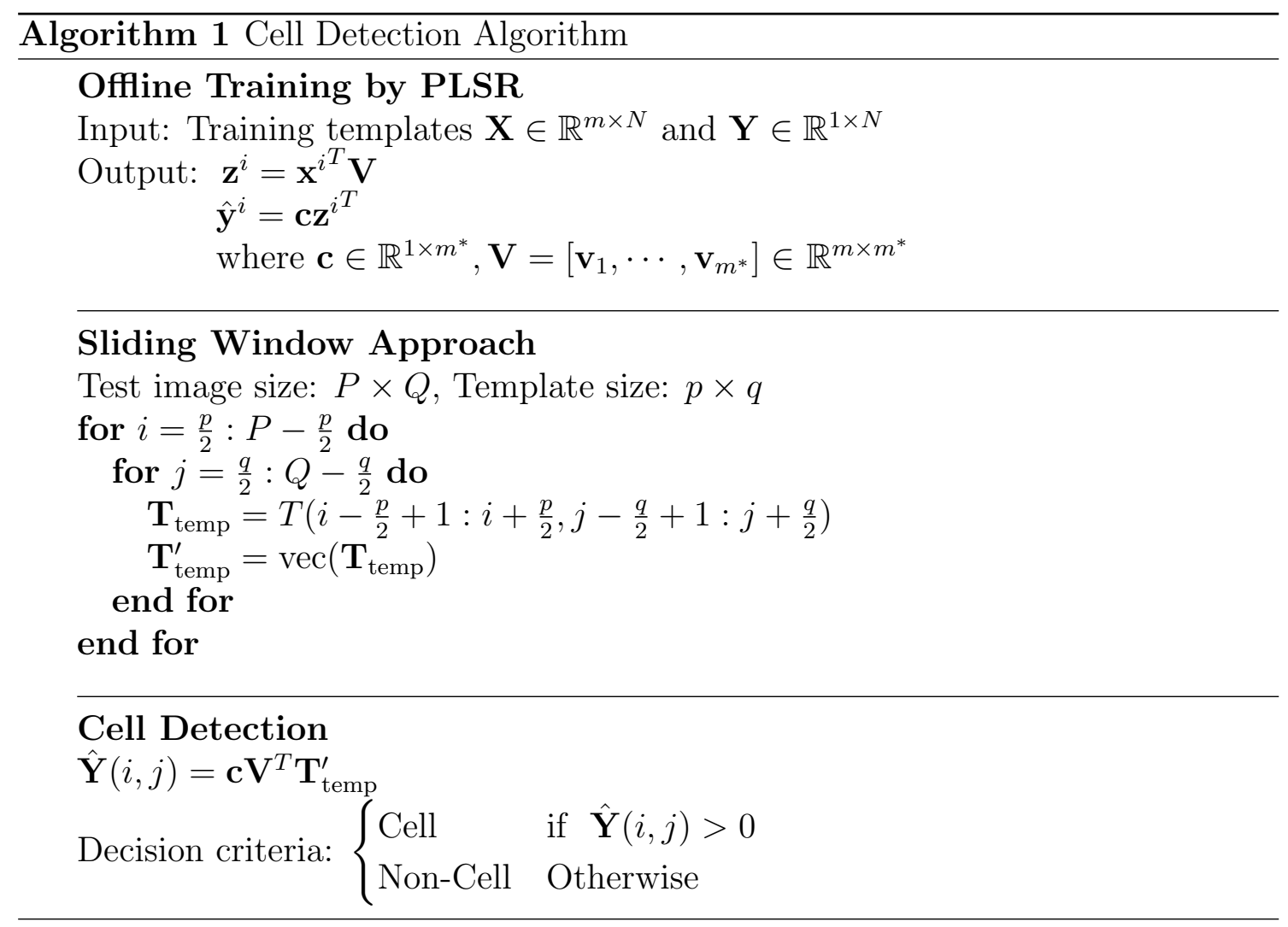

instance, since ECs cannot take over each other due to tight cell junctions, we eliminate the track where overtaking happens. Moreover, we assume one EC can at most split once within one day because the generation time of the HMVECs is 15 to 48 hours.

We denote each individual cell state by $\mathbf{x}_{k}=\left[x_{k}, y_{k}, \dot{x}_{k}, \dot{y}_{k}\right]^{T}$, where $x_{k}$ and $y_{k}$ are the estimated cell centroid positions in the $x$-axis and $y$-axis, and $\dot{x}_{k}$ and $\dot{y}_{k}$ are their corresponding velocities. The system covariance at time $k$ is denoted by $\mathbf{P}_{k}$. The observation $\mathbf{z}_{k}=\left[x_{k}, y_{k}\right]^{T}$ is the cell position obtained in the cell detection process.

\subsubsection{Backward Kalman Filter}

The backward Kalman filter consists of two steps: backward prediction and update. The prediction equations for the prior state estimate $\hat{\mathbf{x}}_{k-1 \mid k}$ and corresponding sys- 
tem covariance estimate $\mathbf{P}_{k-1 \mid k}$ are:

$$
\begin{aligned}
\hat{\mathbf{x}}_{k-1 \mid k} & =\mathbf{F} \hat{\mathbf{x}}_{k \mid k}, \\
\mathbf{P}_{k-1 \mid k} & =\mathbf{F P}_{k \mid k} \mathbf{F}^{T}+\mathbf{Q},
\end{aligned}
$$

where $\mathbf{F}$ is the state transition model, and $\mathbf{Q}$ is the covariance matrix of the process noise. We assume a constant velocity model, hence we have

$$
\mathbf{F}=\left[\begin{array}{cccc}
1 & 0 & \Delta t & 0 \\
0 & 1 & 0 & \Delta t \\
0 & 0 & 1 & 0 \\
0 & 0 & 0 & 1
\end{array}\right]
$$

where $\Delta t$ is the time interval. Since we track the EC candidates backward, $\Delta t=$ -1 day is used.

The update equations for the cell state and covariance matrix are:

$$
\begin{aligned}
\hat{\mathbf{x}}_{k-1 \mid k-1} & =\hat{\mathbf{x}}_{k-1 \mid k}+\mathbf{K}_{k-1} \tilde{\mathbf{y}}_{k-1}, \\
\mathbf{P}_{k-1 \mid k-1} & =\left(\mathbf{I}-\mathbf{K}_{k-1} \mathbf{H}\right) \mathbf{P}_{k-1 \mid k},
\end{aligned}
$$

where $\tilde{\mathbf{y}}_{k-1}$ is the innovation or the residual error between the predicted and observed estimate, $\mathbf{S}_{k-1}$ is the innovation covariance, and $\mathbf{K}_{k-1}$ is the optimal Kalman gain matrix, yielding the minimum mean square error. $\mathbf{H}$ is the observation model that maps the state space into the observation space:

$$
\mathbf{H}=\left[\begin{array}{llll}
1 & 0 & 0 & 0 \\
0 & 1 & 0 & 0
\end{array}\right]
$$

$\tilde{\mathbf{y}}_{k-1}, \mathbf{S}_{k-1}$ and $\mathbf{K}_{k-1}$ are calculated as follows:

$$
\tilde{\mathbf{y}}_{k-1}=\mathbf{z}_{k-1}-\mathbf{H} \hat{\mathbf{x}}_{k-1 \mid k}
$$




$$
\begin{aligned}
\mathbf{S}_{k-1} & =\mathbf{H P}_{k-1 \mid k} \mathbf{H}^{T}+\mathbf{R} \\
\mathbf{K}_{k-1} & =\mathbf{P}_{k-1 \mid k} \mathbf{H}^{T}\left(\mathbf{H P}_{k-1 \mid k} \mathbf{H}^{T}+\mathbf{R}\right)^{-1}
\end{aligned}
$$

where $\mathbf{R}$ is the covariance of the observation noise.

\subsubsection{Backward Kalman Filter with Validation Gating for Cell Asso- ciation}

The backward Kalman filter with validation gating is a popular approach to update the cell state during cell association. In each image, we extract multiple cells, which will be associated to multiple tracks. To associate an observation to a particular track, we employ a validation gate as an association threshold of whether to accept or reject an observation-to-target association. Observations which fall within the validation gate will be updated.

As shown in Fig. 3.15, the blue and red ellipse are the validation gate for cell 1 and

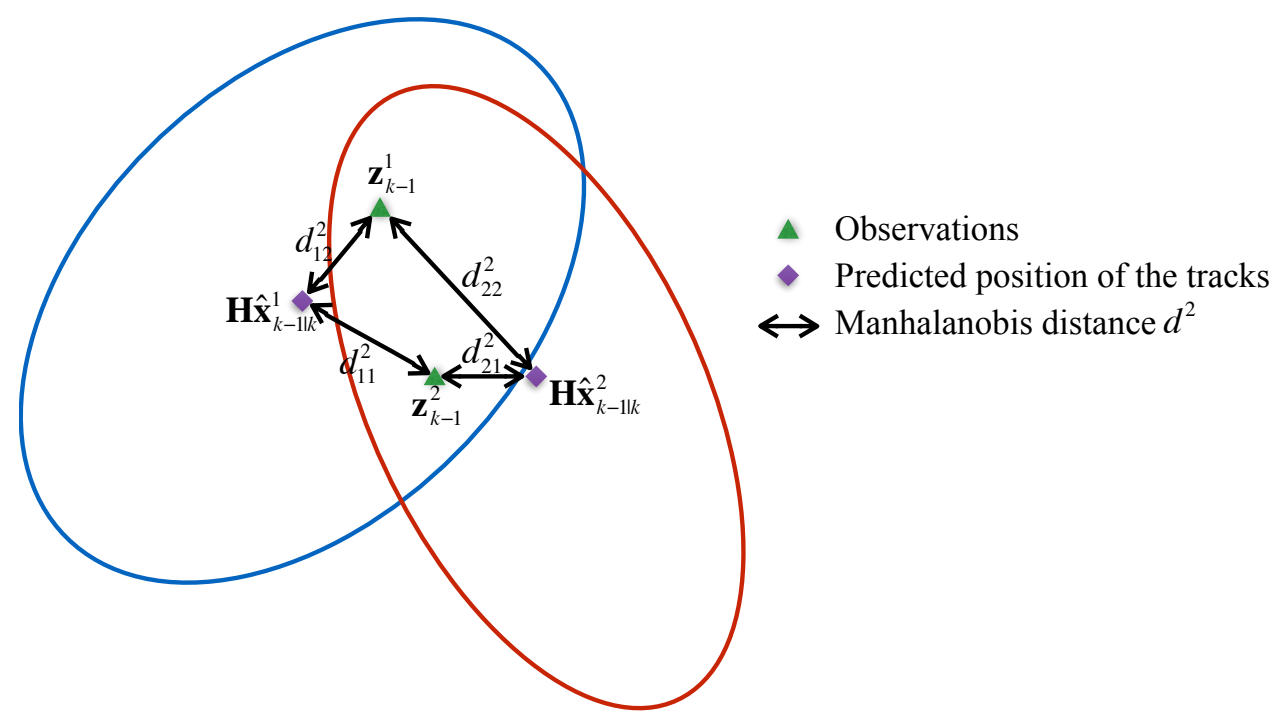

Figure 3.15: Backward Kalman filter with validation gating for cell association: blue and red ellipses are the validation gate for track 1 and 2 respectively; track 1 is associated with observation 1 and track 2 is associated with observation 2 in this plot. 
cell 2 respectively. Specifically, we compute the Mahalanobis distance (a measure of the distance between a point and a distribution) between each observation to each state estimate as:

$$
d_{i j}^{2}=\tilde{\mathbf{y}}_{i j, k-1}^{T} \mathbf{S}_{i j, k-1}^{-1} \tilde{\mathbf{y}}_{i j, k-1}
$$

where $\tilde{\mathbf{y}}_{i j, k-1}$ is the innovation between the back-predicted track $i$ and the observation $j$, and $\mathbf{S}_{i j, k-1}$ is the corresponding innovation covariance. The observation whose Mahalanobis distance to a track is smaller than the validation gate is more likely belonging to this track. If there are no observations within this gate, no observation updates to this track will occur. When there are multiple observations in a validation gate, the estimate is updated with only the best-matched observation (smallest Mahalanobis distance). For instance, observation 1 is associated with track 1 and observation 2 is associated with track 2 in Fig. 3.15.

\subsubsection{Multiple Hypothesis Kalman Filtering for Cell Association}

An alternative approach for the multiple observations problem is to apply MHT, which allows for delay decision till sufficient information is available. We combine backward Kalman filter with MHT to associate the detected EC candidates, as illustrated in Fig. 3.16. During cell association, backward Kalman filter with validation gating makes decisions every time step. However, in multiple hypothesis Kalman filtering, all the possible hypotheses in step $k$ are maintained and considered independently to generate new hypotheses in the next time step. The cell associations are obtained by selecting the most probable hypotheses.

At the end time point of the angiogenic experiments, which is the initial time point of the backward Kalman filter, we create one track for each cell. Each cell state and covariance estimate at time $k$ are back-propagated to time $k-1$ using (3.13) and (3.14). If there are $N_{k-1}$ observations which fall within the validation gate, 


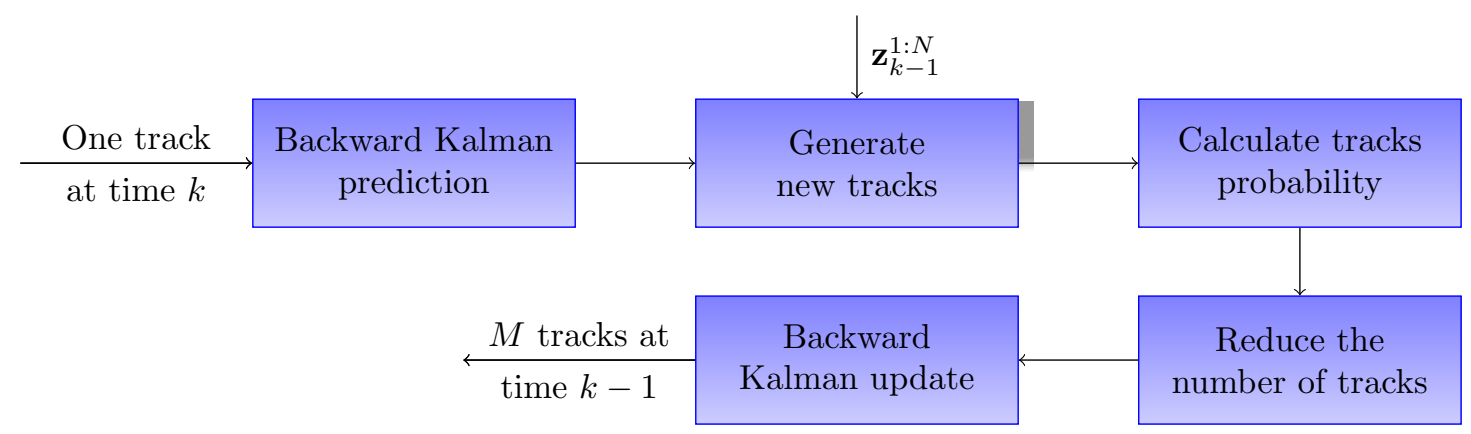

Figure 3.16: Flowchart of multiple hypothesis Kalman filtering. Cell state, system covariance, and probabilities of all the tracks are recursively updated over time, corresponding to multiple hypotheses. The cell associations are obtained by selecting the most probable hypotheses.

MHT associates all the $N_{k-1}$ observations to the same track by generating $N_{k-1}$ new branches to the track. Fig. 3.17 provides an example of the multiple hypothesis Kalman filtering process for one cell in two successive time steps. Within the blue ellipse, the purple diamond represents the predicted position of one track at time $k-1$. The green triangles indicate the observations that may be associated with this track with probability $p_{1}, p_{2}$, and $p_{3}$ respectively. The red pentagon depicts the hypothesis (with probability $p_{0}$ ) that the track is coming from the cell-gel interface. All the possible hypotheses in step $k-1$ are maintained and considered independently to generate new hypotheses in the next time step, as indicated by the red ellipses in Fig. 3.17. Since the number of branches for each track increases exponentially, in order to keep the computation under control, we maintain $M$ branches for each time by considering biological knowledge and pruning the tracks with low probability.

Let us consider cell $i$ at time $k$. This cell might be due to the migration or proliferation of an existing cell labeled as $j$ at time $k-1$. It is also possible that this cell is in the monolayer or out of focus (not in the focal plane) at time $k-1$. The corresponding probabilities are calculated in different ways.

We employ the Mahalanobis distance to calculate the probability that track $i$ stems from the migration or proliferation of an existing cell $j$. If the distance is smaller 


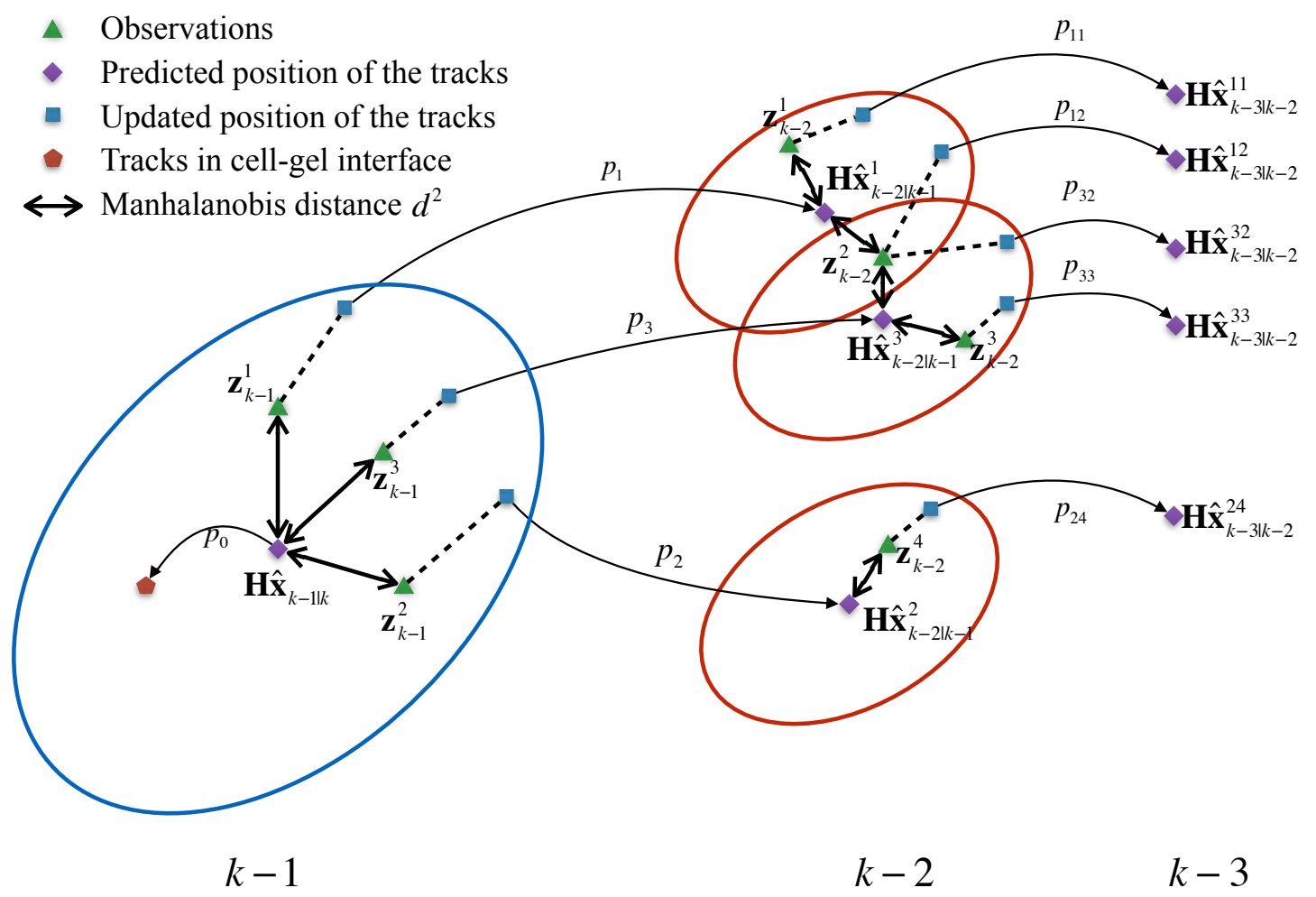

Figure 3.17: Multiple hypothesis Kalman filtering for one cell in two successive time steps.

than the threshold gate value, the probability that the track $i$ is associated with observation $j$ is calculated as:

$$
p_{i}\left(\mathbf{z}_{k-1}^{j}\right) \propto\left|2 \pi \mathbf{S}_{i j, k-1}\right|^{-\frac{1}{2}} e^{-\frac{1}{2} d_{i j}^{2}}
$$

where $d_{i j}^{2}$ is the Mahalanobis distance between the back-predicted track $i$ and the observation $j$, and $\mathbf{S}_{i j, k-1}$ is the innovation covariance estimated by (3.20).

We assume that the cells nearer to the cell-gel interface (larger $y$-position) are more likely to be new tracks. The probability that track $i$ is due to migration from the cell-gel interface is computed as:

$$
p_{i}(\mathrm{M}) \propto \frac{1}{\left(b_{1}-y_{k-1 \mid k}\right)^{b_{2}}},
$$

where $b_{1}$ and $b_{2}$ are empirical parameters tuned from our experimental data, and 
$y_{k-1 \mid k}$ is the cell position in $y$-direction from backward Kalman prediction.

We also incorporate biological knowledge to reduce the number of the new tracks in each step. The generation time is the average time of one cell cycle. Since the generation time of the HMVECs is 15 to 48 hours, we assume that one cell can at most split once within one day. In other words, we allow at most two cells at time $k$ to be associated with one same observation at time $k-1$ during merging. In the angiogenic vessels, cells cannot overtake each other due to the existence of tight cell junctions, so we eliminate the tracks where overtaking happens during association. We discard the tracks with low probability in order to keep the number of tracks under control.

We keep $M$ branches and obtain their corresponding cell state and system covariance by a backward Kalman update for each time step. Typically, $M=4$ is selected since the remaining branches have negligible probabilities. Each of these new tracks is considered independently and used to generate new predictions for next time step. We repeat this tracking process until $k=1$, and obtain $N_{0} M^{t-1}$ hypotheses in total, where $N_{0}$ is the number of the cells obtained from the end-point confocal images and $t$ is the total time steps. Lastly, the tracks are selected as the most probable hypotheses.

\subsection{Results and Discussion}

We apply the proposed AMCTS to track the ECs migration within angiogenic vessels from the time-lapse phase contrast images. In Section 3.3.1, we show the results from the preprocessing step. In Section 3.3.2, we present the cell detection results. We elaborate on the cell tracking results and provide the cell lineage plots in Section 3.3.3. 


\subsubsection{Preprocessing Results}

We provide two examples to demonstrate the results from our proposed preprocessing step in Fig. 3.18. Fig. 3.18(a) and 3.18(e) show the original end-point phase contrast images. Fig. 3.18(b) and 3.18(f) are their corresponding confocal images, where the blue blobs are the cell nuclei stained with Hoechst, and the green structures are the actin stained with Phalloidin 488. We can see that the size and position of the angiogenic sprouts are different in the phase contrast images and confocal images. After alignment, we overlay the end-point phase contrast images and their corresponding end-point confocal images to better illustrate the image registration results. As shown in Fig. 3.18(c) and 3.18(g), the resized phase contrast images are shown in the background and the cell nuclei stained in the confocal images are marked as green blobs for clearer identification. The angiogenic structures are well-aligned in Fig. 3.18(c) and 3.18(g), suggesting our image registration algorithm is accurate. Image registration transforms the experimental image sequences into one coordinate system, which is critical for investigating ECs migration and vessel formation. Fig. 3.18(d) and 3.18(h) show the results of vessel segmentation.

\subsubsection{Cell Detection Results}

We use the same examples as in Section 3.3.1 (see Fig. 3.18) to illustrate our cell detection results, as shown in Fig. 3.19.

Fig. 3.19(a) and 3.19(d) show the results from the proposed cell detection (PLSR) algorithm. We also applied two other cell detection approaches for comparison. For the first method, we trained an SVM classifier based on the original highdimensional template data to distinguish the cells from the background. The results are shown in Fig. 3.19(b) and 3.19(e). For the second approach, we used PCA to reduce the dimension of the original template data followed by training an SVM classifier based on the dimension-reduced features. The detected ECs are illustrated 


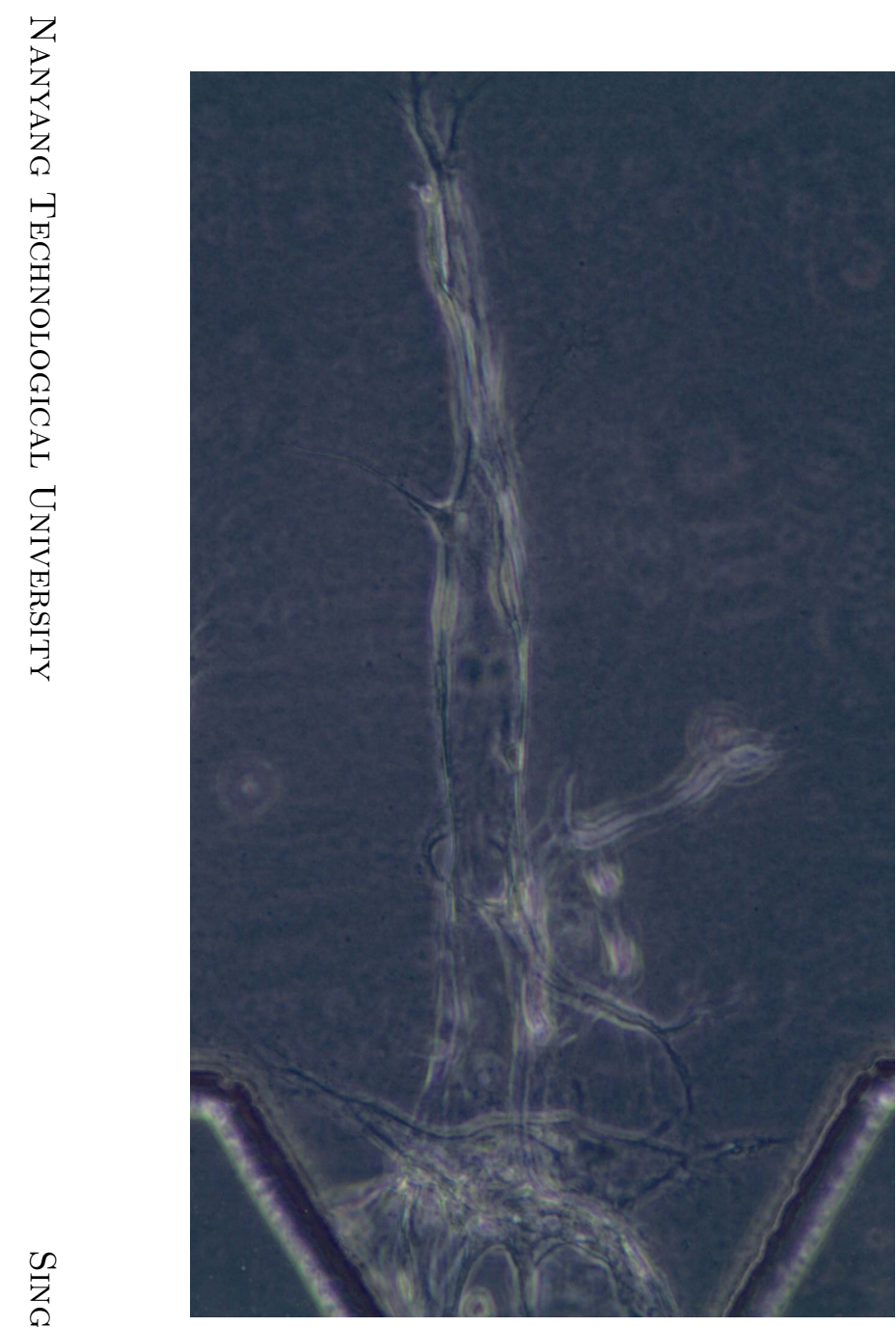

(a) Phase contrast image I.

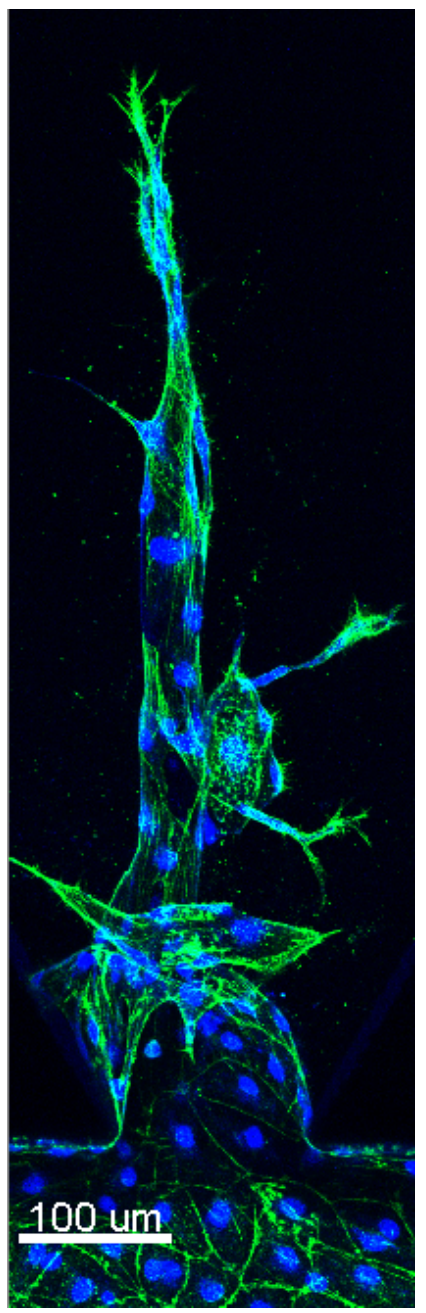

(b) Confocal image I.

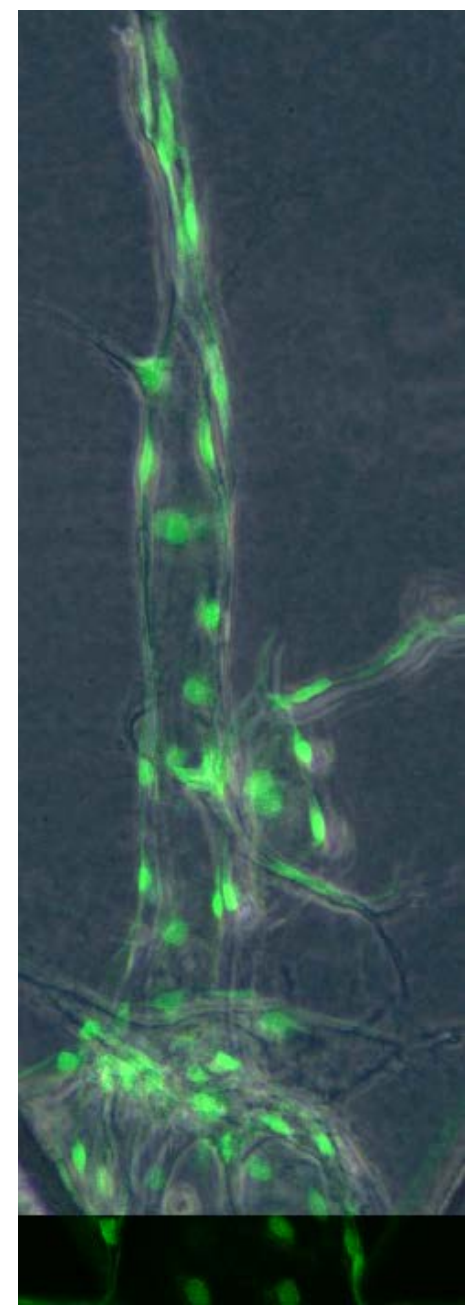

(c) Aligned image I.

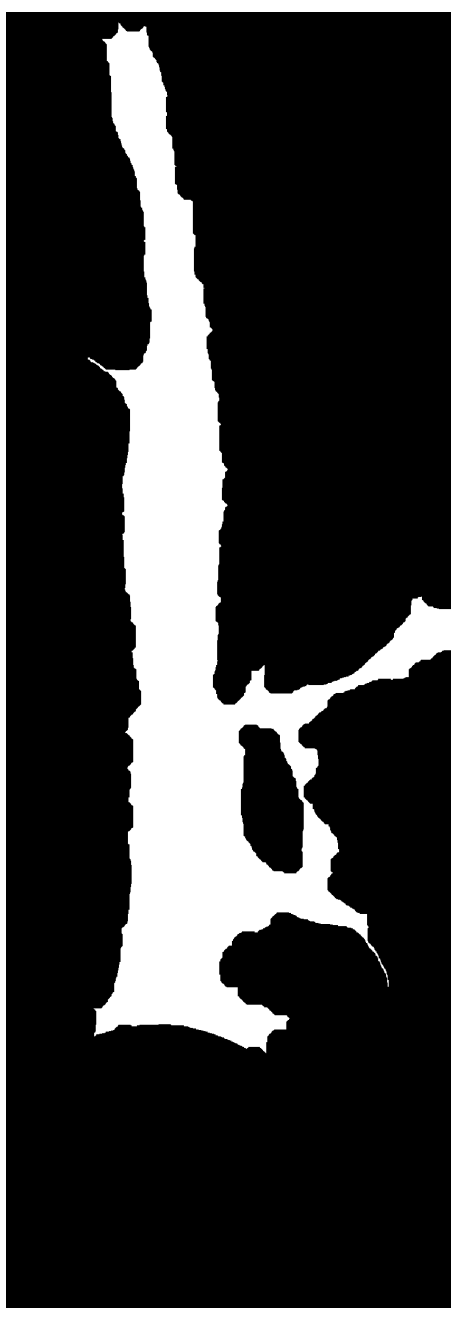

(d) Binary vessel shape I. 


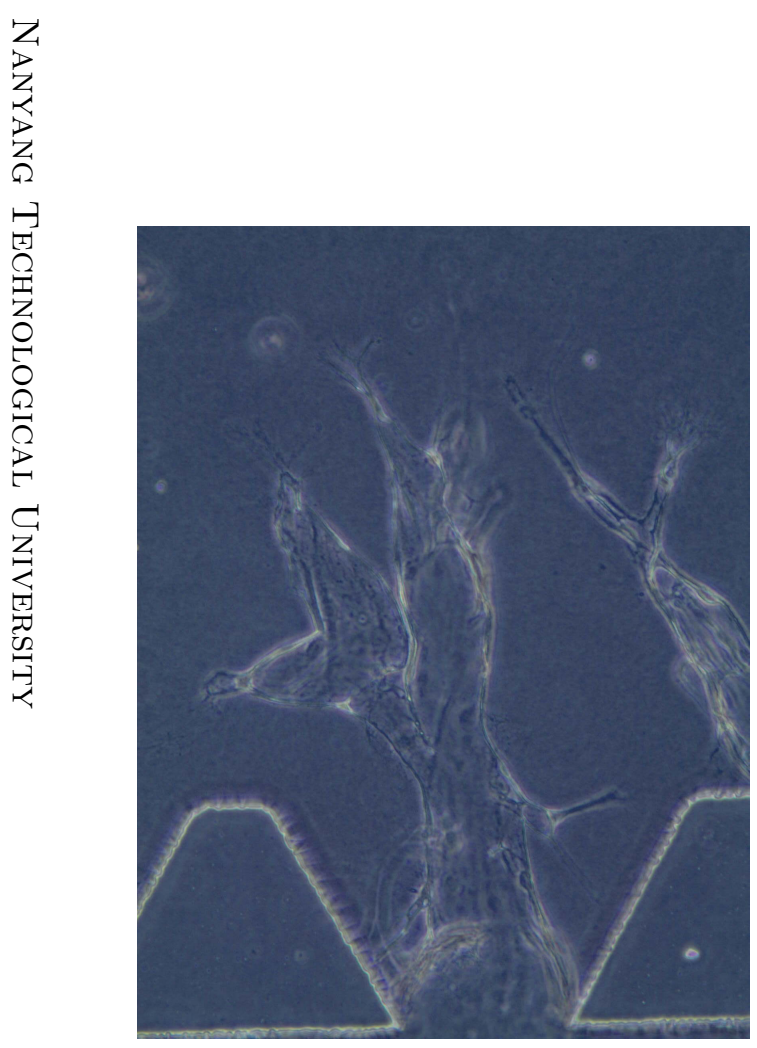

(e) Phase contrast image II.

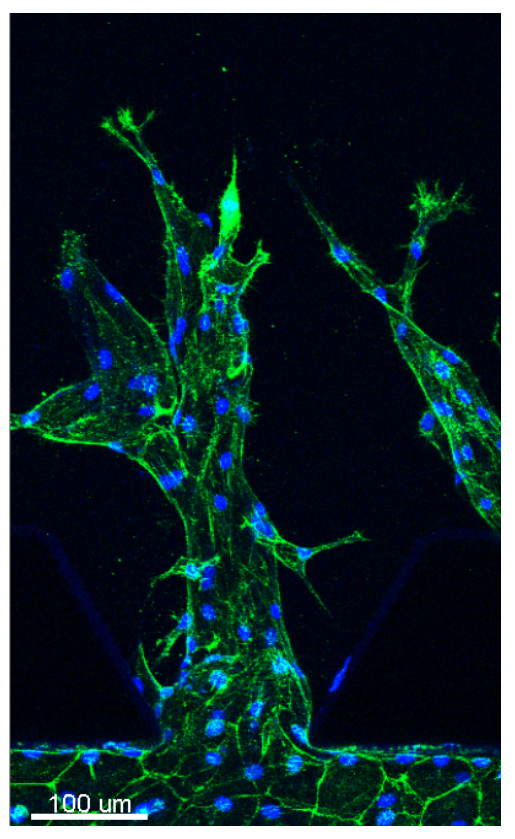

(f) Confocal image II.

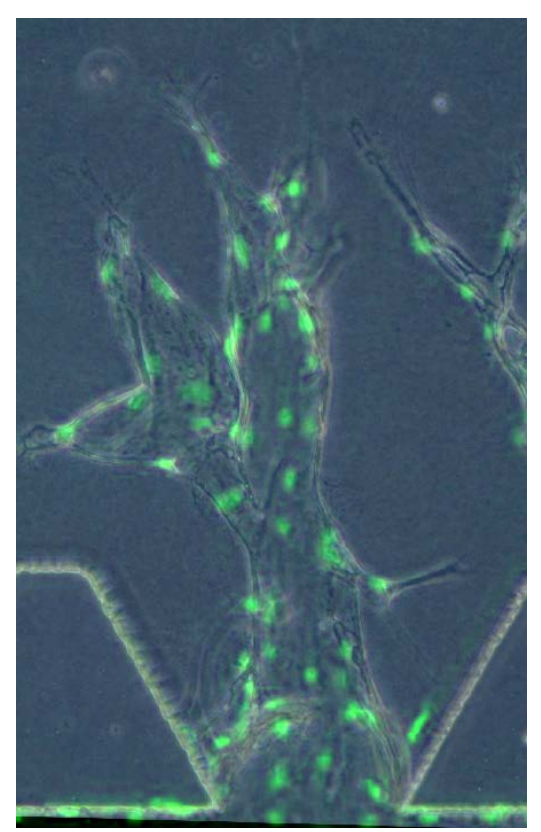

(g) Aligned image II.

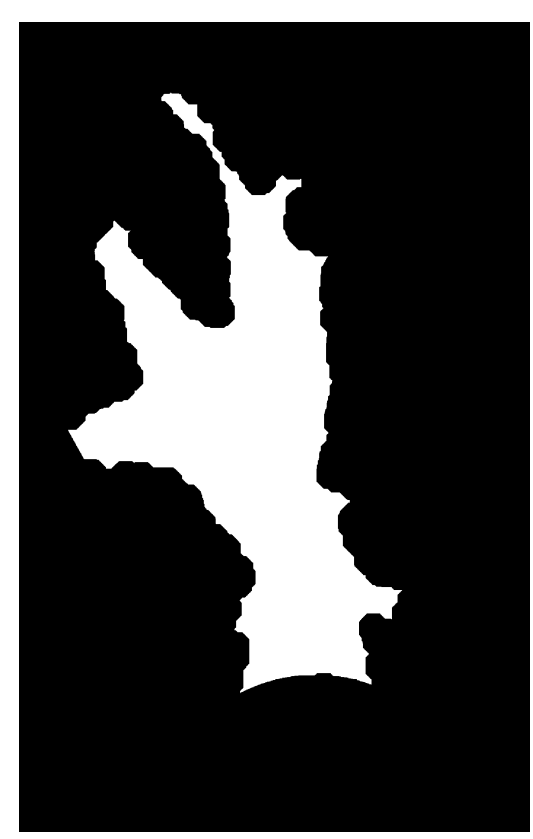

(h) Binary vessel shape II.

Figure 3.18: Illustration of preprocessing results: (a) and (e) are the end-point phase contrast images; (b) and (f) are their corresponding confocal images; (c) and (g) show the co-registered confocal and phase contrast images (the nuclei color is changed

from blue to green just for clearer visualization); (d) and (h) are the binary vessel shapes from vessel segmentation. 
in Fig. 3.19(c) and 3.19(f). The red stars (*) label the locations of the detected EC nuclei for all the images in Fig. 3.19. It can be seen that cell detection using PLSR outshines the two other approaches.

In order to evaluate the performance of all these approaches quantitatively, precision and recall measures are calculated. Precision is the fraction of detected cells that are actual cells, and recall is the fraction of the actual cells that are detected:

$$
\begin{aligned}
\text { Precision } & =\frac{\mathrm{TP}}{\mathrm{TP}+\mathrm{FP}}, \\
\text { Recall } & =\frac{\mathrm{TP}}{\mathrm{TP}+\mathrm{FN}},
\end{aligned}
$$

where TP, FP and FN are defined in the $2 \times 2$ confusion matrix in Table 3.1 [3]. This confusion matrix describes the four possible outcomes for a given binary classifier and a set of instances.

Table 3.1: Confusion matrix for a binary classifier [3].

\begin{tabular}{|c|c|c|}
\hline & Actual Positive & Actual Negative \\
\hline Assigned Positive & True Positive (TP) & False Positive (FP) \\
\hline Assigned Negative & False Negative (FN) & True Negative (TN) \\
\hline
\end{tabular}

As discussed, end-point confocal images provide accurate cell locations. We use the end-point phase contrast images to validate the cell detection results by matching the estimated cell locations with their corresponding confocal images. We analyzed 18 end-point phase contrast images in this chapter to estimate the cell detection performance.

Since the phase contrast image is a $2 \mathrm{D}$ slice and the confocal image is a $3 \mathrm{D}$ stack with multiple slices, we choose the ground truth in two ways. For the first case, the actual positives and negatives are extracted from all the slices of confocal images. However, since our phase contrast image is in 2D, we use only one slice of the confocal image to provide the ground truth for the second case. The slide, which has a similar focal plane as the $2 \mathrm{D}$ phase contrast image, is chosen from the $3 \mathrm{D}$ 


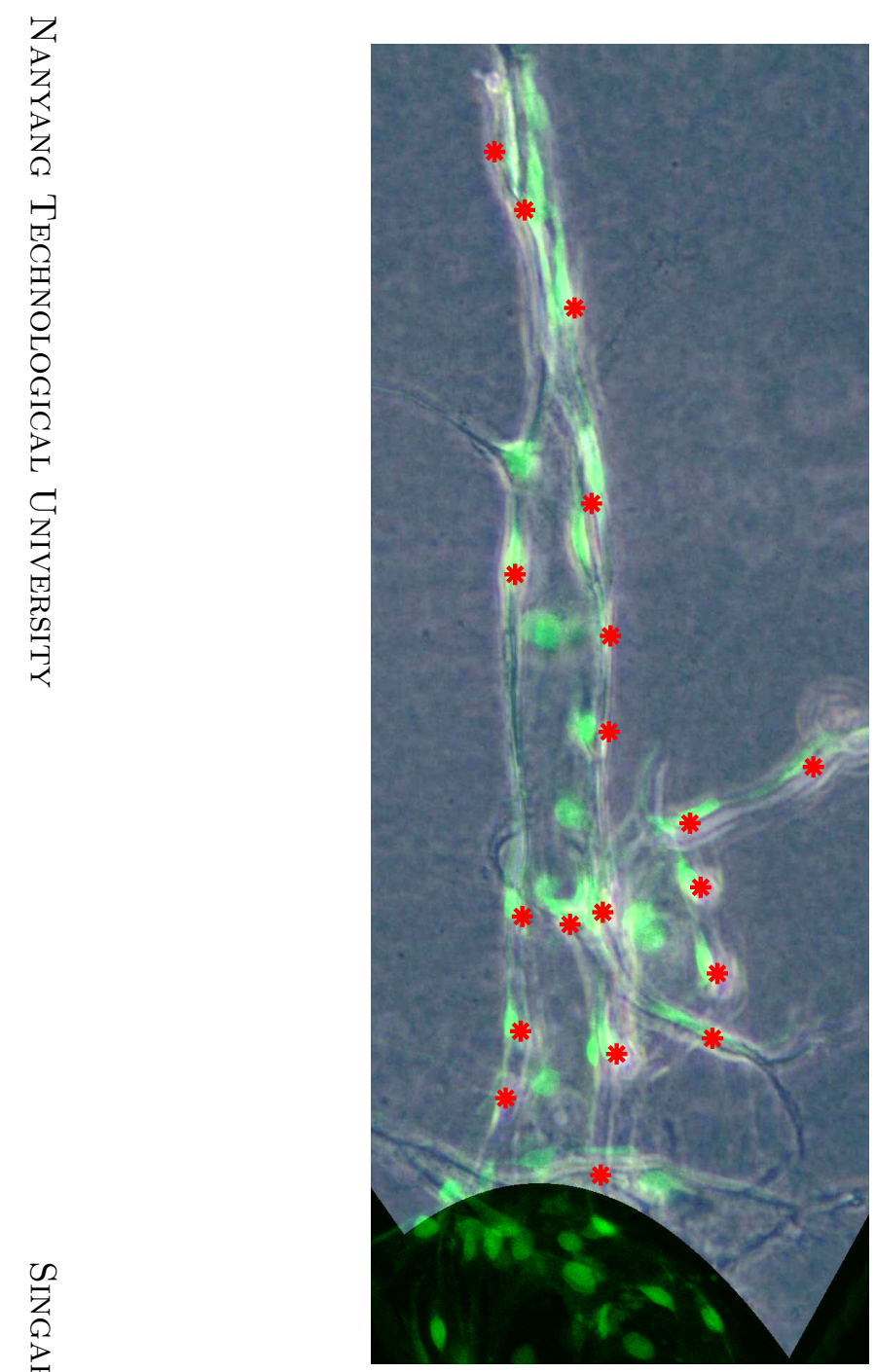

(a) Example I: proposed approach.

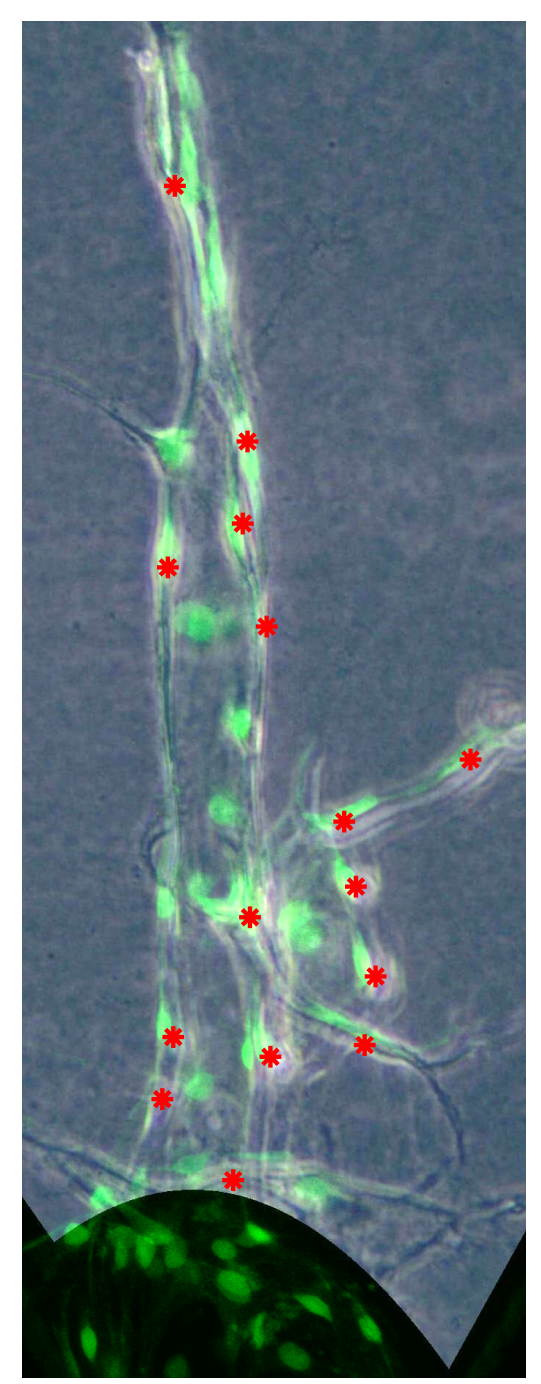

(b) Example I: SVM.

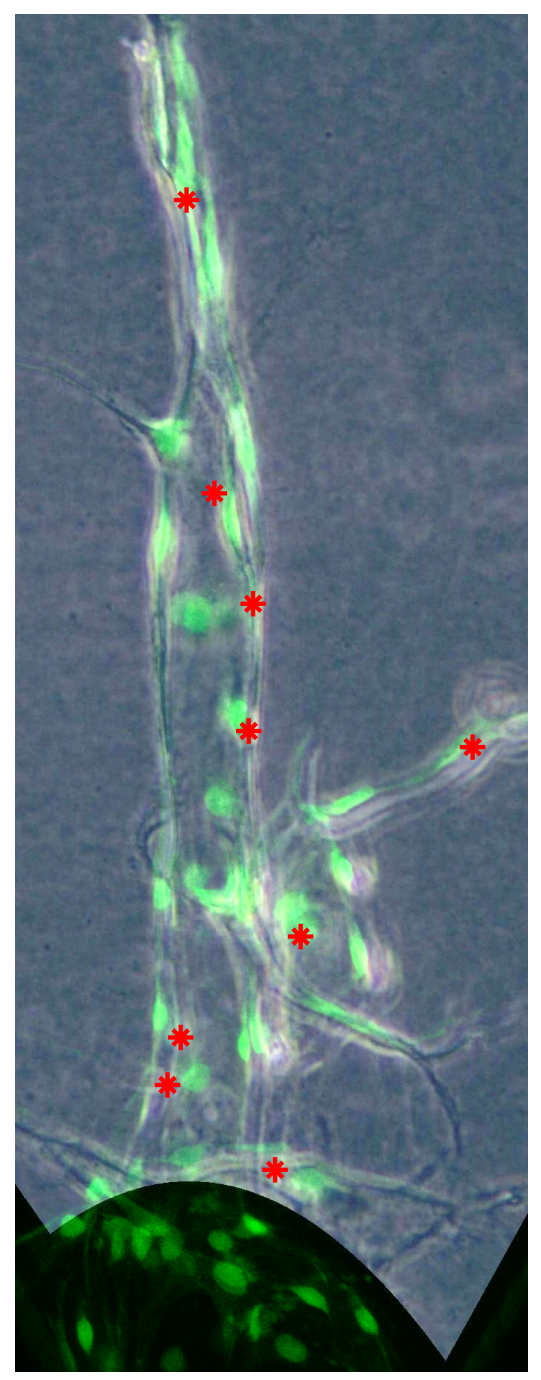

(c) Example I: PCA+SVM. 


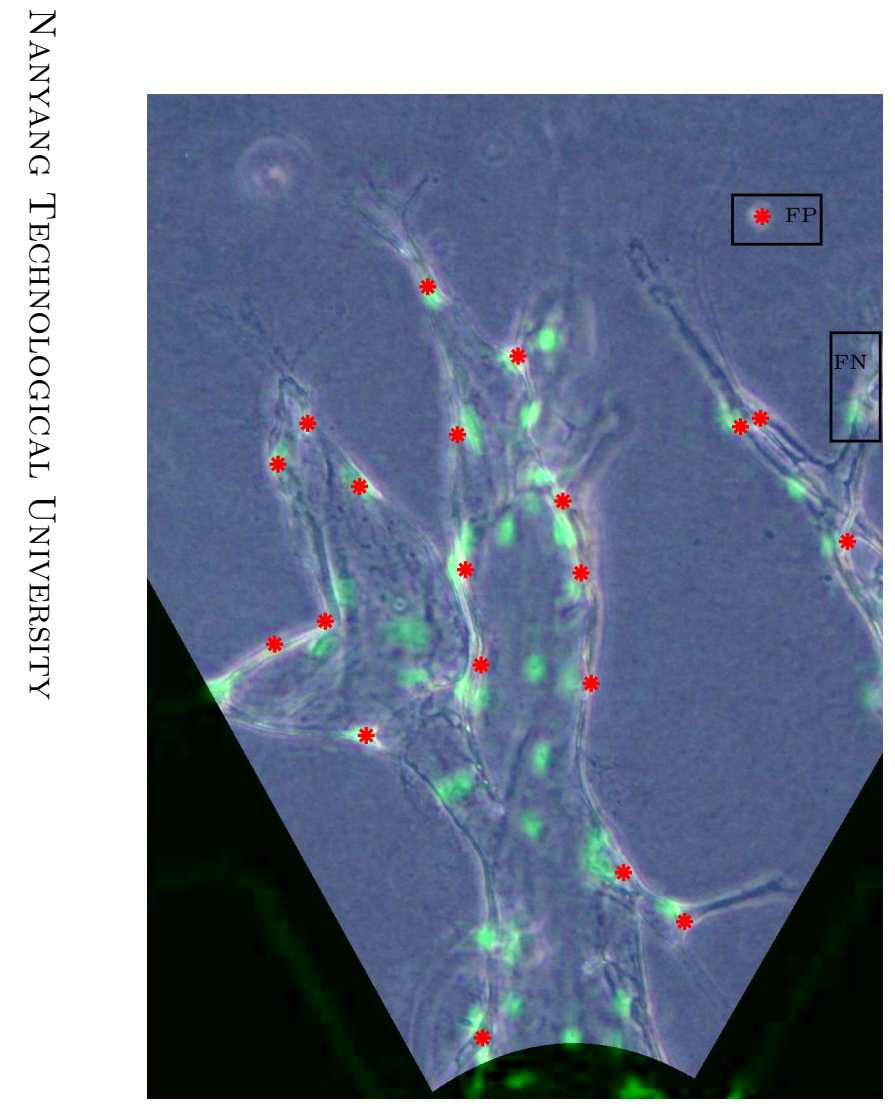

(d) Example II: proposed approach.

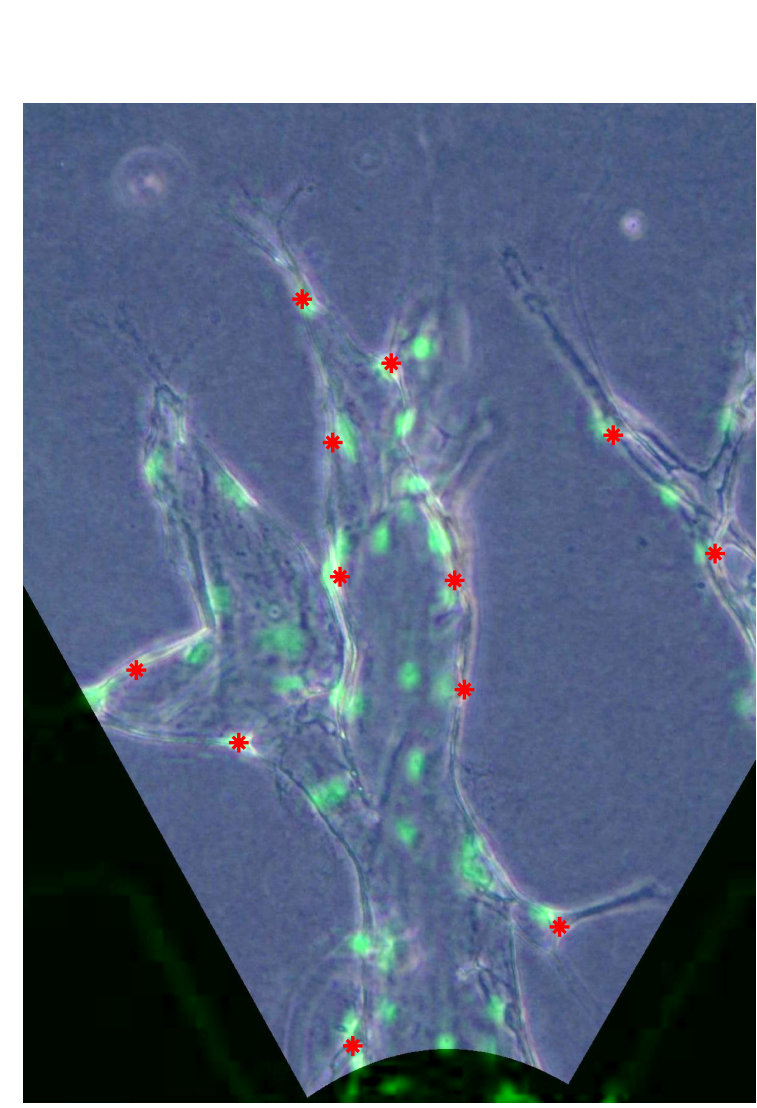

(e) Example II: SVM.

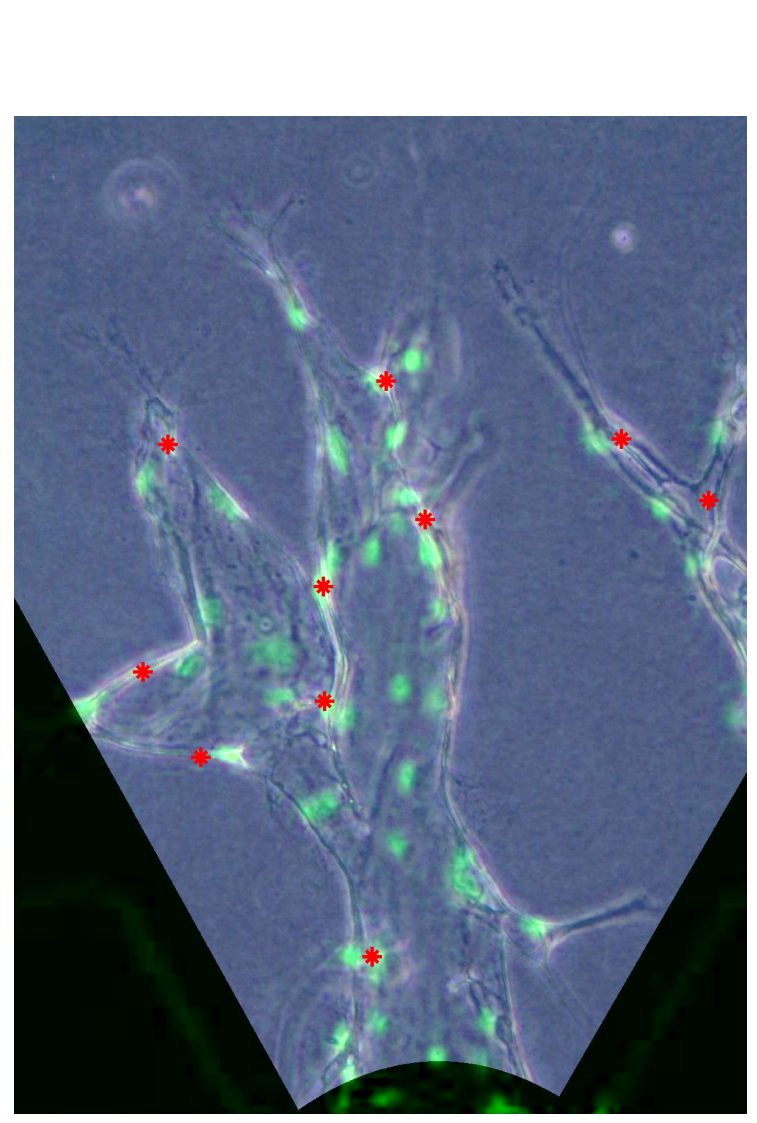

(f) Example II: PCA+SVM.

Figure 3.19: Examples of cell detection results for different approaches: (a) and (d) from the proposed cell detection approach using PLSR; (b) and (e) from an SVM classifier based on original high-dimensional template data; (c) and (f) from an SVM classifier based on the dimension-reduced features after PCA. Red stars $(*)$ indicate the detected nuclei locations. Examples of FN and FP are labeled in (d). 
confocal image.

The TP, FP, and FN values for the three approaches are provided in Table 3.2. Their corresponding precision and recall values are listed in Table 3.3.

Table 3.2: TP, FP, and FN values for different cell detection approaches.

\begin{tabular}{|c|c|c|c|c|c|c|}
\hline & \multicolumn{3}{|c|}{ Case 1: All slices } & \multicolumn{3}{c|}{ Case 2: One slice } \\
\hline Detection Approaches & TP & FP & FN & TP & FP & FN \\
\hline Proposed approach: PLSR & 215 & 29 & 51 & 215 & 29 & 23 \\
\hline SVM & 124 & 23 & 142 & 124 & 23 & 114 \\
\hline PCA \& SVM & 107 & 26 & 159 & 107 & 26 & 131 \\
\hline
\end{tabular}

Table 3.3: Classification performance of cell detection (in \%).

\begin{tabular}{|c|c|c|c|c|}
\hline & \multicolumn{2}{|c|}{ Case 1: All slices } & \multicolumn{2}{c|}{ Case 2: One slice } \\
\hline Detection Approaches & Precision & Recall & Precision & Recall \\
\hline Proposed approach: PLSR & 88.1 & 80.8 & 88.1 & 90.3 \\
\hline SVM & 84.4 & 46.6 & 84.4 & 52.1 \\
\hline PCA \& SVM & 80.5 & 40.2 & 80.5 & 45.0 \\
\hline
\end{tabular}

For case 1, the precision values for all three approaches are above $80 \%$, suggesting that most of the detected cells are actual cells. The recall for PLSR is much higher than for the other two approaches, indicating that our proposed approach (PLSR) detects most of the actual cells from the experimental phase contrast images while the other two approaches fail to do so.

The recall of the proposed approach (PLSR) is limited to about $81 \%$, which can be explained as follows. The height of our device is around $120 \mu \mathrm{m}$ and there are cells migrating throughout this range. We only acquire the 2D image of the angiogenic vessels in focus. Some cells, which are external to the vessels, are out of focus and almost invisible in the phase contrast images. These cells are not detected by our approach. However, these out-of-focus cells are observed in the 3D confocal images. This scenario can be seen from Fig. 3.18.

For case 2, the recall for all the three approaches increases as shown in Table 3.3. Particularly, the recall for the proposed approach (PLSR) increases to 90\%. 
Therefore, we conclude that PLSR is a preferred approach to detect the ECs within 3D angiogenic vessels from the experimental phase contrast images. We hypothesize that PLSR yields more salient features to predict the output, since the dimensionality reduction is carried out with the aim of predicting the output data. By contrast, in PCA dimensionality reduction is performed without knowledge of the output data.

\subsubsection{Tracking Results}

Since our cell association approach is detection-based, we use the cell detection results from the proposed approach (PLSR) as the cell locations during cell association.

We provide two examples to illustrate the association results in Fig. 3.20 and Fig. 3.21 respectively. The results for our proposed tracking system, which combines MHT with backward Kalman filter, are depicted in Fig. 3.20(a) and 3.20(b), and Fig. 3.21(a) and 3.21(b). We also apply backward Kalman filter with validation gating for cell association. The results are shown in Fig. 3.20(c) and 3.20(d), and Fig. 3.21(c) and 3.21(d). Each image presents the tracking results for two consecutive frames and the background is the experimental phase contrast image. We can see that the proposed multi-cell tracking approach produces better tracking results than backward Kalman filter with validation gating.

We applied the proposed system to track the ECs in 5 sprouting regions, where each sprouting region has 4 to 5 frames (one frame per day). We estimated and compared the association accuracies for the proposed approach, the "nearest-neighborhood" association, the backward Kalman filter with validation gating, and the Hungarian method [97] in Table 3.4. We obtained the ground truth through manual association, which is the traditional method of analyzing the cell migratory behaviors.

Since the sampling interval is one day, cells migrate over a relatively long distance 


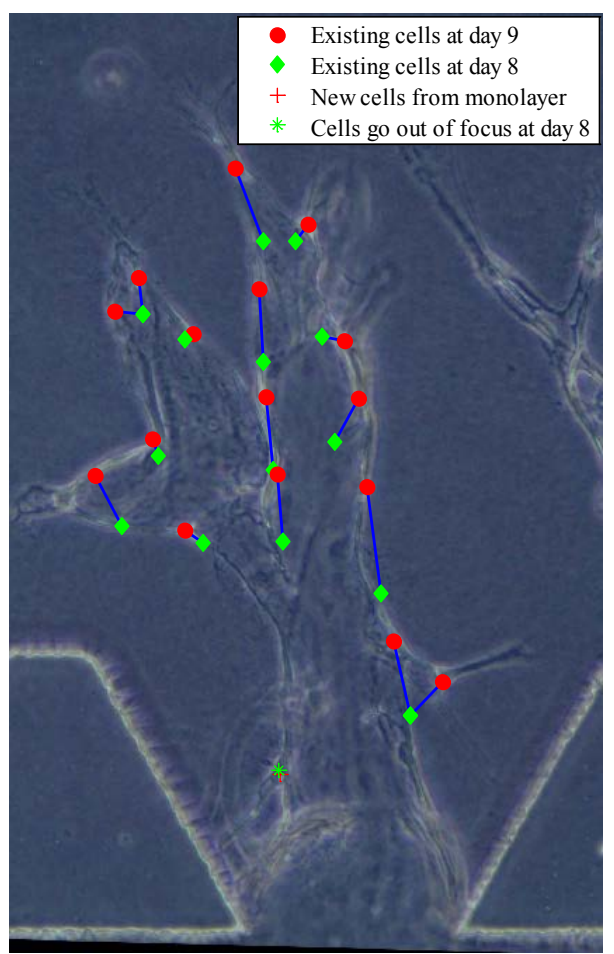

(a) Day 9 to 8.

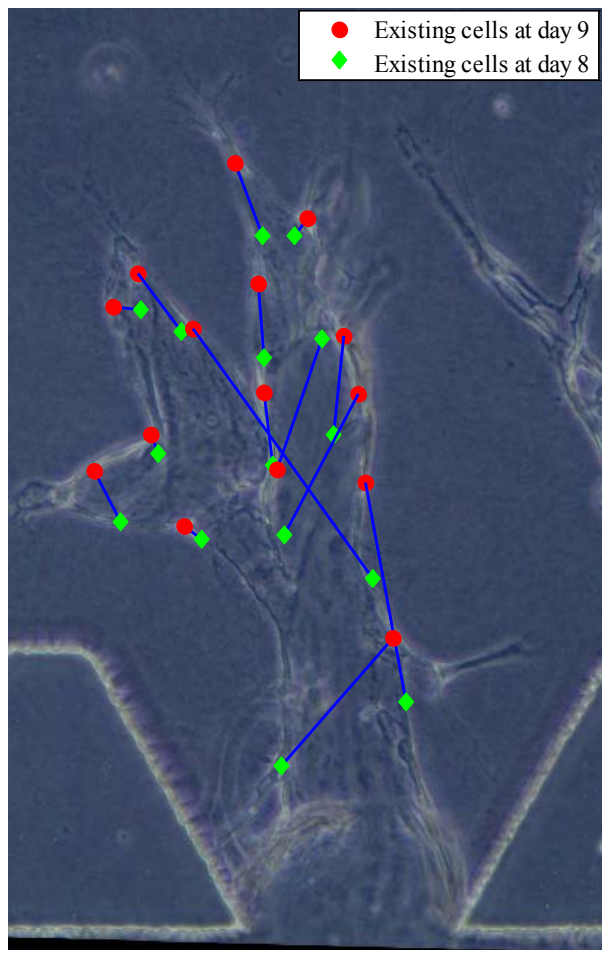

(c) Day 9 to day 8 .

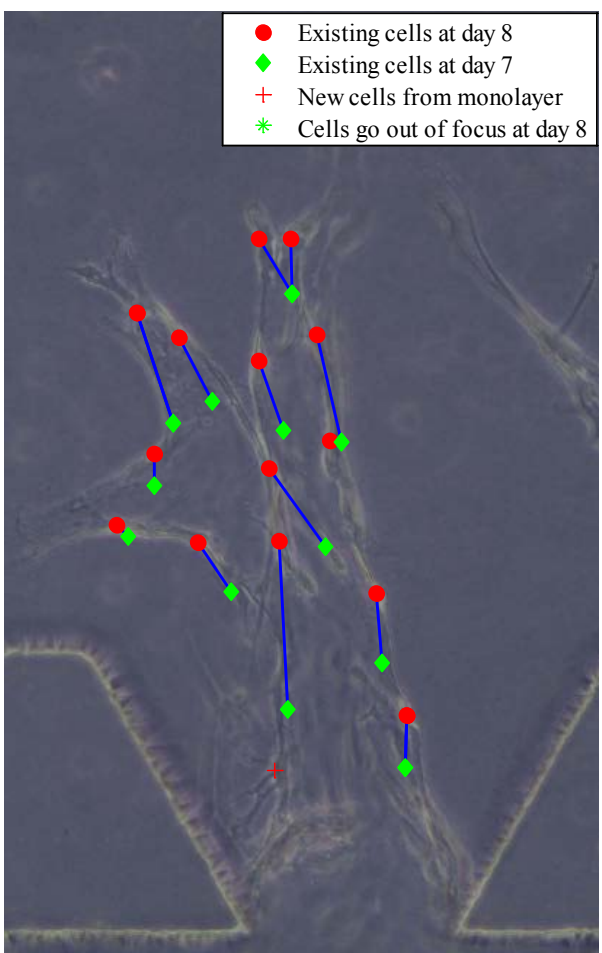

(b) Day 8 to day 7 .

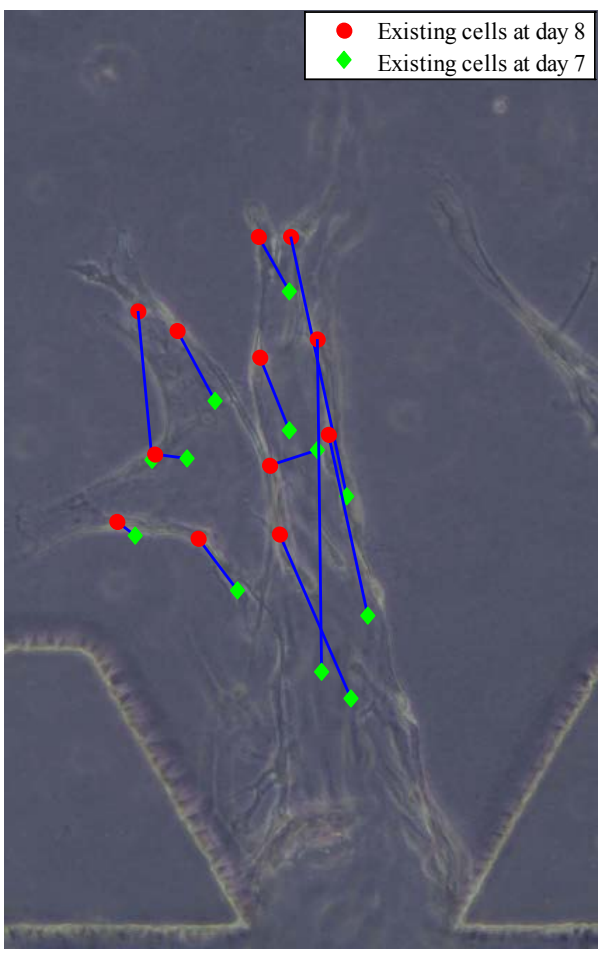

(d) Day 8 to day 7 .

Figure 3.20: Cell tracking example I. (a) and (b): Backward Kalman filter with MHT; (c) and (d): backward Kalman filter with validation gating. Each image shows the outcomes of the tracking algorithms for two consecutive frames, where the phase contrast image is shown in the background.

NANYANG TECHNOLOGICAL UNIVERSiTy 


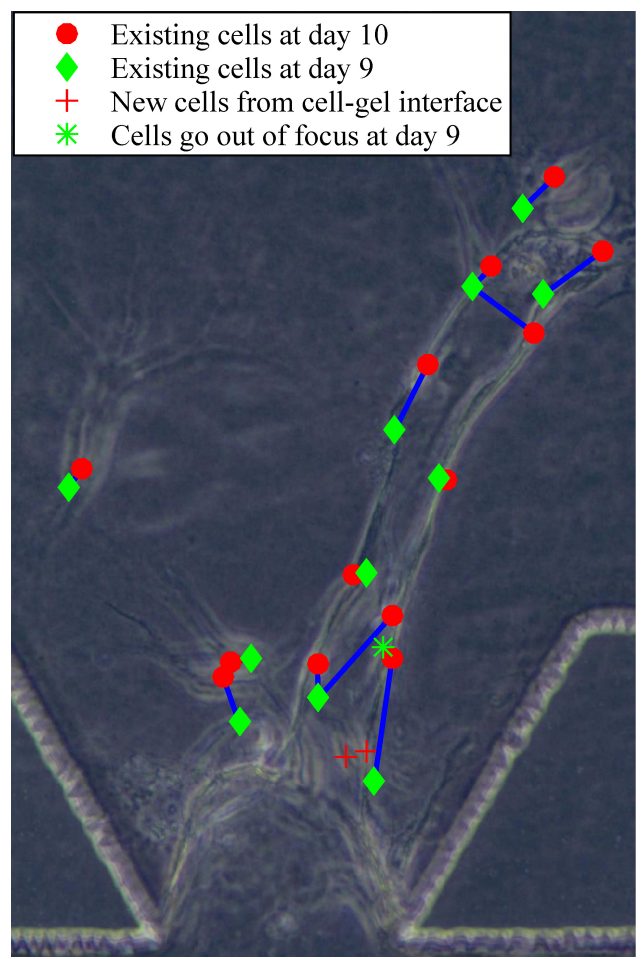

(a) Day 10 to day 9 .

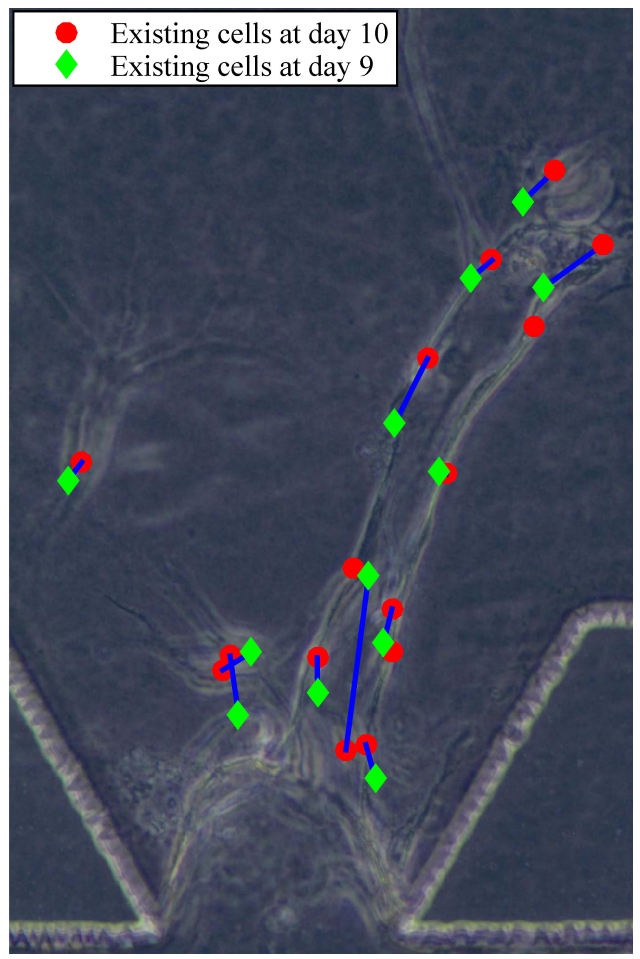

(c) Day 10 to day 9 .

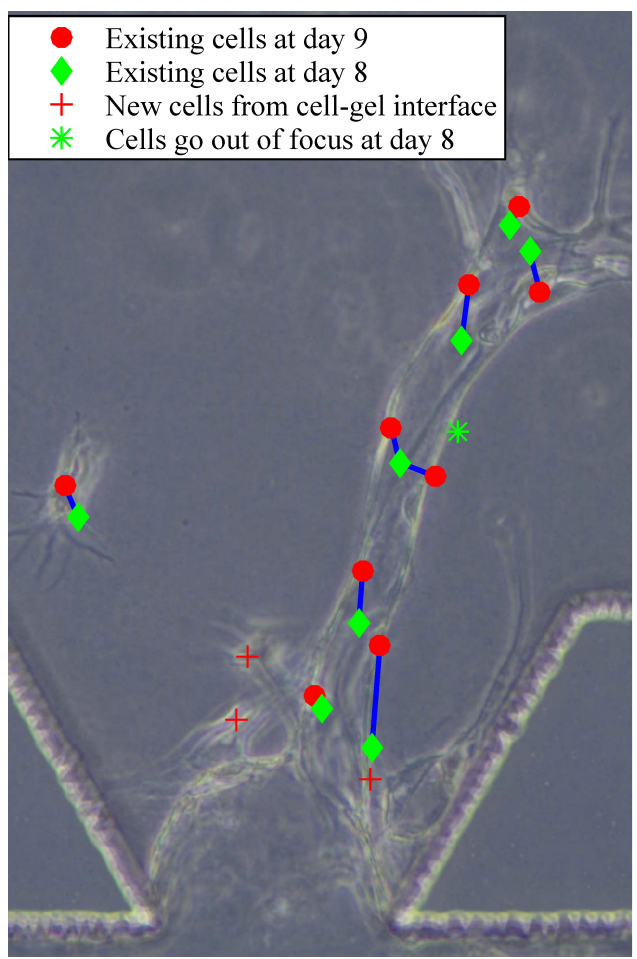

(b) Day 9 to day 8 .

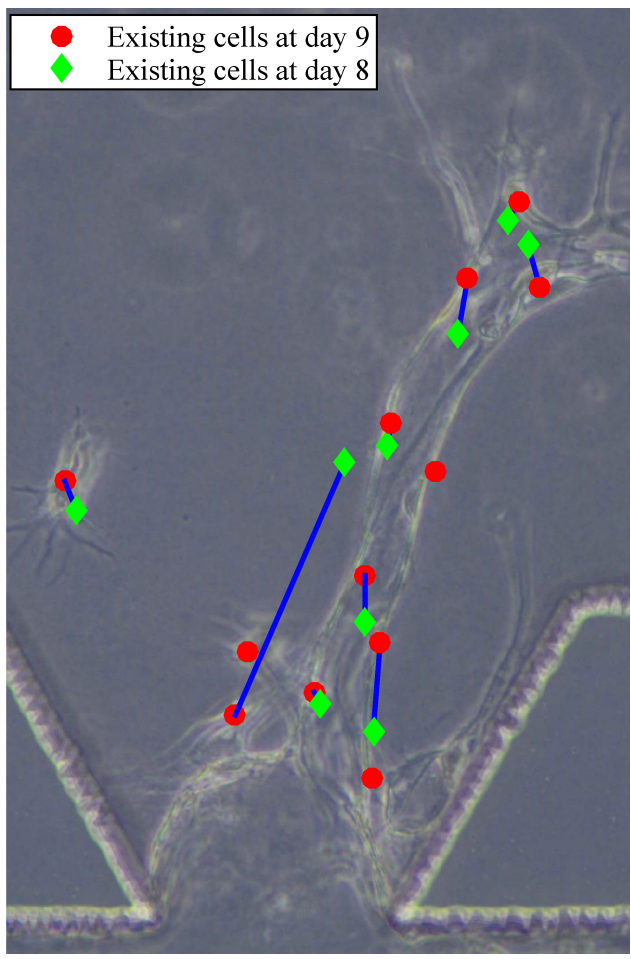

(d) Day 9 to day 8 .

Figure 3.21: Cell tracking example II. (a) and (b): Backward Kalman filter with MHT; (c) and (d): backward Kalman filter with validation gating. Each image shows the outcomes of the tracking algorithms for two consecutive frames, where the phase contrast image is shown in the background.. 
Table 3.4: Cell association accuracy for different approaches: I. Nearestneighborhood association; II. Kalman filter with validation gating; III. the Hungarian method; and IV. Multiple hypothesis Kalman filtering.

\begin{tabular}{|c|c|c|c|}
\hline & Correct association & Total associations & Association accuracy \\
\hline I & 48 & 178 & $27.0 \%$ \\
\hline II & 107 & 178 & $60.1 \%$ \\
\hline III & 94 & 178 & $52.8 \%$ \\
\hline IV & 153 & 178 & $86.0 \%$ \\
\hline
\end{tabular}

between frames. Using "nearest-neighborhood" association, the new tracks at time $k$ are typically associated wrongly to cells that are near to the cell-gel interface at time $k-1$. However, by predicting the tracks backwards each time step via a constant velocity motion model, we are more likely to associate the tracks to the correct observations with a backward Kalman filter with validation gating. Thus, by using the backward Kalman filter, the association accuracy improves from $27.0 \%$ from $60.1 \%$. In the Hungarian method, we use the Euclidean distances of cells between frames to find the associations with minimal cost. However, cell migration and proliferation are not considered since it only allows for one-to-one matching. Thus only $52.8 \%$ association accuracy is obtained.

In our approach, we combine MHT with backward Kalman filter, which delays the decision making and determines the associations when sufficient information is available. Moreover, biological knowledge is incorporated when considering the new cells from the monolayer and reducing the number of new tracks. As a result, we can track not only the migration and proliferation of the existing cells but also the new cells from the monolayer and out-of-focus plane. This explains why the association accuracy increased to $86.0 \%$ for our approach.

Cell trajectory information can be obtained from the tracking results. Moreover, the system can automatically generate cell lineage plots (see Fig. 3.22), showing the histories of the cell proliferations and cell migrations into the gel, with timestamps of when the cells are in-focus and out-of-focus. Such cell lineage plot provides 
crucial information (e.g., the number of cells, division time, and growth fraction) to biologists who are interested in studying cell migration behaviors in angiogenesis under different conditions.

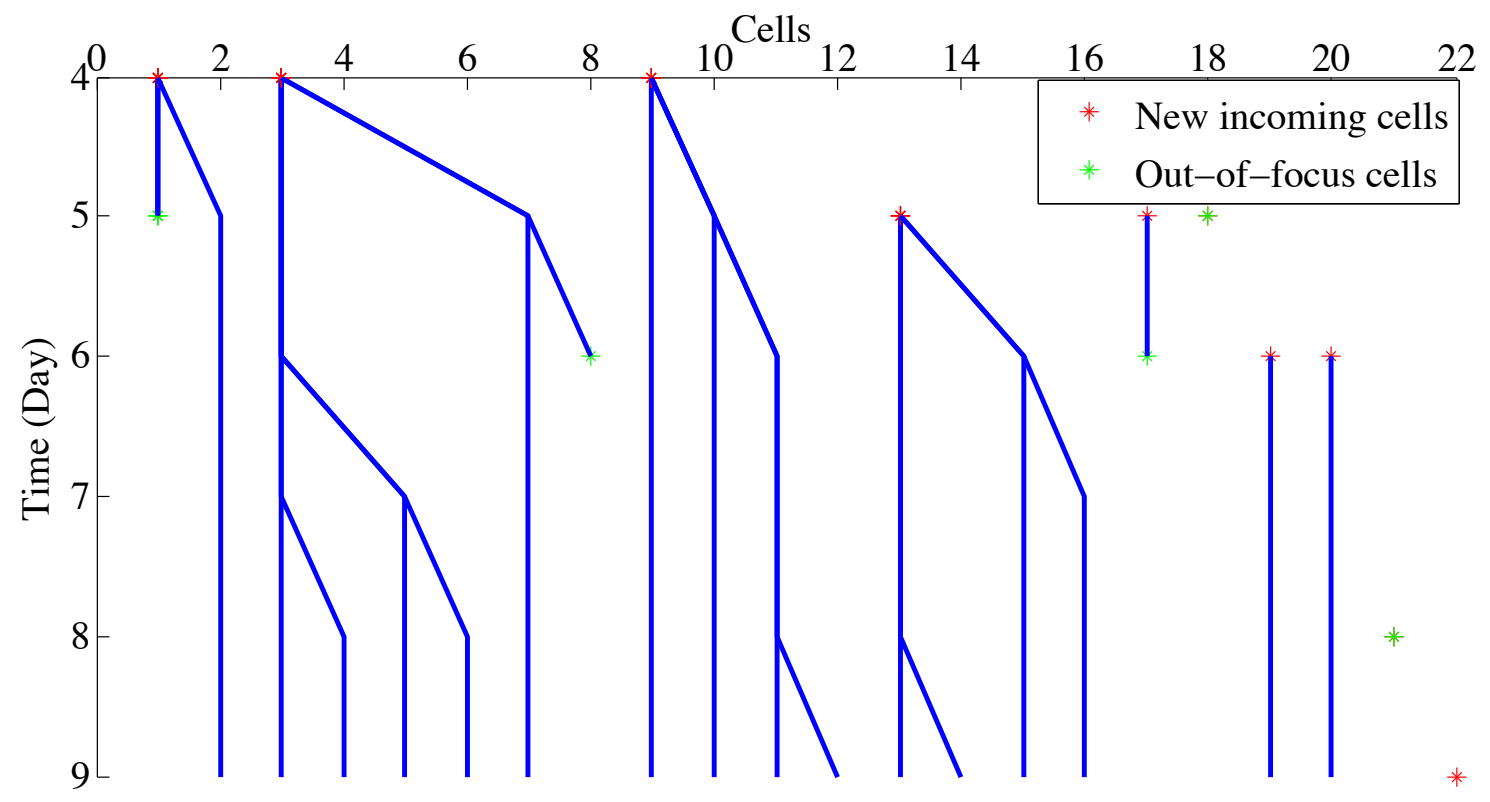

(a) Example 1.

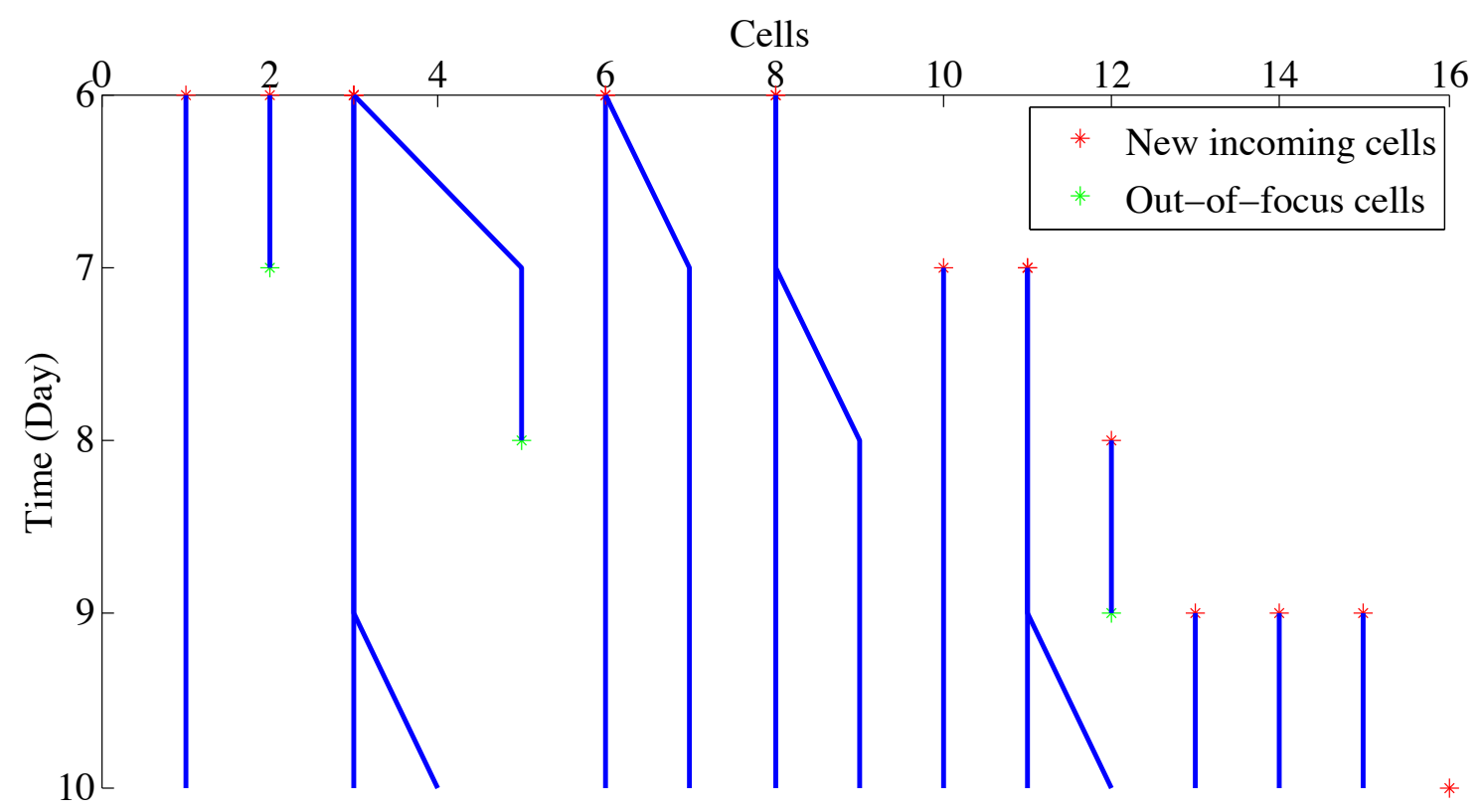

(b) Example 2.

Figure 3.22: Cell lineage plots. The blue lines represent the cells that migrate within the 3D gel; the red stars represent the incoming cells from the cell-gel interface, whereas the green stars indicate the cells migrating out-of-focus. 
The cell lineage plots in Fig. 3.22(a) and Fig. 3.22(b) correspond to the two examples in Fig. 3.20 and Fig. 3.21 respectively. The blue lines indicate that the cells are in-focus and migrate within the 3D gel. The branch points of these blue lines represent cell proliferations. The red stars illustrate the incoming cells from the cell-gel interface. The green stars indicate that cells migrate out-of-focus. The cell lineage plots illustrate the histories of cell migration and proliferation in a compact and effective manner.

Under different growth conditions, cells may express different biomarkers, which can be identified through fluorescent staining in the end-point images. From the cell lineage and trajectory information, biologists can discover links between the chemical and physical characteristics of a cell.

\subsection{Conclusion}

We presented the AMCTS to track the ECs migration within the angiogenic vessels formed in 3D MFDs by combining end-point confocal images and time-lapse phase contrast images. This system consists of preprocessing, cell detection, and multiple hypothesis Kalman filtering. We incorporate biological knowledge, such as time of cell cycle and experimental device properties, to facilitate cell association. Our proposed cell detection approach (PLSR) yields a precision of $88.1 \%$ and a recall of $90.3 \%$ for EC candidates detection, which helps the biologists to recognize large number of cells automatically with high accuracy. The association accuracy reaches $86.0 \%$ for the proposed multiple hypothesis Kalman filtering approach, suggesting that our proposed system associates cells more accurately than the commonly used methods for cell association and tracking.

From the tracking results in Fig. 3.20 and Fig. 3.21, we are able to obtain information about the cell trajectories and create cell lineage plots (see Fig. 3.22) to visualize the histories of the cell migration and proliferation in a compact and effec- 
tive manner. With this information about the cell histories, mathematical models can be developed to predict the cell migration under different conditions, leading ultimately to a better understanding of the biological processes in angiogenic cell migration. This system can be also used for a cell harvesting robot to automatically select which cell to harvest. 


\section{Chapter 4}

\section{Automatic Tracking of Angiogenic Vessel Formation}

Summary - In this chapter, we present an automated vessel formation tracking system (AVFTS) to track angiogenic vessel formation over time and to extract the geometric parameters such as the average vessel length and width, and the number of branches from the time-lapse experimental phase contrast images. The proposed system consists of preprocessing, skeletonization, and branch tracking. Firstly, we preprocess the experimental images to obtain the binary vessel shapes through stitching, image registration, and vessel segmentation. Next, we apply a distance transform (DT) and an augmented fast marching method (AFMM) to extract the connected skeleton segments. A support vector machine (SVM) classifier is then trained to remove the specious skeleton segments that are not part of vessels. Lastly, after determining the base nodes and identifying the routes for the main branches, we associate the branches over time to obtain the branch trajectories for the angiogenic vessels. Specifically, we elaborate on the importance of branch formation tracking in Section 4.1. In Section 4.2, we explain the proposed system in detail. The proposed system is also applicable to distinguish tip cells and stalk cells based on the relative cell positions within a branch. Moreover, we extract and compare the morphological 
difference for the angiogenic vessels under different S1P conditions. We present the numerical results for vessel formation tracking and the applications of the proposed system in Section 4.3. We offer concluding remarks in Section 4.4.

\subsection{Introduction and literature review}

Sprouting angiogenesis is a highly dynamic process involving both cell-cell interactions and cell-ECM interactions [4, 112, 113]. Tip cells and stalk cells can dynamically switch their phenotypes and functions to form new branches and reconnections during the sprouting process $[19,20]$. The inter-transition of the cell phenotypes plays an important role for ECs sprouting out from monolayer, extending and creating new branches, as well as reconnecting in a later stage [21, 22, 23].

As discussed in Section 1.2, the ECs in the MFDs migrate within the 3D collagen gel, remodel the gel, and form 3D angiogenic vessels after long-term culture. Due to the existence of tight cell junctions, these ECs do not overtake each other and their migrations are constrained by the angiogenic vessels and the collagen gel. The topological properties of the angiogenic vessels are regulated by cell migration, cellcell interactions, and cell-ECM interactions [114, 115]. Quantitative analysis of the angiogenic vessel formation would benefit for the understanding of the collective cell behaviors in angiogenesis [116]. Moreover, computational models can be generated based on the parameter values from the experimental data for the mathematical simulation of angiogenesis [117].

However, most of the existing automated image processing systems are developed for studying individual cell migration [66, 81, 63, 64, 94, 96, 105]. Only a few methods exist to quantitatively analyze the formation of the angiogenic networks, providing geometric parameters such as the average length, the number of branches, and the number of nodes $[118,119,120,121,122]$. However, the networks in these studies are formed by culturing HUVECs in 2D in vitro systems, which do not form lumens. 
Consequently, quantitative analysis tools for angiogenic vessels formed in 3D in vitro cell culture systems still need to be developed.

Quantitative angiogenic vessel parameters are commonly estimated from the vessel skeleton, since it provides a simple and compact representation of the angiogenic vessel. Common methods for the extraction of the skeleton, namely skeletonization, are divided into two classes: morphological thinning and distance coding [123]. Morphological thinning removes the outer layers of pixels from the vascular networks successively while maintaining connectivity [124, 125]. This methodology has been implemented in [120], [121], and [122] to quantify the EC networks in 2D in vitro cell culture system. However, it is time-consuming and very sensitive to defects in the boundaries. The distance coding approach extracts skeletal points directly from the image based on the DT [126, 127, 128, 129, 130]. By implementing Fast Marching Method (FMM) to evolve the boundaries in normal direction, the skeleton lying along the singularities in the DT can be obtained [131, 132]. However, the computation of the singularities of the DT is a numerically unstable and delicate process.

In addition, the dynamic selection and continued competition of cell phenotypes (tip and stalk cells) is still not fully understood [15]. Identification of cell phenotypes from experimental images would be useful for unraveling the biological functions of different cell phenotypes and developing computational models.

Therefore, the objective of this chapter is to develop an automated image analysis system to track angiogenic vessel formation, to extract the geometric parameters such as the average vessel length, width, and the number of branches, as well as to identify cell phenotypes (tip/stalk cells) from the time-lapse experimental phase contrast images. 


\subsection{Automated Vessel Formation Tracking Sys- tem}

Fig. 4.1 illustrates the proposed AVFTS, which consists of preprocessing, skeletonization, and branch tracking. The first step is to obtain binary vessel shapes by preprocessing the experimental phase contrast images through stitching, image registration, and vessel segmentation. Skeletonization, providing a simple representation of the vessel shape, is a crucial step in this system. We apply DT and AFMM on the binary vessel shapes to extract the skeletons of the angiogenic vessels. Fig. 4.2(a) shows one example of the extracted skeletons composing of a set of nodes and segments. Specifically, the yellow star is branch node and the 3 green stars are end nodes. The blue lines connecting two nodes are defined as segments. Each branch node connects to at least 3 other neighboring nodes by segments, and each end node connects to only 1 neighboring node. Fig. 4.2(b) illustrates an individual segment with branch node (yellow star), end node (green star), segment (blue line), and the vessel radii (red circles). Next, we label each segment by a trained SVM classifier in order to remove the specious segments which are not part of vessels. Lastly, we determine the base nodes, identify the routes for the main branches by joining the connected segments, and apply the Hungarian method to associate the branches over time in order to obtain the branch trajectories for the angiogenic vessels. The geometric parameters for the angiogenic vessels including the average vessel length/width, the number of branches, and the number of nodes are also extracted.

\begin{tabular}{|c|c|c|c|c|}
\hline Acquired & $\begin{array}{c}\text { Preprocessing } \\
\text { Stitching }\end{array}$ & $\begin{array}{c}\text { Skeletonization } \\
\text { DT }\end{array}$ & $\begin{array}{c}\text { Branch Tracking } \\
\text { Base node determination }\end{array}$ & $\begin{array}{l}\text { Branch tracking } \\
\text { results }\end{array}$ \\
\hline images & $\begin{array}{l}\text { Image registration } \\
\text { Vessel segmentation }\end{array}$ & $\begin{array}{l}\text { AFMM } \\
\text { SVM }\end{array}$ & Hungarian method & $\begin{array}{l}\text { Numerical branch } \\
\text { information }\end{array}$ \\
\hline
\end{tabular}

Figure 4.1: Diagram of the automated vessel formation system: preprocessing to generate binary vessel shape, skeletonization to obtain the connected skeleton segments with branch nodes and end notes, and branch tracking to identify the routes for the main branches and associate the branches over time. 


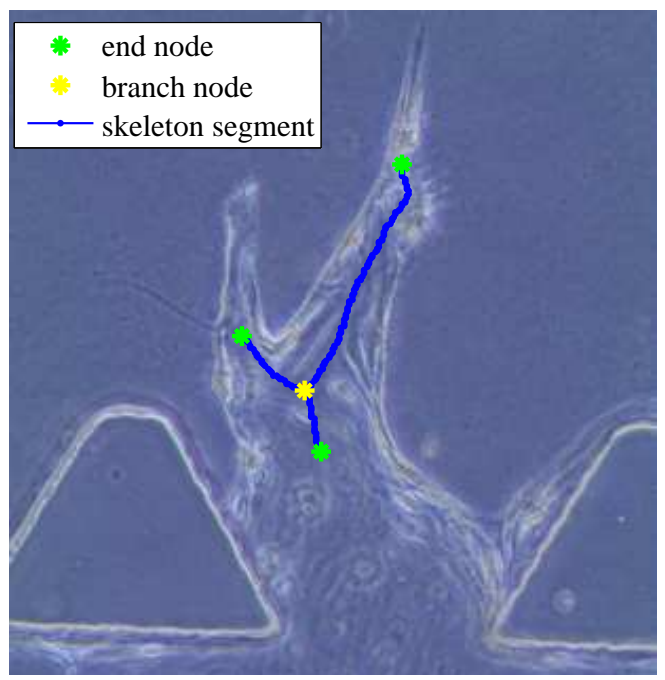

(a) Skeleton segments and nodes.

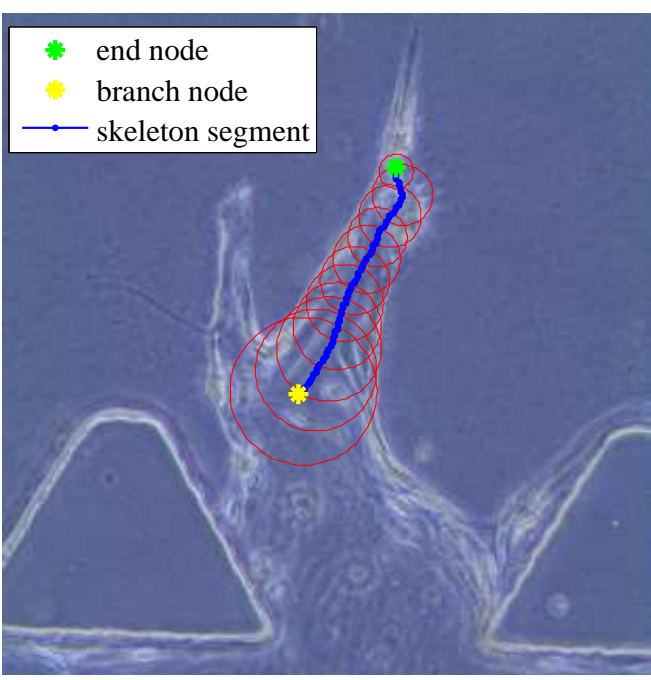

(b) One segment.

Figure 4.2: Example of (a) skeleton segments and nodes and (b) one segment: branch nodes (yellow), end nodes (green), segments (blue lines), and vessel radii (red circles).

The preprocessing step is identical to the one in the AMCTS. It has been discussed thoroughly in Section 3.2.1. In this section, we explain the skeletonization and branch tracking in detail.

\subsubsection{Skeletonization}

After obtaining the binary vessel shapes through preprocessing, skeletonization is applied to quantitatively represent the vessel shapes.

Firstly, DT and AFMM are implemented to extract the connected skeleton segments with branch nodes and end nodes (see Fig. 4.2(a)). We then train an SVM classifier in order to label and remove the specious segments which are due to noise or part of filopodia. 


\subsubsection{Distance Transform and Augmented Fast Marching Method for Skeletonization}

Skeletonization relies on an accurate distance map that can be generated by the DT [125]. For a given object $\Omega$ with the boundary $\delta \Omega$, DT labels each point $p$ of the object by the distance to its closest point $q$ on the boundary. For all the points $p \in \Omega$, DT can be defined by [133]:

$$
T(p)=\min _{q \in \delta \Omega}(\operatorname{dist}(p, q))
$$

where $\operatorname{dist}(p, q)$ is the distance metric. Specifically, Euclidean distance is employed as the distance metric in this chapter, which is computed by:

$$
\operatorname{dist}(p, q)=\|p-q\|_{2}
$$

DT in equation (4.1) can be computed with FMM by evolving the boundaries in normal direction [130]:

$$
|\nabla T|=1
$$

The skeleton lies along the singularities in the DT. To initialize the FMM, we set $T=0$ for all the points on the boundary $\delta \Omega$ and $T=\inf$ for all the points inside the object $\Omega$. Equation (4.3) can be solved by finite difference discretization on a Cartesian grid [131]. However, direct computation of the singularities in the DT is difficult. To compute the skeleton, the FMM algorithm is augmented by one real value $U$ for each pixel point [130]. For the pixel on the boundary $\delta \Omega$, we choose an arbitrary pixel to set its $U=1$ and then monotonically increase $U$ along the boundary. For each pixel inside the object $\Omega$, the value $U$ indicates the parameterized boundary location that it came from, which is obtained from the DT. Fig. 4.3(a) shows the skeleton of a rectangular and Fig. 4.3(b) illustrates the $U$ values for the lower right corner of the rectangular obtained from AFMM. 
After computing the $U$ values for all the pixel points, the skeleton can be obtained by detecting the sharp discontinuities in $U$. In other words, if the $U$ value for one point differs from the $U$ values of its neighboring points by more than a given threshold, it will be retained as a point in the skeleton. We set the threshold to $t_{0}$ in order to prune the skeleton segments caused by boundary details shorter than $t_{0}$ pixels. For our cases, setting the threshold to 100 pixels generates good results. In Fig. 4.3(b), the pixels on the diagonal line labeled in light blue are extracted as skeleton.

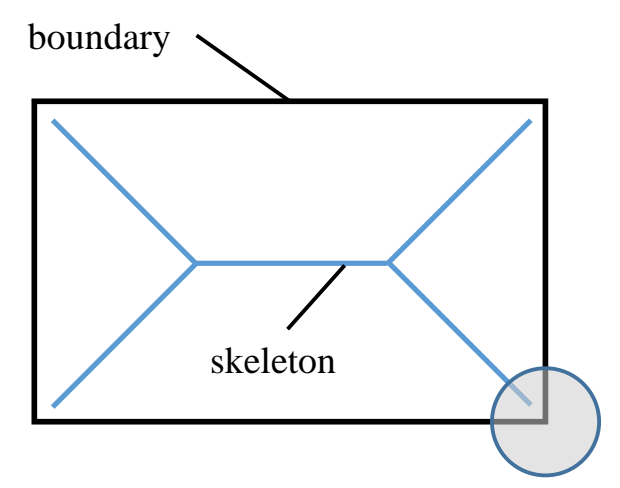

(a) Skeleton of a rectangular.

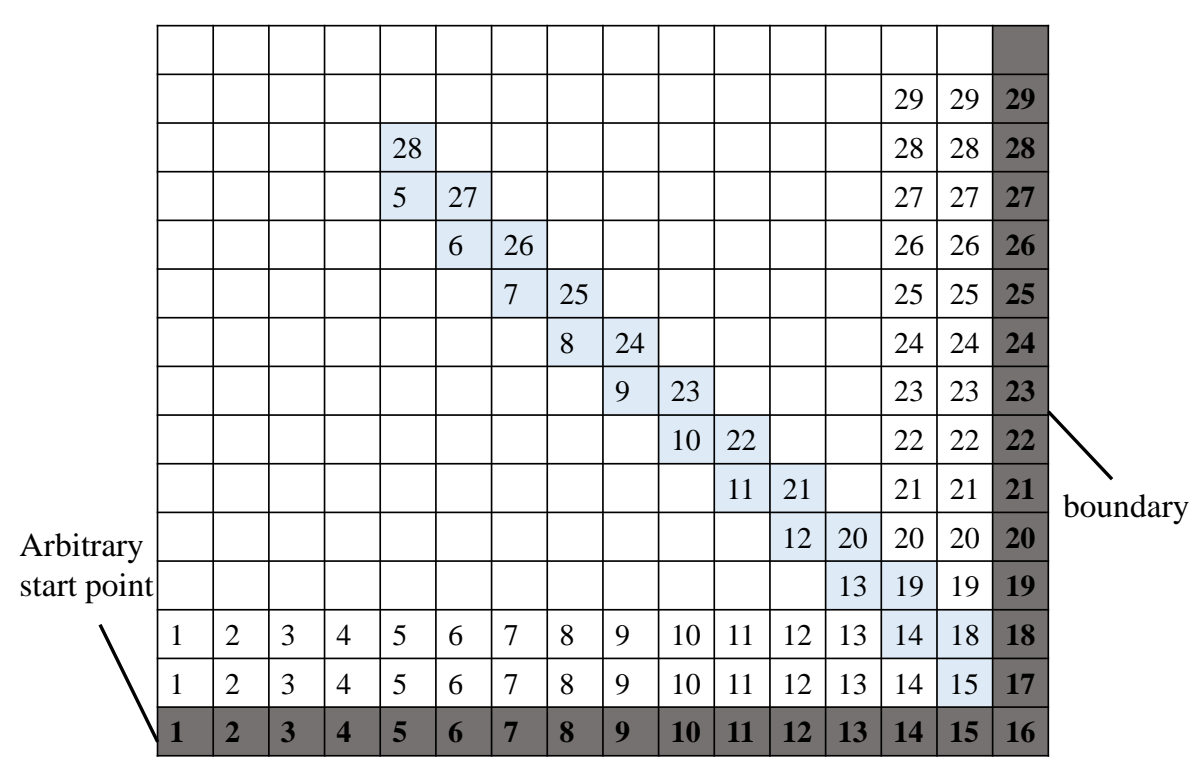

(b) $U$ values of the lower right corner of the rectangular.

Figure 4.3: Demonstration of AFMM algorithm to find the skeleton of a simple rectangular shape.

Fig. 4.4(a) shows an example of the binary vessel shape obtain from the prepro- 
cessing step explained in Section 3.2.1. Computation of the vessel skeleton requires the boundary coordinates of the vessel itself. Thus, we first acquire the vessel boundary by applying the "bwboundaries" function in MATLAB, based on the Moore-Neighbor tracing algorithm [134]. In Fig. 4.4(b), we plot the extracted vessel boundary in green.

Since the vessel boundary is very noisy, applying a standard skeletonization based on iterative thinning algorithm [135] results in a large number of noisy protrusions, as shown in Fig. 4.4(c). We apply the AFMM algorithm to compute the skeleton of the binary vessel shape [130]. By selecting a proper threshold to remove noisy protrusions, we extract the connected skeleton segments, as shown in Fig. 4.4(d). By comparing Fig. 4.4(d) with Fig. 4.4(c), it is clear that the vessel skeleton obtained with the AFMM algorithm is less sensitive to noise in the boundary.

Fig. 4.4(e) shows the connected skeleton segments and nodes in the binary vessel shape, where the 4 branch nodes are plotted as red circles and the 6 end nodes are plotted as green circles. In order to compute the vessel width, we divide each segment into $N_{e q}-1$ equidistant parts and estimate the radii at these $N_{e q}$ points by their minimum distances to the boundaries. A larger $N_{e q}$ leads to more accurate estimation of the vessel width but at the expense of computation time. Therefore we choose $N_{e q}=11$ empirically. In Fig. 4.4(f), we plot the skeleton segments with radii in the original phase contrast image.

\subsubsection{Support Vector Machines for Segment Classification}

Although the skeletonization by DT and AFMM is less sensitive to noise in the boundary compared with morphological thinning, there are still some skeleton segments that are caused by noise or one part of filopodia. By comparing the skeleton segments in the phase contrast images with their 3D confocal images of the stained vessels, we can determine whether a segment represents the angiogenic vessels or not. For example, in Fig. 4.4(g), we can identify that the skeleton segment in the red 


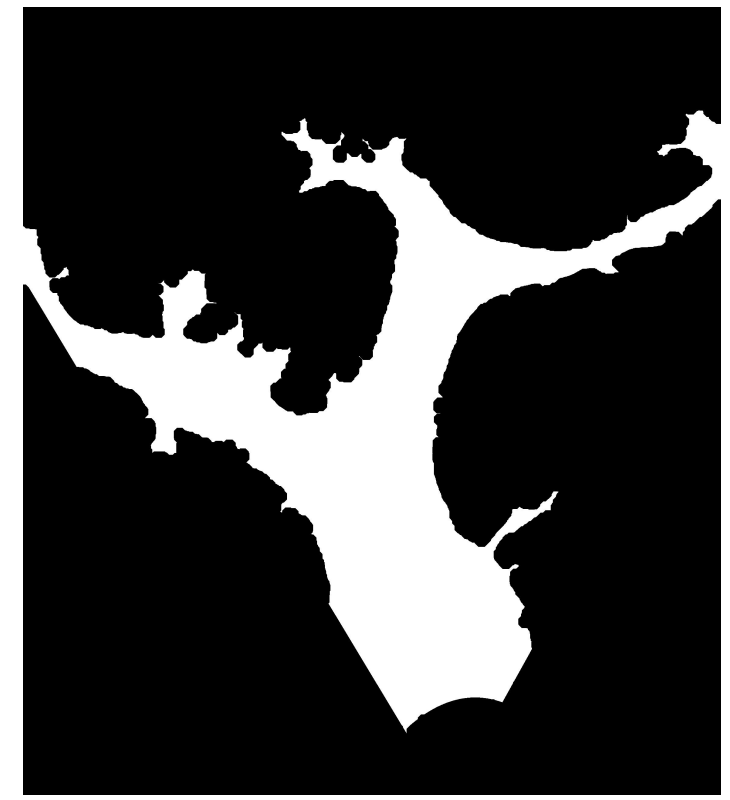

(a) Binary vessel shape.

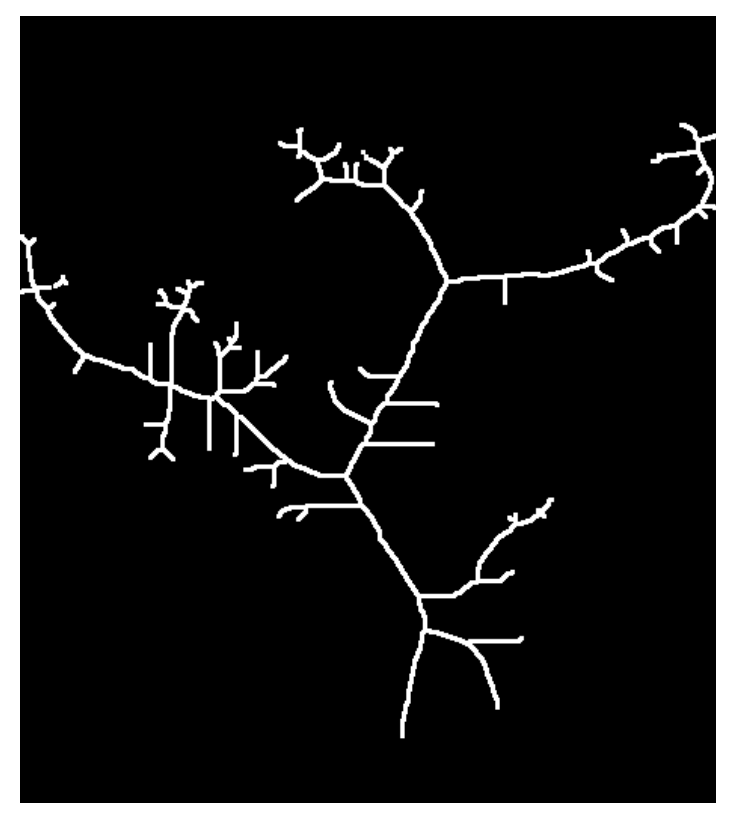

(c) Skeleton from thinning.

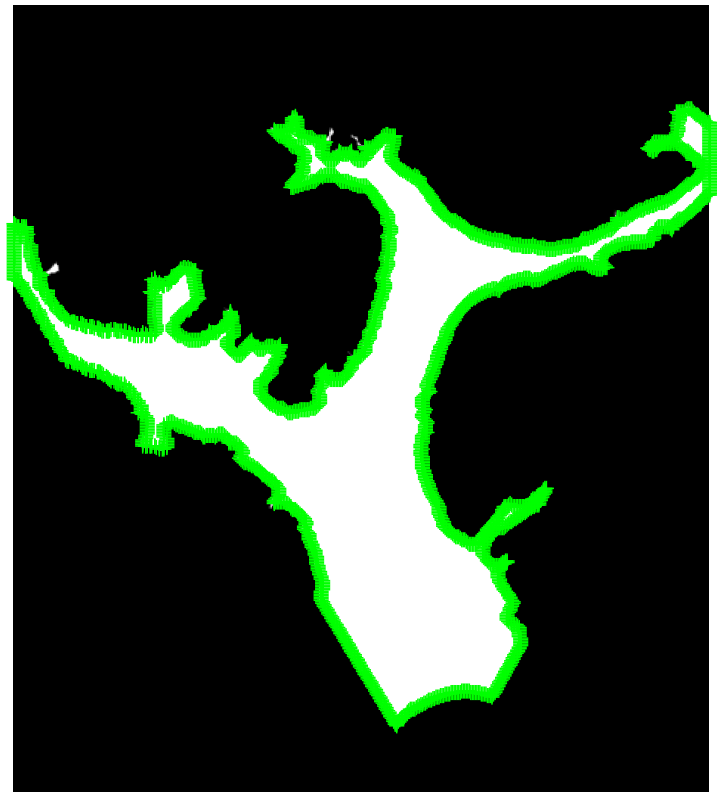

(b) Boundary of the vessel.

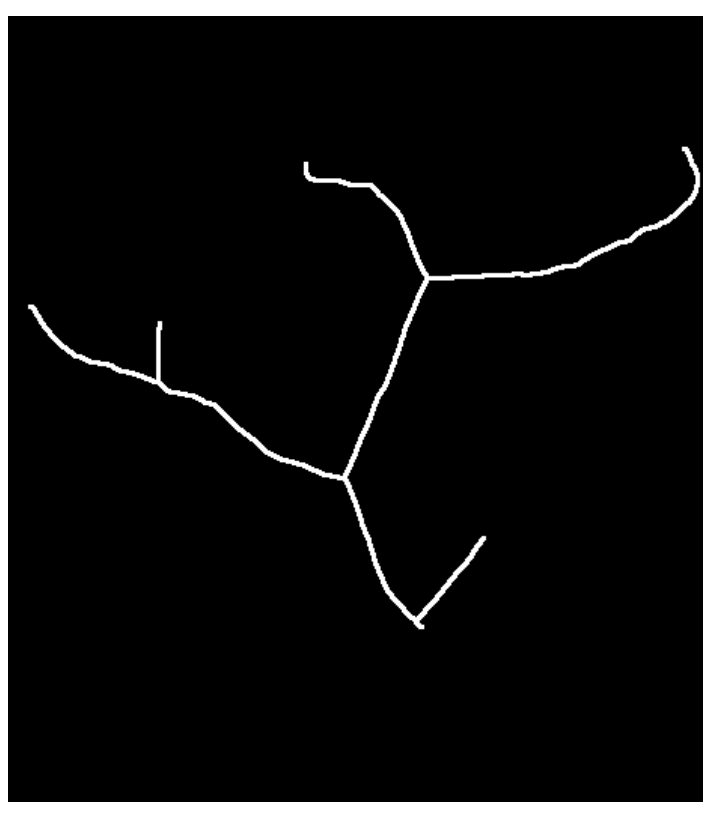

(d) Skeleton from DT and AFMM. 


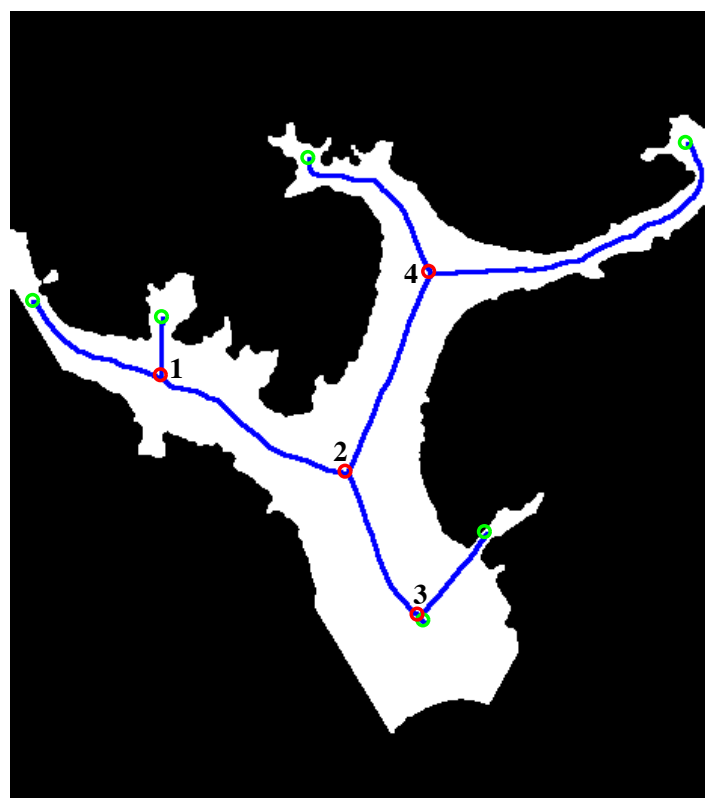

(e) Skeleton segments and nodes: end nodes (green), branch nodes (red), and segments (blue lines) connecting two nodes.

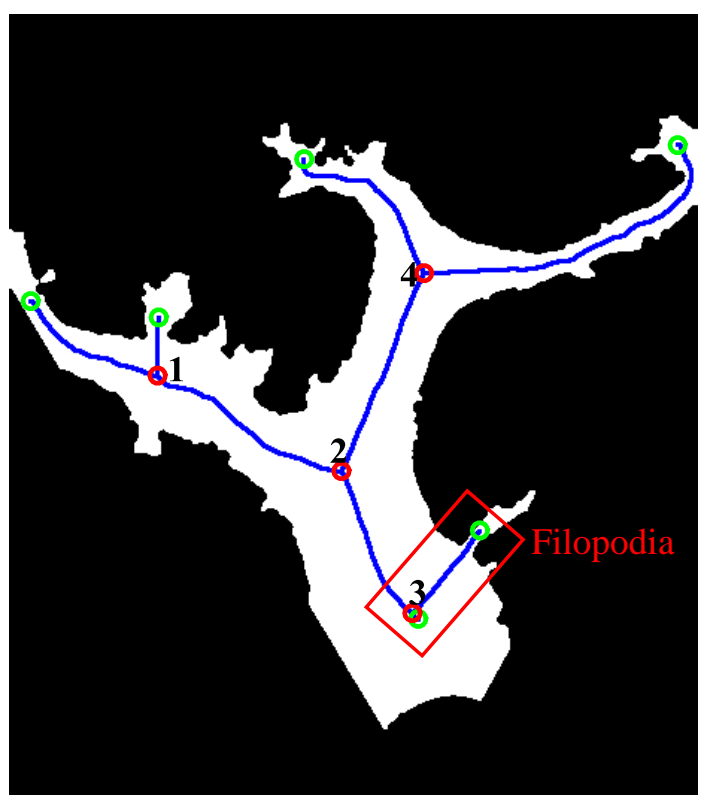

(g) Example of a filopodia wrongly labeled as (h) Skeleton segments with radii after SVM classkeleton.

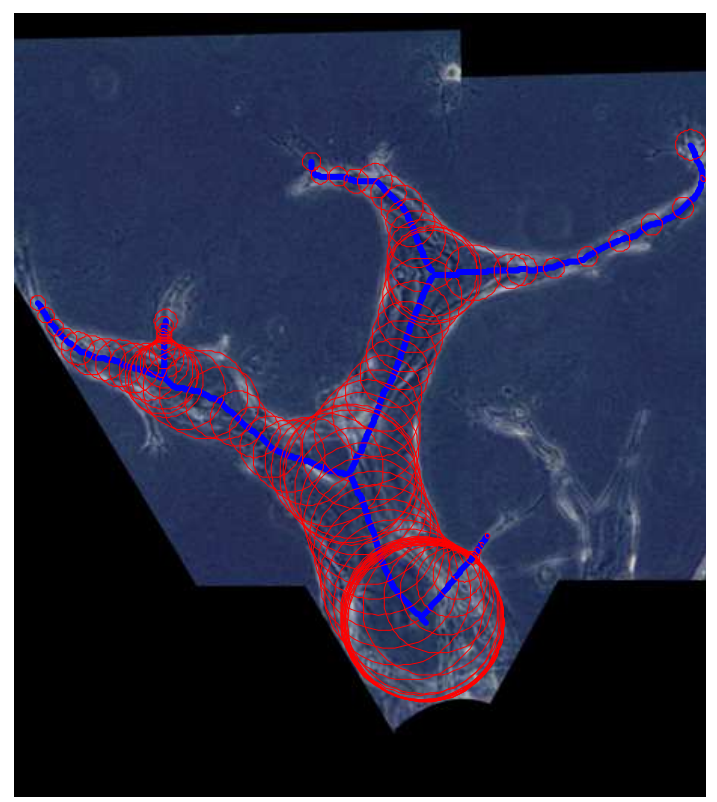

(f) Skeleton segments with radii.

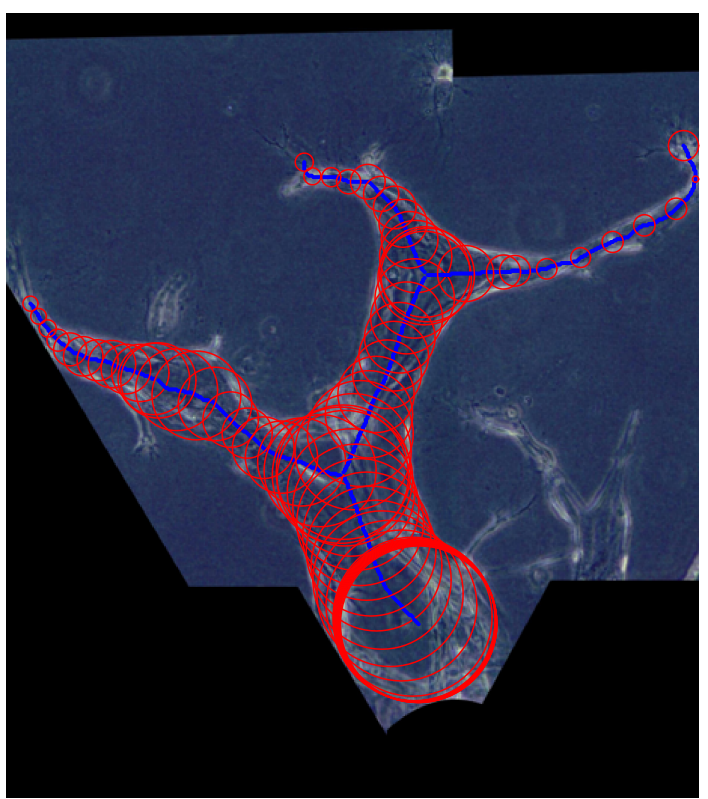
sification.

Figure 4.4: An example of skeletonization: (a) binary vessel shape; (b) boundary of the binary vessel; (c) skeletons from thinning; (d) skeletons from DT and AFMM; (e) skeleton segments with nodes; (f) skeleton segments with radii in the original image; (g) an example of a filopodia wrongly labeled as skeleton; and (h) skeleton segments with radii after SVM classification. 
rectangle is one part of the filopodia. In other words, the red node 3 in Fig. 4.4(g) is not a true branch node since it only connects with two skeleton segments that belong to a vessel. However, further increase of the threshold would lead to the loss of the main skeleton segments.

The number of branch nodes is important for estimating the number of branches. The critical feature for a branch node is that it has at least 3 connected skeleton segments that belong to a vessel. In order to decide whether a node is a branch node, we train a binary classifier, specifically, a binary SVM classifier, to remove the specious skeleton segments.

SVMs are a set of supervised learning methods used for classification, regression, and outlier detection [136]. Soft-margin SVM is applicable to classify linearly inseparable data by an optimal hyperplane, as shown in Fig. 4.5. It allows for a tolerable misclassification rate. The decision function for soft-margin SVM is given by [137]:

$$
D\left(\mathbf{x}^{i}\right)=w^{T} \mathbf{x}^{i}+b
$$

where $\mathbf{x}^{i}$ is the $i$ th template, $w$ is the normal vector of the hyperplane, and $b$ is a bias term.

To obtain the optimal hyperplane, we determine the values for $w$ and $b$ by solving

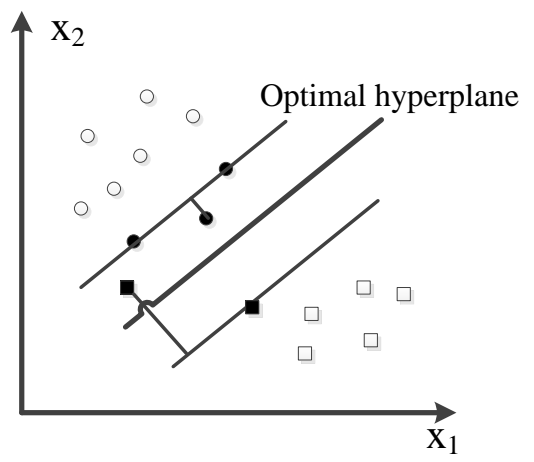

Figure 4.5: Diagram of support vector machine. 
the following optimization problems for $i=1, \cdots, N$ :

$$
\begin{gathered}
{[w, b]=\arg \min _{w, b}\left(\frac{|w|^{2}}{2}+C \sum_{i=1}^{N} \xi_{i}\right),} \\
\text { subject to } y^{i}\left(w^{T} \mathbf{x}^{i}+b\right) \geq 1-\xi_{i}, \quad \xi_{i} \geq 0,
\end{gathered}
$$

where $N$ is the number of the training templates, $y^{i}$ is the label of the $i$ th template, $\xi_{i}$ is the nonnegative slack variables, and $C$ is the margin parameter that determines the trade-off between the maximization of the margin and minimization of the classification error [138]. By introducing the nonnegative Lagrange multipliers $\alpha_{i}$ and $\beta_{i}$, we obtain:

$$
L=\frac{1}{2}|w|^{2}+C \sum_{i=1}^{N} \xi_{i}-\sum_{i=1}^{N} \alpha_{i}\left(y^{i}\left(w^{T} \mathbf{x}^{i}+b\right)-1+\xi_{i}\right)-\sum_{i=1}^{N} \beta_{i} \xi_{i}
$$

For $i=1, \cdots, N$, the following conditions must be satisfied for the optimal solution:

$$
\begin{gathered}
\frac{\partial L}{\partial w}=0, \\
\frac{\partial L}{\partial b}=0, \\
\frac{\partial L}{\partial \xi_{i}}=0, \\
\alpha_{i}\left(y^{i}\left(w^{T} \mathbf{x}^{i}+b\right)-1+\xi_{i}\right)=0, \\
\beta_{i} \xi_{i}=0, \\
\beta_{i} \geq 0 \quad \xi_{i} \geq 0 .
\end{gathered}
$$


Substituting equation (4.7) into equation (4.8) to (4.10) respectively, we obtain:

$$
\begin{gathered}
w=\sum_{i=1}^{N} \alpha_{i} y^{i} \mathbf{x}^{i}, \\
\sum_{i=1}^{N} \alpha_{i} y^{i}=0 \\
\alpha_{i}+\beta_{i}=C .
\end{gathered}
$$

After substituting equation (4.14) to (4.16) into equation (4.7), we obtain the optimum $\alpha_{i}$ as:

$$
\begin{array}{r}
\alpha_{i}=\arg \max _{\alpha_{i}}\left(\sum_{i=1}^{N} \alpha_{i}-\frac{1}{2} \sum_{i, j=1}^{N} \alpha_{i} \alpha_{j} y^{i} y^{j}\left(\mathbf{x}^{i}\right)^{T} \mathbf{x}^{j}\right), \\
\text { subject to } \sum_{i=1}^{N} \alpha_{i} y^{i}=0 \text { and } C \geq \alpha_{i} \geq 0,
\end{array}
$$

for $i=1, \cdots, N$. From equation (4.11), (4.12), and (4.16), we know there are three cases for $\alpha_{i}$ :

1. $\alpha_{i}=0: \xi_{i}=0$, and $\mathbf{x}^{i}$ is correctly classified.

2. $0<\alpha_{i}<C: \xi_{i}=0, y^{i}\left(w^{T} \mathbf{x}^{i}+b\right)=1$, and $\mathbf{x}^{i}$ is correctly classified and is called as an unbounded support vector.

3. $\alpha_{i}=C: \xi_{i} \geq 0, y^{i}\left(w^{T} \mathbf{x}^{i}+b\right)-1+\xi_{i}=0$, and $\mathbf{x}^{i}$ is called as bounded support vector. If $0 \leq \xi_{i}<1, \mathbf{x}^{i}$ is correctly classified, otherwise, $\mathbf{x}^{i}$ is misclassified.

The vector $w$ can be calculated after substituting the optimum values of $\alpha_{i}$ into 
equation (4.14), whereas $b$ can be calculated as:

$$
b=\frac{1}{\left|U_{s}\right|} \sum_{i \in U_{s}}\left(y^{i}-w^{T} \mathbf{x}^{i}\right)
$$

where $U_{s}$ is the set of unbounded support vector indices.

In order to train an SVM classifier, the first step is to prepare the training data. We preprocess the experimental phase contrast images to get the binary vessel shapes, and then apply DT and AFMM to extract the skeleton segments with radii. By comparing with their corresponding confocal images, each skeleton segment is labeled as positive training template or negative training template based on whether it belongs to a vessel or not.

Fig. 4.6 shows an example of how we obtain the training templates. The image in Fig. 4.6(a) is cropped from the experimental phase contrast image, and Fig. 4.6(b) is its corresponding confocal image. Fig. 4.6(c) shows the skeleton segments and nodes obtained from DT and AFMM. By comparing with Fig. 4.6(b), we label each skeleton segment in Fig. 4.6(c) as a positive or negative training template. The positive templates and negative templates are displayed in Fig. 4.6(d) and 4.6(e) respectively. For each skeleton segment $i$, its label $y^{i}$ is defined as:

$$
y^{i}=\left\{\begin{array}{cc}
1 & \text { if skeleton segment belongs to a vessel } \\
-1 & \text { otherwise }
\end{array}\right.
$$

The features we extract for each training template include:

- Segment length $l$ : a scalar value which measures the length of the skeleton segment,

- Segment radii $\mathbf{r}$ : a vector containing a set of radii acquired at the equidistant points in the skeleton segment,

- Maximum radius $r_{\max }$ : maximum value of the segment radii $\mathbf{r}$, 


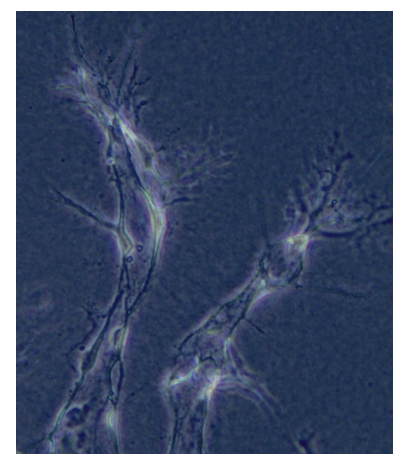

(a) Phase contrast image.
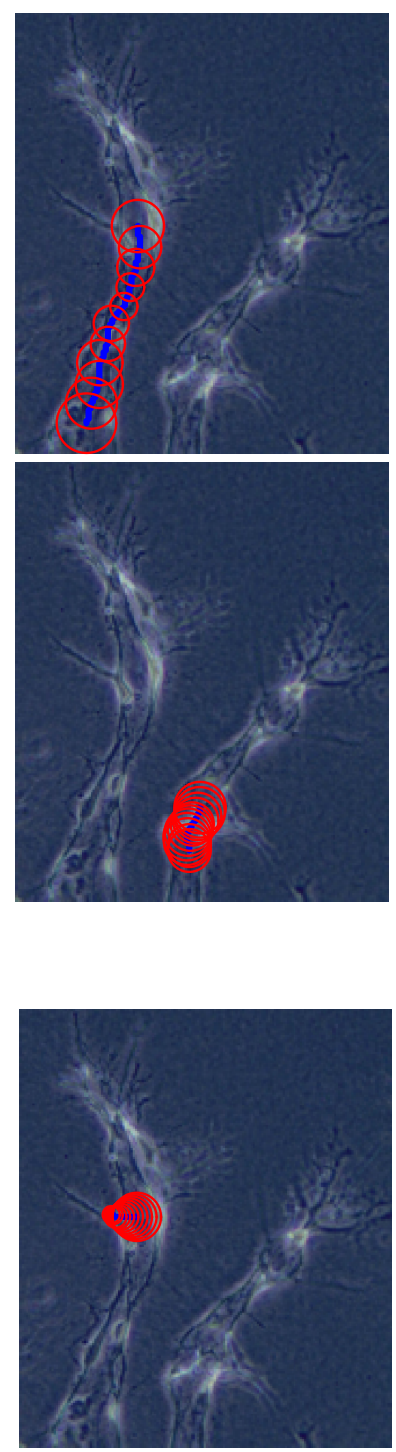

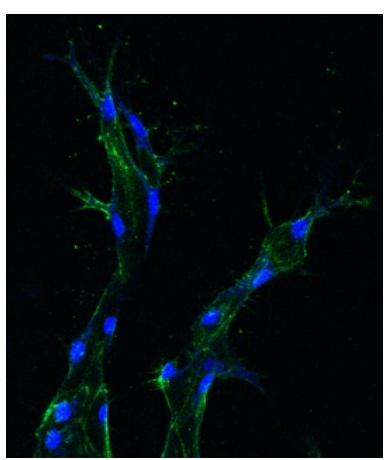

(b) Confocal image.
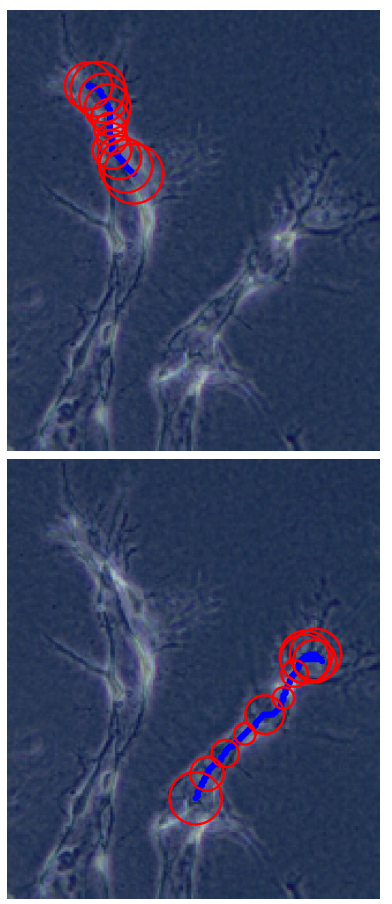

(d) Positive templates.

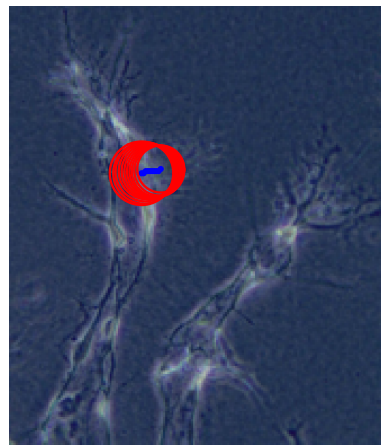

(e) Negative templates.

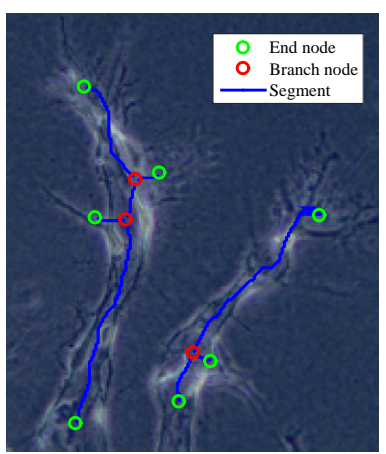

(c) Skeleton and nodes.
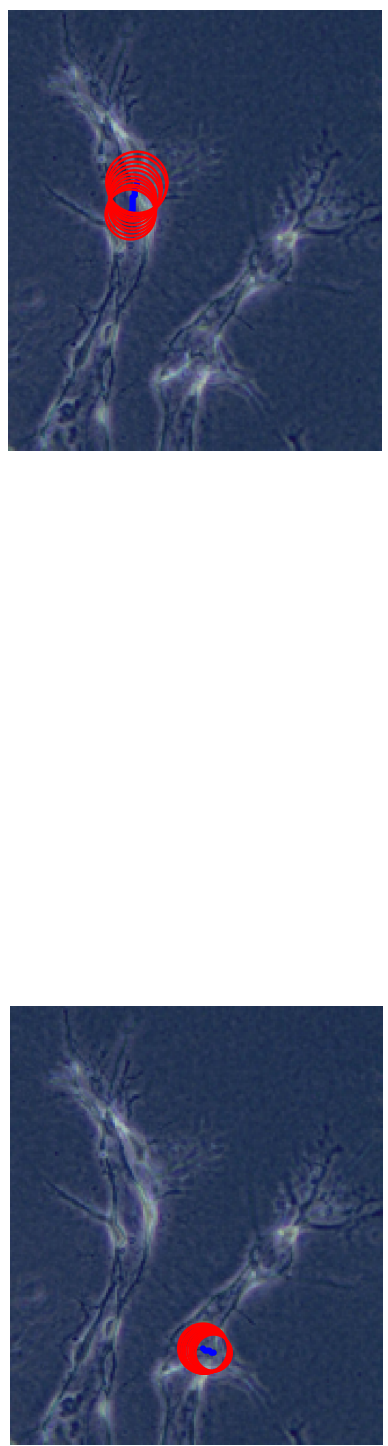

Figure 4.6: Examples of training templates for the binary SVM classifier: (a) and (b) are vessels from confocal image and phase contrast image respectively; (c) shows skeleton segments and nodes from DT and AFMM; (d) are positive templates; and (e) are negative templates.

NANYANG TEChNOLOGICAL University 
- Minimum radius $r_{m i n}$ : minimum value of the segment radii $\mathbf{r}$,

- Connection type $J$ : a scalar value based on the two nodes at the end of the skeleton segment which includes: branch-branch node connection $(J=1)$, branch-end node connection $(J=2)$, and end-end node connection $(J=3)$.

We aim to find the best combination of the above features to train the binary SVM classifier. Our training data includes 214 positive training samples and 102 negative training samples. After testing every possible combination of features, we obtain the most appropriate feature vector as follows:

$$
\mathbf{x}^{i}=\left[\begin{array}{c}
l^{i} \\
r_{\max }^{i} \\
r_{\min }^{i} \\
J^{i}
\end{array}\right]
$$

for the $i$ th training template. We obtain $84 \%$ accuracy in 5 -fold cross-validation when training the SVM classifier with the feature vector in equation (4.21).

We apply the trained SVM classifier to label and remove the non-vessel segments from the image. Fig. 4.4(h) shows the final skeleton with vessel radii after SVM classification.

For each node, the number of the segments it connects to is updated accordingly. The nodes whose updated number of connected segments is equal to or larger than three are labeled as the final branch nodes. The nodes with two connected segments are called junction nodes. 


\subsubsection{Branch Tracking}

\subsubsection{Base Nodes Determination}

We obtain all the skeleton segments with radii as explained in Section 4.2.1. However, it is not clear which segments are included in each individual branch. Consequently, to track the vessel formation, we first need to join the linked skeleton segments and identify the route of each branch.

As pointed out in Section 4.2.1, there are generally two scenarios for the skeletons, as illustrated in Fig. 4.7(a) and 4.7(b). Their generalized schematic diagrams are depicted in Fig 4.7(c) and 4.7(d) respectively. We define the base node as the node where a branch starts with. Generally, the branches originally from one same tip cell will share a same base node.

In Scenario 1, all the branches are originally from a same tip cell. This scenario is quite straightforward. The node which is nearest to the monolayer is selected as the base node, which is plotted in red in Fig. 4.7(e).

In Scenario 2, the vessels are originally from two different tip cells. However, they are connected by a segment (located in the green box in Fig. 4.7(b)) which is resulted from the artifact of skeletonization. In order to determine the base nodes, we need to detect and remove the skeleton segment which connects the two parts. The two base nodes are plotted in red in Fig. 4.7(f). Algorithm 2 demonstrates how we determine the scenario so as to find the base nodes.

Based on the observations of the experimental images, the angle between the monolayer and the artificial skeleton segment which connects two parts is small. To account for this angle, for a skeleton segment with node $\mathrm{M}$ and $\mathrm{N}$, the parameter ratio is estimated:

$$
\text { ratio }_{\mathrm{MN}}=\frac{\left|y_{N}-y_{M}\right|}{\left|x_{N}-x_{M}\right|}
$$




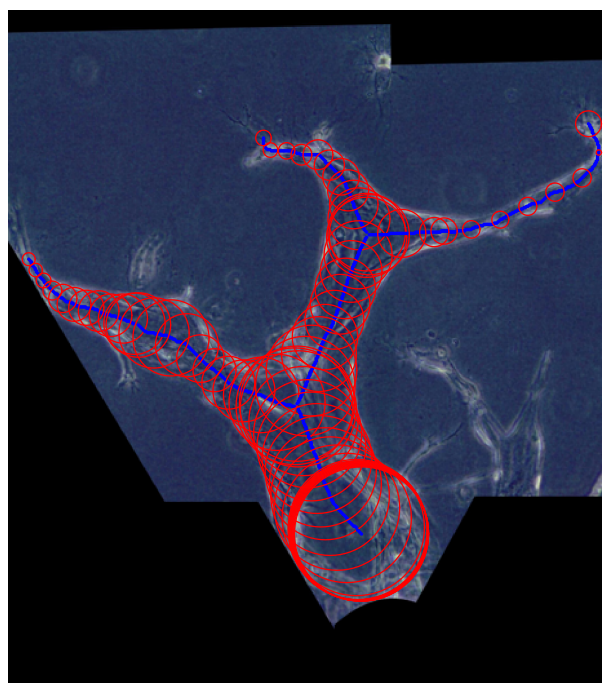

(a) Example of Scenario 1.

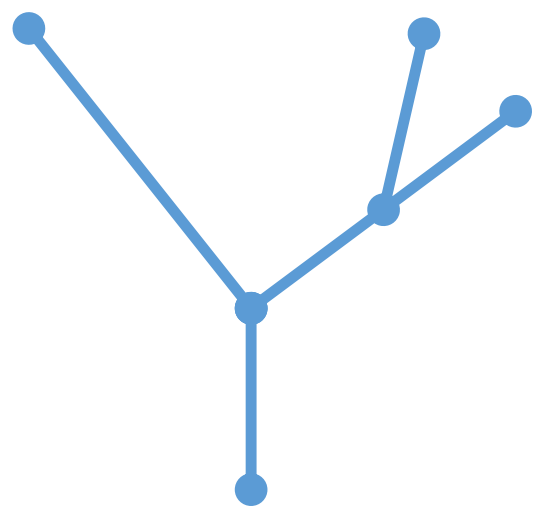

(c) Schematic diagram of Scenario 1.

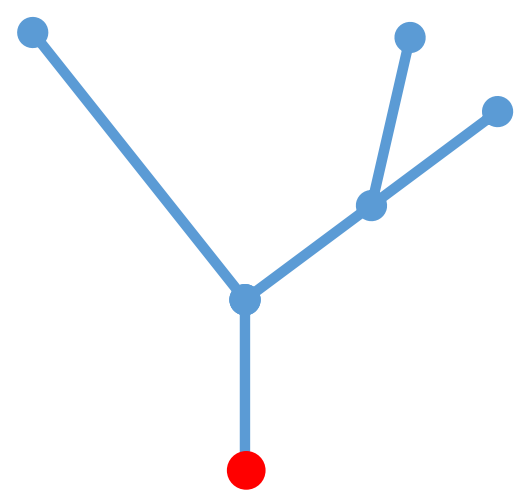

(e) Schematic diagram of Scenario 1: the red node is the base node.

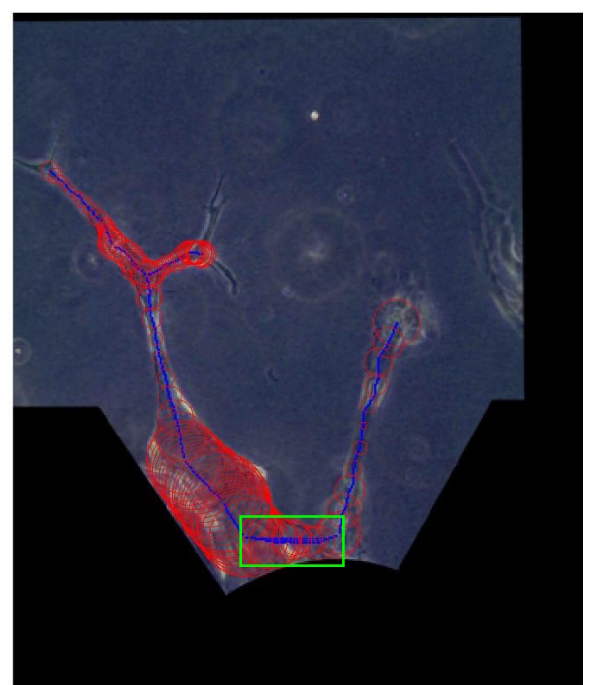

(b) Example of Scenario 2.

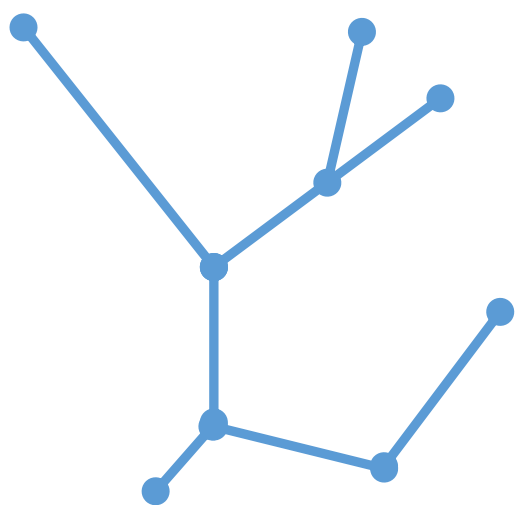

(d) Schematic diagram of Scenario 2.

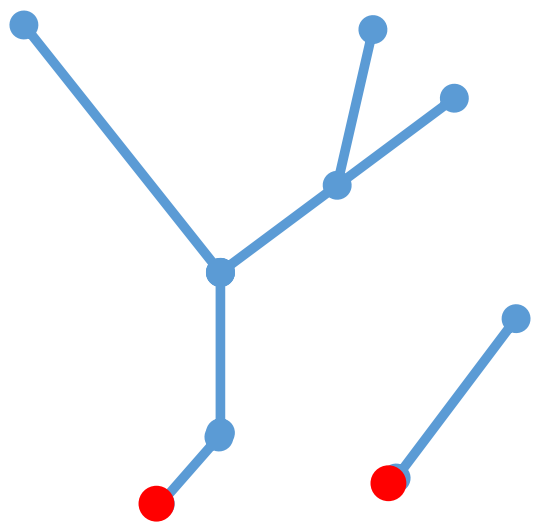

(f) Schematic diagram of Scenario 2: the red nodes are the base nodes.

Figure 4.7: Base nodes determination for the two different scenarios: (a) and (b) are the skeletons with radii in the experimental images for the two scenarios respectively; (c) and (d) are their corresponding generalized schematic diagrams; (e) and (f) indicate the based nodes in red. 


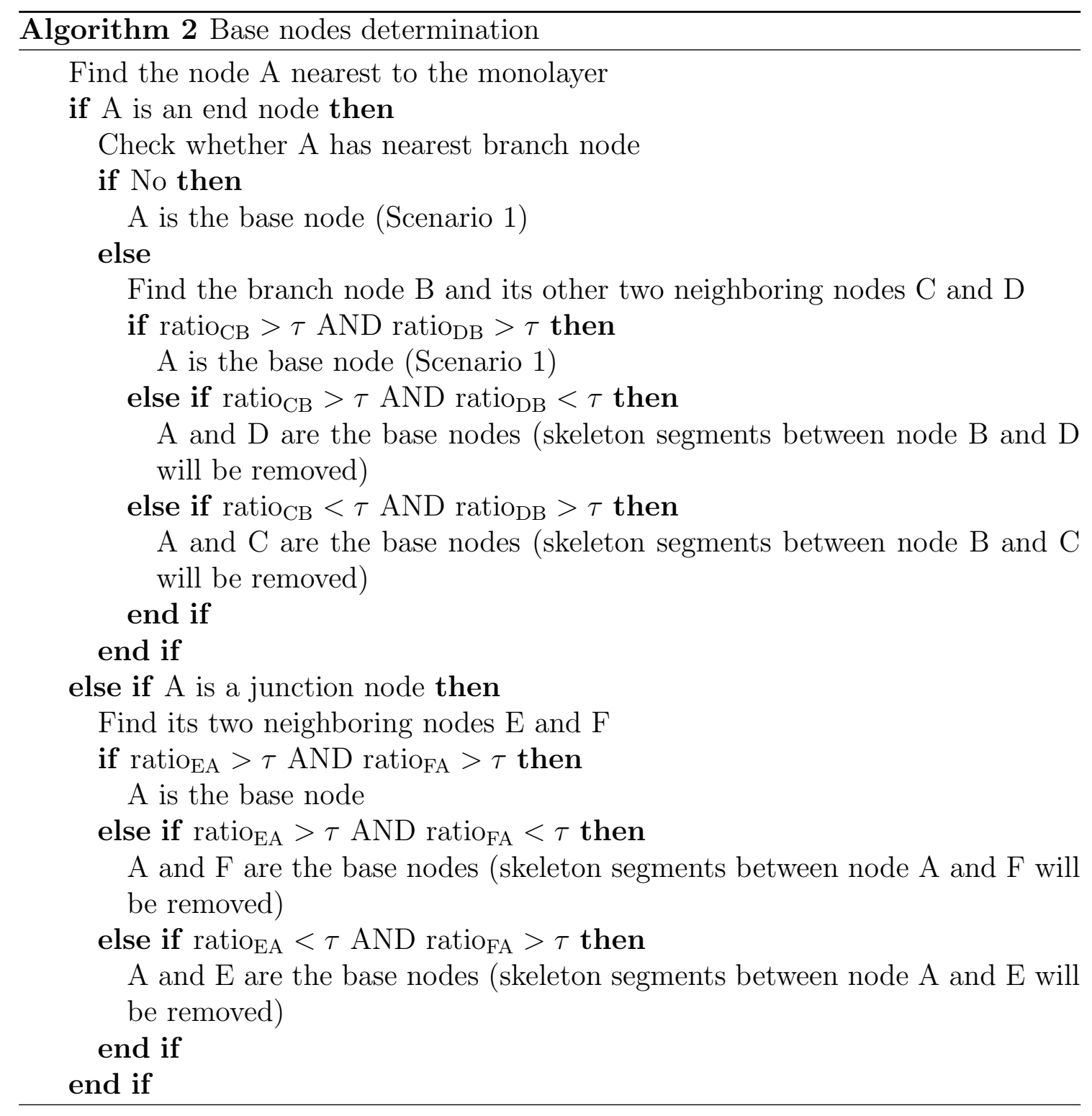


where $\left(x_{M}, y_{M}\right)$ and $\left(x_{N}, y_{N}\right)$ are the coordinates of node $\mathrm{M}$ and $\mathrm{N}$ respectively. The ratios in Algorithm 2 can be estimated by equation (4.22). By comparing the ratios with a predefined threshold $\tau$ and following Algorithm 2, the base nodes and the final skeleton segments are determined.

With the diagram in Fig. 4.8 as an example, after base nodes determination, we have:

- Base node: node 1 ,

- Branch nodes: node 2 and 4,

- End nodes: node 3, 5, and 6,

- Segments: [1 2], [2 3], [2 4], [4 5], and [4 6],

where each segment is represented by the two nodes it connects to.

Next, we identify the route for each branch. As introduced, each branch starts with a base node and ends with an end node. Matlab function "graphshortestpath" finds

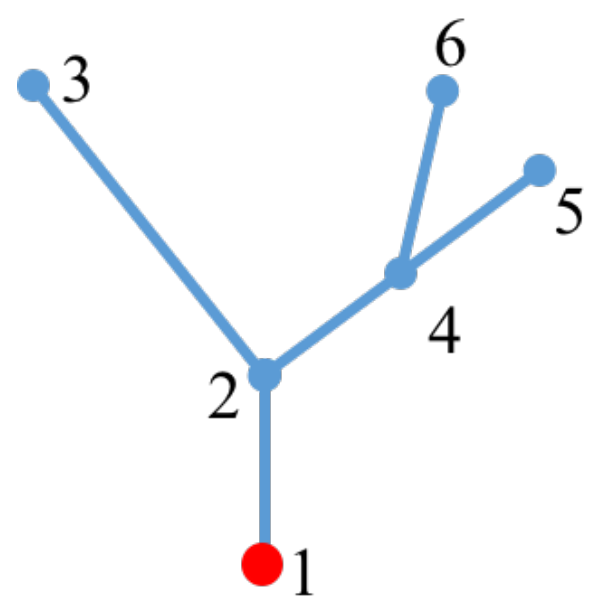

Figure 4.8: Diagram to illustrate the nodes and segments for identifying the route for each branch. 
the shortest path from one node to the other node in the graph [139]. By providing the base nodes, the end nodes, and the segments, we identify the routes for all the branches. The route for each branch is indicated by the nodes it passes by. For example, there are three branches in Fig. 4.8 and each of them is represented by:

- Branch 1: [1 2 3 3],

- Branch 2: [ $\left.\begin{array}{llll}1 & 2 & 4 & 6\end{array}\right]$,

- Branch 3: [1 $\left.\begin{array}{llll}1 & 2 & 4 & 5\end{array}\right]$.

Fig. 4.9(a) and Fig. 4.10(a) show the identified branch routes for the examples in Fig. 4.7(a) and 4.7(b) respectively, where each color represents one branch. Specifically, each branch of the vessels in Fig. 4.9(a) is plotted in Fig. 4.9(b) to 4.9(d), and each branch of the vessels in Fig. 4.10(a) is plotted in Fig. 4.10(b) to 4.10(d), where the blue lines are the continuous skeletons and the red circles are the branch radii at selected points in the skeletons.

As illustrated in Fig. 4.9 and Fig. 4.10, the quantitative values of the vessel length/width and number of branches for the experimental images are obtained.

\subsubsection{Branch Association Over Time}

After identifying the route for each branch, the next step is to track the branch formation over time. All the branches from a same tip cell that migrated from the monolayer share a same base node. All the branches sharing a same base node are defined as a vessel part. Fig. 4.11 shows the skeletons of the angiogenic vessels over two consecutive days. Each image in Fig. 4.11 contains two vessel parts, which are within the red rectangular box and yellow rectangular box respectively. Over time sequence, the vessel part within the red box are from same tip cell (i.e. cell 1) and the vessel part within the yellow box are from same tip cell (i.e. cell 2). We firstly 


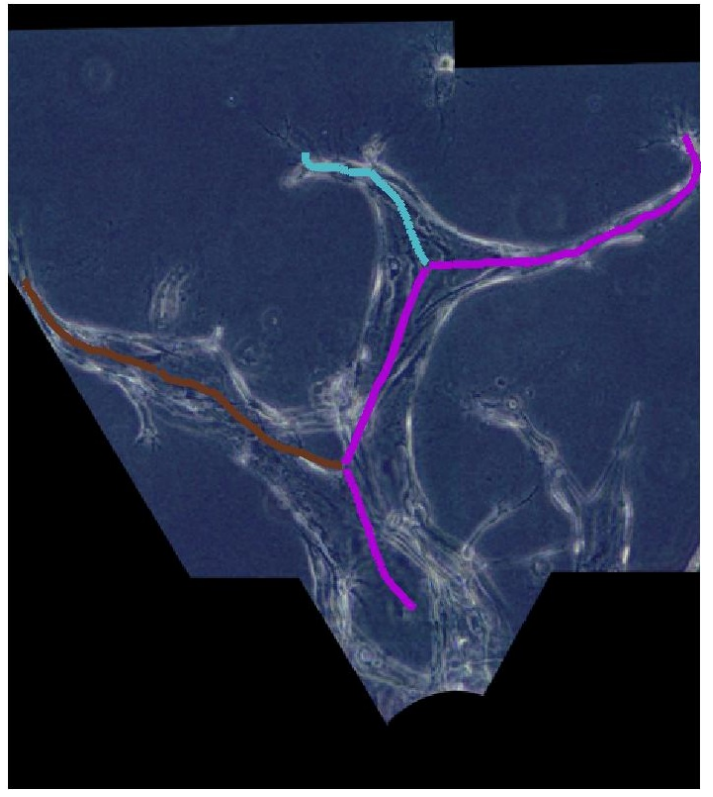

(a) Identified routes for all the branches.

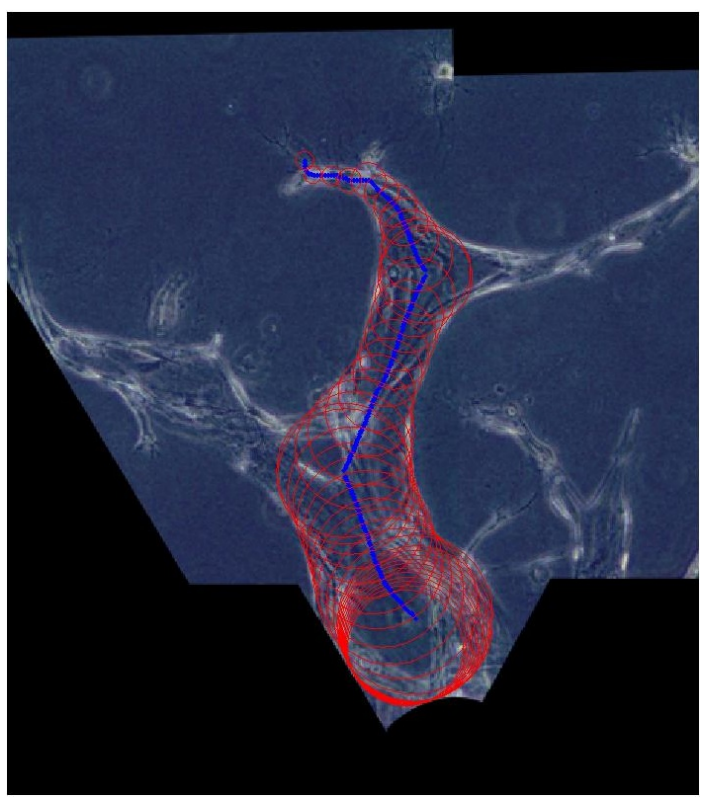

(c) Branch 2.

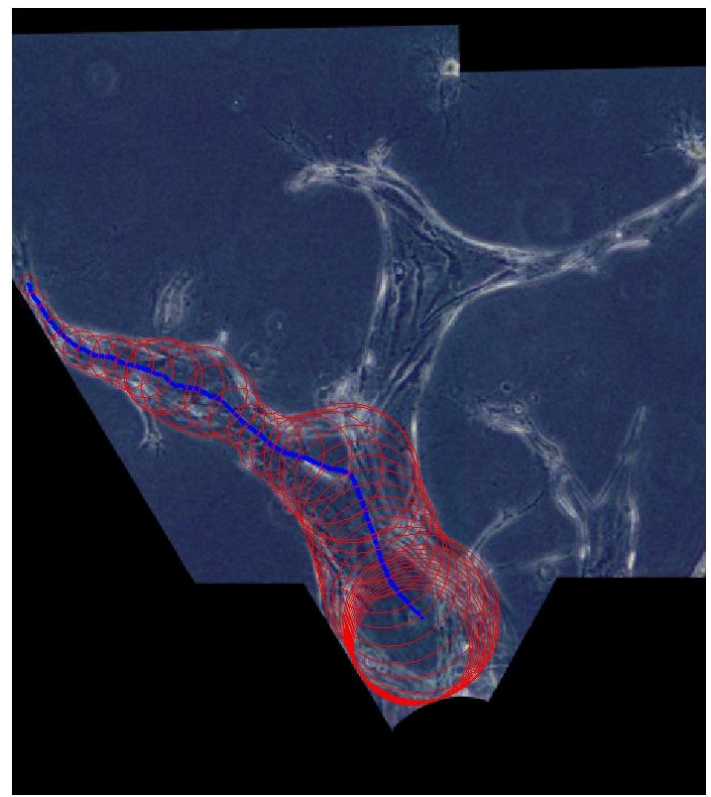

(b) Branch 1.

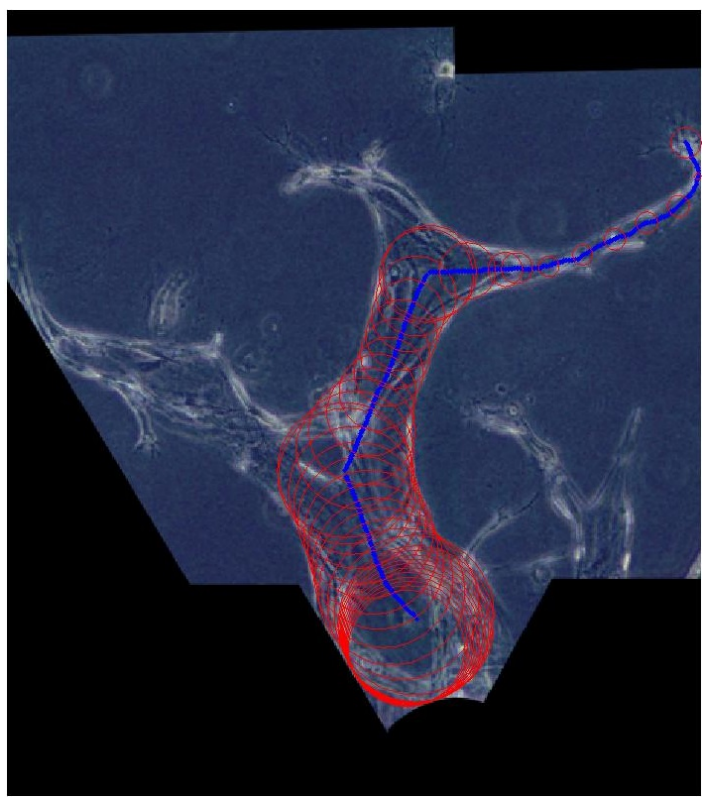

(d) Branch 3 .

Figure 4.9: Identified branch routes for the example in Fig. 4.7(a): each branch of the vessels is plotted in (b) to (d), where the blue lines are the continuous skeletons and the red circles are the branch radii at selected points in the skeletons. 


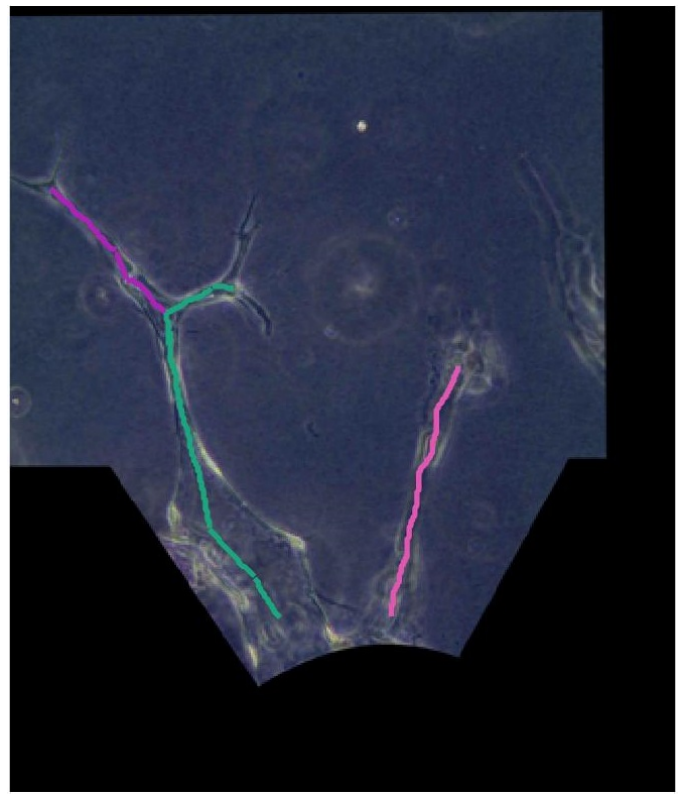

(a) Identified routes for all the branches.

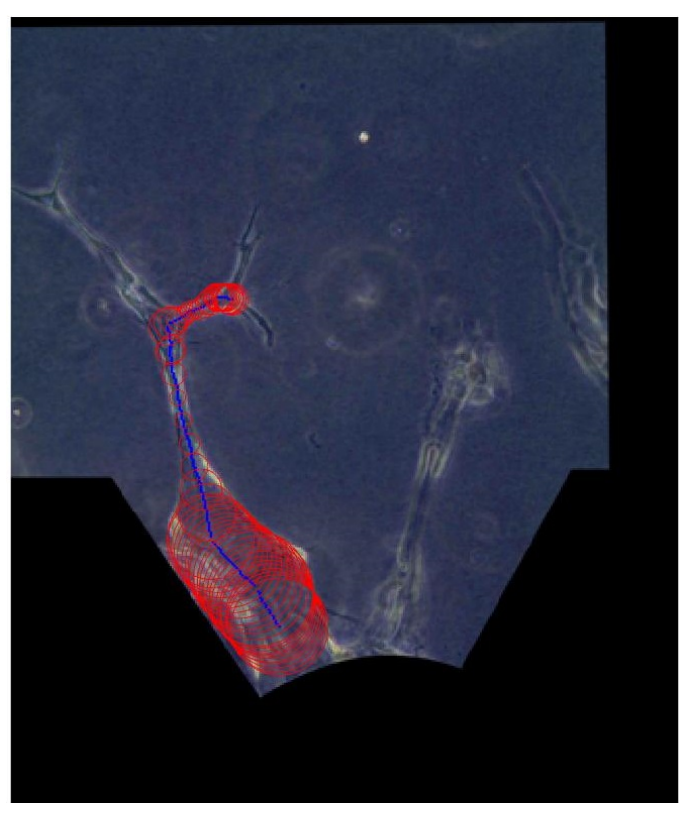

(c) Branch 2 .

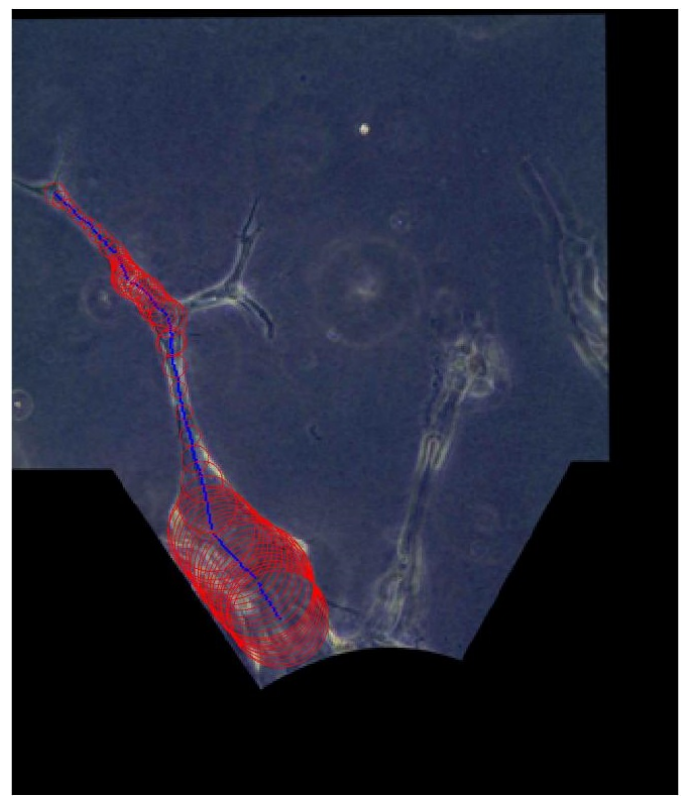

(b) Branch 1 .

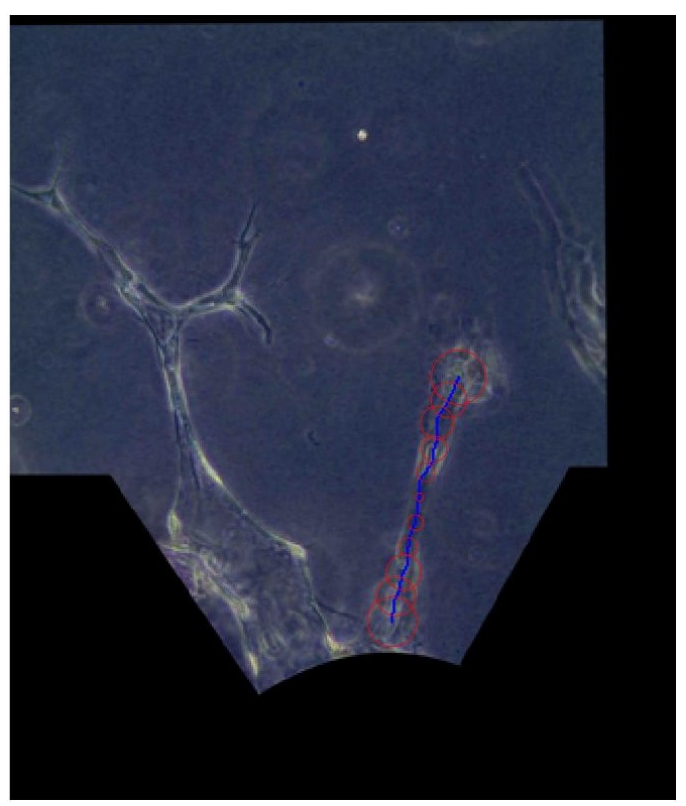

(d) Branch 3.

Figure 4.10: Identified branch routes for the example in Fig. 4.7(b): each branch of the vessels is plotted in (b) to (d), where the blue lines are the continuous skeletons and the red circles are the branch radii at selected points in the skeletons. 
associate the vessel part as a whole and then associate branches in each vessel part respectively over time.
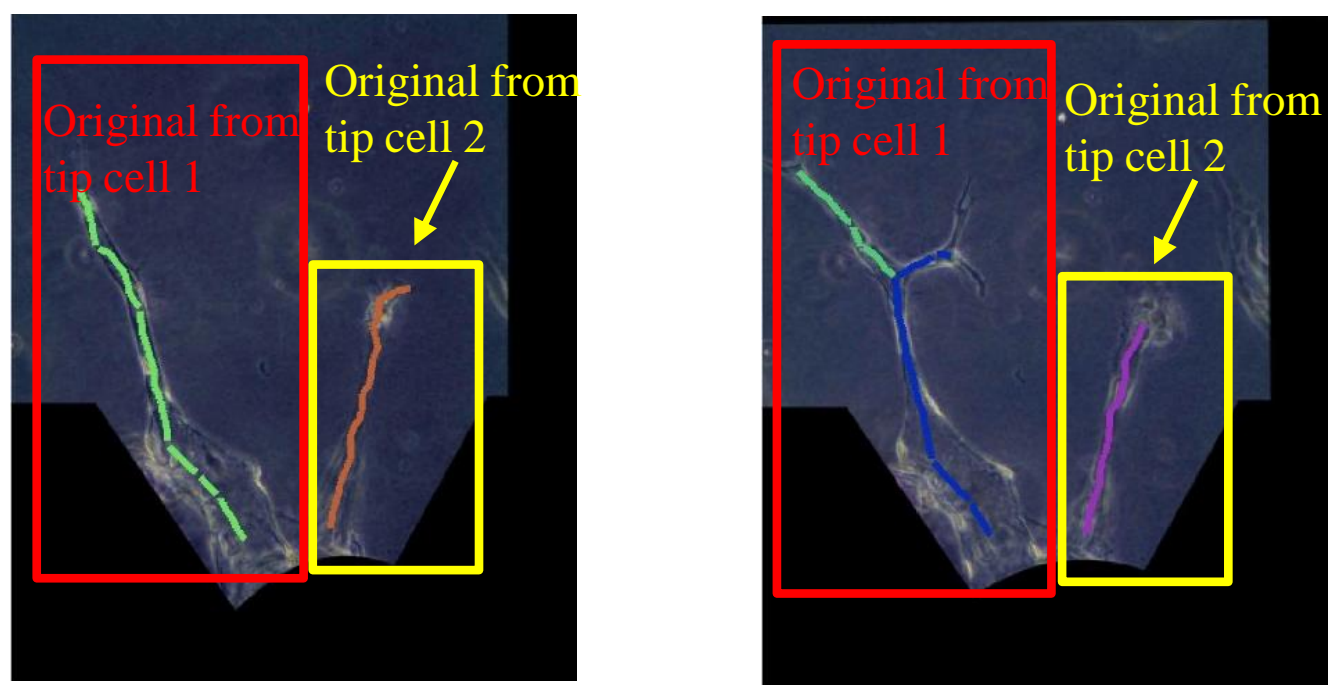

Figure 4.11: Skeletons of the angiogenic vessels over two consecutive days: the vessels in the red rectangular box and in the yellow box are originally from two different tip cells that migrated from the monolayer.

\section{Step 1: Associate vessel parts over time}

Each vessel part is regarded as a whole when matching the vessel parts over time. From the experimental observations, two different vessel parts generally do not overlap each other. In other words, for different vessel parts, their skeletons differ significantly in the average $x$-positions. Therefore, we associate the vessel parts by comparing their average $x$-positions with the following steps:

- Compute the number of vessel parts in frame $t+1$,

- Check whether there are new vessel parts by comparing with the number of the vessel parts in the previous frame $t$,

- Match the existing vessel parts and determine the new vessel parts based on their average $x$-positions by the "nearest-neighborhood" algorithm. 


\section{Step 2: Associate branches in each vessel part over time}

After associating the vessel parts, we match the branches in each associated vessel part over time. We start with tracking the branch nodes based on their relative locations. Suppose we have $n_{1}$ branch nodes in one vessel part at frame $t$ and $n_{2}$ branch nodes in its corresponding vessel part at frame $t+1$. The pairwise Euclidean distance matrix for the branch nodes in both vessel parts $\mathbf{C} \in \mathbb{R}^{n_{1} \times n_{2}}$ is defined as:

$$
\mathbf{C}=\left[\begin{array}{ccc}
c_{1,1} & \cdots & c_{1, n_{2}} \\
\vdots & \ddots & \vdots \\
c_{n_{1}, 1} & \cdots & c_{n_{1}, n_{2}}
\end{array}\right]
$$

$\mathbf{C}$ is a cost matrix to distinguish the new branch nodes and match the existing branch nodes.

The Hungarian method, a combinatorial optimization algorithm proposed by [140], is used to associate the branch nodes. However, as explained in Theorem 4.1 and Theorem 4.2, it is only applicable to a square matrix. For our case, the number of branch nodes changes over time when new branches form or existing branches retract. In other words, $\mathbf{C}$ is not a square matrix when $n_{2} \neq n_{1}$. Since the retraction is rarely observed in our experiments, we assume that the number of branch nodes changes only when new branches form. Specifically, only $n_{2} \geq n_{1}$ is considered.

We create a new square matrix $\mathbf{P} \in \mathbb{R}^{n_{2} \times n_{2}}$. If $n_{2}>n_{1}, \mathbf{P}$ is obtained by adding $n_{2}-n_{1}$ rows to $\mathbf{C}$ :

$$
p_{i, j}=\left\{\begin{array}{lr}
c_{i, j} & \text { for } i=1: n_{1} \text { and } j=1: n_{2} \\
c_{0} & \text { for } i=n_{1}+1: n_{2} \text { and } j=1: n_{2}
\end{array}\right.
$$

where $c_{0}$ is an assigned constant value that is larger than all the other distances. If $n_{2}=n_{1}$,

$$
p_{i, j}=c_{i, j} \quad \text { for } \quad i=1: n_{2} \text { and } j=1: n_{2} \text {. }
$$


We introduce an $n_{2} \times n_{2}$ matrix $\mathbf{Q}$ to optimize the matching of the branch nodes by minimizing:

$$
\sum_{i=1}^{n_{2}} \sum_{j=1}^{n_{2}} p_{i, j} q_{i, j}
$$

subject to the constraints:

$$
\begin{aligned}
& \sum_{i=1}^{n_{2}} q_{i, j}=1, \text { for } j=1, \cdots, n_{2}, \\
& \sum_{j=1}^{n_{2}} q_{i, j}=1, \text { for } i=1, \cdots, n_{2} .
\end{aligned}
$$

The conditions in equation (4.27) and (4.28) enforce that all elements in $\mathbf{Q}$ are zeros except for one 1 in each row and column, guaranteeing a one-to-one matching.

The Hungarian method is rooted in the following two Theorems. Through Theorem 4.1, the matrix $\mathbf{P}$ is transformed into another matrix $\hat{\mathbf{P}}$, which contains an independent set of $n_{2}$ zeros. Theorem 4.2 generates the optimal solution $\mathbf{Q}$ for the association [97, 141, 142].

Theorem 4.1. A solution $\mathrm{Q}$ minimizes

$$
\sum_{i=1}^{n_{2}} \sum_{j=1}^{n_{2}} p_{i, j} q_{i, j}
$$

if and only if it minimizes

$$
\sum_{i=1}^{n_{2}} \sum_{j=1}^{n_{2}} \hat{p}_{i, j} q_{i, j}
$$

where $\hat{p}_{i, j}=p_{i, j}-u_{i}-v_{j}, u_{i}$ and $v_{i}$ are real numbers for all $i=1, \cdots, n_{2}$ and $j=1, \cdots, n_{2}$.

Theorem 4.2. If all the elements in $\mathbf{P}$ satisfy $p_{i, j} \geq 0$ and $\left\{p_{1, j_{1}}, p_{2, j_{2}}, \cdots, p_{n_{2}, j_{n_{2}}}\right\}$ is an independent set of $n_{2}$ zeros in $\mathbf{P}$, then the matrix $\mathbf{Q}$ with

$$
\begin{aligned}
& q_{1, j_{1}}=1, \\
& q_{2, j_{2}}=1,
\end{aligned}
$$




$$
\begin{aligned}
q_{n_{2}, j_{n_{2}}} & =1 \\
q_{i, j} & =0 \text { for all other } i \text { and } j,
\end{aligned}
$$

is the optimal solution to minimize $\sum_{i=1}^{n_{2}} \sum_{j=1}^{n_{2}} p_{i, j} q_{i, j}$.

We determine the new branch nodes and associate the existing branch nodes from the optimal solution $\mathbf{Q}$. For two consecutive frames $t$ and $t+1$, the elements $q_{i, j}=1$ in $\mathbf{Q}$ indicates that at frame $t+1$ :

$$
\begin{cases}j \text { is a new branch node, } & \text { if } p_{i, j}=c_{0} \\ j \text { corresponds to } i \text { at frame } t, & \text { otherwise. }\end{cases}
$$

For the case that branch node $j$ at frame $t+1$ corresponds to branch node $i$ at frame t, we match the segments connect to $j$ and $i$ based on their average $x$-positions. For the case that $j$ is a new branch node, we label the skeleton segments connected to $j$ as new branches.

We use the examples in Fig. 4.12 to demonstrate the segment association. Table. 4.1 lists the the route for each branch in Fig. 4.12.

Table 4.1: Branches for the vessels in Fig. 4.12

\begin{tabular}{|l|l|}
\hline & Branches \\
\hline Day 9 & {$\left[\begin{array}{lll}1 & 2 & 3\end{array}\right],\left[\begin{array}{lll}1 & 2 & 4\end{array}\right]$} \\
\hline Day 10 & {$\left[\begin{array}{llll}1 & 2 & 3\end{array}\right],\left[\begin{array}{llll}1 & 2 & 4\end{array}\right],\left[\begin{array}{llll}1 & 2 & 4\end{array}\right]$} \\
\hline
\end{tabular}

As shown in Fig. 4.12, branch node 2 in day 9 corresponds to branch node 2 in day 10. The segments connected to the branch node 2 in day 9 are [2 3$]$ and [2 4]. The segments connected to the branch node 2 in day 10 are [2 3] and [2 4]. By comparing the average $x$-positions of these segments, we obtain that segment [2 3$]$ in day 9 corresponds to [2 3 ] in day 10, and segment [2 4 ] in day 9 corresponds to [2 4 ] in day 10. For the new branch node 4 in day 10, the segments [4 5] and [4 6] 


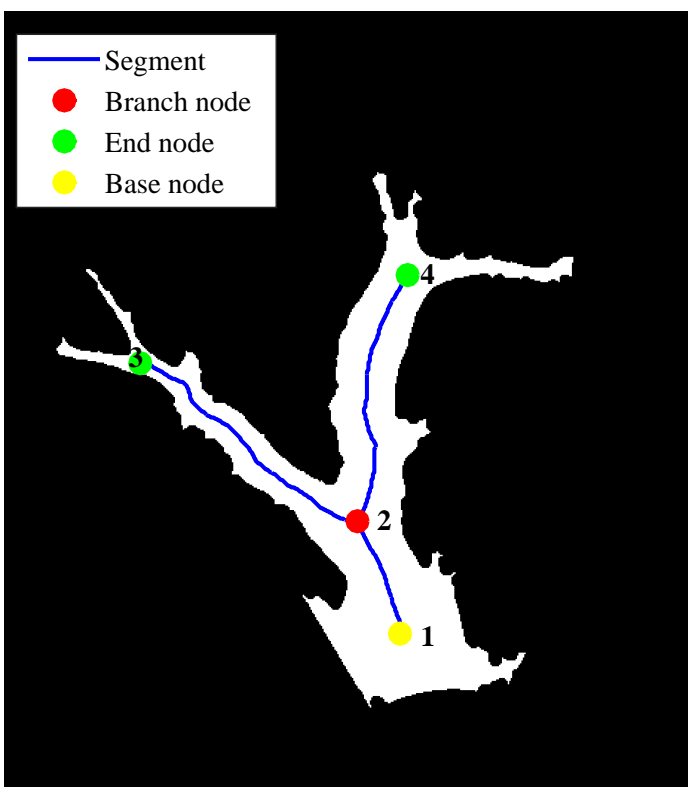

(a) Day 9 .

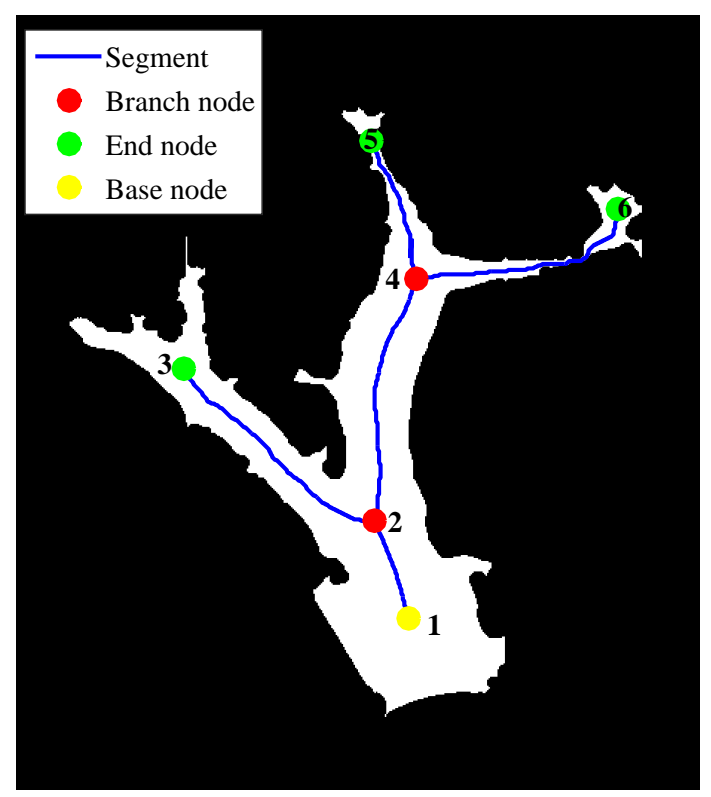

(b) Day 10 .

Figure 4.12: Skeletons and nodes for angiogenic vessels of two consecutive days.

connected to it are labeled as new branches.

Table. 4.2 illustrates the results of the branch formation tracking, specifically, for the examples in Fig. 4.12. More generally, this table shows the historical information for each branch, from the first day till last day of the experiment.

Table 4.2: Examples of the branch formation tracking results.

\begin{tabular}{|c|l|c|c|c|}
\hline Day 1 & $\cdots$ & Day 9 & Day 10 & Branch \\
\hline \multirow{3}{*}{ No vessels } & $\cdots$ & {$\left[\begin{array}{lll}1 & 2 & 3\end{array}\right]$} & {$\left[\begin{array}{lll}1 & 2 & 3\end{array}\right]$} & $\mathbf{1}$ \\
\cline { 2 - 6 } & \multirow{2}{*}[\begin{array}{lll}{1}&{2}&{4}\end{array}]{} & {$\left[\begin{array}{llll}1 & 2 & 4 & 5\end{array}\right]$} & $\mathbf{2}$ \\
\cline { 4 - 6 } & & {$\left[\begin{array}{lll}1 & 2 & 4\end{array}\right]$} & $\mathbf{3}$ \\
\hline
\end{tabular}




\subsection{Results and Discussion}

\subsubsection{Tracking of Vessel Formation}

By applying the AVFTS introduced in Section 4.2, we are able to generate the historical map for each branch, as illustrated in Table. 4.2. Meanwhile, we obtain the length and width of the angiogenic vessels based on the coordinates and the radii of the skeleton segments.

Fig. 4.13 and Fig. 4.14 illustrate the branch formation tracking results. The angiogenic vessels within a sprouting region are shown over different days (from day 5 to day 9). When new branch node appears, the newly formed branch is represented by a new centerline with a new color (indicated in Fig. 4.13 and Fig. 4.14). We tested the AVFTS on 20 sprouting regions, each of which contains 5 to 6 frames. By comparing with the ground truth obtained from manual tracking by visual inspection, the precision is estimated as $97.3 \%$ and the recall is estimated as $93.9 \%$.

\subsubsection{Application of the AVFTS}

The proposed AVFTS system in Section 4.2 provides us branch information including the length and width of each branch, and the number of branches for each sprouting region. By combining such branch information with the cell locations obtained from Section 3.2, cell phenotypes can be estimated as we will explain in Section 4.3.2.1. Biologists can also apply this proposed AVFTS system on the microscopy images obtained under different experimental conditions when they study the influence of different angiogenic factors on angiogenic vessel morphologies. As an illustration, we provide quantitative comparisons of the angiogenic vessels formed under different S1P conditions in Section 4.3.2.2. 


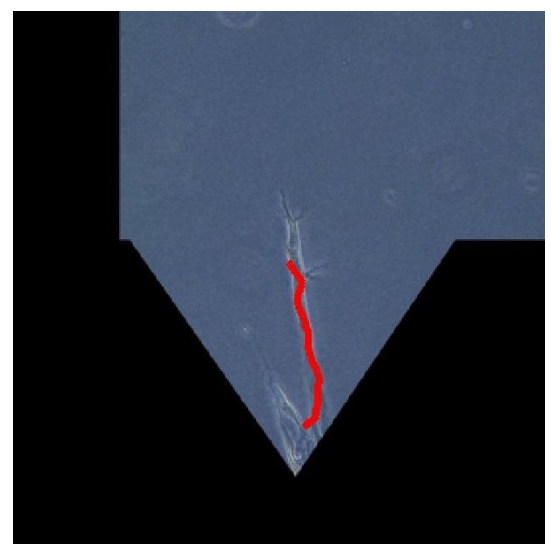

(a) Day 5 .

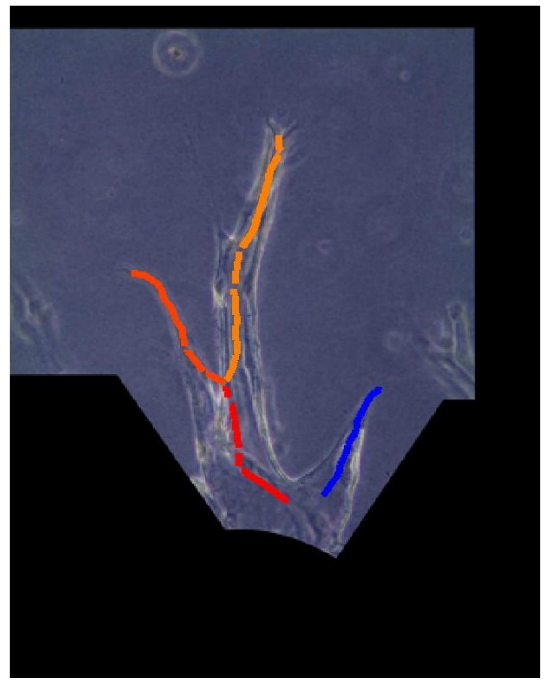

(c) Day 7 .

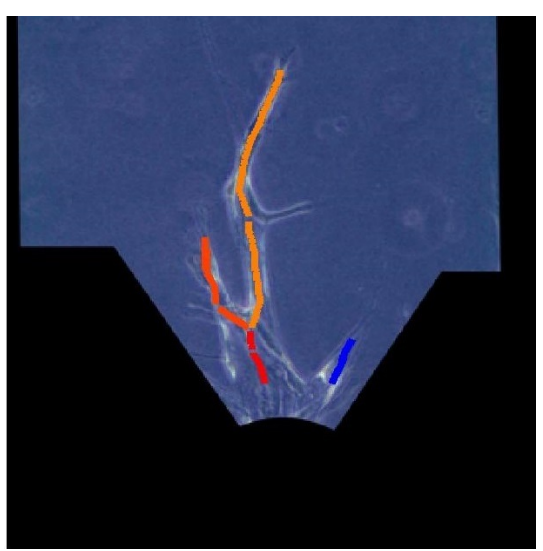

(b) Day 6 .

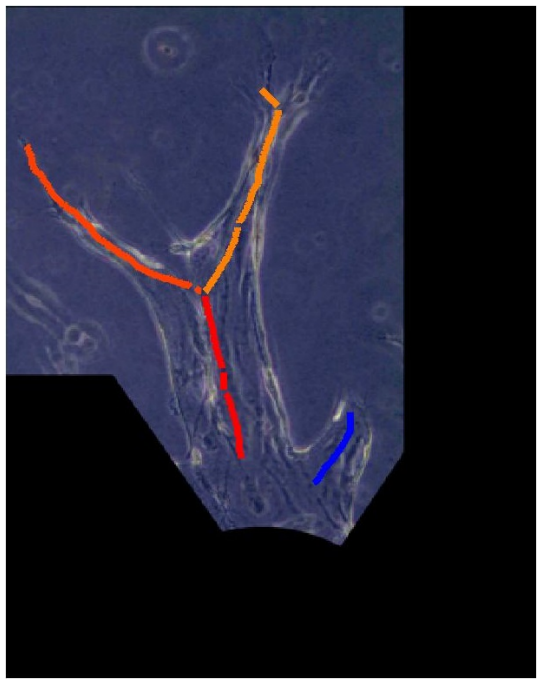

(d) Day 8 .

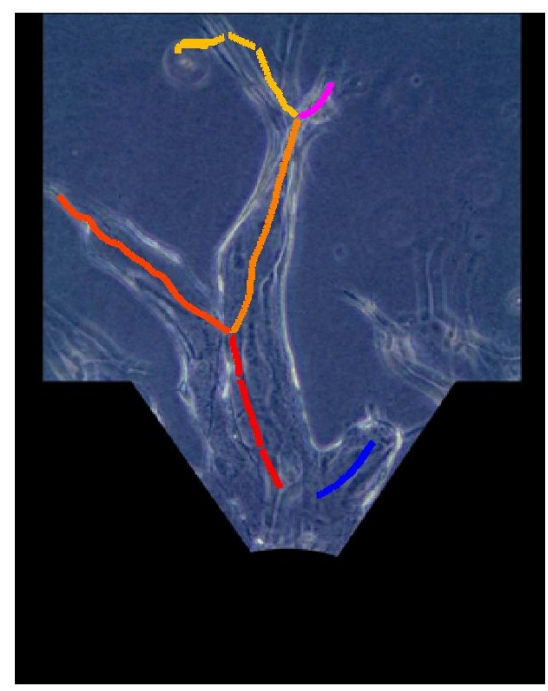

(e) Day 9.

Figure 4.13: Example 1 for branch tracking of angiogenic vessels within a sprouting region from day 5 to day 9 . 


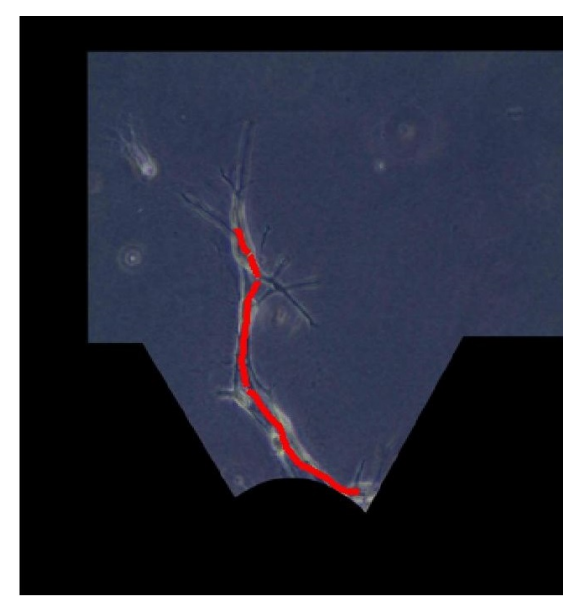

(a) Day 5 .

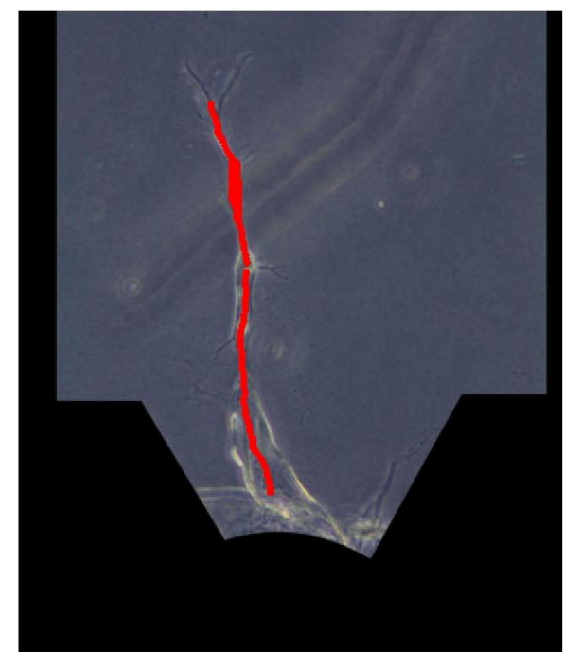

(c) Day 7.

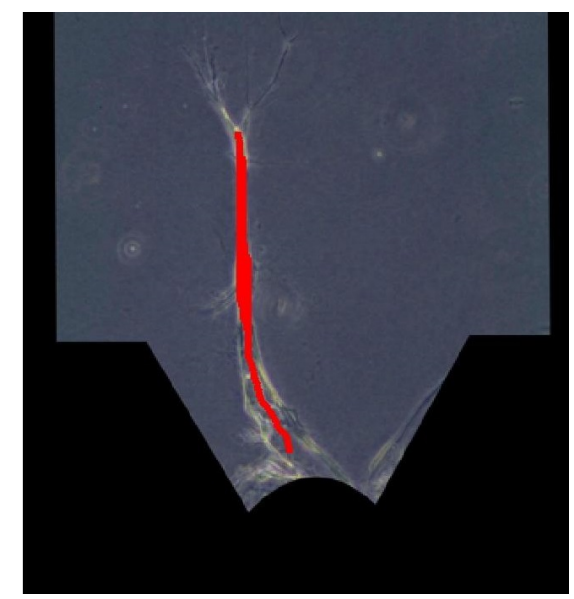

(b) Day 6 .

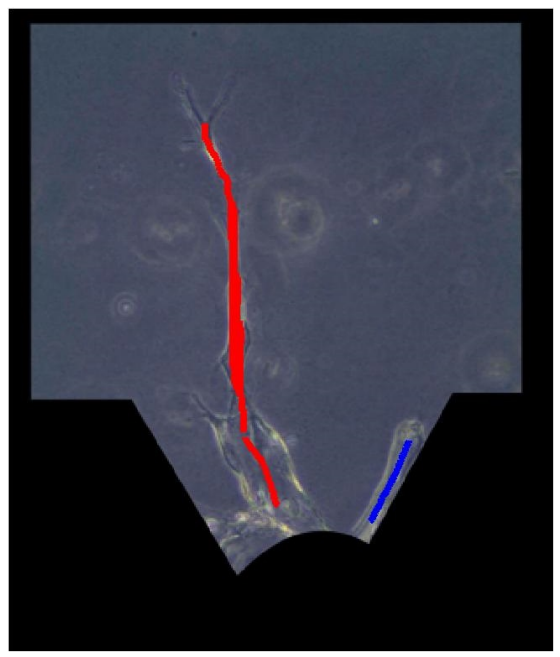

(d) Day 8 .

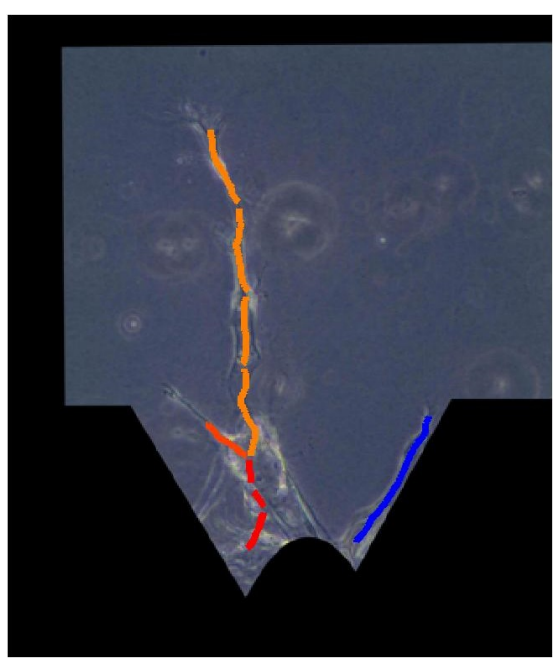

(e) Day 9 .

Figure 4.14: Example 2 for branch tracking of angiogenic vessels within a sprouting region from day 5 to day 9 . 


\subsubsection{Cell Phenotype Identification}

As introduced in Section 1.1, the dynamic competition of cell phenotypes serves an important function for angiogenic vessel formation [15]. Identification of tip and stalk cell phenotypes is important for investigating their different behaviors and unraveling the mechanisms for their inter-transition [143, 144].

Earlier studies have shown that tip cells are at the leading position in the branches in order to sense the stimuli and lead the way $[4,52,66,145]$. Since we obtained the cell locations in Section 3.2 and branch information in Section 4.2, the phenotype for each cell can be identified by considering its relative location in the branch.

In Fig. 4.16(a), we provide an example of angiogenic vessels with both cell and branch information. The cell locations, branch skeleton, and the radii of the vessels are plotted as green stars, blue lines, and red circles respectively.

As is well known, each branch is headed by a tip cell [4]. In other words, the number of tip cells equals to the number of branches in the angiogenic vessels in a sprouting region. For instance, the angiogenic vessels in Fig. 4.16(a) have 3 branches, and each of them has one tip cell.

As explained, each branch is indicated by the nodes it passes. For instance, branch

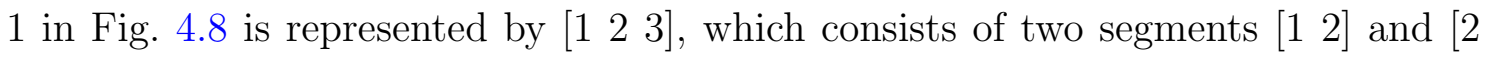
3]. Since tip cells lead the way, they are expected to be located in the last skeleton segment farthest away from the monolayer, e.g. segment [2 3] for branch 1 in Fig. 4.8. As a consequence, we can concentrate on the last skeleton segment in a branch to identify its corresponding tip cell.

Fig. 4.15(a) illustrates the diagram of the last segment in a branch with start point (black circle), end point (red circle), and the skeleton coordinates (black curved line). For each branch, our first step is to determine the tip cell candidates by selecting the $N_{\text {tip }}$ cells closest to the end point of the last segment. Based on the 
observations, tip cell and its two neighboring stalk cells are the closest cells to the end point. Thus, $N_{\text {tip }}=3$ is used in this chapter.

The blue circles in Fig. 4.15 indicate the three selected tip cell candidates. As the distances are about the same, we are unable to determine which cell is the tip cell based on the distances alone. Since the tip cell is always at the leading position in the branch, it can be identified as the cell that migrates the farthest along the direction of the segment. The projection of one tip cell candidate $i$ on the segment direction (blue dotted line with arrow in Fig 4.15(b)) is estimated as:

$$
p_{i}=\frac{\mathbf{v}_{s e} \cdot \mathbf{v}_{s i}}{\left|\mathbf{v}_{s e}\right|}
$$

where $\mathbf{v}_{s e}$ is the segment direction and $\mathbf{v}_{s i}$ is the vector between the start point and tip cell candidate $i$ :

$$
\begin{aligned}
& \mathbf{v}_{s e}=\mathbf{x}_{e}-\mathbf{x}_{s}, \\
& \mathbf{v}_{s i}=\mathbf{x}_{i}-\mathbf{x}_{s},
\end{aligned}
$$

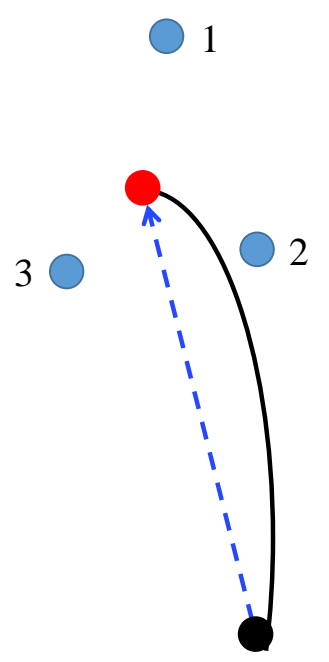

(a)

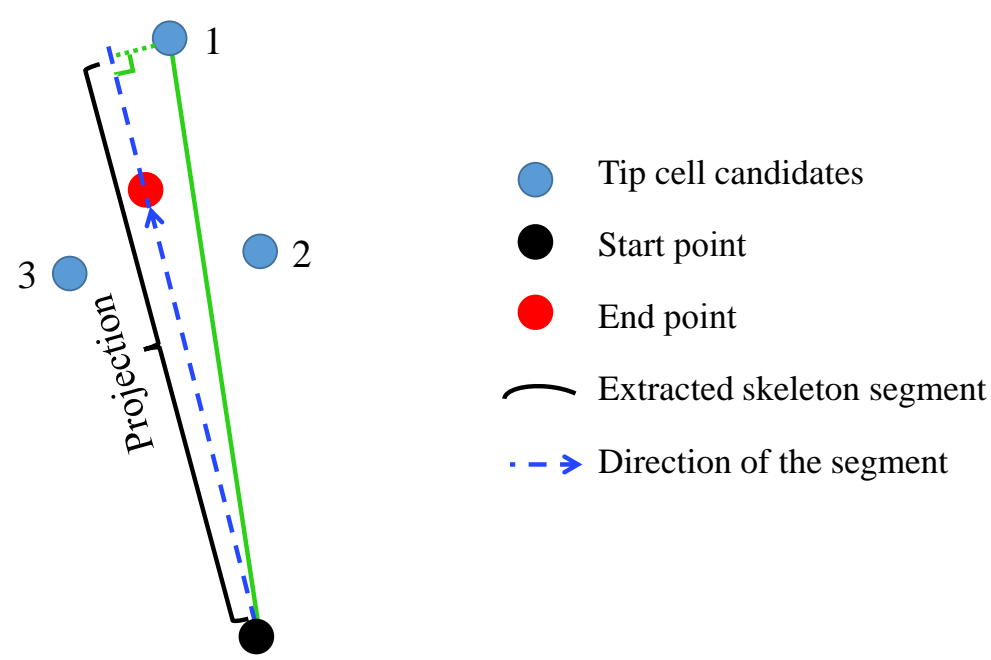

(b)

Figure 4.15: Diagram of tip cell identification: (a) tip cell candidates and (b) tip cell selection. 


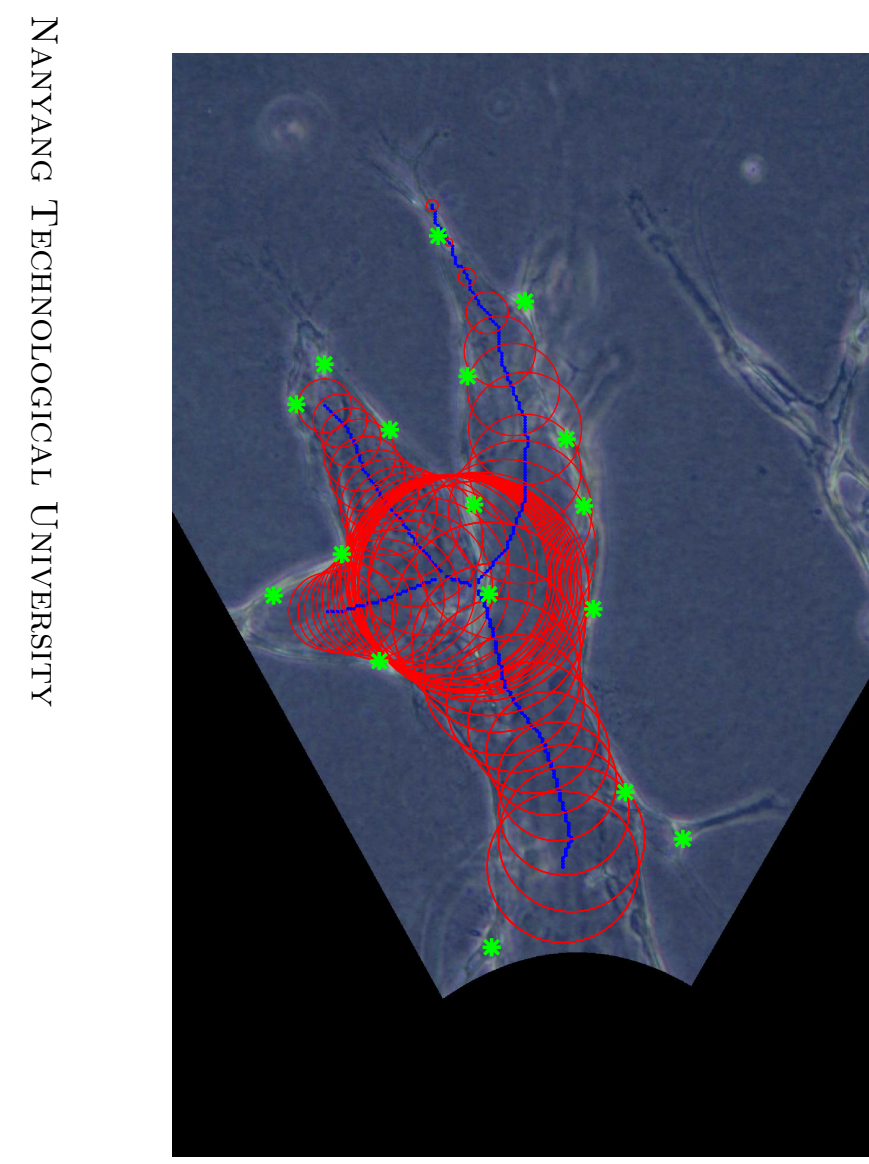

(a)

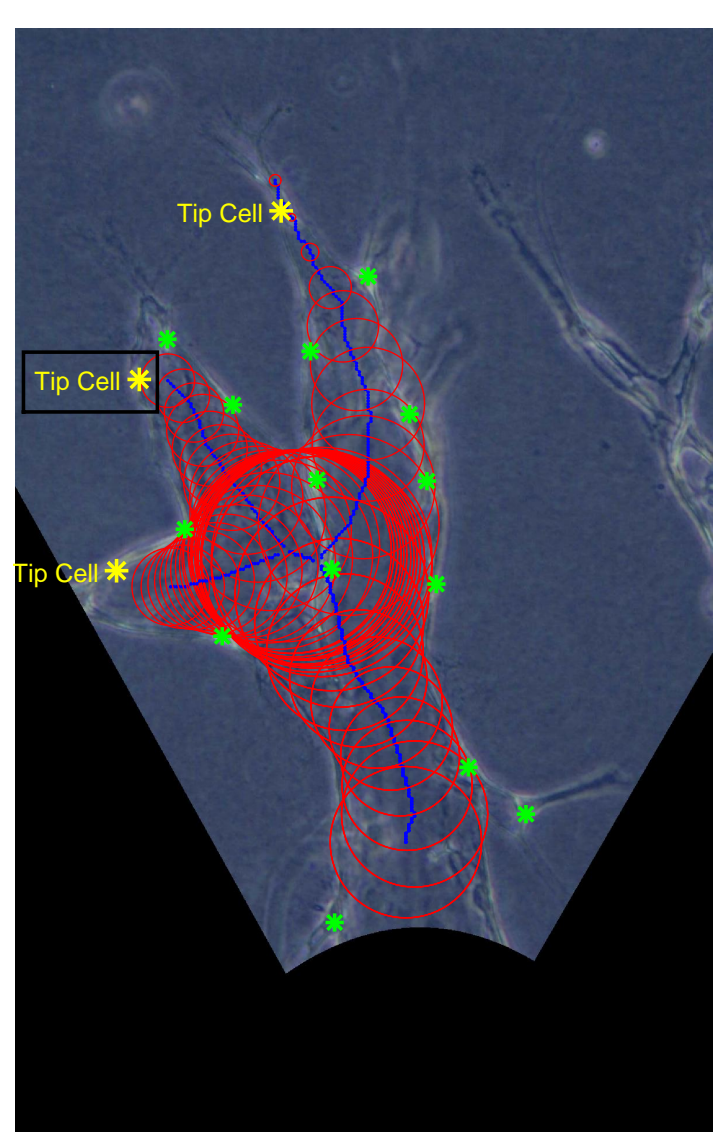

(b)

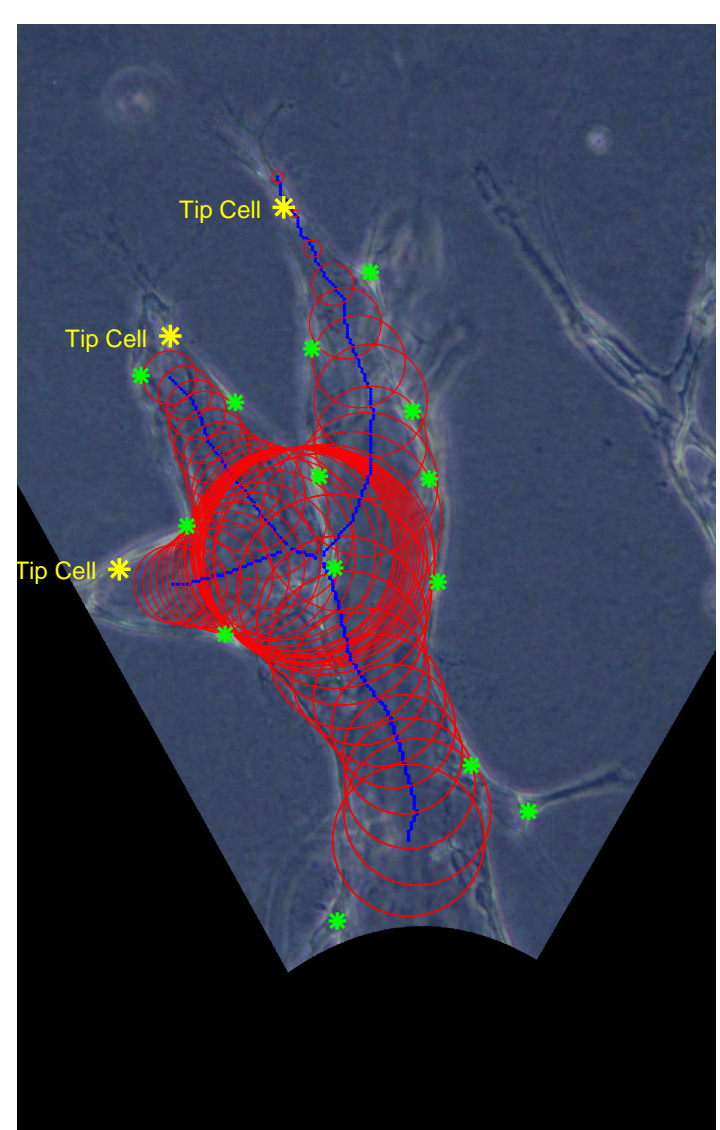

(c)

Figure 4.16: An example of tip cell identification result. (a) Angiogenic vessels with both cell and branch information: cell locations plotted as green stars, branch skeletons plotted as blue lines, and branch radii plotted as red circles; (b) Tip cells identified based on the distances between the cells and the end nodes only: tip cells plotted in yellow stars; and (c) Tip cells identified with our approach: tip cells plotted in yellow stars. 
with $\mathbf{x}_{e}, \mathbf{x}_{s}$ and $\mathbf{x}_{i}$ as the coordinates for the end point, start point, and the $i$ th tip cell candidate respectively. The candidate with largest projection value will be selected as the tip cell.

The cell closest to the end point can be either a tip cell or stalk cell. Therefore, selecting the cell nearest to the end point as the tip cell is not always a suitable choice. For instance, the cells depicted in Fig. 4.16(b) by yellow stars are the tip cells identified based on the distances only. It is noteworthy that the cell within the black rectangular box is wrongly identified. By incorporating the projection estimated by equation (4.35), this kind of errors can be avoided. The correctly identified tip cells are shown in Fig. 4.16(c).

We applied the method based on only distances and the proposed method on 62 branches to identify the tip cells. By comparing with the ground truths which obtained by visual inspection, the accuracy increases from $76.7 \%$ (the method based on only distances) to $90.3 \%$ (our proposed method).

\subsubsection{Investigation of S1P}

As explained in Section 1.1, the investigation of branching and anastomosis, which are key processes for the formation of a mature vascular, would lead to a better understanding of tip/stalk inter-transition. However, to our knowledge, the existing angiogenic studies in 3D in vitro experiments with HMVECs are unable to culture long vessels which are required for branching and anastomosis to occur.

$\mathrm{S} 1 \mathrm{P}$ is involved in angiogenesis to stimulate cell invasion, lumen formation, and branching morphogenesis. Nguyen et al. have shown that S1P increases the average vessel length by increasing the migration of HUVECs [56]. Therefore, we employ $\mathrm{S} 1 \mathrm{P}$ in our angiogenic experiments to form long angiogenic vessels. We consider three conditions of S1P (see Table 2.1).

We observe the angiogenic vessels under different conditions by phase contrast mi- 


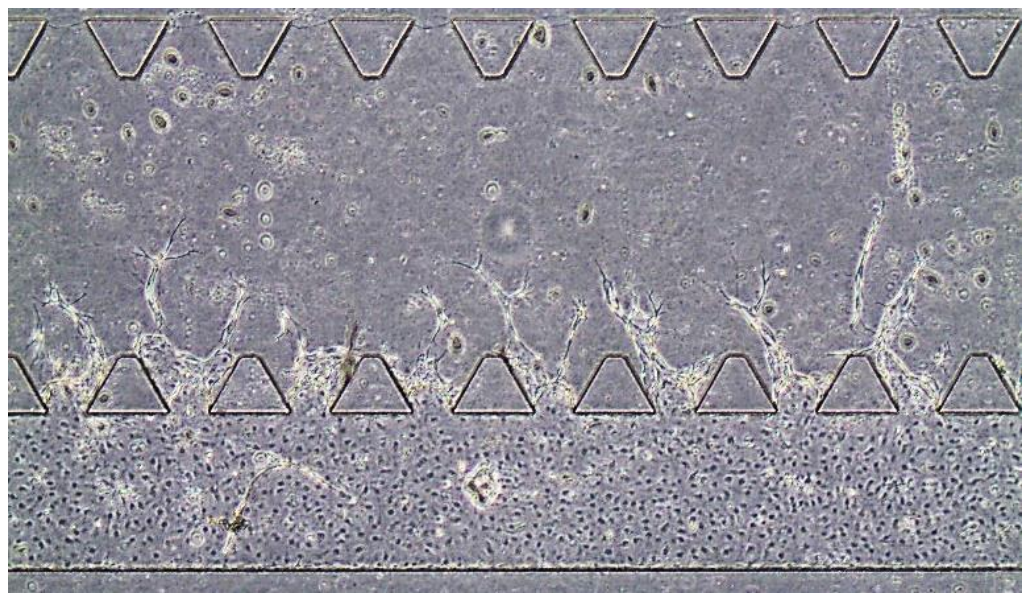

(a) No S1P.

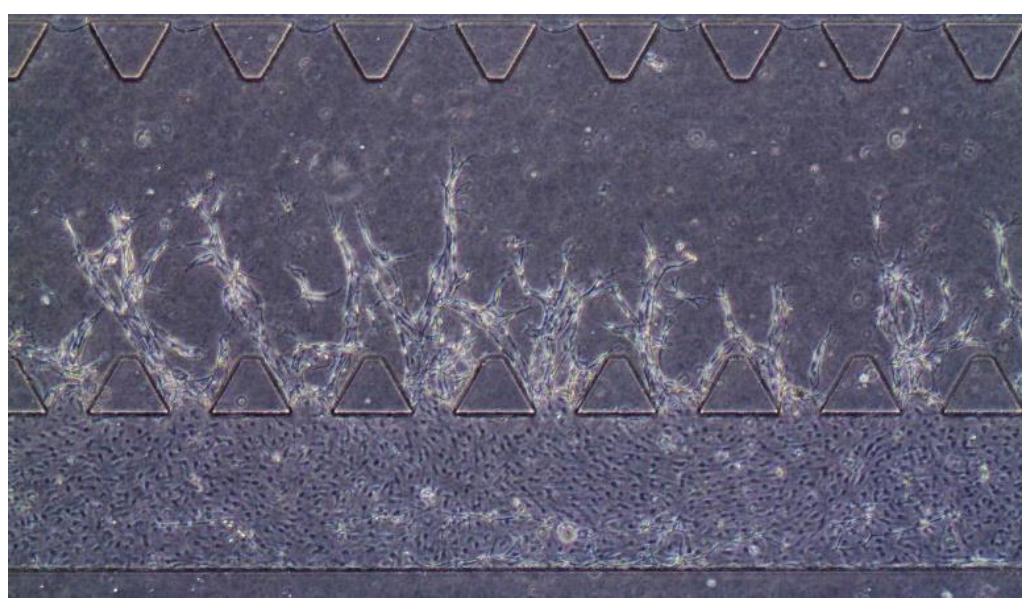

(b) Positive S1P gradient.

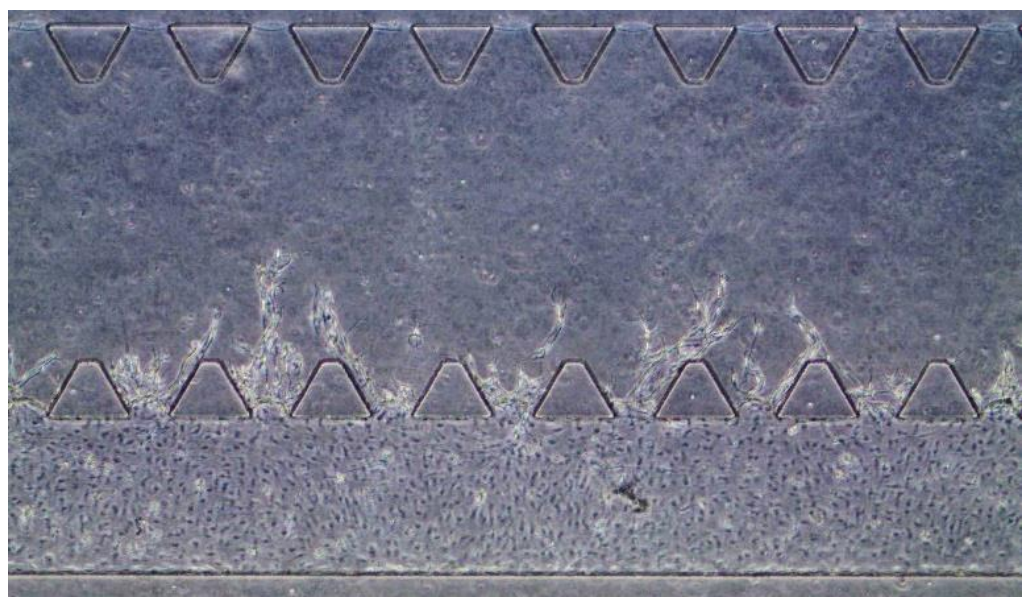

(c) Negative S1P gradient.

Figure 4.17: Angiogenic vessels observed by phase contrast microscopy (at 4x magnification) at day 9 under different S1P conditions: (a) No S1P, (b) Positive S1P gradient, and (c) Negative S1P gradient. 
croscopy at 4x and 20x magnification. The proposed AVFTS is applied to the phase contrast images at 20x magnification. To better illustrate the morphological difference of the angiogenic vessels under different S1P conditions, we provide their 4x phase contrast images (all acquired at day 9) in Fig. 4.17. From the examples in Fig. 4.17, we can observe that the vessels from positive S1P gradient (see Fig. 4.17(b)) are longer than in the other two conditions.

To quantitatively compare the vessel morphologies for the different S1P conditions, we apply the AVFTS proposed in Section 4.2 on the phase contrast images obtained at 20x magnification at day 9. Numerical parameters including the branch length, branch radius, and the number of branches in each sprouting region can be estimated.

We apply one-way analysis of variance (ANOVA) to examine the differences among the three S1P conditions on the branch length, branch radius, and the number of branches in a sprouting region. The $\mathrm{P}$ values for the ANOVA tests are shown in Fig. 4.18. The symbols in Fig. 4.18 are explained in Table 4.3.

Table 4.3: Symbols for different $\mathrm{P}$ values ranges.

\begin{tabular}{|c|c|c|}
\hline $\mathbf{P}$ value & Symbol & Meaning \\
\hline$P>0.05$ & ns & not significant \\
\hline $0.01<P \leq 0.05$ & $*$ & moderately significant \\
\hline $0.001<P \leq 0.01$ & $* *$ & significant \\
\hline $0.0001<P \leq 0.001$ & $* * *$ & very significant \\
\hline$P \leq 0.0001$ & $* * * *$ & extremely significant \\
\hline
\end{tabular}

For each plot in Fig. 4.18, $N$ is the sample size of each experimental condition. We analyzed 12 sprouting regions for each condition. Since Fig. 4.18(c) compares the number of branches in each sprouting region, $N=12$ for Fig. 4.18(c). Fig. 4.18(a) and 4.18(b) compare the average length and width of the branches. Due to the number of branches are different in these three experimental conditions, the $N$ values for each condition in Fig. 4.18(a) and 4.18(b) are different: $N=36$ for positive $\mathrm{S} 1 \mathrm{P}$ gradient, $N=24$ for negative $\mathrm{S} 1 \mathrm{P}$ gradient, and $N=25$ for no $\mathrm{S} 1 \mathrm{P}$. 


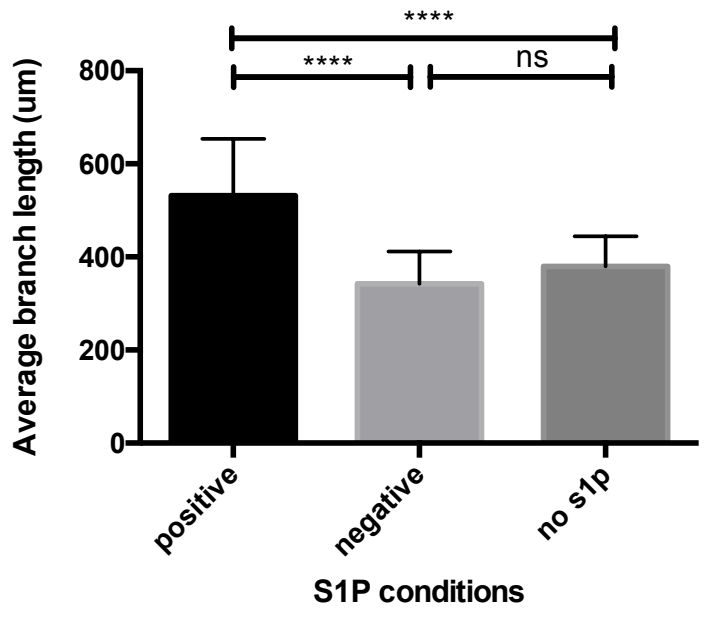

(a)

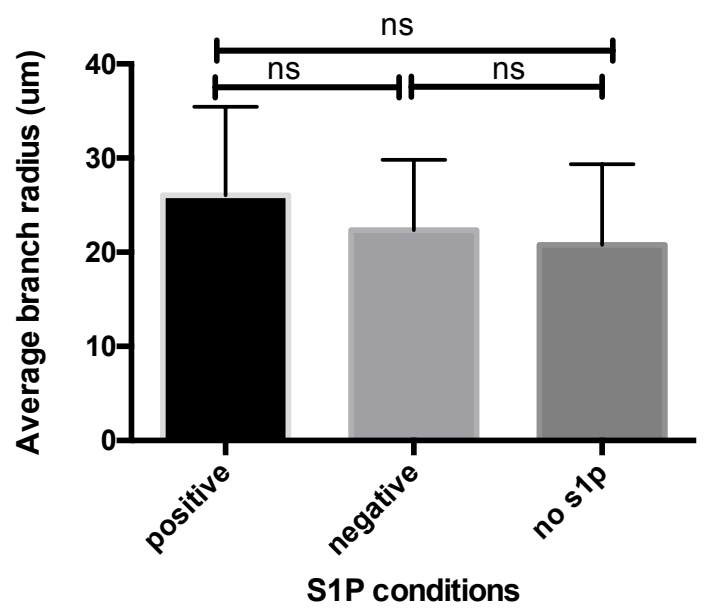

(b)

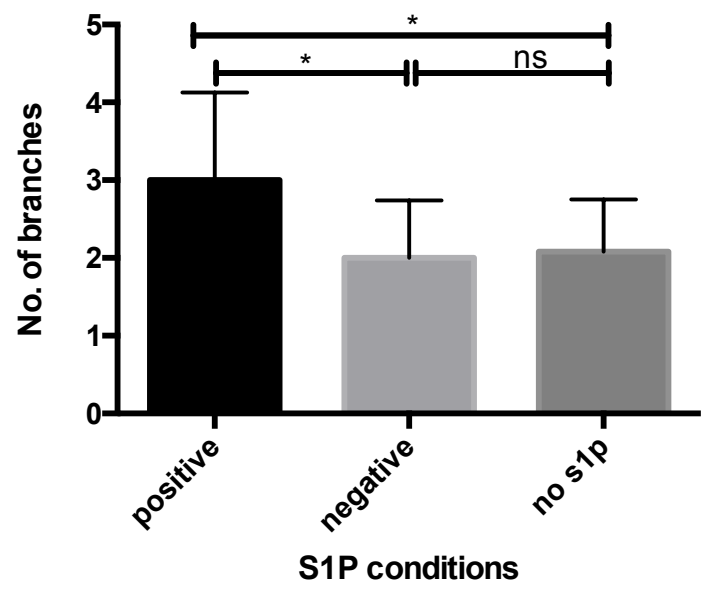

(c)

Figure 4.18: Quantitative comparison of vessel morphologies under different S1P conditions: (a) branch length $(N=24 \sim 36)$; (b) branch radius $(N=24 \sim 36)$; and (c) the number of branches in a sprouting region $(N=12)$. The error bars give the mean and standard deviation. 
Fig. 4.18(a) compares the average branch length for the different S1P conditions, showing that a positive S1P gradient induces significantly longer branches than the other two conditions. This observation implies that S1P in the medium channel regulates angiogenic sprouting by increasing ECs migration and vessel elongation, whereas S1P has no such effect in the cell channel.

The comparison of the average branch radius for the different S1P conditions is shown in Fig. 4.18(b). It indicates that S1P does not lead to significant difference among the three conditions.

As illustrated in Fig. 4.18(c), the average number of branches in a sprouting region under the a positive S1P gradient is larger than the other two conditions. Branching happens when the sprout extends over some distance, and there is sufficient space locally for a new branch to form [21]. We hypothesize that a positive S1P gradient increases the vessel length, leading to higher possibility for branching to occur.

Fig. 4.19 shows how the average branch length changes from day 4 to day 9 for different S1P conditions. We also plot the standard error of the mean (SEM) bars [146] to compare these three S1P conditions. The large gaps between bars between the positive S1P gradients and the other two conditions suggest that positive S1P gradients induce longer angiogenic vessels consistently over the 9-day period. More specifically, at day 9 , the vessels keep extending in the angiogenic experiments with positive S1P gradients, whereas they almost stop growing for the other two conditions.

In conclusion, positive S1P gradients promote angiogenic vessel growth and lead to more branches in a sprouting region. Branching and reconnection can be observed by providing positive S1P gradients in the angiogenic experiments in 3D MFDs, allowing biologists to gain a better understanding of angiogenesis. Moreover, when investigating the influences of different angiogenic factors on the angiogenic vessel morphologies, instead of estimating the morphological parameters manually, biologists can apply the proposed AVFTS to extract the morphological parameters to 


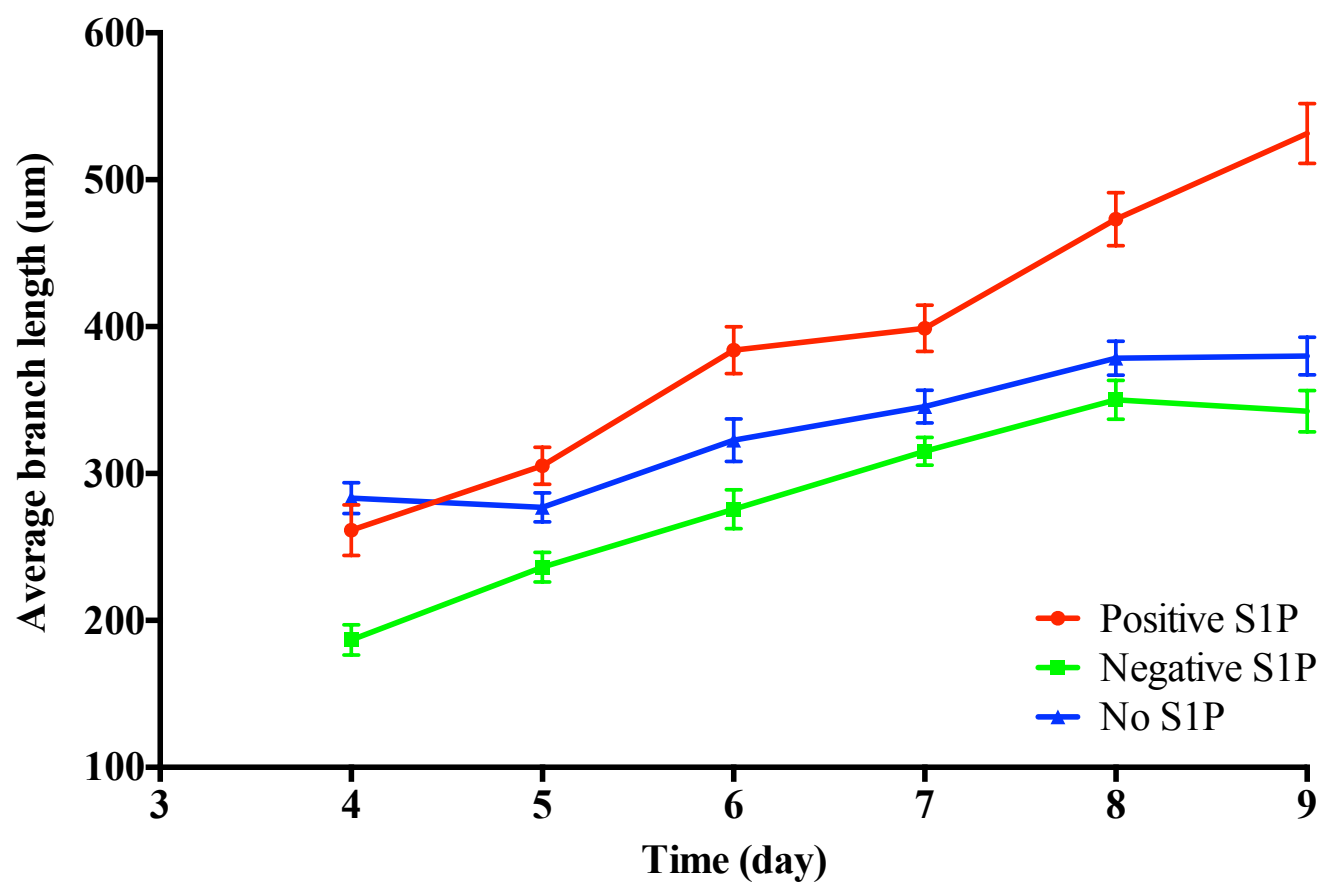

Figure 4.19: Change of average branch length over time for different S1P conditions: the error bars give the mean with SEM.

save time.

\subsection{Conclusion}

In this chapter, we have developed the AVFTS to automatically track the vessel formation, to extract quantitative vessel information, and to identify cell phenotypes from the experimental time-lapse phase contrast images. This system consists of preprocessing, skeletonization, and branch tracking. We first preprocess the experimental images to obtain the binary shapes. Next, we skeletonize the binary vessel shapes into segments by DT and AFMM. We then distinguish the specious segments that are not part of vessels by a binary SVM classifier. Lastly, we associate the branches over time through the Hungarian method. We are able to track the branch formation and extract branch information such as branch length, width, and number of branches in each sprouting region. 
Based on the cell locations from AMCTS and branch information from AVFTS, we identify the tip/stalk cell phenotypes. Specifically, in order to distinguish tip and stalk cells in a branch, we consider both the distances of the cells to the end point of its last segment and the projection estimated by equation (4.35). This would be useful for studying tip/stalk cell inter-transition so as to branching and reconnection process in angiogenesis.

Moreover, by automatically extracting quantitative vessel information such as vessel length, vessel width, and number of branches from phase contrast images, AVFTS benefits biologists who used to manually estimate the morphological parameters to compare the influence of different angiogenic factors. Specifically, for the angiogenic experiments with different S1P conditions, we extracted and compared the morphological changes in the vessels over time. We found that positive S1P gradient leads to not only more branches but also longer average branch length. 


\section{Chapter 5}

\section{Conclusions and Future Work}

In this thesis, we developed automated image analysis systems for the quantitative investigation of in vitro angiogenesis in 3D MFDs. This chapter concludes this thesis by summarizing the main contributions and suggesting directions for future research.

\subsection{Summary of Contributions}

The main contributions of this thesis are as follows:

- Experimental data of cultured HMVECs in 3D MFDs that grow angiogenic vessels at different S1P conditions over a period of 10 to 14 days

Branching and anastomosis are key processes to form mature vascular networks. As explained in Chapter 2, by adding S1P and VEGF in the angiogenic experiments, we cultured long vessels with branching and anastomosis using HMVECs in 3D MFDs. The angiogenic sprouting process is observed by a phase contrast microscope daily over 10 to 14 days and by a confocal micro- 
scope at the end time point. We developed automated image analysis systems and apply them to our experimental data with the purpose of quantitatively analyzing the behaviors of cell migration and vessel formation. To investigate the influence of S1P on angiogenesis vessel morphologies, we considered three different S1P conditions, as listed in Table 2.1. The experimental observations suggest that the vessels from the experiments under positive S1P gradient are longer than in the other two conditions.

\section{- Development of the AMCTS to automatically track the migration} of ECs within the angiogenic vessels

In Chapter 3, we developed the AMCTS to track the migrating ECs within the angiogenic vessels from the time-lapse phase contrast images. This system consists of three steps including preprocessing, cell detection, and multiple hypothesis Kalman filtering. In the preprocessing step, we construct the full view of long vessels by stitching the acquired images, align all the time-lapse images in a same sprouting region by image registration, and obtain the binary vessel shapes by vessel segmentation. Next, we detect and label the EC candidates from the phase contrast image sequences through template matching, where PLSR is applied for both offline training and classification. Lastly, we combine backward Kalman filtering with MHT to associate and track the extracted EC candidates over time. Since we captured the end-point confocal images with accurate cell locations, we initialize our tracks with the final time point after all proliferation has occurred, whereas other cell tracking applications start with the image at the first time point. Furthermore, we incorporate biological knowledge for estimating the track probability during cell association. Numerical results indicate that the proposed AMCTS is able to track the cell migration trajectories accurately. Cell lineage plots, showing the history of the cell proliferation and cell migration, are also generated automatically by this system. Such cell lineage plot provides crucial information (e.g., the number of cells, division time, and growth fraction) to biologists who are in- 
terested in studying cell migration behaviors in angiogenesis under different conditions.

- Development of the AVFTS to quantitatively analyze angiogenic vessel formation and to identify cell phenotypes

In Chapter 4, we proposed the AVFTS to automatically track the angiogenic vessel formation, and to extract quantitative vessel information from the time-lapse phase contrast images. The system consists of preprocessing, skeletonization, and branch tracking. In the preprocessing step, we obtain the binary vessel shapes. The preprocessing is identical as in the AMCTS. Next, we extract the connected skeleton segments with branch and end nodes by DT and AFMM. The specious segments, caused by noise or filopodia, are detected by a binary SVM classifier and removed. After identifying the route for each of the main branches, we apply the Hungarian method to associate the branches over time, yielding the branch trajectories. Branch information, including the branch length/width and the number of branches for each sprouting region, can be extracted by this system. Moreover, by combining the branch information with the cell locations obtained by AMCTS, we identified the cell phenotypes (tip or stalk cell). To study the influence of different angiogenic factors on angiogenic vessel morphologies, biologists can apply this system to extract quantitative information about the vessel morphologies from experimental microscopy images. To demonstrate the methodology, we conducted quantitative comparisons of the angiogenic vessels formed under different S1P conditions. We observed that positive S1P gradients promote angiogenic vessel growth and lead to more branches in a sprouting region. 


\subsection{Recommendations for Future Work}

\subsubsection{Multi-cell Tracking by Interacting Multiple Model Mo- tion Filter}

As discussed in Chapter 4, by applying AVFTS, we extracted branch information and identified the cell phenotypes. The interacting multiple model (IMM) algorithm can be applied to track multi-cell migration together with phenotypic changes $[147$, 148]. Specifically, instead of using one Kalman filter model for both cell phenotypes, the IMM algorithm incorporates two Kalman filter models, for instance, a first model for tip cells and a second model for stalk cells. These two Kalman filter models have different prediction equations and share the same update equations. The intertransition of cell phenotypes can be reflected as the transition between these two models. Fig. 5.1 illustrates the switching process of the two models in the IMM algorithm, which is assumed to be regulated by a finite-state Markov chain with a transition probability matrix (TPM):

$$
\mathrm{TPM}=\left[\begin{array}{ll}
p_{11} & p_{12} \\
p_{21} & p_{22}
\end{array}\right]
$$

where $p_{i j}$ is the probability of switching from model $i$ to model $j$ in consecutive frames for $i=1,2$ and $j=1,2$.

Table 5.1 explains the elements in TPM. $p_{12}$ is the probability of a tip cell becoming stalk cell. For a tip cell, $p_{12}$ is influenced by its distance to another tip or stalk cell in its neighboring sprouts and its migration velocity. For instance, if a tip cell locates

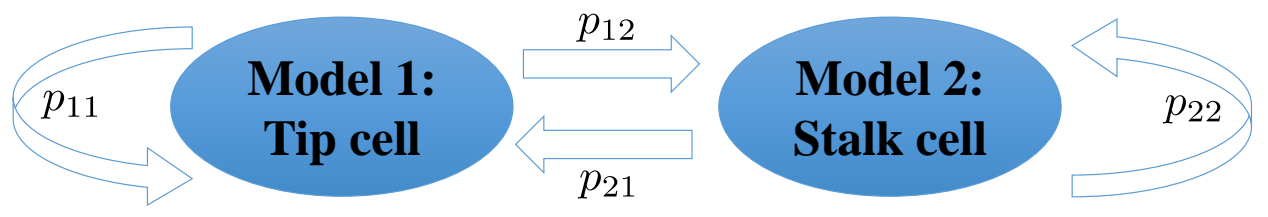

Figure 5.1: Interacting multiple model switching process. 
Table 5.1: Explanation of elements in TPM.

\begin{tabular}{|l|l|}
\hline Element & Meaning \\
\hline$p_{12}$ & tip cell becomes stalk cell (anastomosis) \\
\hline$p_{21}$ & stalk cell becomes tip cell (branching) \\
\hline$p_{11}$ & tip cell continues as tip cell \\
\hline$p_{22}$ & stalk cell continues as stalk cell \\
\hline
\end{tabular}

near to a neighboring sprouts and migrates towards this sprouts, anastomosis is likely to occur, hence $p_{12}$ would have a large value. Since anastomosis occurs at a well-defined distance [22, 149], large distance from the tip cell to the monolayer would lead to large value of $p_{12}$.

$p_{21}$ is the probability of stalk cell becoming tip cell. Since branching into a space occupied by another sprout is not possible, the value of $p_{21}$ would be small if there is no sufficient space locally for a new branch to form. Tip cells inhibit their neighboring cells from becoming tip cells. Thus, $p_{21}$ is also influenced by the number of cells between this stalk cell and its nearest tip cell. For instance, $p_{21}$ is small if the stalk cell is near to a tip cell.

After estimating the values for $p_{12}$ and $p_{21}, p_{11}$ and $p_{22}$ can be computed by:

$$
\begin{aligned}
& p_{11}=1-p_{12}, \\
& p_{22}=1-p_{21} .
\end{aligned}
$$

To track the multi-cell migration and the phenotypic changes over time, the equations to estimate the TPM and the prediction equations for both Kalman filter models need to be derived based on experimental data and data analysis as in Chapter 3 and Chapter 4. 


\subsubsection{Computation Models for Branching and Anastomosis in Angiogenesis}

Fig. 5.2 depicts the multiple aspects of investigating a biological process. Experiments are conducted to generate experimental data such as microscopy images. By quantitatively analyzing the experimental data through image analysis, numerical results can be used to build computational models. In turn, the computational models would help to better understand the biological process and improve the design of the experiments. Specifically, in this thesis we concentrate on angiogenesis.

In vitro angiogenic experiments are crucial for investigating and identifying the mechanisms of angiogenesis. We conducted angiogenic experiments in 3D MFDs in order to study and observe the collective cell behaviors and vessel formation under different experimental conditions.

Automated image processing tools are required to quantitatively analyze experimental images of angiogenesis. We developed image analysis systems to track the multi-cell migration and vascular formation over time, and to extract numerical

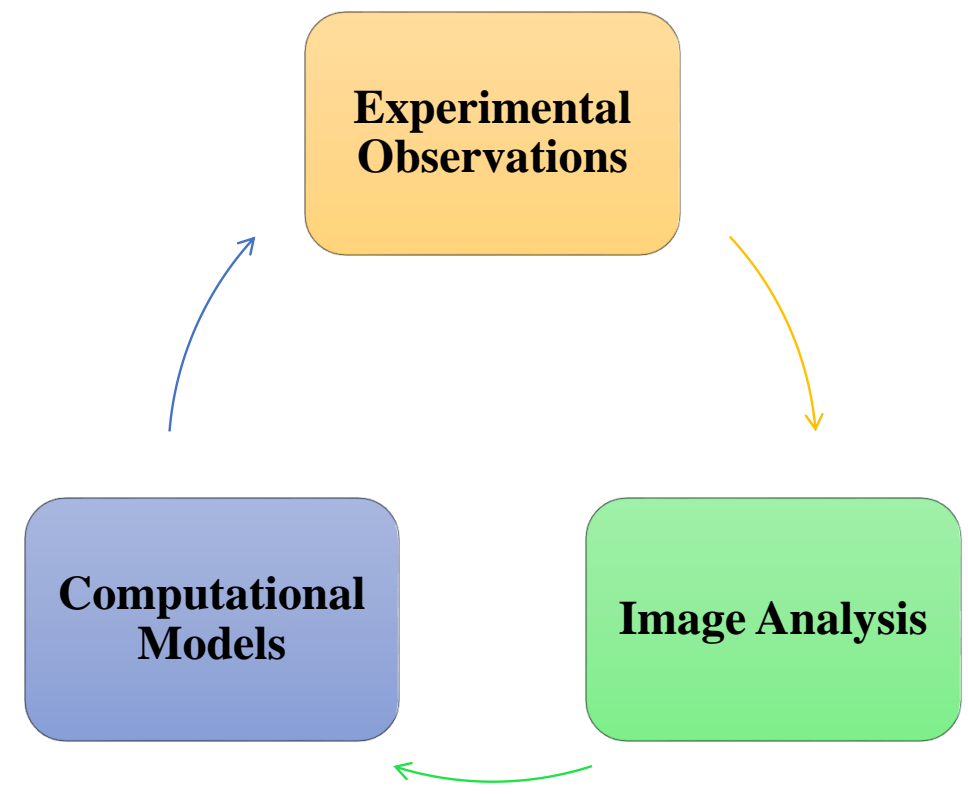

Figure 5.2: Multiple aspects of investigating a biological process. 
information of the cells and vessels such as cell locations, cell phenotypes, vessel skeletons, and vessel radii from the time-lapse phase contrast images. Biologists can apply these image analysis tools to quantitatively compare the cell migration behaviors and the angiogenic vessel morphologies for different angiogenic factors.

Mathematical models are commonly used to represent biological processes since they are accessible and convenient. They can be applied to identify the key factors of biological systems, to help optimize experimental design so as to save time and resources, to predict variables that are not experimentally accessible, and to generate accurate quantitative predictions in the biological systems. Existing computational models for angiogenesis rarely incorporate the occurrence of branching and anastomosis. To address this shortcoming, we may develop computational models from the numerical data (e.g. cell locations, cell phenotypes, and branch information) generated by the proposed systems. Such models might be able to predict the change of cell states (phenotypes) and cell coordinates (migration velocities). Branching and anastomosis may also be predicted, since these are processes often accompanied with cell phenotypic changes.

Such computational models are expected to help us gain a better understanding of the angiogenesis process, especially branching and anastomosis. 


\section{Author's Publications}

\section{Journal Papers:}

- M. Wang, L.L.S. Ong, J. Dauwels, and H.H. Asada, "Tracking of Endothelial Cell Networks from Coarse Time-lapse Phase Contrast Image," submitted to IEEE Transactions on Biomedical Engineering.

- M. Wang, L.L.S. Ong, J. Dauwels, and H.H. Asada, "Automated Tracking of Angiogenic Vessel Formation in 3D Microfluidic Device," submitted to PLOS ONE (under review).

\section{Conference Papers:}

- M. Wang, L.L.S. Ong, J. Dauwels, and H.H. Asada, "2D Data-driven Stalk Cell Prediction Model Based on Tip-stalk Cell Interaction in Angiogenesis," in 2013 35th Annual International Conference of the IEEE Engineering in Medicine and Biology Society (EMBC). IEEE, 2013, pp. 4537-4540.

- L.L.S. Ong, M. Wang, J. Dauwels, and H.H. Asada, "Segmentation of Densely Populated Cell Nuclei from Confocal Image Stacks using 3D Non-parametric Shape Priors," in 2014 36th Annual International Conference of the IEEE Engineering in Medicine and Biology Society (EMBC). IEEE, 2014, pp. 55265529 . 
- M. Wang, L.L.S. Ong, J. Dauwels, and H.H. Asada, "Automatic Detection of Endothelial Cells in 3D Angiogenic Sprouts from Experimental Phase Contrast Images," in SPIE Medical Imaging, pp. 94132I-94132I. International Society for Optics and Photonics, 2015.

- M. Wang, L.L.S. Ong, J. Dauwels, and H.H. Asada, "Automated Tracking of Cells from Phase Contrast Images by Multiple Hypothesis Kalman Filters", in Proc. IEEE Int. Conf. Acoust. Speech Signal Process. (ICASSP), pp. 942-946, 2015. 


\section{Bibliography}

[1] B. R. Zetter, PhD, "Angiogenesis and tumor metastasis," Annual review of medicine, vol. 49, no. 1, pp. 407-424, 1998.

[2] W. Farahat, L. Wood, I. Zervantonakis, A. Schor, S. Ong, D. Neal, R. Kamm, and H. Asada, "Ensemble analysis of angiogenic growth in three-dimensional microfluidic cell cultures," PloS one, vol. 7, no. 5, p. e37333, 2012.

[3] T. Fawcett, "An introduction to roc analysis," Pattern recognition letters, vol. 27 , no. 8 , pp. $861-874,2006$.

[4] H. Gerhardt, "Vegf and endothelial guidance in angiogenic sprouting," VEGF in Development, pp. 68-78, 2008.

[5] J. Folkman and C. Haudenschild, "Angiogenesis in vitro," 1980.

[6] N. Mantzaris, S. Webb, and H. Othmer, "Mathematical modeling of tumorinduced angiogenesis," Journal of mathematical biology, vol. 49, no. 2, pp. 111-187, 2004.

[7] C. Fitzmaurice, D. Dicker, A. Pain, H. Hamavid, M. Moradi-Lakeh, M. F. MacIntyre, C. Allen, G. Hansen, R. Woodbrook, C. Wolfe et al., "The global burden of cancer 2013," JAMA oncology, vol. 1, no. 4, pp. 505-527, 2015.

[8] A. C. Society, "Cancer facts and figures 2016," 2016.

[9] R. L. Siegel, K. D. Miller, and A. Jemal, "Cancer statistics, 2016," CA: a cancer journal for clinicians, vol. 66, no. 1, pp. 7-30, 2016. 
[10] R. Lozano, M. Naghavi, K. Foreman, S. Lim, K. Shibuya, V. Aboyans, J. Abraham, T. Adair, R. Aggarwal, S. Y. Ahn et al., "Global and regional mortality from 235 causes of death for 20 age groups in 1990 and 2010: a systematic analysis for the global burden of disease study 2010," The Lancet, vol. 380, no. 9859, pp. 2095-2128, 2013.

[11] J. Ferlay, I. Soerjomataram, R. Dikshit, S. Eser, C. Mathers, M. Rebelo, D. M. Parkin, D. Forman, and F. Bray, "Cancer incidence and mortality worldwide: sources, methods and major patterns in globocan 2012," International journal of cancer, vol. 136, no. 5, pp. E359-E386, 2015.

[12] F. Nussenbaum and I. M. Herman, "Tumor angiogenesis: insights and innovations," Journal of oncology, vol. 2010, 2010.

[13] P. Carmeliet and R. K. Jain, "Angiogenesis in cancer and other diseases," nature, vol. 407, no. 6801, pp. 249-257, 2000.

[14] D. Liao and R. S. Johnson, "Hypoxia: a key regulator of angiogenesis in cancer," Cancer and Metastasis Reviews, vol. 26, no. 2, pp. 281-290, 2007.

[15] R. Blanco and H. Gerhardt, "Vegf and notch in tip and stalk cell selection," Cold Spring Harbor perspectives in medicine, vol. 3, no. 1, p. a006569, 2013.

[16] S. M. Weis and D. A. Cheresh, "Tumor angiogenesis: molecular pathways and therapeutic targets," Nature medicine, vol. 17, no. 11, pp. 1359-1370, 2011.

[17] Z. Wang, C. Dabrosin, X. Yin, M. M. Fuster, A. Arreola, W. K. Rathmell, D. Generali, G. P. Nagaraju, B. El-Rayes, D. Ribatti et al., "Broad targeting of angiogenesis for cancer prevention and therapy," in Seminars in cancer biology, vol. 35. Elsevier, 2015, pp. S224-S243.

[18] F. De Smet, I. Segura, K. De Bock, P. Hohensinner, and P. Carmeliet, "Mechanisms of vessel branching filopodia on endothelial tip cells lead the way," Arteriosclerosis, thrombosis, and vascular biology, vol. 29, no. 5, pp. 639-649, 2009. 
[19] L. Jakobsson, C. A. Franco, K. Bentley, R. T. Collins, B. Ponsioen, I. M. Aspalter, I. Rosewell, M. Busse, G. Thurston, A. Medvinsky et al., "Endothelial cells dynamically compete for the tip cell position during angiogenic sprouting," Nature cell biology, vol. 12, no. 10, pp. 943-953, 2010.

[20] A. F. Siekmann, M. Affolter, and H.-G. Belting, "The tip cell concept ten years after: new players tune in for a common theme," Experimental cell research, vol. 319, pp. 1255-1263, 2013.

[21] H. M. Eilken and R. H. Adams, "Dynamics of endothelial cell behavior in sprouting angiogenesis," Current opinion in cell biology, vol. 22, no. 5, pp. 617-625, 2010.

[22] N. Paweletz and M. Knierim, "Tumor-related angiogenesis," Critical reviews in oncology/hematology, vol. 9, no. 3, pp. 197-242, 1989.

[23] F. Milde, M. Bergdorf, and P. Koumoutsakos, "A hybrid model for threedimensional simulations of sprouting angiogenesis," Biophysical journal, vol. 95, no. 7, pp. 3146-3160, 2008.

[24] S. Davis, T. H. Aldrich, P. F. Jones, A. Acheson, D. L. Compton, V. Jain, T. E. Ryan, J. Bruno, C. Radziejewski, P. C. Maisonpierre et al., "Isolation of angiopoietin-1, a ligand for the tie2 receptor, by secretion-trap expression cloning," Cell, vol. 87, no. 7, pp. 1161-1169, 1996.

[25] A. Slungaard, "Platelet factor 4: a chemokine enigma," The international journal of biochemistry \& cell biology, vol. 37, no. 6, pp. 1162-1167, 2005.

[26] M. Klagsbrun and M. A. Moses, "Molecular angiogenesis," Chemistry \& biology, vol. 6, no. 8, pp. R217-R224, 1999.

[27] N. Ferrara, H.-P. Gerber, and J. LeCouter, "The biology of vegf and its receptors," Nature medicine, vol. 9, no. 6, pp. 669-676, 2003. 
[28] D. T. Connolly, D. M. Heuvelman, R. Nelson, J. V. Olander, B. L. Eppley, J. Delfino, N. Siegel, R. Leimgruber, and J. Feder, "Tumor vascular permeability factor stimulates endothelial cell growth and angiogenesis." Journal of Clinical Investigation, vol. 84, no. 5, p. 1470, 1989.

[29] A. Eichmann, C. Corbel, V. Nataf, P. Vaigot, C. Bréant, and N. M. Le Douarin, "Ligand-dependent development of the endothelial and hemopoietic lineages from embryonic mesodermal cells expressing vascular endothelial growth factor receptor 2," Proceedings of the National Academy of Sciences, vol. 94, no. 10, pp. 5141-5146, 1997.

[30] G. Conn, D. D. Soderman, M.-T. Schaeffer, M. Wile, V. B. Hatcher, and K. A. Thomas, "Purification of a glycoprotein vascular endothelial cell mitogen from a rat glioma-derived cell line." Proceedings of the National Academy of Sciences, vol. 87, no. 4, pp. 1323-1327, 1990.

[31] S. Dimmeler, E. Dernbach, and A. M. Zeiher, "Phosphorylation of the endothelial nitric oxide synthase at ser-1177 is required for vegf-induced endothelial cell migration," FEBS letters, vol. 477, no. 3, pp. 258-262, 2000.

[32] H.-P. Gerber, A. McMurtrey, J. Kowalski, M. Yan, B. A. Keyt, V. Dixit, and N. Ferrara, "Vascular endothelial growth factor regulates endothelial cell survival through the phosphatidylinositol 3-kinase/akt signal transduction pathway requirement for flk-1/kdr activation," Journal of Biological Chemistry, vol. 273, no. 46, pp. $30336-30343,1998$.

[33] S. Spiegel, D. English, and S. Milstien, "Sphingosine 1-phosphate signaling: providing cells with a sense of direction," Trends in cell biology, vol. 12, no. 5, pp. 236-242, 2002.

[34] R. Nema, "emerging role of sphingosine-1-phosphate signaling in head and neck squamous cell carcinoma," OncoTargets and Therapy, vol. 9, pp. 3269$3280,2016$. 
[35] S. K. Alford, Endothelial Cell Migration in the Presence of Multiple Stimuli: Measurement and Systems Analysis of Sphingosine 1-phosphate Stimulated Cell Migration. ProQuest, 2008.

[36] G. V. Sherbet, Growth factors and their receptors in cell differentiation, cancer and cancer therapy. Elsevier, 2011.

[37] Y. A. Hannun, C. Luberto, C. Mao, and L. M. Obeid, Bioactive Sphingolipids in Cancer Biology and Therapy. Springer, 2015.

[38] B. Alberts, Molecular Biology of the Cell with CD. Garland, 2008.

[39] G. Michel, T. Tonon, D. Scornet, J. M. Cock, and B. Kloareg, "The cell wall polysaccharide metabolism of the brown alga ectocarpus siliculosus. insights into the evolution of extracellular matrix polysaccharides in eukaryotes," New Phytologist, vol. 188, no. 1, pp. 82-97, 2010.

[40] C. Frantz, K. M. Stewart, and V. M. Weaver, "The extracellular matrix at a glance," J Cell Sci, vol. 123, no. 24, pp. 4195-4200, 2010.

[41] G. M. Cooper, "Cell walls and the extracellular matrix," 2000.

[42] R. K. Assoian and E. A. Klein, "Growth control by intracellular tension and extracellular stiffness," Trends in cell biology, vol. 18, no. 7, pp. 347-352, 2008.

[43] R. B. Vernon and E. H. Sage, "Between molecules and morphology. extracellular matrix and creation of vascular form." The American journal of pathology, vol. 147, no. 4, p. 873, 1995.

[44] T. Gridley, "Chapter nine-notch signaling in the vasculature," Current topics in developmental biology, vol. 92, pp. 277-309, 2010.

[45] R. D. Travasso, E. C. Poiré, M. Castro, J. C. Rodrguez-Manzaneque, and A. Hernández-Machado, "Tumor angiogenesis and vascular patterning: a mathematical model," PloS one, vol. 6, no. 5, p. e19989, 2011. 
[46] Y. Yokota, H. Nakajima, Y. Wakayama, A. Muto, K. Kawakami, S. Fukuhara, and N. Mochizuki, "Endothelial ca2+ oscillations reflect vegfr signalingregulated angiogenic capacity in vivo," eLife, vol. 4, p. e08817, 2015.

[47] S. S. Hasan and A. F. Siekmann, "The same but different: signaling pathways in control of endothelial cell migration," Current opinion in cell biology, vol. 36, pp. 86-92, 2015.

[48] J. C. Chappell and V. L. Bautch, "Chapter two-vascular development: Genetic mechanisms and links to vascular disease," Current topics in developmental biology, vol. 90, pp. 43-72, 2010.

[49] J.-W. Gu, P. Rizzo, A. Pannuti, T. Golde, B. Osborne, and L. Miele, "Notch signals in the endothelium and cancer" stem-like" cells: opportunities for cancer therapy," Vascular cell, vol. 4, no. 1, p. 1, 2012.

[50] A. Carlier, L. Geris, K. Bentley, G. Carmeliet, P. Carmeliet, and H. Van Oosterwyck, "Mosaic: a multiscale model of osteogenesis and sprouting angiogenesis with lateral inhibition of endothelial cells," PLoS Comput Biol, vol. 8, no. 10, p. e1002724, 2012.

[51] C. Betz, A. Lenard, H.-G. Belting, and M. Affolter, "Cell behaviors and dynamics during angiogenesis," Development, vol. 143, no. 13, pp. 2249-2260, 2016.

[52] L. Wood, R. Kamm, and H. Asada, "Stochastic modeling and identification of emergent behaviors of an endothelial cell population in angiogenic pattern formation," The International Journal of Robotics Research, vol. 30, no. 6, pp. 659-677, 2011.

[53] S. K. Das, S. Chung, I. Zervantonakis, J. Atnafu, and R. D. Kamm, "A microfluidic platform for studying the effects of small temperature gradients in an incubator environment," Biomicrofluidics, vol. 2, no. 3, p. 034106, 2008. 
[54] L. Boisen, K. R. Drasbek, A. S. Pedersen, and P. Kristensen, "Evaluation of endothelial cell culture as a model system of vascular ageing," Experimental gerontology, vol. 45, no. 10, pp. 779-787, 2010.

[55] S. H. Lim, C. Kim, A. R. Aref, R. D. Kamm, and M. Raghunath, "Complementary effects of ciclopirox olamine, a prolyl hydroxylase inhibitor and sphingosine 1-phosphate on fibroblasts and endothelial cells in driving capillary sprouting," Integrative Biology, vol. 5, no. 12, pp. 1474-1484, 2013.

[56] D.-H. T. Nguyen, S. C. Stapleton, M. T. Yang, S. S. Cha, C. K. Choi, P. A. Galie, and C. S. Chen, "Biomimetic model to reconstitute angiogenic sprouting morphogenesis in vitro," Proceedings of the National Academy of Sciences, vol. 110, no. 17, pp. 6712-6717, 2013.

[57] R. Suman, G. Smith, K. E. Hazel, R. Kasprowicz, M. Coles, P. OToole, and S. Chawla, "Label-free imaging to study phenotypic behavioural traits of cells in complex co-cultures," Scientific reports, vol. 6, 2016.

[58] Y. Song, A. Bahnson, N. Hall, H. Yu, H. Shen, D. Koebler, R. Houck, Y. Xie, and T. Cheng, "Stem cell traits in long-term co-culture revealed by time-lapse imaging," Leukemia, vol. 24, no. 1, pp. 153-161, 2010.

[59] E. E. Zattara, K. W. Turlington, and A. E. Bely, "Long-term time-lapse live imaging reveals extensive cell migration during annelid regeneration," $B M C$ developmental biology, vol. 16, no. 1, p. 1, 2016.

[60] F. Progatzky, M. J. Dallman, and C. L. Celso, "From seeing to believing: labelling strategies for in vivo cell-tracking experiments," Interface Focus, vol. 3, no. 3, p. 20130001, 2013.

[61] D. B. Murphy, Fundamentals of light microscopy and electronic imaging. John Wiley \& Sons, 2002.

[62] L. Lamalice, F. Le Boeuf, and J. Huot, "Endothelial cell migration during angiogenesis," Circulation research, vol. 100, no. 6, pp. 782-794, 2007. 
[63] M. Schiegg, P. Hanslovsky, C. Haubold, U. Koethe, L. Hufnagel, and F. A. Hamprecht, "Graphical model for joint segmentation and tracking of multiple dividing cells," Bioinformatics, p. btu764, 2014.

[64] T. Kanade, Z. Yin, R. Bise, S. Huh, S. Eom, M. F. Sandbothe, and M. Chen, "Cell image analysis: Algorithms, system and applications," in Applications of Computer Vision (WACV), 2011 IEEE Workshop on. IEEE, 2011, pp. $374-381$.

[65] N. Wei, J. You, K. Friehs, E. Flaschel, and T. W. Nattkemper, "An in situ probe for on-line monitoring of cell density and viability on the basis of dark field microscopy in conjunction with image processing and supervised machine learning," Biotechnology and bioengineering, vol. 97, no. 6, pp. 1489-1500, 2007.

[66] L. L. S. Ong, J. Dauwels, M. H. Ang, and H. H. Asada, "A bayesian filtering approach to incorporate $2 \mathrm{~d} / 3 \mathrm{~d}$ time-lapse confocal images for tracking angiogenic sprouting cells interacting with the gel matrix," Medical image analysis, vol. 18, no. 1, pp. 211-227, 2014.

[67] L. Eklund, M. Bry, and K. Alitalo, "Mouse models for studying angiogenesis and lymphangiogenesis in cancer," Molecular oncology, vol. 7, no. 2, pp. 259$282,2013$.

[68] M. N. Chávez, G. Aedo, F. A. Fierro, M. L. Allende, and J. T. Egaña, "Zebrafish as an emerging model organism to study angiogenesis in development and regeneration," Frontiers in physiology, vol. 7, 2016.

[69] J. Stephens, "Lobund-wistar rat," https://visualsonline.cancer.gov/details. cfm?imageid=2568, June 1992, (Accessed on 07/11/2016).

[70] A. Chow, "Cell cycle control by oncogenes and tumor suppressors: driving the transformation of normal cells into cancerous cells," Nature Education, vol. 3, no. $7,2010$. 
[71] S. Chung, R. Sudo, P. J. Mack, C.-R. Wan, V. Vickerman, and R. D. Kamm, "Cell migration into scaffolds under co-culture conditions in a microfluidic platform," Lab on a Chip, vol. 9, no. 2, pp. 269-275, 2009.

[72] C. J. Jackson and M. Nguyen, "Human microvascular endothelial cells differ from macrovascular endothelial cells in their expression of matrix metalloproteinases," The international journal of biochemistry \& cell biology, vol. 29, no. 10, pp. 1167-1177, 1997.

[73] S. Li, N. F. Huang, and S. Hsu, "Mechanotransduction in endothelial cell migration," Journal of cellular biochemistry, vol. 96, no. 6, pp. 1110-1126, 2005.

[74] R. L. Klemke, S. Cai, A. L. Giannini, P. J. Gallagher, P. De Lanerolle, and D. A. Cheresh, "Regulation of cell motility by mitogen-activated protein kinase," The Journal of cell biology, vol. 137, no. 2, pp. 481-492, 1997.

[75] D. E. Ingber, "Integrins, tensegrity, and mechanotransduction," Gravitational and Space Research, vol. 10, no. 2, 2007.

[76] — _ "Tensegrity i. cell structure and hierarchical systems biology," Journal of cell science, vol. 116, no. 7, pp. 1157-1173, 2003.

[77] I. Tensegrity, "How structural networks influence cellular information processing networks, donald e. ingber," Journal of Cell Science, 2003.

[78] A. Dufour, V. Shinin, S. Tajbakhsh, N. Guillén-Aghion, J.-C. Olivo-Marin, and C. Zimmer, "Segmenting and tracking fluorescent cells in dynamic 3-d microscopy with coupled active surfaces," Image Processing, IEEE Transactions on, vol. 14, no. 9, pp. 1396-1410, 2005.

[79] D. Padfield, J. Rittscher, N. Thomas, and B. Roysam, "Spatio-temporal cell cycle phase analysis using level sets and fast marching methods," Medical image analysis, vol. 13, no. 1, pp. 143-155, 2009. 
[80] A. Sacan, H. Ferhatosmanoglu, and H. Coskun, "Celltrack: an open-source software for cell tracking and motility analysis," Bioinformatics, vol. 24, no. 14, pp. 1647-1649, 2008.

[81] F. Amat, W. Lemon, D. P. Mossing, K. McDole, Y. Wan, K. Branson, E. W. Myers, and P. J. Keller, "Fast, accurate reconstruction of cell lineages from large-scale fluorescence microscopy data," Nature methods, 2014.

[82] I. Ersoy, F. Bunyak, M. A. Mackey, and K. Palaniappan, "Cell segmentation using hessian-based detection and contour evolution with directional derivatives," in Image Processing, 2008. ICIP 2008. 15th IEEE International Conference on. IEEE, 2008, pp. 1804-1807.

[83] F. Ambriz-Colin, M. Torres-Cisneros, J. Avina-Cervantes, J. SaavedraMartinez, O. Debeir, and J. Sanchez-Mondragon, "Detection of biological cells in phase-contrast microscopy images," in Artificial Intelligence, 2006. MICAI'06. Fifth Mexican International Conference on. IEEE, 2006, pp. 6877.

[84] C.-H. Huang, S. Sankaran, D. Racoceanu, S. Hariharan, and S. Ahmed, "Online 3-d tracking of suspension living cells imaged with phase-contrast microscopy," Biomedical Engineering, IEEE Transactions on, vol. 59, no. 7, pp. 1924-1933, 2012.

[85] H. Su, Z. Yin, T. Kanade, and S. Huh, "Phase contrast image restoration via dictionary representation of diffraction patterns," in International Conference on Medical Image Computing and Computer-Assisted Intervention. Springer, 2012, pp. 615-622.

[86] Z. Yin, T. Kanade, and M. Chen, "Understanding the phase contrast optics to restore artifact-free microscopy images for segmentation," Medical image analysis, vol. 16, no. 5, pp. 1047-1062, 2012. 
[87] D. Young, C. Glasbey, A. Gray, and N. Martin, "Towards automatic cell identification in dic microscopy," Journal of Microscopy, vol. 192, no. 2, pp. 186-193, 1998.

[88] R. C. Gonzalez, Digital image processing. Pearson Education India, 2009.

[89] J. W. Han, T. P. Breckon, D. A. Randell, and G. Landini, "The application of support vector machine classification to detect cell nuclei for automated microscopy," Machine Vision and Applications, vol. 23, no. 1, pp. 15-24, 2012.

[90] M. Wang, X. Zhou, F. Li, J. Huckins, R. W. King, and S. T. Wong, "Novel cell segmentation and online svm for cell cycle phase identification in automated microscopy," Bioinformatics, vol. 24, no. 1, pp. 94-101, 2008.

[91] Z. Yin, R. Bise, M. Chen, and T. Kanade, "Cell segmentation in microscopy imagery using a bag of local bayesian classifiers," in Biomedical Imaging: From Nano to Macro, 2010 IEEE International Symposium on. IEEE, 2010, pp. $125-128$.

[92] X. Chen, X. Zhou, and S. T. Wong, "Automated segmentation, classification, and tracking of cancer cell nuclei in time-lapse microscopy," Biomedical Engineering, IEEE Transactions on, vol. 53, no. 4, pp. 762-766, 2006.

[93] Z. N. Demou and L. V. McIntire, "Fully automated three-dimensional tracking of cancer cells in collagen gels determination of motility phenotypes at the cellular level," Cancer Research, vol. 62, no. 18, pp. 5301-5307, 2002.

[94] I. Adanja, V. Megalizzi, O. Debeir, and C. Decaestecker, "A new method to address unmet needs for extracting individual cell migration features from a large number of cells embedded in 3d volumes," PloS one, vol. 6, no. 7, p. e22263, 2011.

[95] I. Adanja, O. Debeir, V. Mégalizzi, R. Kiss, N. Warzée, and C. Decaestecker, "Automated tracking of unmarked cells migrating in three-dimensional ma- 
trices applied to anti-cancer drug screening," Experimental cell research, vol. 316, no. 2, pp. 181-193, 2010.

[96] O. Debeir, P. Van Ham, R. Kiss, and C. Decaestecker, "Tracking of migrating cells under phase-contrast video microscopy with combined mean-shift processes," Medical Imaging, IEEE Transactions on, vol. 24, no. 6, pp. 697-711, 2005.

[97] H. Kuhn, "The hungarian method for the assignment problem," Naval Research Logistics (NRL), vol. 52, no. 1, pp. 7-21, 2005.

[98] K. E. Magnusson and J. Jaldén, "A batch algorithm using iterative application of the viterbi algorithm to track cells and construct cell lineages," in 2012 9th IEEE International Symposium on Biomedical Imaging (ISBI). IEEE, 2012, pp. 382-385.

[99] X. Yang, H. Li, and X. Zhou, "Nuclei segmentation using marker-controlled watershed, tracking using mean-shift, and kalman filter in time-lapse microscopy," Circuits and Systems I: Regular Papers, IEEE Transactions on, vol. 53, no. 11, pp. 2405-2414, 2006.

[100] I. Smal, W. Niessen, and E. Meijering, "Particle filtering for multiple object tracking in molecular cell biology," in Nonlinear Statistical Signal Processing Workshop, 2006 IEEE. IEEE, 2006, pp. 129-132.

[101] F. Bunyak, K. Palaniappan, S. K. Nath, T. Baskin, and G. Dong, "Quantitative cell motility for in vitro wound healing using level set-based active contour tracking," in Biomedical Imaging: Nano to Macro, 2006. 3rd IEEE International Symposium on. IEEE, 2006, pp. 1040-1043.

[102] L.-L. Ong, V. Ang, and H. H. Asada, "Tracking of cell population from time lapse and end point confocal microscopy images with multiple hypothesis kalman smoothing filters," in Computer Vision and Pattern Recognition 
Workshops (CVPRW), 2010 IEEE Computer Society Conference on. IEEE, 2010, pp. $71-78$.

[103] X. Lou, M. Schiegg, and F. A. Hamprecht, "Active structured learning for cell tracking: algorithm, framework, and usability," IEEE transactions on medical imaging, vol. 33, no. 4, pp. 849-860, 2014.

[104] H. Bay, A. Ess, T. Tuytelaars, and L. Van Gool, "Speeded-up robust features (surf)," Computer vision and image understanding, vol. 110, no. 3, pp. 346359, 2008.

[105] M. Wang, L. L. S. Ong, J. Dauwels, and H. H. Asada, "Automated tracking of cells from phase contrast images by multiple hypothesis kalman filters," in Acoustics, Speech and Signal Processing (ICASSP), 2015 IEEE International Conference on. IEEE, 2015, pp. 942-946.

[106] L. Shapiro and G. Stockman, "Computer vision. 2001," ed: Prentice Hall, 2001.

[107] R. O. Duda, P. E. Hart, and D. G. Stork, Pattern classification. John Wiley \& Sons, 2012.

[108] N. Otsu, "A threshold selection method from gray-level histograms," Automatica, vol. 11, no. 285-296, pp. 23-27, 1975.

[109] M. Wang, L. L. S. Ong, J. Dauwels, and H. H. Asada, "Automatic detection of endothelial cells in $3 \mathrm{~d}$ angiogenic sprouts from experimental phase contrast images," in SPIE Medical Imaging. International Society for Optics and Photonics, 2015, pp. 94132I-94 132I.

[110] S. Lior, D. Eckley, M. Tomasz, J. Josiah, G. Ilya et al., "Wndchrm-an open source utility for biological image analysis," Source Code for Biology and Medicine, vol. 3, 2008. 
[111] H. Abdi, "Partial least squares regression and projection on latent structure regression (pls regression)," Wiley Interdisciplinary Reviews: Computational Statistics, vol. 2, no. 1, pp. 97-106, 2010.

[112] M. Potente, H. Gerhardt, and P. Carmeliet, "Basic and therapeutic aspects of angiogenesis," Cell, vol. 146, no. 6, pp. 873-887, 2011.

[113] A. Schuermann, C. S. Helker, and W. Herzog, "Angiogenesis in zebrafish," in Seminars in cell \& developmental biology, vol. 31. Elsevier, 2014, pp. 106-114.

[114] B. Vailhé, D. Vittet, J.-J. Feige et al., "In vitro models of vasculogenesis and angiogenesis," Laboratory investigation, vol. 81, no. 4, pp. 439-452, 2001.

[115] L. Tranqui and P. Tracqui, "Mechanical signalling and angiogenesis. the integration of cell-extracellular matrix couplings," Comptes Rendus de l'Académie des Sciences-Series III-Sciences de la Vie, vol. 323, no. 1, pp. 31-47, 2000.

[116] A. Haeger, K. Wolf, M. M. Zegers, and P. Friedl, "Collective cell migration: guidance principles and hierarchies," Trends in cell biology, vol. 25, no. 9, pp. 556-566, 2015.

[117] M. A. Chaplain, "Mathematical modelling of angiogenesis," Journal of neurooncology, vol. 50, no. 1-2, pp. 37-51, 2000.

[118] H. Parsa, R. Upadhyay, and S. K. Sia, "Uncovering the behaviors of individual cells within a multicellular microvascular community," Proceedings of the National Academy of Sciences, vol. 108, no. 12, pp. 5133-5138, 2011.

[119] I. Arnaoutova and H. K. Kleinman, "In vitro angiogenesis: endothelial cell tube formation on gelled basement membrane extract," Nature protocols, vol. 5, no. 4, pp. 628-635, 2010.

[120] J. Angulo and S. Matou, "Automatic quantification of in vitro endothelial cell networks using mathematical morphology," in Proceedings of the 5th IASTED 
International Conference on Visualization, Imaging, and Image Processing (VIIP'05), 2005, pp. 51-56.

[121] — "Application of mathematical morphology to the quantification of in vitro endothelial cell organization into tubular-like structures," Cellular and Molecular Biology, vol. 53, no. 2, pp. 22-35, 2007.

[122] Y.-C. Lin and P.-J. Chiang, "Quantitative analysis of vascular structures using image processing," in Computational Intelligence, Communication Systems and Networks (CICSyN), 2012 Fourth International Conference on. IEEE, 2012, pp. 278-283.

[123] F. Zhao, J. Liang, X. Chen, J. Liu, D. Chen, X. Yang, and J. Tian, "Quantitative analysis of vascular parameters for micro-ct imaging of vascular networks with multi-resolution," Medical \& biological engineering $\&$ computing, vol. 54, no. 2-3, pp. 511-524, 2016.

[124] K. Palágyi and A. Kuba, "A 3d 6-subiteration thinning algorithm for extracting medial lines," Pattern Recognition Letters, vol. 19, no. 7, pp. 613-627, 1998.

[125] T.-Q. Yan and C.-X. Zhou, "A continuous skeletonization method based on distance transform," in International Conference on Intelligent Computing. Springer, 2012, pp. 251-258.

[126] Y. Zhou and A. W. Toga, "Efficient skeletonization of volumetric objects," IEEE transactions on visualization and computer graphics, vol. 5, no. 3, pp. 196-209, 1999.

[127] M. Wan, Z. Liang, Q. Ke, L. Hong, I. Bitter, and A. Kaufman, "Automatic centerline extraction for virtual colonoscopy," IEEE transactions on medical imaging, vol. 21, no. 12, pp. 1450-1460, 2002. 
[128] M. S. Hassouna and A. A. Farag, "Robust centerline extraction framework using level sets," in 2005 IEEE Computer Society Conference on Computer Vision and Pattern Recognition (CVPR'05), vol. 1. IEEE, 2005, pp. 458-465.

[129] S. Bouix, K. Siddiqi, and A. Tannenbaum, "Flux driven automatic centerline extraction," Medical Image Analysis, vol. 9, no. 3, pp. 209-221, 2005.

[130] A. Telea and J. J. Van Wijk, "An augmented fast marching method for computing skeletons and centerlines," in Proceedings of the symposium on Data Visualisation 2002. Eurographics Association, 2002, pp. 251-ff.

[131] J. A. Sethian, "A fast marching level set method for monotonically advancing fronts," Proceedings of the National Academy of Sciences, vol. 93, no. 4, pp. 1591-1595, 1996.

[132] R. Kimmel, D. Shaked, N. Kiryati, and A. M. Bruckstein, "Skeletonization via distance maps and level sets," in Photonics for Industrial Applications. International Society for Optics and Photonics, 1995, pp. 137-148.

[133] R. Strzodka and A. Telea, "Generalized distance transforms and skeletons in graphics hardware," in Proceedings of the Sixth Joint Eurographics-IEEE TCVG conference on Visualization. Eurographics Association, 2004, pp. $221-230$.

[134] W. Gonzalez and R. E. Woods, "Eddins, digital image processing using matlab," Third New Jersey: Prentice Hall, 2004.

[135] R. M. Haralock and L. G. Shapiro, Computer and robot vision. AddisonWesley Longman Publishing Co., Inc., 1991.

[136] F. Pedregosa, G. Varoquaux, A. Gramfort, V. Michel, B. Thirion, O. Grisel, M. Blondel, P. Prettenhofer, R. Weiss, V. Dubourg et al., "Scikit-learn: Machine learning in python," The Journal of Machine Learning Research, vol. 12, pp. 2825-2830, 2011. 
[137] S. Abe, Support vector machines for pattern classification. Springer, 2010.

[138] C. Orsenigo and C. Vercellis, "Discrete support vector decision trees via tabu search," Computational statistics \& data analysis, vol. 47, no. 2, pp. 311-322, 2004 .

[139] A. Lumsdaine, L. Lee, and J. Siek, "The boost graph library: User guide and reference manual," 2002.

[140] H. W. Kuhn, "The hungarian method for the assignment problem," Naval research logistics quarterly, vol. 2, no. 1-2, pp. 83-97, 1955.

[141] J. G. Michaels and K. H. Rosen, Applications of discrete mathematics. McGraw-Hill Higher Education, 1991.

[142] R. E. Burkard, M. Dell'Amico, and S. Martello, Assignment Problems, Revised Reprint. Siam, 2009.

[143] R. del Toro, C. Prahst, T. Mathivet, G. Siegfried, J. S. Kaminker, B. Larrivee, C. Breant, A. Duarte, N. Takakura, A. Fukamizu et al., "Identification and functional analysis of endothelial tip cell-enriched genes," Blood, vol. 116, no. 19, pp. 4025-4033, 2010.

[144] I. Geudens and H. Gerhardt, "Coordinating cell behaviour during blood vessel formation," Development, vol. 138, no. 21, pp. 4569-4583, 2011.

[145] H. Gerhardt, M. Golding, M. Fruttiger, C. Ruhrberg, A. Lundkvist, A. Abramsson, M. Jeltsch, C. Mitchell, K. Alitalo, D. Shima et al., "Vegf guides angiogenic sprouting utilizing endothelial tip cell filopodia," The Journal of cell biology, vol. 161, no. 6, pp. 1163-1177, 2003.

[146] G. Cumming, F. Fidler, and D. L. Vaux, "Error bars in experimental biology," The Journal of cell biology, vol. 177, no. 1, pp. 7-11, 2007. 
[147] E. Mazor, A. Averbuch, Y. Bar-Shalom, and J. Dayan, "Interacting multiple model methods in target tracking: a survey," IEEE transactions on aerospace and electronic systems, vol. 34, no. 1, pp. 103-123, 1998.

[148] C. Kreucher, A. Hero, and K. Kastella, "Multiple model particle filtering for multitarget tracking," in the twelfth annual workshop on adaptive sensor array processing. Lexington, MA, 2004.

[149] A. Anderson and M. Chaplain, "Continuous and discrete mathematical models of tumor-induced angiogenesis," Bulletin of mathematical biology, vol. 60, no. 5, pp. 857-899, 1998. 